\title{
In situ microbubble production via flow-focusing microfluidic devices as a means to achieve large transiently-stable microbubbles for improved ultrasound image contrast and drug delivery
}

\author{
A Dissertation \\ Presented to
}

the Faculty of the School of Engineering and Applied Science

University of Virginia

\author{
In Partial Fulfillment \\ of the requirements for the Degree \\ Doctor of Philosophy (Biomedical Engineering) \\ by
}

Ali Haider Dhanaliwala

August 2014 
(c) 2014 Ali Haider Dhanaliwala 


\section{Abstract}

Ultrasound remains a mainstay of diagnostic imaging due to its portability, cost, and safety profile. Similarly, gas filled microbubbles remain the agent of choice for enhancing ultrasound contrast and therapy. Currently, microbubbles are designed to be stable in order to facilitate storage and provide an acceptable duration of contrast in vivo. This requirement to be stable reduces the acceptable range of microbubble diameters since microbubbles that are both large $(>10 \mu \mathrm{m}$ diameter $)$ and stable pose an increased risk of gas emboli formation. Unfortunately, this limitation in diameter also limits acoustic contrast as the reflected acoustic power from a microbubble scales with the square of the microbubble diameter. With access to transiently-stable microbubbles, however, large diameter microbubbles could potentially be investigated and utilized as rapid dissolution could be relied upon to prevent gas emboli formation,

Microbubble production via microfluidic devices presents an opportunity to rethink microbubble design and potentially gain access to larger diameter microbubbles and the concomitant improvements in contrast and therapy. Microfluidics enables microbubble production with devices capable of being miniaturized to vascular compatible dimensions. By integrating a microfluidic device within a catheter, microbubbles could be produced in situ, directly within the vasculature. Direct microbubble production within the vasculature could eliminate the need for highly stable microbubbles since there would be no requirement for long-term storage or multiple passes through the circulation to reach the target of interest.

By removing the stability requirement, the in situ microbubble production paradigm 
enables the development of transiently-stable microbubbles. In this dissertation, I present work towards the development of microfluidics as a means to produce transiently-stable microbubbles in order to gain access to large diameter microbubbles that would present minimal risk of gas emboli formation.

First, I present a method to reduce the footprint of microfluidic devices by simplifying tubing interconnects. In this method, a microfluidic device is directly connected to a chamber containing pressurized liquid, thus eliminating bulky interconnects. Next, I describe a model to predict microbubble production rate as a function of gas and liquid input parameters. Access to models such as this helps to guide device development and operation. Third, I present experiments towards the optimization of albumin and nitrogen for the production of transiently stable microbubbles. To investigate drug delivery, I present results characterizing the drug delivery enhancement achieved in vitro using in situ produced microbubbles. Finally, these studies culminate in the in vivo characterization of transiently-stable microfluidicproduced microbubbles directly administered from the microfluidic device into the mouse vasculature.

Overall, I observed that transiently-stable microbubbles can be fabricated with albumin and nitrogen and have a half-life of under $10 \mathrm{~s}$. Despite the short half-life, these microbubbles are capable of providing sufficient acoustic contrast and can enhance drug delivery. Furthermore, stabilization with albumin suggests the possibility of directly using plasma for microbubble stabilization. Most significantly, however, when administered in vivo transiently-stable microbubbles provide acoustic contrast without causing harm. 


\section{Approval Sheet}

This dissertation is submitted in partial fulfillment of the requirements for the degree of Doctor of Philosophy (Biomedical Engineering)

Ali Haider Dhanaliwala

This dissertation has been read and approved by the Examining Committee:

John A. Hossack PhD, Advisor, Biomedical engineering

Craig H. Meyer PhD, Committee Chair, Biomedical engineering

Scott T. Acton PhD, Electrical engineering

Alexander L. Klibanov PhD, Medicine - cardiovascular division

Richard J. Price PhD, Biomedical engieenering

F. William Mauldin PhD, Biomedical engineering

Michael Salerno MD,PhD, Medicine - cardiovascular division

Accepted for the School of Engineering and Applied Science:

James H. Aylor, Dean, School of Engineering and Applied Science August 2014 
To Soren who kept me up at night and Kaitlyn who let me sleep 


\section{Acknowledgments}

First, and foremost I would like to acknowledge the contributions of Johnny Chen, Shiying Wang, and Adam Dixon with whom much of the microfluidic work was done in collaboration. Next, I would like to thank my advisor for all his advice and insights during my training. A special thanks to Dr. Dede Haverstick and Craig Goodman for providing biologic samples and my MSTP classmates for the optimal mix of science and social. Finally, I would like to thank all my labmates and friends, with a special mention to Will, Linsey, Joe, Sam, Geoff, Kevin, and Dan, who have helped me along the way.

This work was supported in part by NIH (NHLBI) HL090700 the UVa Cardiovascular Research Center Training grant (NIH NHLBI T32HL007284), an American Heart Association fellowship, a Virginia Space Grant Consortium fellowship, and the UVa Medical Scientist

Training Program. The content is solely my responsibility does not necessarily represent the official views of the NIH, AHA, or VSGC. 


\section{Contents}

Contents vi vi vis

List of Tables . . . . . . . . . . . . . . . . . . . . . .

List of Figures . . . . . . . . . . . . . . . . . . . . xi

List of Nomenclature . . . . . . . . . . . . . . . . . . xiii

1 Introduction $\quad 1$

1.1 Clinical ultrasound . . . . . . . . . . . . . . . . . . . . . 1

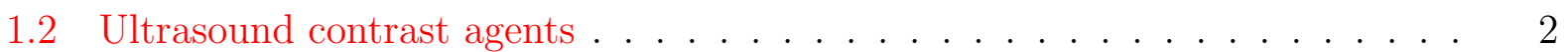

1.2 .1 Microbubbles . . . . . . . . . . . . . . . . 2

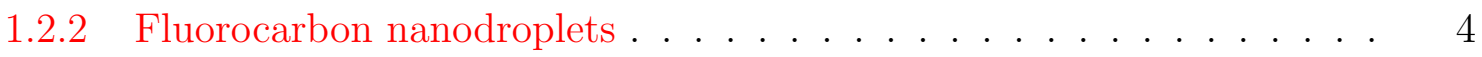

1.3 Microfluidics . . . . . . . . . . . . . . . . . . . . 5

1.3.1 Flow focusing microfluidic devices . . . . . . . . . . . . . 6

1.4 Acoustic radiation force imaging . . . . . . . . . . . . . . . . . . . . 7

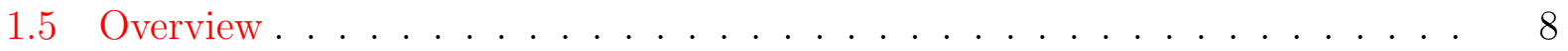

$\begin{array}{lr}\text { I Microfluidics } & 11\end{array}$

2 Liquid flooded flow-focusing microfluidic device for in situ generation of monodisperse microbubbles 12

2.1 Abstract . . . . . . . . . . . . . . . . . . . . . 12

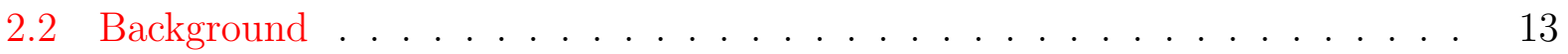

2.3 Materials and Methods . . . . . . . . . . . . . . . . . . 17

2.3.1 Microfluidic device fabrication . . . . . . . . . . . . . . . 17

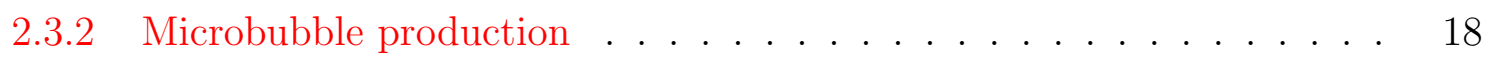

2.3.3 Acoustic imaging . . . . . . . . . . . . . . . . . . . . . . . . . . . . . . . . .

2.4 Results and discussion . . . . . . . . . . . . . . . . . . . 21

2.4.1 FFMD fabrication and shell material optimization . . . . . . . . . 21

2.4.2 Microbubble production and characterization . . . . . . . . . . . 23

2.4.3 Imaging In situ produced microbubbles . . . . . . . . . . . . . . . . . 25

2.5 Conclusion . . . . . . . . . . . . . . . . . . . . . . . 29

3 Characterization of an expanding-nozzle flow-focusing microfluidic device for the development of a model to predict production rate as a function of liquid flow rate and gas pressure 
3.1 Abstract . . . . . . . . . . . . . . . . . . . 31

3.2 Background . . . . . . . . . . . . . . . . . . . . . 32

3.2 .1 Theory . . . . . . . . . . . . . . . . . . . . . . . . 33

3.3 Material and Methods . . . . . . . . . . . . . . . . . . . 34

3.3.1 Microfluidic device fabrication . . . . . . . . . . . . . . . . 34

3.3.2 Microbubble production ................... . 34

3.3 .3 Filter characterization . . . . . . . . . . . . . . . . 35

3.4 Results and Discussion . . . . . . . . . . . . . . . . . . . . . . . . 36

3.4 .1 Device fabrication . . . . . . . . . . . . . . . . . . . . 36

3.4.2 Flow conditions and experimental results . . . . . . . . . . 37

3.4 .3 Microbubble Generation Regimes . . . . . . . . . . . . . . . . . 37

3.4.4 Microbubble generation modes . . . . . . . . . . . . . . . . . . . . . . 39

3.4.5 Microbubble production rate . . . . . . . . . . . . . . . . 40

3.4 .6 Limitations . . . . . . . . . . . . . . . . . . . . . . . . 42

3.5 Conclusion . . . . . . . . . . . . . . . . . . . . . . . 44

4 Investigation of albumin stabilization for the production of transientlystable microbubbles from a flow-focusing microfluidic device

4.1 Abstract . . . . . . . . . . . . . . . . . . . 45

4.2 Background . . . . . . . . . . . . . . . . . . . . . 46

4.3 Materials and Methods . . . . . . . . . . . . . . . . . . 47

4.3 .1 FFMD fabrication . . . . . . . . . . . . . . . . 47

4.3 .2 Microbubble fabrication . . . . . . . . . . . . . . . . . . 47

4.3 .3 Microbubble characterization . . . . . . . . . . . . . . . 48

4.3 .4 Plasma microbubbles . . . . . . . . . . . . . . . . . . . . . . 48

4.4 Results . . . . . . . . . . . . . . . . . . . . . . . . . . 49

4.4.1 Albumin microbubble production and coalesence . . . . . . . . . 49

4.4 .2 BSA microbubble stability . . . . . . . . . . . . . . . 50

4.4 .3 Bovine plasma microbubbles . . . . . . . . . . . . . . . . 52

4.5 Discussion . . . . . . . . . . . . . . . . . . . 53

4.5.1 Microbubble coalescence and stability . . . . . . . . . . . 53

4.5 .2 Plasma microbubbles . . . . . . . . . . . . . . . . . . . . . . 53

4.5 .3 Limitations . . . . . . . . . . . . . . . . . . . . 54

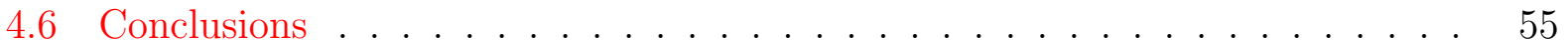

5 Microfluidic-produced microbubbles for enhanced drug delivery in vitro $\mathbf{5 6}$

5.1 Abstract . . . . . . . . . . . . . . . . . 56

5.2 Background . . . . . . . . . . . . . . . . . . 57

5.3 Materials and Methods . . . . . . . . . . . . . . . . . . 58

$5.3 .1 \quad$ FFMD fabrication . . . . . . . . . . . . . . 58

5.3 .2 Microbubble characterization . . . . . . . . . . . . . . . . 59

5.3 .3 Cell culture and calcein delivery . . . . . . . . . . . . . . . . . . 59

5.3 .4 Cell image analysis . . . . . . . . . . . . . . . . . . . . 61

5.4 Results . . . . . . . . . . . . . . . . . . . . . . . 62

5.4 Microbubble characterization . . . . . . . . . . 62 
5.4 .2 Microbubble stability . . . . . . . . . . . . . . . 63

5.4 .3 Drug Delivery - pressure . . . . . . . . . . . . . 63

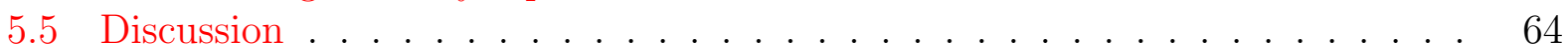

5.5.1 FFMD and microbubble characterization . . . . . . . . . . 64

5.5.2 Drug delivery and pressure . . . . . . . . . . . . . 66

5.6 Conclusions . . . . . . . . . . . . . . . . . 67

6 In vivo imaging of microfluidic-produced microbubbles $\quad 68$

6.1 Abstract . . . . . . . . . . . . . . . . . . . 68

6.2 Background . . . . . . . . . . . . . . . . . . . . 69

6.3 Materials and Methods . . . . . . . . . . . . . . . . . 69

6.3.1 Microfluidic production of microbubbles . . . . . . . . . . . 69

6.3 .2 In vivo imaging . . . . . . . . . . . . . . . . . . . 71

6.3.3 Microbubble oscillation model . . . . . . . . . . . . . . . . . 72

6.3.4 Microbubble dissolution model . . . . . . . . . . . . . 73

6.4 Results . . . . . . . . . . . . . . . . . . . . . . 74

6.4.1 In vivo imaging . . . . . . . . . . . . . . . . . . . . . . . . . . . . . . . . . . . . 74

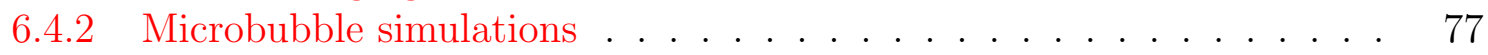

6.5 Discussion . . . . . . . . . . . . . . . . . . . . 80

6.5.1 In vivo imaging . . . . . . . . . . . . . . . . . . . . 80

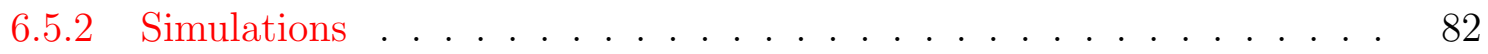

6.5.3 Limitations . . . . . . . . . . . . . . . . . 85

6.6 Conclusion . . . . . . . . . . . . . . . . . 86

\section{$\begin{array}{llr}\text { II } & \text { ARFI and aaRBCs } & 87\end{array}$}

7 Assessing and improving acoustic radiation force image quality using a 1.5D transducer design

7.1 Abstract . . . . . . . . . . . . . . . . . . 88

7.2 Background . . . . . . . . . . . . . . . . . . . . . 89

7.3 Theory and Methods . . . . . . . . . . . . . . . . . . 90

7.3.1 Derivation of ARFI image SNR upper bound . . . . . . . . . . . . . . 90

7.3.2 Numerical simulation framework . . . . . . . . . . . . . . . . . 94

7.3.3 1.5D ARFI transducer design and comparison . . . . . . . . . . . . 95

7.4 Results and discussion . . . . . . . . . . . . . . . . . . . . . 98

7.5 Conclusion . . . . . . . . . . . . . . . . . . . . . . . . 101

8 Acoustically active red blood cells as an acoustically triggered drug deliv$\begin{array}{ll}\text { ery vehicle } & 103\end{array}$

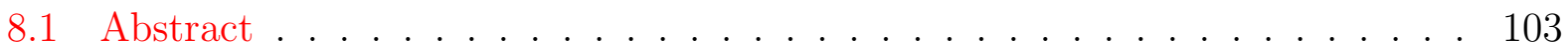

8.2 Background . . . . . . . . . . . . . . . . . . 104

8.3 Materials and Methods . . . . . . . . . . . . . . . . . . 106

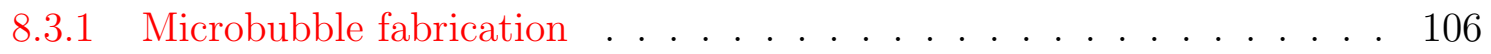

8.3.2 Microbubble precursor fabrication . . . . . . . . . . . . . 106 
8.3.3 Acoustically-active red blood cell production . . . . . . . . . . . . . 107

8.3 .4 Microbubble Survival . . . . . . . . . . . . . . . . . . . 109

8.3.5 Acoustic conversion of microbubble precursors . . . . . . . . . . . . 109

8.3.6 Stability of microbubble precursors . . . . . . . . . . . . . 110

8.3.7 Drug loading and triggered release . . . . . . . . . . . . . 110

8.4 Results and Discussion . . . . . . . . . . . . . . . . . . 111

8.4.1 Microbubble precursor characterization . . . . . . . . . . . 111

8.4.2 Microbubble stability . . . . . . . . . . . . . . . . 115

8.4.3 Red blood cell ghosts - Microbubble loading . . . . . . . . . . . . . 115

8.4.4 Red blood cell ghosts - Microbubble precursor loading . . . . . . . . . 118

8.4.5 Triggered Release . . . . . . . . . . . . . . . . . . 119

8.5 Conclusion . . . . . . . . . . . . . . . . . . . . 121

9 Conclusions and future directions 122

9.1 Final conclusions . . . . . . . . . . . . . . . . . . . . . . . 122

9.2 Limitations . . . . . . . . . . . . . . . . . . . . 126

9.3 Future directions . . . . . . . . . . . . . . . . . 127

$\begin{array}{lr}\text { Appendices } & 129\end{array}$

$\begin{array}{ll}\text { A Publications } & 130\end{array}$

B Numerical evaluation of the scattering cross section of a microbubble 132

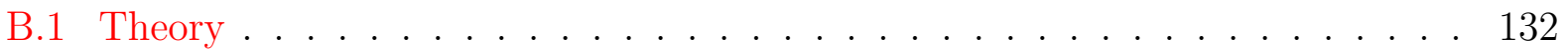

B.2 Results and Discussion . . . . . . . . . . . . . . . . 133

$\begin{array}{ll}\text { C Fluid dynamic relationships } & 135\end{array}$

D Dissolution model of fluorocarbon-nitrogen microbubbles $\quad 137$

D.1 Theory . . . . . . . . . . . . . . . . . . . 137

D.2 Results and Discussion . . . . . . . . . . . . . . . . . . 140

E Mass spectrometry of Zonyl (FS-300) 142

F $\quad$ Red blood cell ghost particle loading protocol $\quad 145$

F.1 Microbubble precursor fabrication . . . . . . . . . . . . . . . 146

F.2 Red blood cell ghost loading . . . . . . . . . . . . . . . . . . . 147

$\begin{array}{ll}\text { Bibliography } & 151\end{array}$ 


\section{List of Tables}

1.1 Acoustic impedance of common tissue, liquids, and gases at $37^{\circ} \mathrm{C}$ and $101 \mathrm{kPa} \quad 3$

2.1 Microbubble production capabilities for an iFFMD and eFFMD . . . . . 25

3.1 Microfluidic device dimensions . . . . . . . . . . . . . . . . . . 35

3.2 Flow conditions and experimental results when operating in the stable regime

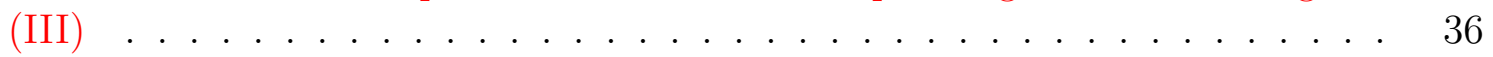

3.3 Production regimes at fixed gas pressure . . . . . . . . . . . . . . 39

4.1 Microbubble half-life . . . . . . . . . . . . . . . . . 51

6.1 Microbubble oscillation simulation parameters . . . . . . . . . . . . 73

6.2 Microbubble dissolution simulation parameters . . . . . . . . . . . . . . 74

7.1 Simulation parameters for the conventional 1D array and 1.5D ARFI array . 97

7.2 Tissue displacement width, tracking beam width and the ratio of displacement width to tracking width for the 1.5D ARFI and the conventional 1D array . 99

7.3 CNR and SNR values of the simulated lesion when imaged with the conventional $1 \mathrm{D}$ and the $1.5 \mathrm{D}$ ARFI array . . . . . . . . . . . . . . . . 100

8.1 Boiling and melting point of selected common fluorocarbons . . . . . . . . 111

8.2 B-mode transmit power required to convert microbubble precursors into gas microbubbles ........................ 115

D.1 Fluorocarbon microbubble dissolution parameters . . . . . . . . . . . 138

D.2 Octafluoropropane coefficients . . . . . . . . . . . . . . . . 139

D.3 Dissolution times . . . . . . . . . . . . . . . . . . . . . . . . . . . 140

E.1 Potential mass to charge ratios for Zonyl polymers of different lengths. . . . 143 


\section{List of Figures}

1.1 Agitation versus microfluidic methods for microbubble production . . . . . . 6

2.1 Example microfluidic interconnects . . . . . . . . . . . . . . . 16

2.2 Flooded FFMD schematic . . . . . . . . . . . . . . . . . . . . . . . 19

2.3 Externally and internally mounted flooded FFMDs . . . . . . . . . . . . 22

2.4 Microbubble production regimes of a flooded FFMD . . . . . . . . . . . . . . 24

2.5 Relationship between microbubble diameter and production as a function of gas pressure and liquid flow rate . . . . . . . . . . . . . . 26

2.6 Ultrasound images of PEG40St microbubbles produced in situ via an eFFMD and imaged in real time . . . . . . . . . . . . . . . . . . 28

2.7 IVUS images of in situ produced microbubbles . . . . . . . . . . . . . . . . 29

3.1 Expanding nozzle flow-focusing microfluidic device schematic . . . . . . . . . 34

3.2 Scanning electron microscopy of FFMD SU-8 mold . . . . . . . . . . . . . 36

3.3 Microbubble production regimes . . . . . . . . . . . . . . 38

3.4 Microbubble generation at the gas cone tip . . . . . . . . . . . . . . . 40

3.5 Predicted microbubble production versus measured results . . . . . . . . 43

4.1 Effect of BSA, dextrose and GPS concentration on microbubble coalescence . 50

4.2 Acoustic determination of microbubble half-life . . . . . . . . . . . . 51

4.3 Plasma-stabilized microbubbles . . . . . . . . . . . . . . . . . 52

5.1 Schematic of flow chamber for drug delivery . . . . . . . . . . . . . 60

5.2 Calcein incorporated into the liquid phase of the FFMD . . . . . . . . . . 62

5.3 Size distribution of the FFMD-produced microbubbles as a function of time . 64

5.4 Fluorescence microscopy images of rat aortic smooth muscle cells following ultrasound-mediated calcein delivery . . . . . . . . . . . . . 65

5.5 Calcein delivery efficiency and percent cell death versus peak negative pressure 66

6.1 Schematic of the flow-focusing microfluidic device . . . . . . . . . . . 70

6.2 Microfluidic-produced microbubble characteristics . . . . . . . . . . . 75

6.3 Pre-contrast and post-contrast images of the right and left ventricles . . . . 76

6.4 Size distribution of agitation-produced microbubbles . . . . . . . . . . . 77

6.5 Rise time, decay time, and duration of image contrast in the right ventricle . 78

6.6 Correlation between maximum image intensity and microbubble diameter, production rate or the product of microbubble volume and production rate . 78 
6.7 Simulated pressure from a microbubble as a function diameter and frequency 79

6.8 Simulated microbubble lifetime . . . . . . . . . . . . . . . . . . . 80

6.9 Representative B-mode images . . . . . . . . . . . . . . . . . . 85

7.1 Block diagram of the numerical simulation framework . . . . . . . . . . . 96

7.2 Pushing and tracking array geometry for the proposed 1.5D ARFI imaging transducer . . . . . . . . . . . . . . . . . . . . . . 97

$7.3 S N R_{A R F I}$ as a function of peak displacement . . . . . . . . . . . . . 100

7.4 Simulated image of a compliant lesion embedded in stiffer surrounding tissue imaged with the 1D and 1.5D array . . . . . . . . . . . . . 101

8.1 General red blood cell loading procedure . . . . . . . . . . . . . . 108

8.2 Diameter distribution of microbubble precursors . . . . . . . . . . . . 113

8.3 Microbubble precursor stability . . . . . . . . . . . . . . . . 114

8.4 Acoustic conversion of mircrobubble precursors . . . . . . . . . . . . . 115

8.5 Microbubble loss as a function of centrifugation speed . . . . . . . . . . . 116

8.6 The four types of aaRBCs . . . . . . . . . . . . . . . . 117

8.7 Diameter distribution of red blood cells pre- and post- lysis . . . . . . . . . 118

8.8 Red blood cells loaded with polystyrene beads . . . . . . . . . . . . . . . . 119

8.9 Calcein release from microbubble loaded red blood cells . . . . . . . . . . . . 120

9.1 Schematic of the proposed multi-function IVUS catheter . . . . . . . . . . 124

B.1 Effect of frequency and microbubble diameter on scattering cross section . . 134

D.1 Dissolution of a fluorocarbon-nitrogen microbubble . . . . . . . . . . . . 141

E.1 Mass spectroscopy of Zonyl . . . . . . . . . . . . . . . . . . . . . . . . 144

F.1 Syringe holder for maintaining pressure . . . . . . . . . . . . . . . . 148

F.2 Brightfield image of control red blood cell ghosts . . . . . . . . . . . . . . 150 


\title{
List of Nomenclature
}

\section{Mathematical Symbols}

\author{
Symbol Description \\ A Maximum scatterer displacement \\ $\alpha \quad$ Acoustic attenuation in medium \\ $B \quad$ Fractional bandwidth \\ $\beta_{b} \quad$ Slope of $\tau_{b}$ fit \\ $\beta_{w} \quad$ Slope of $\tau_{w}$ fit \\ $c \quad$ Speed of sound \\ $\mathrm{Ca} \quad$ Capillary number \\ CNR Contrast to noise ratio \\ $D_{F} \quad$ Octafluoropropane diffusion constant \\ $D_{N} \quad$ Nitrogen diffusion constant \\ $D_{x} \quad$ Width of tissue displacement in the lateral dimension \\ $D_{y} \quad$ Width of tissue displacement in elevation dimension \\ $\delta_{\text {damp }} \quad$ Damping constant \\ $\delta_{\text {radiation }}$ Radiation loss \\ $\delta_{\text {thermal }} \quad$ Thermal loss \\ $\delta_{\text {viscosity }}$ Viscous loss \\ $D_{h} \quad$ Hydraulic diameter
}

Continued on next page 


\section{Symbol Description}

\begin{tabular}{|c|c|}
\hline$d_{j}$ & Diameter of the gas jet at the microfluidic nozzle \\
\hline$E_{\text {reflection }}$ & Reflected energy \\
\hline$\eta_{L}$ & Viscosity of water \\
\hline$F$ & Acoustic radiation force \\
\hline$f_{c}$ & Insonation pulse frequency \\
\hline$f_{b}$ & Microbubble production rate \\
\hline$f_{R}$ & Microbubble resonance frequency \\
\hline$g$ & Gravitational acceleration \\
\hline$\gamma$ & Ratio of specific heat \\
\hline$h$ & Microfluidic channel height \\
\hline$I$ & Acoustic beam intensity \\
\hline$\kappa$ & Polytropic exponent \\
\hline$l$ & Characteristic length \\
\hline$L_{F}$ & Octafluoropropane Ostwald coefficient \\
\hline$L_{N}$ & Nitrogen Ostwald coefficient \\
\hline$L_{x}$ & Ratio of displacement width to tracking beam width in the lateral dimension \\
\hline$L_{y}$ & Ratio of displacement width to tracking beam width in the elevation dimension \\
\hline$\mu_{M B}$ & Mean microbubble diameter \\
\hline$\mu$ & Dynamic viscosity \\
\hline$\mu_{d}$ & Mean of estimated acoustic radiation force impulse imaging displacement \\
\hline$\mu_{i}$ & Mean displacement inside lesion \\
\hline$\mu_{o}$ & Mean displacement outside lesion \\
\hline$\mu_{w}$ & Mean pixel intensity within the lumen with microbubbles \\
\hline$\mu_{w o}$ & Mean pixel intensity within the lumen without microbubbles \\
\hline$M W_{Z}$ & Zonyl molecular weight \\
\hline
\end{tabular}




\section{Symbol Description}

\begin{tabular}{ll}
$N_{A}$ & Avogadro's number \\
$\nu$ & Poisson's ratio \\
$\omega$ & Microbubble breakup frequency \\
$P$ & Input gas pressure \\
$p_{a m b}$ & Ambient pressure \\
$p_{\text {blood }}$ & Overpressure in blood \\
$p_{\text {drive }}$ & External driving pressure \\
$p_{\text {int }}$ & Internal microbubble pressure \\
$P_{M B}$ & Pressure wave from an oscillating microbubble \\
$P I$ & Polydispersity index \\
$Q_{l}$ & Liquid flow rate \\
$R$ & Radius \\
$R_{0}$ & Initial microbubble radius \\
$r$ & Distance from microbubble \\
$r_{d r o p}$ & Radius of microbubble precursor \\
$R e$ & Reynolds number \\
$\rho_{\text {cor }}$ & Echo correlation \\
$\rho_{d r o p}$ & Microbubble precursor density \\
$\rho_{g}$ & Gas density \\
$\rho_{L}$ & Density of water \\
$S A_{Z}$ & Surface area of single Zonyl molecule \\
$S_{Z}$ & Concentration of stock Zonyl \\
$S C_{d r o p}$ & Settling velocity of microbubble precursor \\
$\sigma$ & Surface tension \\
\hline
\end{tabular}

Continued on next page 


\section{Symbol Description}

$\sigma_{d} \quad$ Standard deviation (or "jitter") of estimated acoustic radiation force impulse imaging displacement

$\hat{\sigma_{d}} \quad$ Minimum achievable jitter of displacement estimator

$\sigma_{M B} \quad$ Standard deviation of microbubble diameter

$\sigma_{o} \quad$ Standard deviation of displacement outside lesion

$\sigma_{w o} \quad$ Standard deviation of pixel intensity within the lumen without microbubbles

$\sigma_{X C} \quad$ Scattering cross section

$S N R_{A R F I}$ Acoustic radiation force impulse imaging signal to noise

$S N R_{E} \quad$ Electronic signal to noise of ultrasound system

T Motion estimator window length

$T_{x} \quad$ Width of tracking beam in the lateral dimension

$T_{y} \quad$ Width of tracking beam in the elevation dimension

$\tau \quad$ Time gas thread stays in microfluidic nozzle

$\tau_{b} \quad$ Bubbling time

$\tau_{s} \quad$ Shear stress

$\tau_{w} \quad$ Waiting time

$\theta_{b} \quad$ Microfluidic nozzle flare angle

$\theta_{l} \quad$ Microfluidic liquid channel flare angle

$u \quad$ Mean flow velocity

$V_{g} \quad$ Volume of gas

$V_{m} \quad$ Molar volume

$V_{Z} \quad$ Zonyl volume

$\varepsilon_{b} \quad$ Offset of $\tau_{b}$ fit

$\varepsilon_{w} \quad$ Offset of $\tau_{w}$ fit

$w \quad$ Microfluidic nozzle width

Continued on next page 


\section{Symbol Description}

$W_{x} \quad$ Width of pushing beam in the lateral dimension

$W_{y} \quad$ Width of tracking beam in the elevation dimension

$w_{g} \quad$ Microfluidic gas channel width

$w_{l} \quad$ Microfluidic liquid channel width

We Weber number

$X \quad$ Gravitational multiplier (i.e. RCF)

Z Acoustic impedance 


\title{
Acronyms
}

\author{
Acronym Description \\ aaRBC Acoustically active red blood cell \\ ADV Acoustic droplet vaporization \\ APTES (3-Aminopropyl)triethoxysilane \\ ARFI Acoustic radiation force impulse \\ BBB Blood brain barrier \\ BSA Bovine serum albumin \\ $\mathrm{C}_{3} \mathrm{~F}_{8} \quad$ Octafluoropropane \\ $\mathrm{C}_{4} \mathrm{~F}_{10} \quad$ Decafluorobutane \\ CEHDA Coaxial electrohydrodynamic atomisation \\ CPS Contrast pulse sequence \\ CT Computed tomography \\ DI Deionized water \\ DiI $\quad 1,1^{\prime}$-Dioctadecyl-3,3, $3^{\prime}, 3^{\prime}$ tetramethylindocarbocyanine perchlorate \\ DLS Dynamic light scattering \\ DPPC 1,2-dipalmitoyl-sn-glycero-3-phosphocholine \\ DSPC 1,2-distearoyl-sn-glycero-3-phosphocholine \\ eFFMD Externally mounted flooded flow-focusing microfluidic device \\ FDG Fluorodeoxyglucose \\ FEM Finite element model \\ FFMD Flow-focusing microfluidic device \\ FWHM $\quad$ Full width half maximum \\ GP Glycerol, propylene glycol \\ GPS Glycerol, propylene glycol, isotonic saline solution \\ GPS+ Glycerol, propylene glycol, PBS + calcium + magnesium solution \\ Continued on next page
}




\begin{tabular}{ll} 
Acronym & Description \\
HSA & Human serum albumin \\
iFFMD & internally mounted flooded flow-focusing microfluidic device \\
IVUS & Intravascular ultrasound \\
LV & Left ventricle \\
MB & Microbubbles \\
MI & Mechanical index \\
MRI & Magnetic resonance imaging \\
PBS & Phosphate buffered saline \\
PBS $+/+$ & Phosphate buffered saline + calcium + magnesium \\
PDMS & poly-dimethyl-siloxane \\
PEG40St & Polyethylene-glycol-40-stearate \\
PET & Positron emission tomography \\
PI & Propidium iodide \\
PNP & Peak negative pressure \\
pRBC & Packed red blood cells \\
RTEE & Polytetrafluoroethylene \\
RES & Rediculoendothelial system \\
RV & Ultrasound \\
\hline
\end{tabular}




\section{Chapter 1}

\section{Introduction}

\subsection{Clinical ultrasound}

Ultrasound as a medical imaging modality dates back to the early 1940s when advances in SONAR and acoustic flaw detectors converged to provide physicians with a compact technology for noninvasively probing the human body with sound [1]. From those early beginnings, clinical ultrasound has rapidly evolved into a sophisticated imaging modality capable of using sound to provide anatomic, functional, and molecular information of the human body [2, 3, 4]. Not surprisingly, given its portability, cost, and versatility, ultrasound is the most common medical imaging modality and the only imaging modality to be sent into space [5].

As its name suggests, ultrasound uses high frequency sound waves $(>1 \mathrm{MHz})$ to noninvasively interrogate the body. In the most general implementation, a transducer capable of producing megahertz acoustic waves is used to emit sound into the body. Those sound waves reflect off boundaries within the body, and the reflections are recorded by the same transducer used to emit the waves. The recorded signal is then processed in hardware and software to produce an amplitude line (A-line). The more energy reflected by a given boundary, the larger the amplitude in the resulting A-line. Furthermore, since the speed of sound is approximately 
equal throughout the body, the time delay of the signal can be used to determine the depth of the boundary. By repeating this process, adjacent A-lines can be acquired and combined to produce a 2-D brightness (B-mode) image.

Ultrasound, unlike other medical imaging modalities, also has a long history of therapeutic applications $[6,7]$. Although early therapeutic implementations were anecdotal, therapeutic ultrasound has seen a resurgence in interest due to exciting results with neurostimulation [8], histotripsy [9], and MR-guided focused ultrasound [10]. In addition, ultrasound contrast agents uniquely enable enhancements in both imaging and therapy, further increasing the functional repertoire of ultrasound.

\section{$1.2 \quad$ Ultrasound contrast agents}

Contrast agents are, in general, molecules or particles that are capable of selectively increasing the signal to noise ratio for a given imaging modality. X-ray imaging, including fluoroscopy and computed tomography (CT), uses iodinated or barium compounds; positron emission tomography (PET) uses fluorodeoxyglucose (FDG) radiotracers, and magnetic resonance imaging (MRI) uses gadolinium compounds. In ultrasound, contrast is derived from differences in acoustic impedance on either side of a boundary — larger differences result in increased reflected energy and a brighter pixel in the final image. Among the landscape of the human body (Table 1.1), air-water interfaces provide one of the largest mismatches in acoustic impedance and, as such, are highly reflective of ultrasound.

\subsubsection{Microbubbles}

First reported by Gramiak and Shah who observed enhanced acoustic contrast after injecting agitated saline into the aortic root [13], micrometer-sized air bubbles were soon after identified as the source of this enhancement [14]. Air bubbles are ideal ultrasound contrast agents as their

high compressibility and low density make them strong acoustic reflectors in water. Initially 
Table 1.1: Acoustic impedance of common tissue, liquids, and gases at $37^{\circ} \mathrm{C}$ and $101 \mathrm{kPa}$

\begin{tabular}{cc}
\hline Material & Impedance $\left(\mathrm{MPa} \mathrm{s} \mathrm{m}^{-1}\right)$ \\
\hline \hline Bone & $6.36[11]$ \\
Blood & $1.68[11]$ \\
Muscle & $1.65[11]$ \\
Water & $1.51[12]$ \\
Fat & $1.33[11]$ \\
Decafluorobutane $\left(\mathrm{C}_{4} \mathrm{~F}_{10}\right)$ & $998 \times 10^{-6}[12]$ \\
Octafluoropropane $\left(\mathrm{C}_{3} \mathrm{~F}_{8}\right)$ & $892 \times 10^{-6}[12]$ \\
Nitrogen $\left(\mathrm{N}_{2}\right)$ & $395 \times 10^{-6}[12]$ \\
\hline
\end{tabular}

microbubbles were produced by simply agitating saline or indocyanine green (ICG) [15]. To increase the lifetime of microbubbles, shell materials, such as proteins, polymers, and lipids, were introduced to stabilize the microbubbles [16]. It was next realized that the replacement of air with low solubility gases such as fluorocarbons could further increase microbubble lifetime [17]. Studies investigating the clearance of microbubbles discovered that a brush border and neutral charge help avoid microbubble clearance by the reticuloendothelial system (RES) $[18,19]$, and that microbubble diameter must be less than $10 \mu \mathrm{m}$ to avoid filtration during passage through the lungs [20, 21]. Since large long-lived microbubbles have the potential to become gas emboli and cause ischemia in the microvasculature, this size limitation became even more critical as microbubble lifetime increased.

Although microbubbles were first utilized to detect wall motion abnormalities in the heart [22], advances in microbubbles, ultrasound, and image detection have led to microbubble applications throughout the body [23]. In addition to anatomic enhancements, microbubbles enable the measurement of functional quantities such as tissue blood perfusion [24] and the ability to detect the presence of molecular markers [25, 26, 27]. The development of phase-change droplets may enable microbubbles to move from being a strictly vascular-only contrast agent to an extravascular agent [28].

One disadvantage of requiring a small diameter, however, is the reduction in scattering cross section. The scattering cross section relates the power scattered by an object to the incident intensity of the wave. This ratio depends on the incident frequency of the wave, the 
radius of the object, and the differences in compressibility and density between the object and the surrounding medium. In general, particles with larger scattering cross sections will reflect more acoustic energy as described in appendix B. Consequently, the utilization of large microbubbles ( $>10 \mu \mathrm{m}$ diameter), aside from any emboli risk, should produce an improvement in image contrast as compared to smaller microbubbles [29].

Finally, in the same way that ultrasound alone can be used for both imaging and therapeutics, microbubbles can also be used both imaging and therapeutics. Microbubbles can enhance cell uptake of drugs and genes through a process termed sonoporation [30]. Furthermore, by attaching a drug or gene and a targeting ligand to the microbubble shell, microbubbles can be used to specifically target delivery to a tissue or organ [31, 32, 33]. Microbubbles have also been shown to potentiate blood brain barrier (BBB) opening, [34], thrombolysis [35], and thermal ablation [36]. Again, similar to acoustic contrast, evidence suggests that larger diameter microbubbles provide an enhanced therapeutic response in comparison to smaller diameter microbubbles [37, 38]. Consequently, if microbubbles can be fabricated to be both large and safe, then it may be possible enhance image contrast and therapeutic delivery over current achievable levels.

\subsubsection{Fluorocarbon nanodroplets}

First investigated in the 1960s as a blood substitute [39], liquid fluorocarbon nanodroplets have recently seen a resurgence in interest [40, 41, 42, 43, 44]. Unlike microbubbles that are composed of low boiling point fluorocarbons such as octafluoropropane $\left(\mathrm{C}_{3} \mathrm{~F}_{8}\right)$ or decafluorobutane $\left(\mathrm{C}_{4} \mathrm{~F}_{10}\right)$, fluorocarbon nanodroplets are typically composed of higher boiling point fluorocarbons such as dodecafluoropentane $\left(\mathrm{C}_{5} \mathrm{~F}_{12}\right)$, n-perfluorohexane $\left(\mathrm{C}_{6} \mathrm{~F}_{14}\right)$ or perfluoro-15-crown-5-ether $\left(\mathrm{C}_{15} \mathrm{~F}_{30} \mathrm{O}_{5}\right)$. Although the nanodroplets are not as compressible as microbubbles, the large difference in density and speed of sound between the fluorocarbon and water result in an acoustic reflection, especially at high frequencies [45]. By utilizing the shorter chain fluorocarbons, the droplets can be vaporized from a liquid to a gas, a 
process termed acoustic droplet vaporization (ADV) [40]. With ADV, nanometer sized liquid fluorocarbon droplets can be converted with ultrasound, specifically in space and time, into gas microbubbles. These gas microbubbles subsequently provide the same benefits of standard microbubbles as described previously. By packaging microbubbles as nanodroplets, it is possible to deliver microbubbles to locations previously unreachable. For example, small nanodroplets can be converted into large gas bubbles for gas emboli-therapy within the small vessels of a tumor [40]. Alternatively, molecular targeted nanodroplets can be allowed to extravasate from the vasculature into tissue, providing a means to image molecular targets not otherwise on the vessel wall [43].

\subsection{Microfluidics}

Currently, the most common method of microbubble fabrication involves high-shear mixing, via either mechanical agitation or sonication, of a liquid phase containing a stabilizing shell material and a gas phase [17, 46]. Agitation methods are preferred as they can quickly produce large quantities of microbubbles $\left(\approx 10^{9}\right.$ microbubbles / batch in under

1 minute). Microbubble production via agitation methods is a stochastic process. As a result, while experimental parameters can be varied to achieve a specific mean microbubble diameter, agitation methods typically produce microbubble populations with large diameter variations [46]. More significantly, however, agitation methods require bulky equipment that limits production to the benchtop.

In an attempt to remedy some of the shortcomings of microbubble production via agitation methods, microfluidic methods have been investigated [47, 48, 49, 50, 51, 52]. In general, microfluidic methods direct gas and liquid through micrometer-sized channels in order to produce shear forces that will cause microbubble generation. Unlike agitation methods, microfluidic production of microbubbles can be deterministic (Fig. 1.1). As a result, for a given set of parameters (e.g. gas and liquid flow rate, device geometry), the 


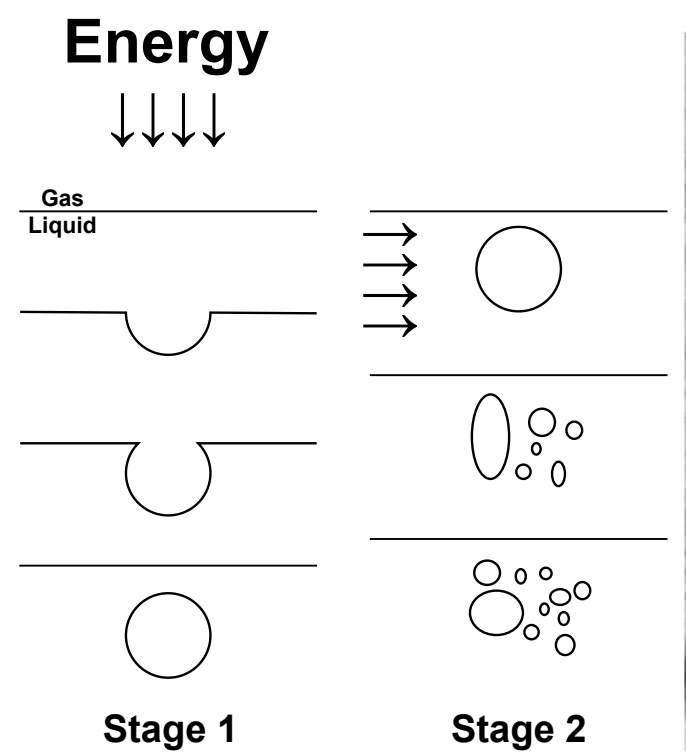

(a)

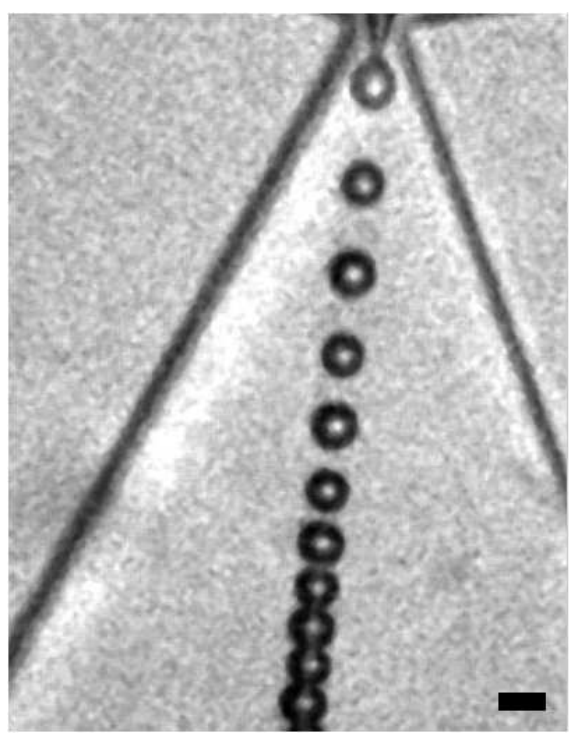

(b)

Figure 1.1: (a) Mechanical agitation results in polydisperse microbubble populations due to the stochastic nature of microbubble formation. Microbubbles are formed in two stages. In stage 1, energy causes bubble formation at the gas/liquid interface. In stage 2, energy causes the bubbles from stage 1 to break into smaller microbubbles. During both stages, surfactants in the liquid phase stabilize the microbubbles. (b) With microfluidic devices, microbubble formation is deterministic and dependent on several factors including device geometry, liquid flow rate and gas pressure. Microbubbles are formed serially and, under ideal conditions, each microbubble is identical to the previous microbubble. Scale bar $=10 \mu \mathrm{m}$.

volume of the gas bubbles is consistent after each pinch off, resulting in the production of a monodisperse population of microbubbles. This characteristic also allows microbubble diameter and production to be adjusted in real-time. Once the parameter space is known, adjusting the gas and liquid flow rate results in predictable changes in microbubble diameter and production rate [51].

\subsubsection{Flow focusing microfluidic devices}

Several microfluidic device designs for microbubble production exist including: t-junction [53], v-junction [54], co-flow [47], coaxial electrohydrodynamic atomisation (CEHDA) [55], and flow-focusing microfluidic devices (FFMD) [52, 56, 48, 49, 51, 50]. While all these designs are capable of producing monodisperse distributions of microbubbles, FFMDs have found 
preference as they are produced by casting poly-dimethyl-siloxane (PDMS), a biocompatible

synthetic polymer, onto molds fabricated using conventional microphotolithography [57, 58]. Photolithography allows intricate channel patterns to be produced at micrometer scales in a simple, low-cost, and time efficient fabrication process. FFMDs can be further categorized depending on the geometry of the outlet channel. In the expanding nozzle design, a velocity gradient develops as the gas and liquid phase pass through the narrow nozzle and into an expanding outlet [59]. As a result, microbubble pinch-off occurs at lower flow rates, reducing waste.

Similar to agitation methods, microfluidic devices for microbubble production have, to date, been fabricated for operation on a benchtop. However, unlike agitation methods, microfluidic devices have the potential to be miniaturized to vascularly compatible dimensions. With a small form factor, microfluidic devices could be coupled with a catheter, thus enabling microbubble production directly within the vasculature. By producing microbubbles in situ, loss during microbubble administration and systemic circulation could be reduced or eliminated. Furthermore, as the microbubbles would not need to be stored, transiently-stable microbubbles that utilize highly soluble gases and/or lower stability shells, could be explored. Importantly, access to transient-stable microbubbles may relax the necessity for small diameter $(<10 \mu \mathrm{m})$ microbubbles, since gas dissolution can be relied upon to prevent emboli formation. As a result, large diameter microbubbles with their concomitant improvements in acoustic contrast and drug delivery could be explored.

\subsection{Acoustic radiation force imaging}

Acoustic radiation force, or Bjerknes force, is a phenomenon whereby the momentum of an acoustic wave is imparted on a particle [60,61]. One application of this force is to concentrate microbubbles along a vessel wall by pushing them out of flow. This aggregation of microbubbles has been shown to improve imaging and drug delivery [62, 63, 64]. In another 
application termed acoustic radiation force impulse (ARFI) imaging, acoustic radiation force is used to displace tissue. This tissue displacement becomes a new source of image contrast and can be used to produce a functional map of tissue stiffness. A major limiting factor in displacement estimation performance during ARFI imaging is echo decorrelation. While signal processing methods can reduce decorrelation, it is also recognized that transducer design and imaging parameters play an important role in reducing the observed levels of echo decorrelation. As a result, there is a need for ultrasound transducers optimized for ARFI imaging. A transducer optimized for ARFI imaging may also enable improved microbubble translation and aggregation, thus improving imaging and drug delivery.

\subsection{Overview}

In Part 1 of this dissertation, I develop the concept of in situ microbubble production via microfluidic microbubble fabrication. By producing microbubbles directly within the vasculature, transiently-stable microbubbles can be utilized since issues regarding storage and systemic circulation are removed or reduced. With access to transient-stability, large diameter microbubbles can be designed in order to provide improved contrast enhancement and drug delivery as compared to small diameter microbubble formulations, without compromising safety.

In order to achieve in situ microbubble production, first a method to produce microbubbles that is capable of fitting within the vasculature must be developed. Microfluidics provides a framework to achieve this. Current designs, however, still require benchtop dimensions due to bulky interconnects between the tubing and the actual microfluidic inlets. To overcome this

problem, I present a new flooded method to minimize the interconnect footprint as described in Chapter 2.

Another advantage of microbubble production via microfluidic devices is the ability to adjust microbubble diameter and production rate in real-time. This could enable users 
to tune microbubbles in situ rather than trying to predict optimal microbubble size and concentration a priori. Furthermore, knowing the relationship between the gas and liquid input parameters and microbubble diameter could help guide microfluidic device design by providing bounds on the necessary gas pressure and liquid flow rates a device must withstand to achieve a certain microbubble diameter or production rate. In Chapter 3, a FFMD is extensively characterized in order to develop a model to predict microbubble production rate as a function of liquid and gas flow rate.

Biocompatibility is another important factor to consider when producing microbubbles directly in the vasculature since, in the simplest implementation, excess shell material will be released directly into the bloodstream. Albumin is the most abundant protein in plasma and has an established history as a microbubble stabilizer. In Chapter 4, albumin is investigated as a shell material for the microfluidic production of transiently-stable microbubbles that can be directly released into the bloodstream.

Next I explore the capabilities of in situ produced microbubbles for drug delivery enhancement. Utilizing a custom designed flow chamber, transiently-stable microbubbles produced in real-time from a microfluidic device are insonated in the presence of a model drug and cells as described in Chapter 5.

Finally, using the knowledge and experience gained from the previous chapters, the first application of microfluidic microbubbles directly administered to the mouse vasculature through a tail-vein catheter is reported. As described in Chapter 6, the output of a microfluidic device producing transiently-stable microbubbles in real-time is connected to the tail vein of a C57Bl/6 mouse. During microbubble administration, short-axis images of the right and left ventricle were obtained and image contrast within the ventricles was analyzed.

In Part 2, work related, but tangential, to the in situ microbubble production is described. First, work towards the development on an optimal transducer for acoustic radiation force impulse (ARFI) imaging is presented in Chapter 7. Although the focus is on ARFI imaging, the in silico designed transducer could also be utilized for microbubble applications requiring 
acoustic radiation force. Finally, in Chapter 8, work towards an improved ultrasound contrast agent that combines the longevity and payload of erythrocyte ghosts with the acoustic trigger of microbubbles is presented. 


\section{Part I}

\section{Microfluidics}




\section{Chapter 2}

\section{Liquid flooded flow-focusing}

\section{microfluidic device for in situ}

\section{generation of monodisperse}

\section{microbubbles ${ }^{1}$}

\subsection{Abstract}

Current microbubble-based ultrasound contrast agents are administered intravenously resulting in large losses of contrast agent, systemic distribution, and strict requirements for microbubble longevity and diameter size. In this chapter, I propose the concept of microbubble production directly within the vasculature, termed in situ microbubble production, as a means to avoid these limitations. Flow-focusing microfluidic devices (FFMDs) are a promising technology for enabling in situ production as they can produce microbubbles with precisely controlled diameters in real-time. While the microfluidic chips are small, the

${ }^{1}$ This chapter appears in the peer-reviewed publication:

A. H. Dhanaliwala, J. L. Chen, S. Wang, and J. A. Hossack, "Liquid flooded flow-focusing microfluidic device for in situ generation of monodisperse microbubbles," Microfluid Nanofluidics, vol. 14, no. 3-4, pp. 457-467, Mar. 2013. 
addition of inlets and interconnects to supply the gas and liquid phase greatly increases the footprint of these devices. Large footprints limit the potential to miniaturize FFMDs to sizes compatible with medium and small vessels. To overcome this challenge, a pressurized liquid-filled chamber is directly coupled to the liquid inlets of the FFMD, thus supplying the liquid phase without bulky interconnects. The microbubble diameter and production rate of FFMDs using this new method were measured optically over a range of gas pressures and liquid flow rates. The smallest FFMD manufactured measured $14.5 \times 2.8 \times 2.3 \mathrm{~mm}$. A minimum microbubble diameter of $8.1 \pm 0.3 \mu \mathrm{m}$ was achieved at a production rate of 450,000 microbubbles/s (MB/s). In addition, an intravascular ultrasound (IVUS) catheter was coupled to a flooded FFMD to produce an integrated IVUS-microbubble device. B-mode images of microbubbles produced in situ with a flooded FFMD in a gelatin phantom vessel were acquired to demonstrate the potential of real-time production and imaging. Microbubble production rates of $222,000 \mathrm{MB} / \mathrm{s}$ from a flooded FFMD within the vessel lumen provided a $23 \mathrm{~dB}$ increase in B-mode contrast.

\subsection{Background}

Microbubbles, comprising shell stabilized gas bubbles, are ideal ultrasound contrast agents as their high compressibility and low density make them highly echogenic. As a result, microbubbles have been developed to enable or to enhance a wide range of ultrasound applications including: detection of cardiac wall motion abnormalities [22, 65], molecularly targeted imaging [25, 26], targeted drug delivery [33, 31], blood perfusion measurements [24], and tumor coagulation therapy [66].

The most common method for microbubble production is agitation [67, 68, 17], in which a low solubility gas is dispersed within a liquid solution containing a stabilizing shell material. Agitation methods can produce large quantities of microbubbles $\left(\approx 10^{9}\right.$ microbubbles $/$ batch $)$; however, it is difficult to precisely control microbubble diameter and concentration with these 
methods.

As described in section 1.3 of the introduction, microfluidic devices direct gas and liquid phases through micrometer sized channels and apertures in order to use shear forces to produce microbubbles from a gas cone or jet. Several microfluidic device designs for microbubble production exist including: t-junction [53] and co-flow [47]. However, FFMDs [52, 56, 48, $49,51,50]$ have found preference as they can be produced at micrometer scales in a simple, low-cost, and time efficient fabrication process. FFMDs can be further categorized depending on the geometry of the outlet channel. In the expanding nozzle design, a velocity gradient develops as the gas and liquid phase pass through the narrow nozzle and into an expanding outlet [59]. As a result, the peak shear is confined to the narrow orifice thus reducing the flow rate at which microbubble pinch-off occurs. Since the volume of the gas bubbles from an FFMD, for a given gas and liquid flow rate, is consistent, microbubble diameter after each pinch-off event is consistent, resulting in a monodisperse population of microbubbles.

One factor that has limited adoption of microfluidic devices is low production rates [46] of vascular compatible microbubbles (i.e. $<10 \mu \mathrm{m}$ ). Current generation microbubbles such as DEFINITY ${ }^{\circledR}$ are dosed at approximately nine billion microbubbles for an average adult male with $98 \%$ of the microbubbles less than $10 \mu \mathrm{m}$ in diameter [78]. These quantities and diameter are required to compensate for losses that occur during administration and circulation $[20,79,80,81]$ and to reduce the risk of emboli [82]. Production rates of stable lipid shelled microbubbles from an FFMD are on the order of $10^{3}$ microbubbles per second $(\mathrm{MB} / \mathrm{s})[83,50]$, with production rates decreasing to as little as $10 \mathrm{MB} / \mathrm{s}$ as microbubble diameters approach sizes less than $10 \mu \mathrm{m}$ [70]. While the microbubble production rate of an FFMD can be increased by manipulating flow rates and fluid properties, this increase comes at the expense of reduced diameter control [51]. Production rates can also be increased by increasing the number of microbubble producing nozzles in a single FFMD [84, 85, 86, 87, 88], or by running several FFMDs simultaneously; however these parallelized devices can be difficult to operate. Equal pressures need to be supplied to each gas and liquid channel, 
which can require a complex 3D manifold [89] in order to maintain monodispersity, and it is difficult to evenly distribute the pressure and flow conditions necessary to produce uniform, small, microbubbles (i.e. $<10 \mu \mathrm{m})$ through all the channels. As a result, parallelized FFMD systems often produce microbubbles with large diameters (i.e. $>100 \mu \mathrm{m}$ ), large size variation between nozzles, or are limited to producing liquid in liquid microdroplets.

In order to overcome these limitations, I propose the concept of in situ microbubble production (i.e. microbubble production directly within the vasculature). By producing microbubbles within the vasculature, loss due to injection and circulation can be minimized or eliminated, thus significantly reducing the quantity of microbubbles necessary to produce contrast. Furthermore, as the microbubbles do not need to be stored, novel formulations can be investigated that include, for example, highly soluble but more biocompatible gases or lower stability shells. Most significantly, this relaxes the necessity for small (i.e. $<10 \mu \mathrm{m}$ ) microbubble diameters.

As described in appendix B, larger microbubbles are desirable since scattering cross section increases with diameter [90, 91, 92]. Furthermore, larger microbubbles have more surface area to carry a therapeutic agent or targeting ligand. Using unstable shells and biocompatible gases, in situ microbubble production could enable the production of large microbubbles (i.e. $>10 \mu \mathrm{m}$ ) for which dissolution can be relied upon to limit the emboli risk. Microfluidics also enables real-time adjustment of microbubble diameter and production rate to fit the clinical scenario. Unfortunately, in their current implementation FFMDs are difficult to miniaturize to the size necessary to fit within a vessel.

To enable further miniaturization, a new method for supplying the liquid phase to FFMDs, thus eliminating bulky interconnects, is described. Instead of supplying the liquid phase to all the inlets using a complex manifold, such as a 3D arrangement of discrete tubes (Fig. 2.1) or a maze of branching microfluidic channels [93], a pressurized liquid compartment is directly coupled to the liquid inlets of the FFMD (Fig. 2.2a, b). This design provides two main advances over current FFMD techniques. First, the number of tube-device interconnects are 


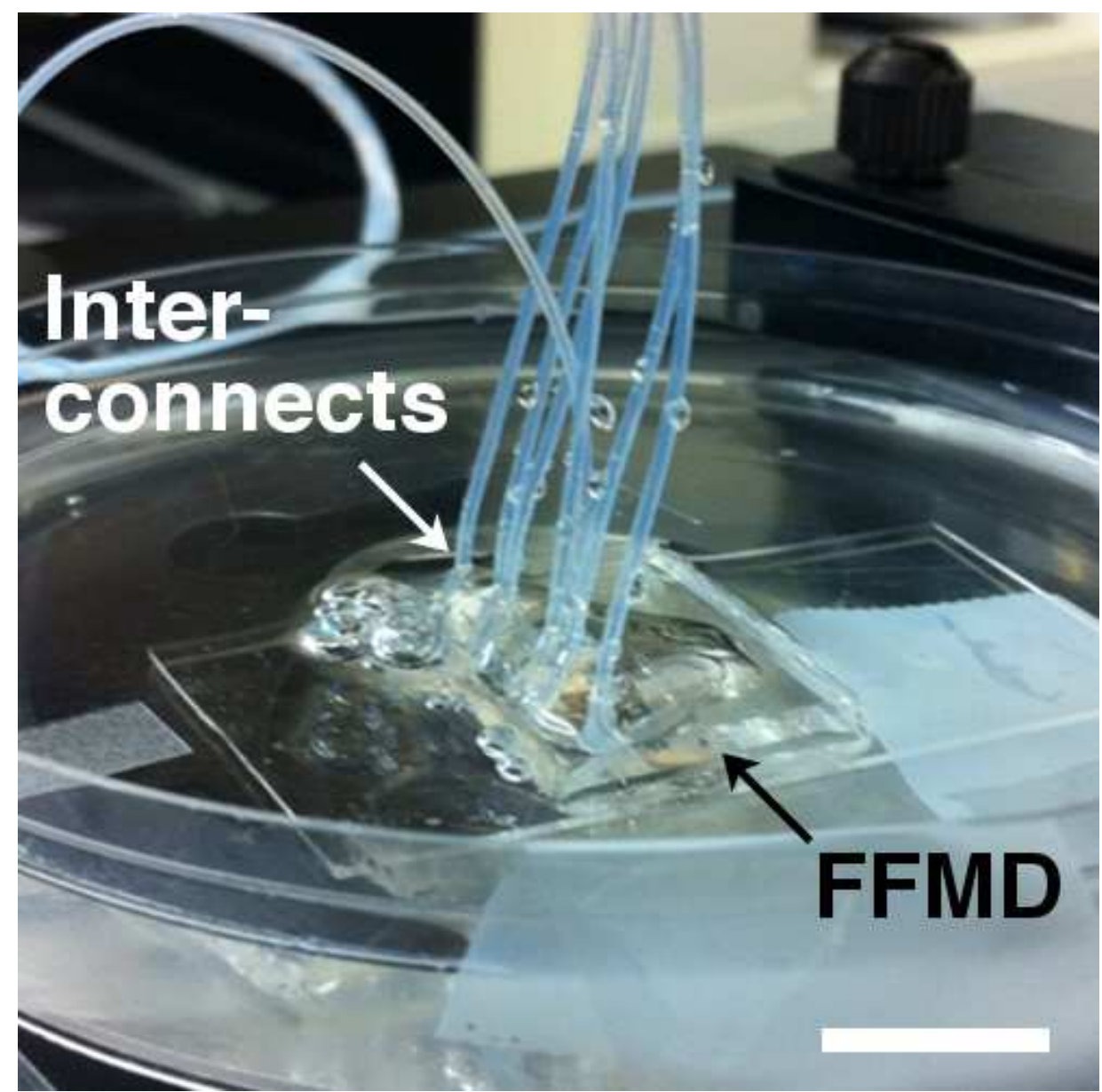

Figure 2.1: Bulky epoxy interconnects between tubing and the flow focusing microfluidic device (FFMD) inlets limits the ability to miniaturize microfluidic devices. Scale bar $=20 \mathrm{~mm}$.

reduced as the liquid inlets can be directly plasma bonded to the liquid chamber. This also allows the distance from the liquid inlets to the nozzle to be shortened as large inlets are not needed to accommodate the tubing interconnects. Furthermore, since a plasma bond is used, the connection is less likely to be clogged, as compared to epoxy bonds, or fail due to overpressure [94]. Second, and most important, by simplifying production and reducing the footprint of FFMDs, the flooded design facilitates miniaturization. By eliminating large bulky interconnects [95] and reducing the length of the microchannels, smaller FFMDs can be produced and, for example, be integrated into catheters intended for applications in the peripheral or coronary vasculature (i.e. $<5 \mathrm{~mm}$ in diameter).

In this chapter, I present a novel method for producing and operating FFMDs. Using 
an optimal concentration of a shell material dispersed in the liquid phase, the relationship between liquid flow rate, gas pressure, microbubble diameter, and microbubble production rate are characterized. Finally, I show proof-of-concept that in situ produced microbubbles can be imaged in real time using ultrasound.

\subsection{Materials and Methods}

\subsubsection{Microfluidic device fabrication}

Expanding nozzle flow-focusing microfluidic devices (FFMDs) were cast in poly-dimethylsiloxane (PDMS) (Sylgard 184, Dow Corning, Midland, MI) using a custom fabricated mold [57]. The custom molds were fabricated by spinning a negative photoresist (SU8-3025, Microchem, Newton, MA) onto a silicon wafer then exposing the wafer to UV through a custom designed, $0.1 \mu \mathrm{m}$ spot size quartz mask (Microtronics Inc, Newtown PA). Devices were designed to have the following features: $5 \mu \mathrm{m}$ wide nozzle, $5 \mu \mathrm{m}$ filters (Fig. 2.2 arrow) at all inlets to prevent clogging from PDMS debris [51], posts (Fig. 2.2 arrowhead) throughout the channels (36 and $160 \mu \mathrm{m}$ in diameter for the two-nozzle and single nozzle devices, respectively) to prevent channel collapse [96, 97], $35 \mu \mathrm{m}$ wide gas channel, $50 \mu \mathrm{m}$ wide liquid channels, and $20 \mu \mathrm{m}$ height. The gas phase entered the device via the gas inlet $(\mathrm{G})$. The liquid phase entered from the liquid inlets (L) and intersected with the gas at the expanding nozzle to produce microbubbles. PDMS in a 10:1 prepolymer to curing agent ratio was poured onto the mold, degassed, and then cured at $80^{\circ} \mathrm{C}$ for $30 \mathrm{~min}$.

Two types of flooded FFMDs were produced: externally mounted flooded FFMDs (eFFMDs) and internally mounted flooded FFMDs (iFFMDs) (Fig. 2.2c, d). Holes (0.65 mm) were drilled into a $10 \mathrm{~mm}$ square polystyrene cuvette (Fisher Scientific, Pittsburgh, PA) to create the liquid inlets ports for the eFFMDs or microbubble outlets ports for the iFFMDs. eFFMDs were then fabricated by plasma bonding (March II, Nordson March, Concord, CA) (60 W, 1 min) a PDMS device to the outside of a predrilled cuvette treated for 20 min with a 
1\% APTES solution (3-Aminopropyl)triethoxysilane, Sigma-Aldrich, St. Louis, MO) [98]. iFFMDs were fabricated by first plasma bonding a thin layer of PDMS (25 W $30 \mathrm{~s})$ to the microfluidic device before attaching the device to the interior of the cuvette with a two part epoxy (Hysol RE2039 and HD3561, Henkel Corp. Mooresville, NC). A 0.76 mm inner diameter PTFE tube (Polytetrafluoroethylene, Cole Parmer, Vernon Hills, IL) was then inserted into the gas inlet and sealed to the PDMS device with epoxy. Finally, a PTFE tube for supplying the liquid phase was placed inside the cuvette prior to sealing the chamber with epoxy and hot melt glue (McMaster-Carr Robbinsville, NJ). iFFMDs were also produced using a $3 \mathrm{~mm}$ square glass tube (S103, Vitrocom Mountain Lakes, NJ) and a $6 \mathrm{~mm}$ inner diameter glass pipette (Fisher Scientific, Pittsburgh, PA).

\subsubsection{Microbubble production}

The liquid phase consisted of $3 \mathrm{mg} / \mathrm{mL}$ polyethylene-glycol-40-stearate (PEG40st) (SigmaAldrich, St. Louis, MO), dispersed in a 10\% glycerol and 10\% propylene glycol solution (GPW). The solution was sonicated (XL2020, Misonix, Farmingdale, NY) with a half-inch probe (40\% power, $30 \mathrm{~min})$ to disperse micelles and large aggregates, and filtered through a $0.45 \mu \mathrm{m}$ syringe filter (Fisher Scientific, Pittsburgh, PA) prior to use to remove any additional aggregates. The gas phase consisted of $99.998 \%$ pure nitrogen gas (GTS Welco, Richmond, VA). The liquid phase was supplied to the device using a syringe pump (PHD2000, Harvard Apparatus) at flow rates between 10 and $180 \mu \mathrm{L} / \mathrm{min}$ while the gas phase was applied at pressures between 34.5 and $103 \mathrm{kPa}$. Pressure was set and maintained using a two-stage regulator (VTS 450D, Victor Technologies International, Inc., St. Louis, MO) and the pressure at the gas inlet was verified using a digital manometer.

Microbubbles were produced by first applying the liquid phase at the flow rate under investigation until the liquid pressurized chamber and all FFMD channels were completely flooded. The gas phase was then applied and maintained at the pressure under investigation. Once stable microbubble production was achieved, images of the microbubbles were captured 


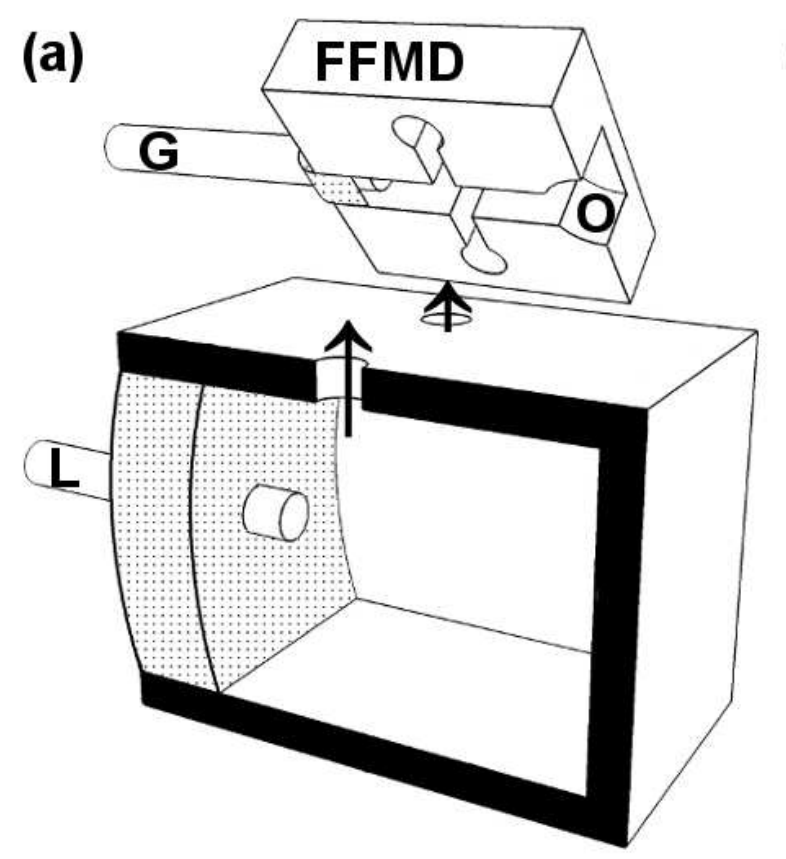

\section{(b)}
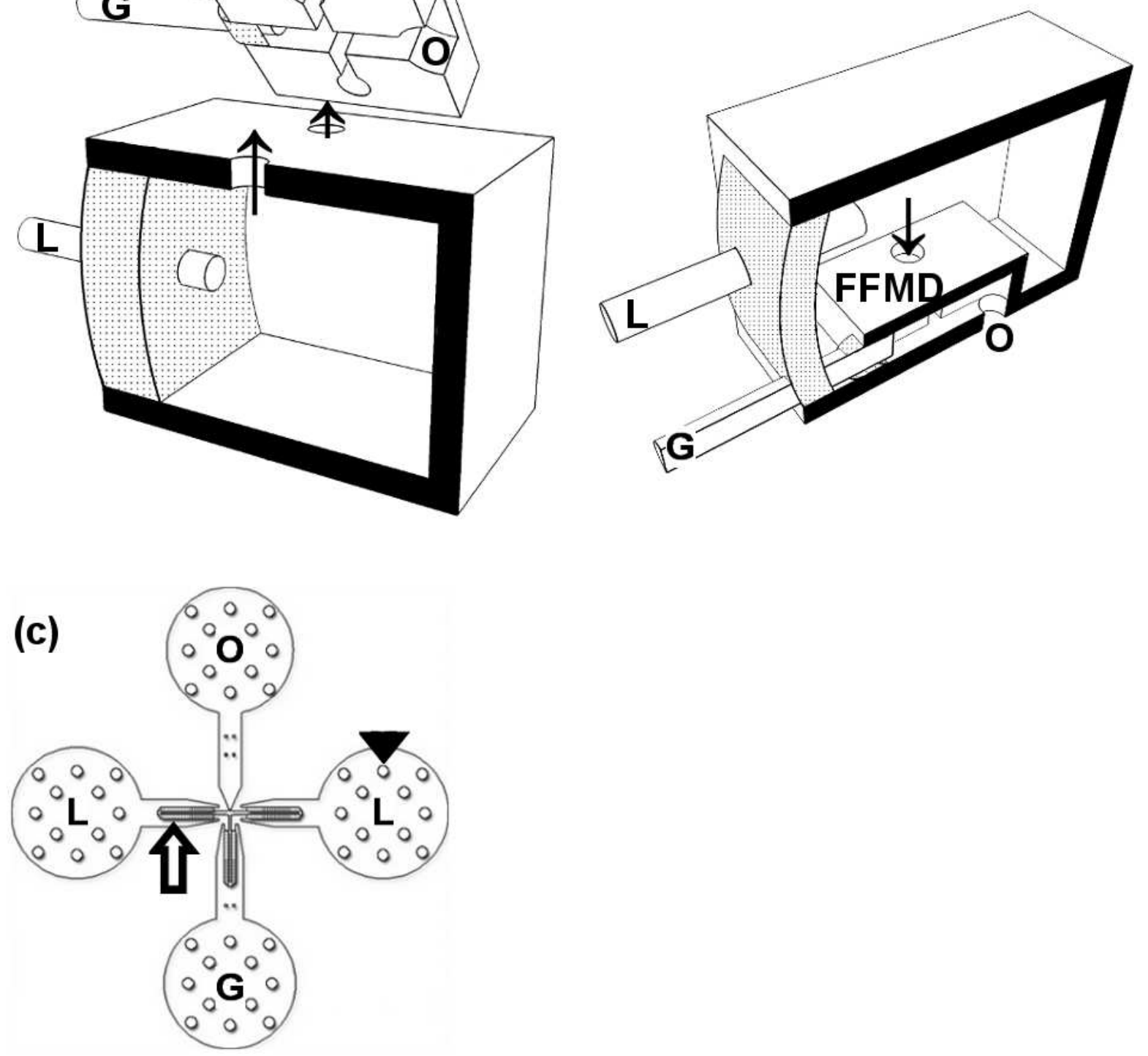

Figure 2.2: Schematics for (a) externally mounted (eFFMD) and (b) internally mounted (iFFMD) flooded flow-focusing microfluidic devices. (G: gas inlet; L: liquid inlet; O: microbubble outlet; FFMD: flow-focusing microfluidic device. Black indicates cut plane, dotted indicates epoxy. Not to scale). For both designs, gas is provided directly to the device via tubing while the liquid phase is pumped into a separate chamber, pressurizing the liquid, and ultimately forcing it through the ports connecting the chamber and the FFMD (arrows). (c) Mask design for the flow-focusing microfluidic designs. Features include posts within the channels to prevent channel collapse (arrow head), and filters at the gas and liquid inlets to prevent debris from entering the flow-focusing nozzle (open arrow). 
using an inverted microscope connected to a high speed framing camera (SIMD24, Specialised Imaging, Simi Valley, CA) operating at up to 20 million frames per second. Microbubble diameter and production rate were measured from the acquired images using ImageJ (v 1.46d NIH Bethesda, MD). The relationship between gas pressure, liquid flow rate, microbubble diameter, and production rate was investigated by incrementing the liquid flow rate in $2 \mu \mathrm{L} / \mathrm{min}$ step sizes while maintaining a constant gas pressure. Microbubble diameter and production rate were then measured after each increment in flow rate. Measurements were repeated until the FFMD no longer stably produced monodisperse microbubbles. The gas pressure was then incremented, liquid flow rate reset, and the process repeated. Data was only collected for gas and liquid combinations that resulted in microbubbles less than $20 \mu \mathrm{m}$ in diameter (i.e. less than the height of the FFMD channels) to limit the effect of wall interaction on microbubble production dynamics.

\subsubsection{Acoustic imaging}

A gelatin phantom ${ }^{2}$ with a $1.79 \mathrm{~cm}$ diameter lumen was constructed to simulate a vessel. Microbubble production rate and diameter size from the FFMD was verified optically prior to insertion into the phantom lumen. An oscillatory flow of deionized water was established through the lumen using a peristaltic pump (Unispense 340, Wheaton Industries, Millville, NJ). The manufactured microbubbles were imaged inside the vessel using a linear array connected to a clinical ultrasound scanner (Siemens Sequoia 512 Scanner and 15L8 transducer, Siemens Medical Solutions, Malvern, PA). The microbubbles were imaged at a mechanical index (MI) of 0.2 and a center frequency of 14 or $7 \mathrm{MHz}$ when using either fundamental B-Mode or a contrast agent specific nonlinear imaging mode (Contrast Pulse Sequences [CPS] [99]), respectively. Flow was stopped and the MI was set to 1.9 on the linear array while in CPS mode in order to investigate microbubble destruction. In situ manufactured microbubbles were also imaged with a coupled IVUS-FFMD using a $45 \mathrm{MHz}$ intravascular

260 g gelatin (Type B, Fisher Scientific) and $10 \mathrm{~g}$ agar (Fisher Scientific) per liter water. Boil water prior to adding gelatin and agar. Additional amounts of graphite can be added to increase speckle. 
ultrasound (IVUS) catheter connected to a clinical IVUS imaging system (InVision Gold Scanner and Revolution catheter, Volcano Corp. San Diego, CA) (Fig. 2.7a).

\subsection{Results and discussion}

\subsubsection{FFMD fabrication and shell material optimization}

Both externally mounted flooded FFMDs (eFFMDs) and internally mounted flooded FFMDs (iFFMDs) were successfully fabricated (Fig. 2.3). The final nozzle and filter widths were $8 \mu \mathrm{m}$. All other dimensions were produced as designed. The simplification of construction allowed for a significant reduction in FFMD size. The smallest iFFMD manufactured to date measures $14.5 \times 2.8 \times 2.3 \mathrm{~mm}$. While eFFMDs and iFFMDs share the same fundamental design - a common liquid pressurized chamber in order to apply a constant liquid pressure across all liquid inlets - each device has advantages and disadvantages. The iFFMD requires fewer drilled holes in the wall of the pressurized chamber. Alignment is easier as only the microbubble outlet port needs access to the outside. eFFMD, however, requires access to each liquid inlet so multiple holes need to be aligned to the ports. The most important difference is the pressure applied to the microfluidic device. In eFFMDs, the channels in the microfluidic device are not subject to the pressures inside the liquid chamber while in the iFFMD, the microfluidic device needs to withstand the pressure necessary to drive liquid through the inlets.

Initial testing of the FFMDs used the surfactant Tween 20 (Sigma-Aldrich, St. Louis, MO) in the liquid phase to stabilize the microbubble shell [52, 48, 49]. Concentrations of Tween above $2 \mathrm{mg} / \mathrm{mL}$ were required to produce stable microbubbles at a high production rate; however, concentrations of Tween 20 as low as $0.5 \mathrm{mg} / \mathrm{mL}$ are known to lyse cells (SigmaAldrich 2012). Phospholipids such as 1,2-distearoyl-sn-glycero-3-phosphocholine (DSPC) (Avanti Polar Lipids, Alabaster, AL) are also commonly used as a shell material to stabilize the microbubble $[51,70,50]$ and were investigated. Solutions containing $2 \mathrm{mg} / \mathrm{mL}$ DSPC and 


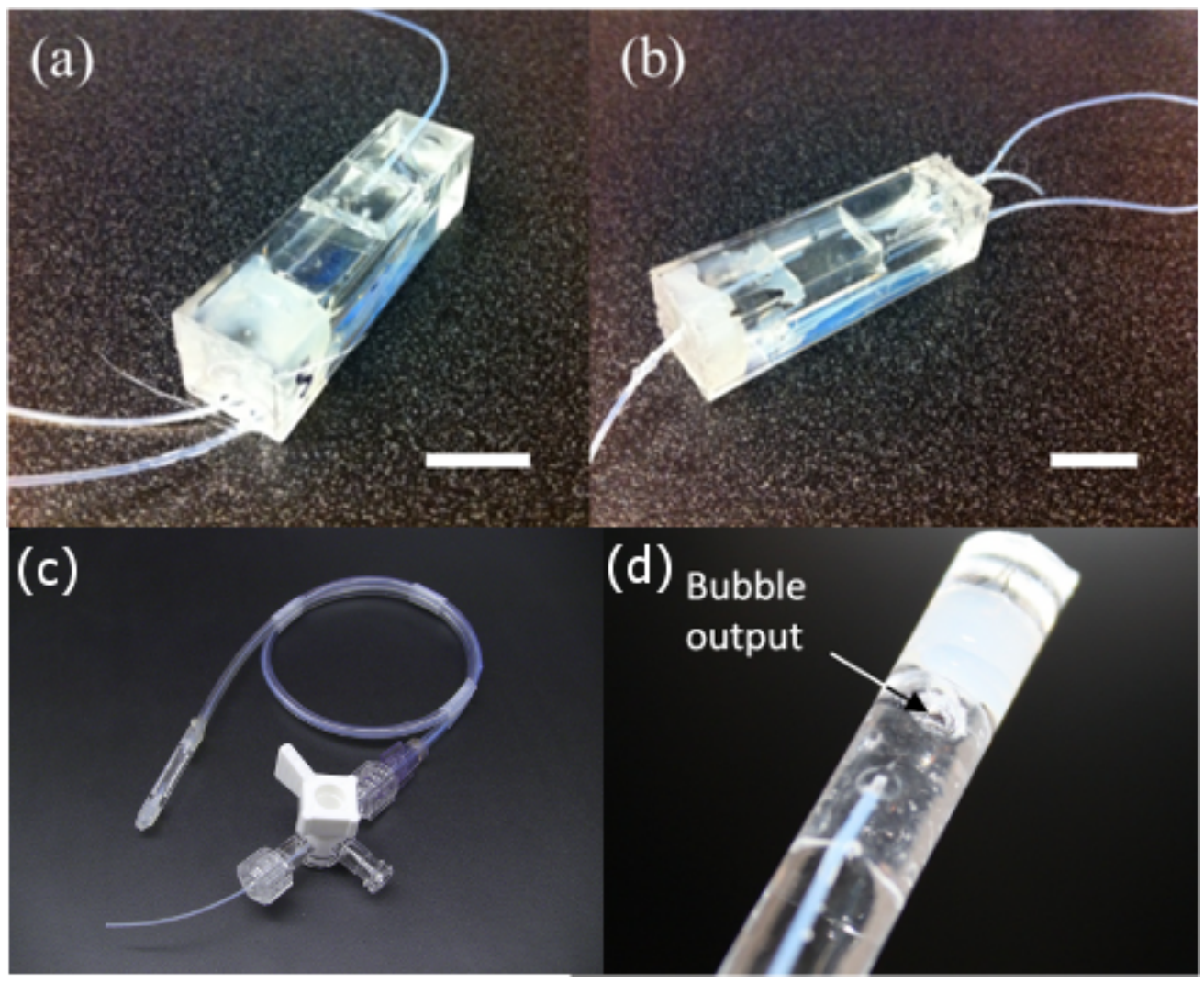

Figure 2.3: (a) eFFMD and (b) iFFMD. Scale bar $=1 \mathrm{~cm}$. (c) Model "catheter" for in situ microbubble production. (d) Zoom of iFFMD located within the "catheter" tip.

$2 \mathrm{mg} / \mathrm{mL}$ PEG40st resulted in microbubble coalescence when production rates were increased above 90,000 microbubbles/s (MB/s) or when producing microbubbles smaller than $20 \mu \mathrm{m}$ in diameter. Increasing the concentration of DSPC to $20 \mathrm{mg} / \mathrm{mL}$ eliminated coalescence and allowed microbubbles as small as $12 \mu \mathrm{m}$ to be produced. However, at this concentration, production rates peaked at 3,900 MB/s - likely a consequence of the increased liquid phase viscosity. Consequently, in order to obtain the high production rates of Tween 20 and the biocompatibility of lipid, PEG40st in a glycerol propylene glycol solution [51, 50] was selected as the shell material.

The optimal PEG40st concentration was determined to be $3 \mathrm{mg} / \mathrm{mL}$. Microbubbles produced with PEG40st solutions at concentrations below $2.5 \mathrm{mg} / \mathrm{mL}$ coalesced immediately upon formation. Cells incubated with concentrations of PEG40st below $2 \mathrm{mg} / \mathrm{mL}$ exhibited no membrane permeabilization, while cells incubated with concentrations of PEG40st above 
$3 \mathrm{mg} / \mathrm{mL}$ exhibited complete membrane permeabilization. Although PEG40st at concentrations of $3 \mathrm{mg} / \mathrm{mL}$ caused cell permeabilization, only small volumes $(<200 \mu \mathrm{L} / \mathrm{min})$ of the liquid phase would be exiting the microfluidic device during production. As a result, the PEG40st should quickly be diluted to concentrations that do not cause permeabilization. PEG40st stabilized microfluidic microbubbles were not as stable as microfluidic microbubbles stabilized with lipid [51, 50], and exhibited a loss of polydispersity within $60 \mathrm{~s}$ after production.

\subsubsection{Microbubble production and characterization}

Both iFFMD and eFFMD were capable of producing monodisperse microbubbles. Microbubble production could be divided into five distinct regimes [100, 101] (Fig. 2.4), with the boundaries between the regimes determined by the transition of production from one type of microbubble to another. The flow rate limits that defined each of these regimes depended on gas pressure. Overall, increases in the gas pressure increased the minimum flow rate at which a regime began as well as broadened the range of flow rates over which a regime occurred. Microbubble production was most stable when the produced microbubbles had diameters less than the height of the channel but greater than the nozzle width - termed the stable regime. In addition, maximum production rate always occurred within the stable regime. As a result, flooded FFMDs were only operated and characterized in this regime. While it was possible to produce microbubbles with diameters that were smaller than the width of the nozzle, microbubble production would become unstable, production rate dropped precipitously, and microbubble multiplets (i.e. one or more microbubbles of different diameters produced in quick succession followed by a delay in microbubble production) began to form. Microbubbles with diameters larger than the height of the channel were not characterized in order to limit the effect of the wall on microbubble production dynamics.

While eFFMDs were capable of stably producing microbubbles at high production rates, iFFMDs were only able to produce microbubbles for short periods of time $(\approx 10 \mathrm{~min})$ during 

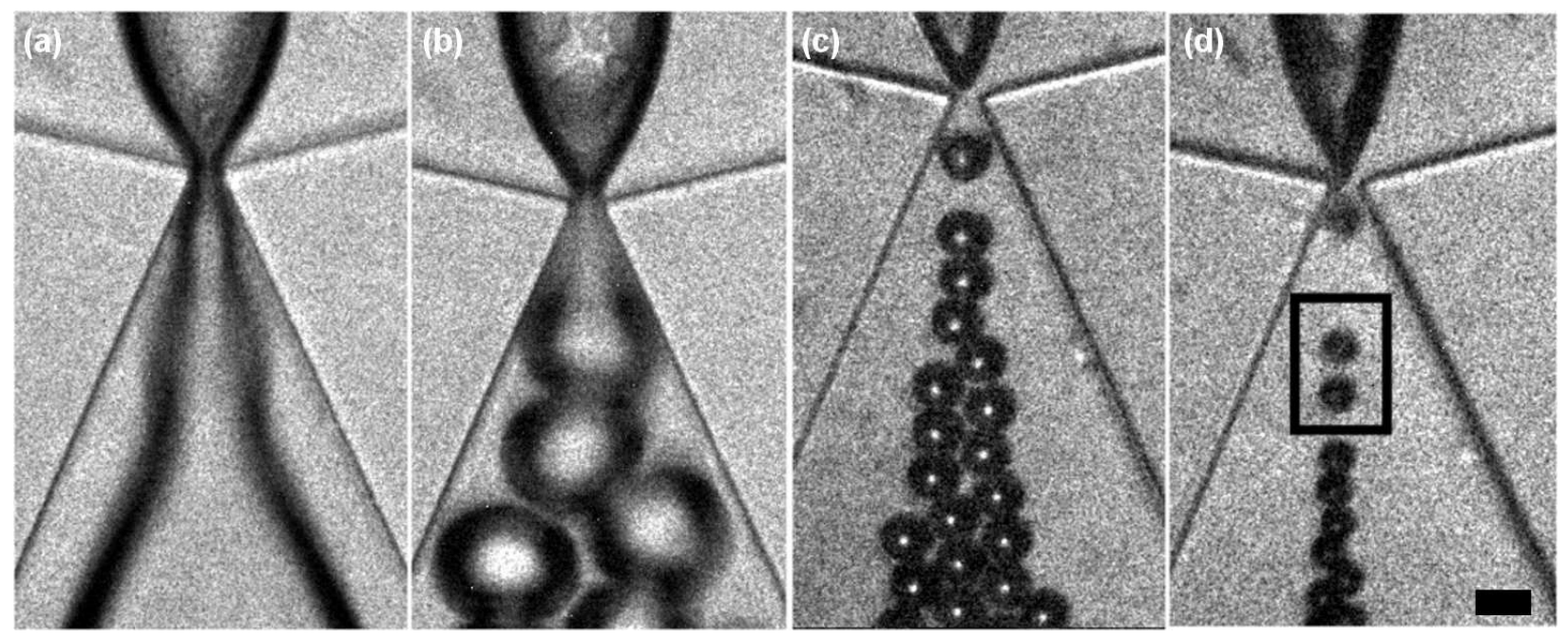

Figure 2.4: Microbubble production regimes of flooded FFMDs. Scale bar $10 \mu \mathrm{m}$ for all figures. (a) I: Overpressure $(<34 \mu \mathrm{L} / \mathrm{min})$, the gas pressure overwhelms the liquid flow rate and no microbubbles are produced. (b) II: Cylindrical $(34-60 \mu \mathrm{L} / \mathrm{min})$, microbubbles are larger than the height of the microfluidic channel resulting in the production of non-spherical microbubbles. (c) III: Stable (60-80 $\mu \mathrm{L} / \mathrm{min})$, microbubbles with diameters less than the height of the channel but greater than the nozzle width are stably produced. (d) IV: Unstable (80-92 $\mu \mathrm{L} / \mathrm{min})$, microbubble production becomes unstable and can result in doublet formation (i.e. two microbubbles in quick succession followed by a delay in microbubble production, black box) and microbubbles with diameters less than the nozzle width. V: Underpressure $(>92 \mu \mathrm{L} / \mathrm{min})$, liquid flow overwhelms the gas pressure and no microbubbles are produced (not shown). Flow rates are representative for a gas pressure of $55.2 \mathrm{kPa}$. The flow rate range for each regime, however, changes with gas pressure, with regime III broadening with increasing gas pressure.

which microbubble diameter would decrease until production ceased - despite liquid flow rate and gas pressure remaining constant. However, if the liquid flow rate remained constant, progressively increasing gas pressure over time rescued microbubble production. One possible cause for the observed behavior was that the increasing pressure inside the liquid chamber compressed the compliant PDMS gas channel. This would cause gas flow rate to decrease, resulting in smaller microbubbles, until flow ceases altogether, and is consistent with our observations. The eFFMD design mitigates the problem by placing the FFMD outside the pressurized liquid chamber and thus preventing the collapse of the gas channel.

eFFMDs were characterized at gas pressures between 48 and $75 \mathrm{kPa}$. The minimum flow rate and the range of flow rates in which the eFFMD stably produced microbubbles smaller than the channel height increased as gas pressure increased. The minimum flow rate increased 
Table 2.1: Microbubble production capabilities for an iFFMD and eFFMD

\begin{tabular}{ccc}
\hline & iFFMD & eFFMD \\
\hline \hline Smallest diameter $(\mu \mathrm{m})$ & $9.8 \pm 0.5$ & $8.1 \pm 0.3$ \\
Production rate @ smallest diameter $\left(10^{3} \mathrm{MB} / \mathrm{s}\right)$ & 3.6 & 450 \\
Flow rate $(\mu \mathrm{L} / \mathrm{min})$ & 10 & 108 \\
Gas pressure $(\mathrm{kPa})$ & 34.5 & 68.9 \\
Max production rate $\left(10^{3} \mathrm{MB} / \mathrm{s}\right)$ & 4.6 & 660 \\
Diameter @ max production rate $(4 \mu \mathrm{m})$ & $16.5 \pm 0.8$ & $9.5 \pm 0.6$ \\
Flow rate $(\mu \mathrm{L} / \mathrm{min})$ & 10 & 116 \\
Gas pressure $(\mathrm{kPa})$ & 41.4 & 75.8 \\
\hline
\end{tabular}

by $21 \mu \mathrm{L} / \mathrm{min}$ and the range over which the eFFMD operated increased by $27 \mu \mathrm{L} / \mathrm{min}$ between the lowest and highest gas pressures. For a given gas pressure, microbubble diameter decreased with increasing liquid flow rate (Fig. 2.5), with the smallest microbubble produced just prior to transition out of the stable regime. The smallest microbubble capable of being produced at a given pressure decreased for increasing pressures, with a plateau as the microbubble diameter approached the nozzle width. Production rate at a given gas pressure increased with increasing liquid flow rate (Fig. 2.5), peaking just prior to the transition out of the stable regime. The maximum production rate for a given pressure also increased with increasing pressure. These trends suggest that operating eFFMDs at higher gas pressures is optimal as it results in smaller microbubbles, higher production rates, and a wider range of liquid flow rates at which the device can operate stably. The last property provides improved control over microbubble diameter by increasing the separation between the liquid flow rates necessary to produce a microbubble of a specific size. Overall, the diameter of the smallest microbubble produced was $8.1 \mu \mathrm{m}$ and the maximum production rate was 660,000 microbubbles/second $(\mathrm{MB} / \mathrm{s})$ (Table 2.1).

\subsubsection{Imaging In situ produced microbubbles}

Microbubbles produced in situ by an eFFMD were successfully imaged in real time using a 128 element linear ultrasonic imaging array (Siemens 15L8) at $14 \mathrm{MHz}$ in B-mode and 


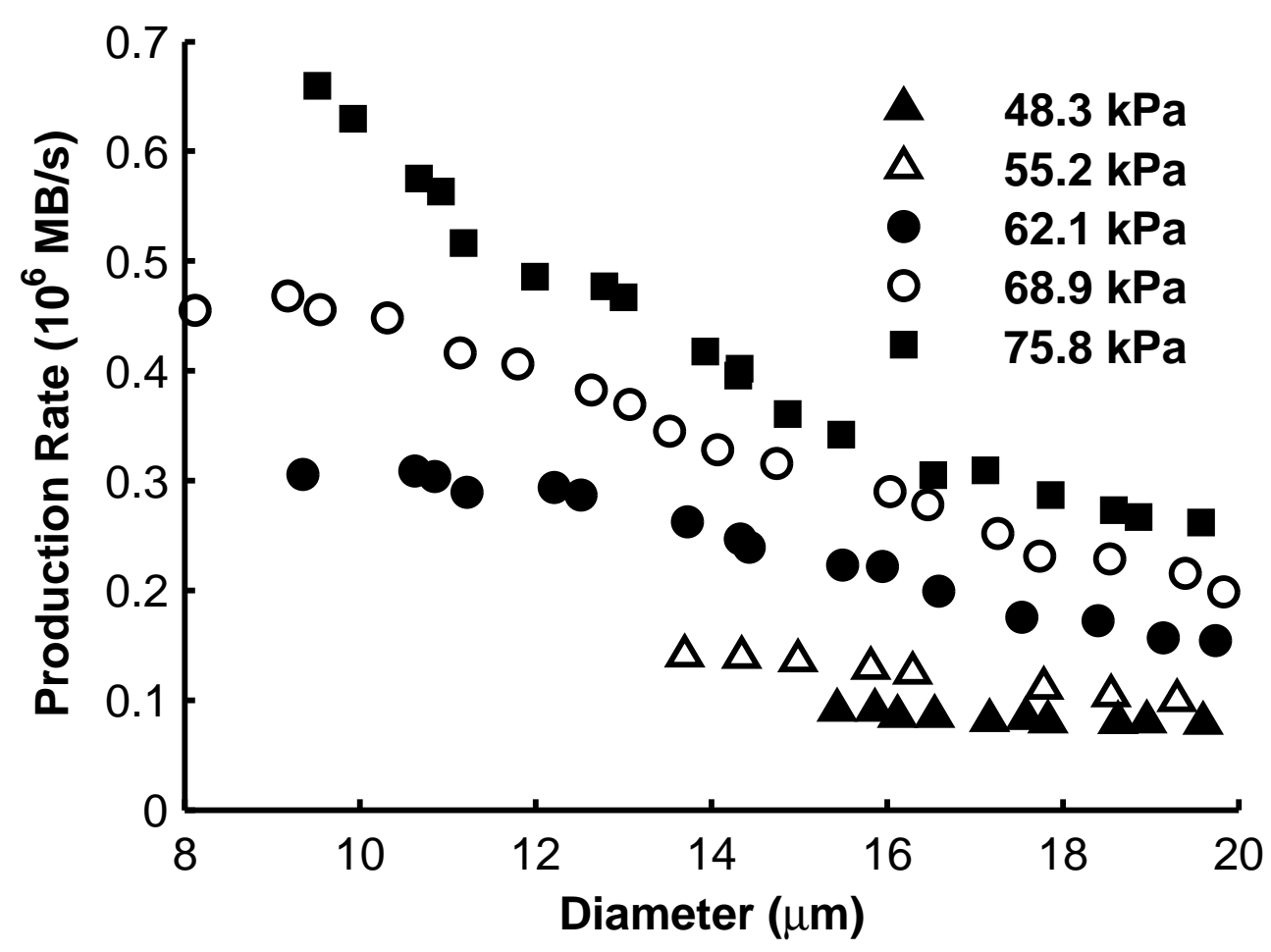

Figure 2.5: Diameter and production rate relationship of eFFMD produced microbubbles as a function of liquid flow rate and gas pressure. For a given gas pressure, liquid flow rate decreases from left to right. Diameters were measured optically and are an average of independent runs $(\mathrm{n}=3)$. Increasing gas pressure allowed progressively smaller microbubbles, approaching the nozzle width $(8 \mu \mathrm{m})$, at higher production rates, to be produced.

$7 \mathrm{MHz}$ in CPS mode (Fig. 2.6). The microbubbles were produced at a rate of 222,000 MB/s, with a diameter of $14 \mu \mathrm{m}$, and were acoustically active along the entire $13 \mathrm{~cm}$ length of the vessel. Improvements in image intensity were measured directly from 8-bit B-mode images that were acquired by digitizing the S-VHS output from the ultrasound scanner. A region of interest (ROI) placed within the lumen had a value of $5.3 \pm 3.6$ when the lumen was water filled and an intensity of $95.1 \pm 8.8$ during microbubble production. Microbubbles, therefore, provided an approximately 90-fold increase in the image intensity of the lumen ${ }^{3}$ (Fig. 2.6a, b). The eFFMD produced microbubbles also exhibited a strong acoustic response when imaged in CPS mode ( $\approx 157$-fold increase) and all microbubbles within the transducer field of view were destroyed $30 \mathrm{~s}$ after increasing the MI to 1.9 (Fig. 2.6c, d). When imaging at $45 \mathrm{MHz}$

\footnotetext{
${ }^{3}$ Since the images are log compressed, the difference between mean intensities is a more appropriate metric
} and is equivalent to the log of the ratio of the uncompressed image intensities. 
using an IVUS catheter and imaging system, 8-bit images were extracted for analysis from the acquired DICOM files. The microbubble-filled lumen displayed a $\approx 113$-fold increase over the water-filled lumen even though the imaging frequency was approximately two orders of magnitude larger than the expected resonant frequency of the microbubbles (Fig. 2.7b).

To date, microfluidic devices for microbubble production have been designed to produce stable microbubbles that can be stored and utilized in the future, similar to the way approved formulations of microbubbles are used [78]. Taking a different approach, in this chapter, I describe an attempt to utilize microfluidic devices for the production of microbubbles intended for immediate use. The flooded design enhances this capability by providing a means to reduce the dimensions of the microfluidic device to fit within the lumen of human vessels. Previous methods for producing microbubbles from microfluidic devices required viscous solutions of lipids and slow production rates to produce monodisperse microbubbles that could survive days to months [50]. By producing microbubbles in situ and imaging them immediately, shell formulation that are less stable but allow for much higher microbubble production rates can be used. While these rates may not be as high as those achieved by agitation methods, rates of 200,000 MB/s were more than adequate to provide significant and sufficient vessel contrast, as observed in the ultrasound images.

Catheter-based FFMDs are one application that could benefit from the advantages of the flooded FFMD design. Catheter-based FFMDs hold promise for in situ production of microbubbles within the vessel of interest, allowing for the production, imaging, and destruction of microbubbles within the immediate vicinity of a therapeutic site [102]. In situ production also significantly reduces the need for requiring microbubble stability/longevity. Microbubbles produced directly within vessels would only need to survive a few seconds, and over a few millimeters of transport to provide useful contrast or therapeutic effect (e.g. drug delivery). Consequently, unconventional microbubble designs, such as high solubility gas cores or unstable shell compositions, become feasible and possibly advantageous. For example, fluorocarbon gas may be replaced with oxygen, and gas dissolution [103, 21] away 


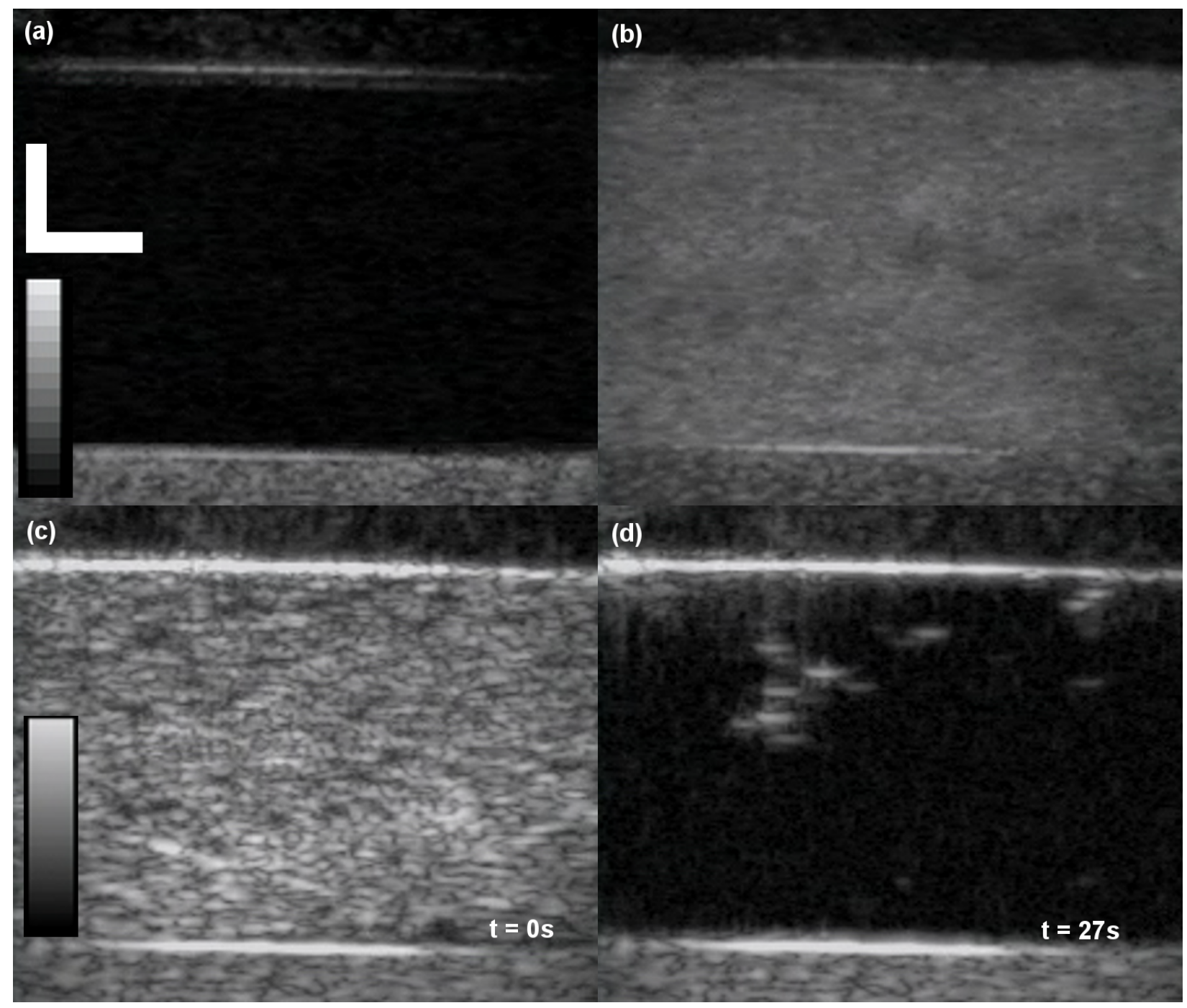

Figure 2.6: Ultrasound images of PEG40St microbubbles produced in situ via an eFFMD and imaged in real time with a 128 element linear array. (a) B-mode image $\left(\mathrm{f}_{c}=14 \mathrm{MHz}, \mathrm{MI}=0.2\right)$ of the $1.79 \mathrm{~cm}$ inner diameter lumen within the gelatin phantom before microbubble production. Top row dynamic range scale $=100 \mathrm{~dB}$ and gain $=0 \mathrm{~dB}$. (b) B-mode image of $14 \mu \mathrm{m}$ diameter microbubbles produced at a rate of $222,000 \mathrm{MB} / \mathrm{s}$. Microbubbles were under an $8 \mathrm{~cm} / \mathrm{s}$ peristaltic flow. (c) Contrast pulse sequence (CPS) mode $\left(\mathrm{f}_{c}=7 \mathrm{MHz}\right)$ image of the microbubbles. Bottom row dynamic range scale $=100 \mathrm{~dB}$ and gain $=20 \mathrm{~dB}$. Flow was temporarily stopped and the MI was increased to 1.9 in order investigate microbubble destruction. Complete destruction of all the microbubbles within the transducer field of view was observed after $30 \mathrm{~s}$ of insonation (d). Scale bar $5 \mathrm{~mm}$ in both directions and applies to all images.

from the target can be relied upon to prevent the formation of a distant gas emboli. As a result, the production of larger $(>10 \mu \mathrm{m})$ microbubbles, with increased scattering cross section and larger drug payload, may be envisaged without compromising safety. The flooded FFMD design would also help eliminate bulky interconnects allowing the catheter to be 

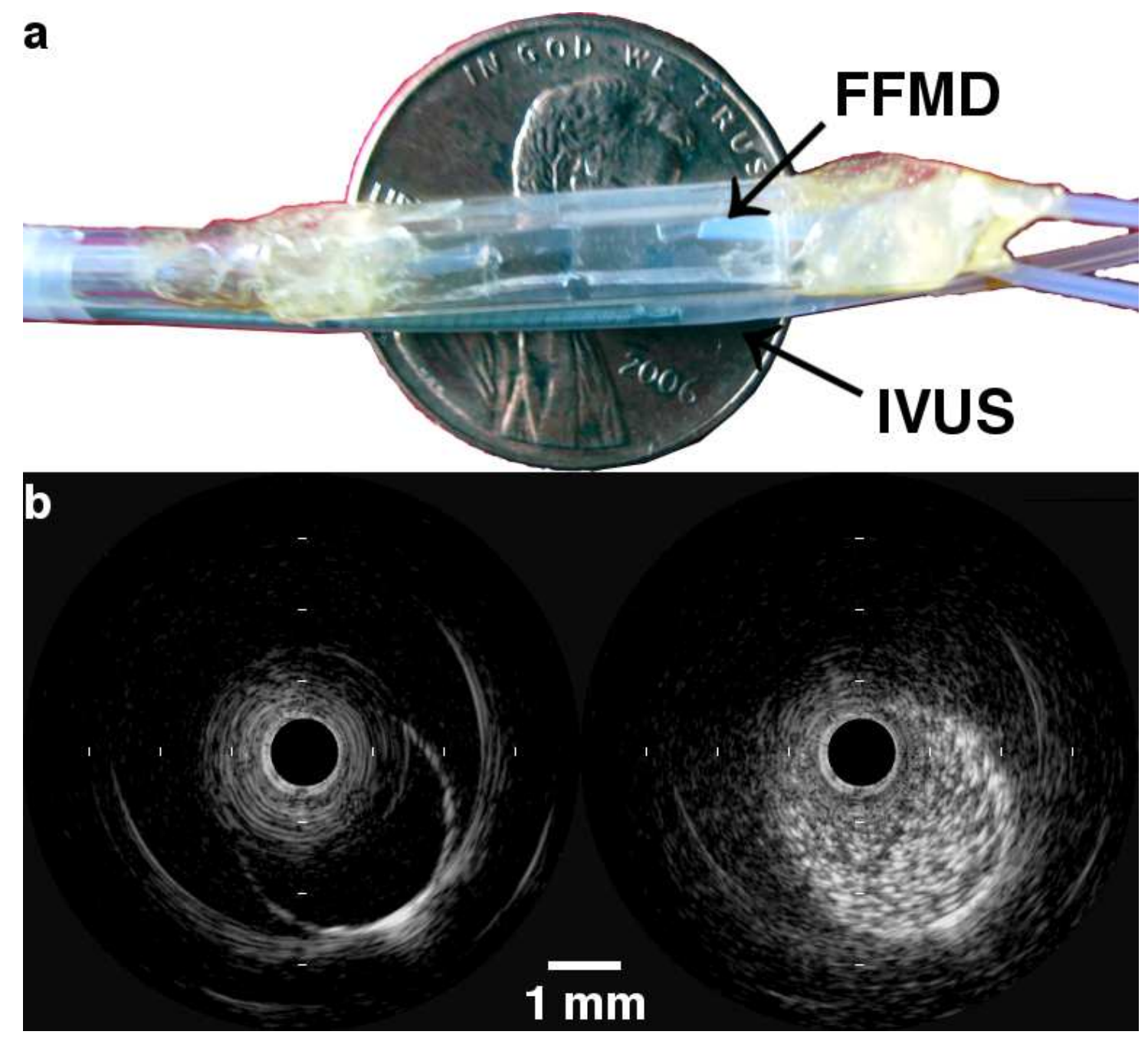

Figure 2.7: (a) Flooded FFMD coupled to a $45 \mathrm{MHz}$ IVUS catheter. (b) Phantom lumen imaged with a $45 \mathrm{MHz}$ IVUS catheter pre (left) and post (right) in situ microbubble production. A 113-fold increase in signal intensity was measured in the lumen post-contrast.

smaller, which is necessary for a successful catheter-based microbubble delivery system.

\subsection{Conclusion}

In this chapter, I described the fabrication and operation of a new method for supplying the liquid phase to a flow-focusing microfluidic device (FFMD). By coupling the device to a chamber containing a pressurized liquid phase, complex manifolds and unnecessary tubing interconnects were eliminated. Flooded FFMDs were shown to produce microbubbles as small 
as $8 \mu \mathrm{m}$ and at production rates as high as 660,000 microbubbles/second. These sizes and production rates were shown to produce excellent ultrasound contrast when produced in situ and immediately imaged. Ultimately, this design may simplify FFMD miniaturization and contribute towards the long-term goal of developing a therapeutic intravascular ultrasound catheter capable of in situ microbubble production, imaging, and drug delivery. 


\title{
Chapter 3
}

\section{Characterization of an}

\section{expanding-nozzle flow-focusing}

\author{
microfluidic devices for the
}

\section{development of a model to predict}

\section{production rate as a function of liquid}

\section{flow rate and gas pressure ${ }^{1}$}

\subsection{Abstract}

A unique aspect of flow-focusing microfluidic devices (FFMDs) is the ability to adjust microbubble diameter and production in real-time via changes to the gas and liquid input parameters. In this chapter, the microbubble diameter and production rate of an expanding-

${ }^{1}$ This chapter appears in the peer-reviewed publication:

S. Wang, A. H. Dhanaliwala, J. L. Chen, and J. A. Hossack, "Production rate and diameter analysis of spherical monodisperse microbubbles from two-dimensional, expanding-nozzle flow-focusing microfluidic devices," Biomicrofluidics, vol. 7, no. 1, pp. 014103-014103-12, Jan. 2013. 
nozzle FFMD is characterized and analyzed in order to develop a relationship between FFMD input parameters and microbubble production. Microbubble generation was observed to transition through five regimes as liquid flow rate increased at a constant gas pressure. The behavior of the gas cone tip was further analyzed and separated into two characteristic phases: bubbling and waiting. The duration of the bubbling phase was linearly related to the liquid flow rate, while the duration of the waiting phase was related to both liquid flow rate and gas pressure. Overall, the microbubble production rate was found to be inversely proportional to the sum of the bubbling and waiting times.

\subsection{Background}

As described in sections 1.2 and 1.3 of the introduction, microbubbles are the most common ultrasound contrast agent, and microfluidics is one alternative method to agitation for the production of microbubbles. With microfluidics, microbubble production rates and diameters can be measured directly using high speed video microscopy [51, 52] or via a Coulter counter. Neither of these methods are currently compatible with real-time in situ microbubble production since both require instruments that are difficult or impossible to scale to vascular compatible dimensions. Knowledge of microbubble concentration is important for imaging applications to help avoid imaging artifacts, and for drug delivery applications, to help calculate therapeutic drug dose. Furthermore, knowledge of required gas pressure and liquid flow rate to achieve a specific microbubble diameter or production rate can help guide microfluidic device design by setting stress boundaries that the device must withstand. Consequently, reliable models that can predict microbubble production rate in response to changes in geometry, gas pressure, and liquid flow rates are important for facilitating widespread adoption of microfluidic devices as the models can guide microfluidic device development and operation. Given the benefits of flow-focusing microfluidic devices (FFMDs), as described in section 2.2, the focus of this chapter is on developing a relationship between the 
input parameters and the production rate of microbubbles produced via an expanding-nozzle FFMD design.

\subsubsection{Theory}

Previous studies $[108,47,109,106,110]$ have shown that the breakup frequency of a gas jet into microbubbles in a capillary flow-focusing device with a circular cross-section nozzle and a constant gas flow rate can be described by:

$$
\omega \propto \frac{Q_{l}}{d_{j} D^{2}},
$$

For FFMDs with a short-channel nozzle and a rectangular cross-section generating Tween stabilized microbubbles, Garstecki et al. [48, 111] showed that the time that the gas thread stayed open at the nozzle (i.e. $\tau$ ) was inversely proportional to liquid flow rate:

$$
\tau \propto \frac{1}{Q_{l}}
$$

While both these relationships hint at the microbubble production rate from a microfluidic device, no model has been specifically developed to describe microbubble production rate for 2-D expanding-nozzle FFMDs.

In this chapter, a 2-D expanding-nozzle FFMD was fabricated and microbubble diameter and production rates were measured over a range of gas pressures and liquid flow rates. In order to facilitate comparison with previous work, Tween - a surfactant and widely used shell material $[48,49,112,52]$ — was used to stabilize the microbubbles. An empirical relationship between microbubble production rate as a function of input parameters was then developed. 


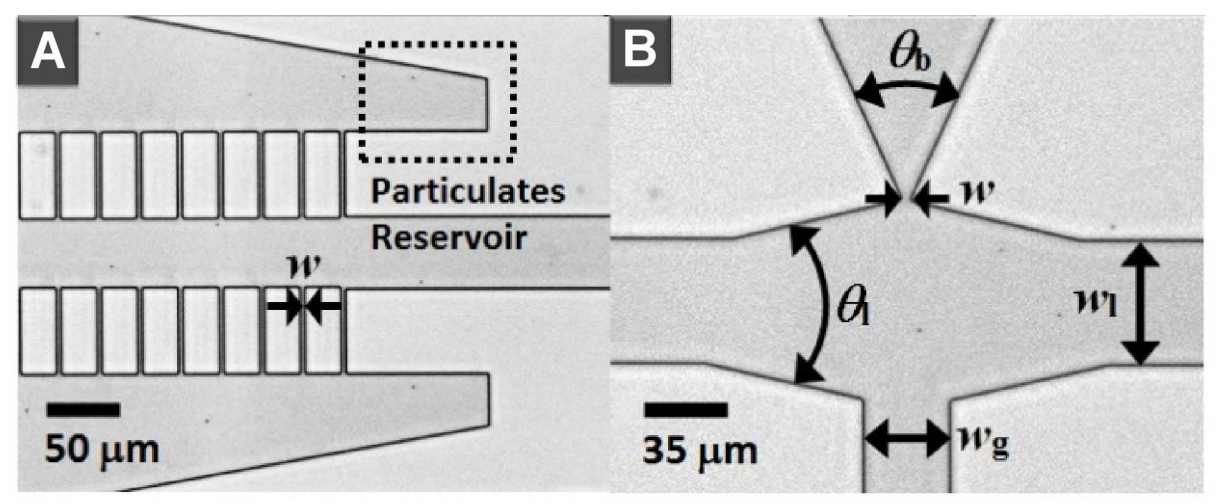

Figure 3.1: (A) Enlarged view of the filter and reservoir for trapping particulates. Filter channels are set to the same size as the nozzle, $w$. (B) Enlarged view of the expanding nozzle. Flare angles for the liquid channel and expanding nozzle are $\theta_{l}$ and $\theta_{b}$ respectively. The channel width for the liquid and gas channel are $w_{l}$ and $w_{g}$, respectively.

\subsection{Material and Methods}

\subsubsection{Microfluidic device fabrication}

Soft lithography techniques described in section 2.3.1 were used to fabricate the 2-D expandingnozzle FFMD. The dimensions of the specific design (Fig. 2.2c, Fig. 3.1) used for this study are listed in Table 3.1. To simplify optical observation of the microbubbles, the PDMS layer was plasma bonded directly to glass slides ( $25 \mathrm{~W}, 30 \mathrm{~s})$. After bonding, the device was immersed in deionized (DI) water to maintain hydrophilicity of the channels [41]. Again, to simplify production, PTFE tubing was directly inserted into the microfluidic device and sealed using two-part epoxy. To maintain a constant pressure drop across all three inlets all tubing was $15 \mathrm{~cm}$ long with a $0.76 \mathrm{~mm}$ outer diameter.

\subsubsection{Microbubble production}

Microbubbles were produced as described in section 2.3.2 with the exception that the liquid phase consisted of 2\% (v/v) Tween 20 surfactant (Sigma Aldrich St. Louis MO) [48, 49, 112] in deionized water. To characterize microbubble production, the gas pressure was held constant while the liquid flow rate was stepped in $0.5 \mu \mathrm{L} /$ min increments until the FFMD no 
Table 3.1: Microfluidic device dimensions

\begin{tabular}{ccc}
\hline Symbol & Parameter & Value \\
\hline \hline$\theta_{b}$ & Nozzle flare angle & $50^{\circ}$ \\
$\theta_{l}$ & Liquid flare angle & $25^{\circ}$ \\
$w_{g}$ & Gas channel width - Design & $35 \mu \mathrm{m}$ \\
& Measured & $37.1 \mu \mathrm{m}$ \\
$w_{l}$ & Liquid channel width - Design & $50 \mu \mathrm{m}$ \\
& Measured & $52.1 \mu \mathrm{m}$ \\
$w$ & Nozzle width - Design & $1 \mu \mathrm{m}$ \\
& Measured & $5.4 \mu \mathrm{m}$ \\
$h$ & Channel height - measured & $20.3 \mu \mathrm{m}$ \\
& Support post & $160 \mu \mathrm{m}$ \\
\hline
\end{tabular}

Non-measured parameters are the sizes specified on the mask. Support post parameter is diameter. The filter widths were designed on the mask to be the same size as the nozzle width, hence the reuse of $w$ in Fig. 3.1.

longer produced microbubbles. The FFMD was allowed to equilibrate for 5 min after any change in gas pressure or liquid flow rate. Microbubble diameter and production rate were measured optically via a microscope connected to a high speed framing camera as described in section 2.3.2. The device was washed and stored in deionized water after each experiment to maintain the hydrophilicity of the PDMS channel surface. Repeatability of experiments was tested by repeating diameter and production measurements over several days using the same input parameters.

\subsubsection{Filter characterization}

To determine the smallest particulate that could be trapped by the filter, uniform microspheres (Polysciences, Inc., Warrington, PA) of increasing size were flowed through the inlets. The effect of filter blockage on microbubble production was characterized by blocking an increasing percentage of filter channels with the microspheres prior to microbubble production. Microbubble diameter and production rate were then measured and compared at several gas pressure and liquid flow rate combinations. 


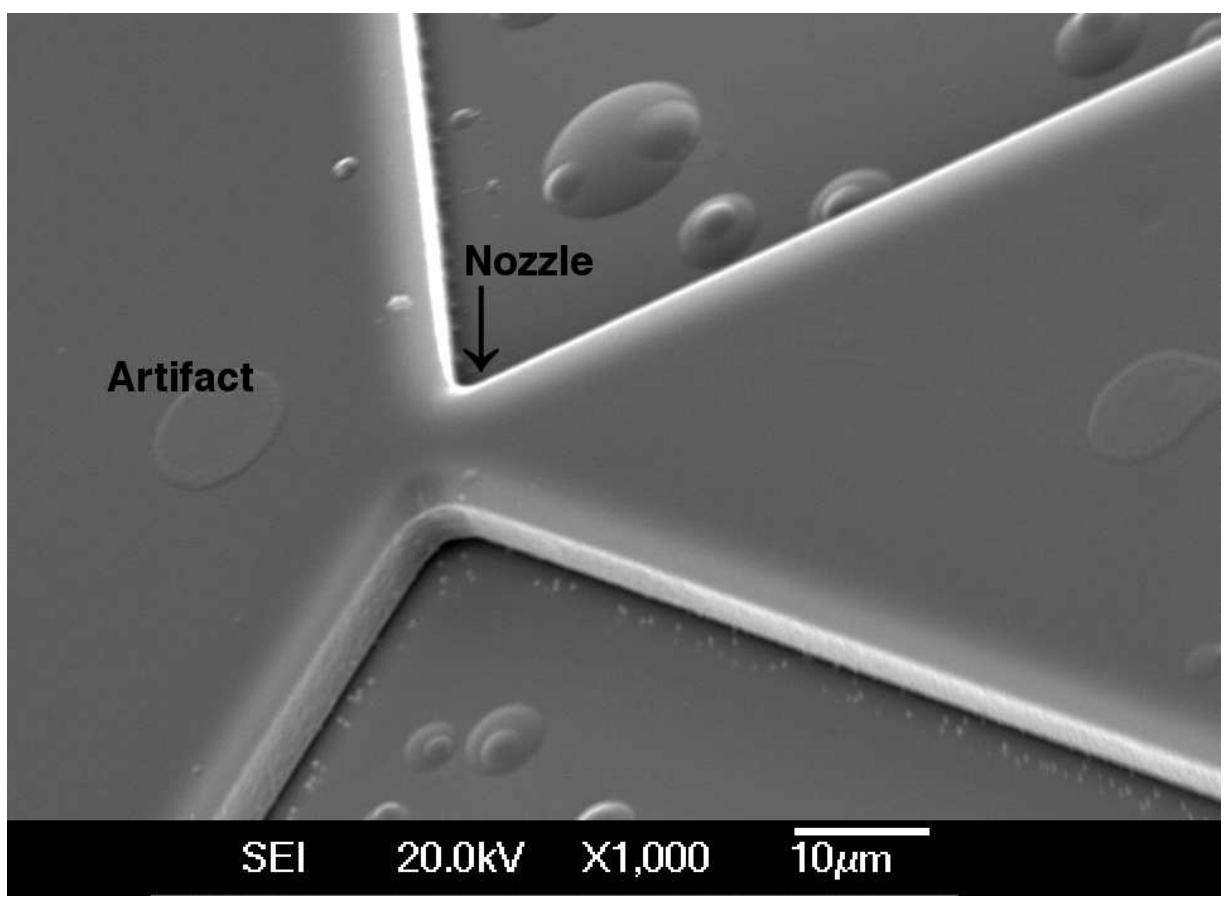

Figure 3.2: Scanning electron microscopy image of the final SU-8 mold demonstrating vertical side wall. Circular objects are artifacts of the sputtering process necessary for sample imaging.

Table 3.2: Flow conditions and experimental results when operating in the stable regime (III)

\begin{tabular}{cc}
\hline Property & Value \\
\hline \hline Liquid viscosity of Tween solution $(\mathrm{mPa})[113,114]$ & 0.9 \\
Surface tension of Tween solution $(\mathrm{mN} / \mathrm{m})[111,114]$ & 39.5 \\
Gas pressure range $(\mathrm{kPa})$ & $20.7-45.5$ \\
Liquid flow rate range $(\mu \mathrm{L} / \mathrm{min})$ & $32.0-64.0$ \\
MB diameter range $(\mu \mathrm{m})$ & $20.5-4.5$ \\
MB production rate $(\mathrm{MB} / \mathrm{s})$ range & $(0.022-4) \times 10^{5}$ \\
\hline
\end{tabular}

\subsection{Results and Discussion}

\subsubsection{Device fabrication}

Scanning electron microscope images of SU-8 molds indicated that the photoresist was fully developed, and all the structures had vertical sidewalls (Fig. 3.2). The measured nozzle width $(w)$ of the final PDMS cast FFMDs was $5.4 \mu \mathrm{m}$ when using $1.0 \mu \mathrm{m}$-wide nozzle molds. The width of liquid and gas channels $\left(w_{l}, w_{g}\right.$ respectively) were $2.1 \mu \mathrm{m}$ wider than the mask (Table 3.1). Channel height $(h)$ was $20.3 \mu \mathrm{m}$. 


\subsubsection{Flow conditions and experimental results}

Liquid properties, gas pressure, and liquid flow rate over which the FFMD was stably operated are listed in Table 3.2. When testing the filters, it was observed that microspheres less than $3 \mu \mathrm{m}$ in diameter passed freely through the filters, while particles greater than $10 \mu \mathrm{m}$ were completely trapped by the filter. Diameters in between were partially trapped by the filter (e.g. $50 \%$ of $6 \mu \mathrm{m}$ beads were trapped). The production rate and diameter of microbubbles produced with an FFMD with up to $90 \%$ of filters blocked by $10 \mu$ m diameter beads changed by less than $13 \%$ and $3 \%$, respectively.

The smallest microbubbles were produced at the highest production rate $-2.9 \mu \mathrm{m}$ diameter at $2 \times 10^{6}$ microbubbles/second $(\mathrm{MB} / \mathrm{s})$ - when the FFMD was operating at the highest gas pressure and liquid flow rate, $103 \mathrm{kPa}$ and $117 \mu \mathrm{L} / \mathrm{min}$ respectively. However, the FFMD was unstable under these parameters, and production could not be maintained for more than one minute. One hypothesis is that at these high pressures and flow rates, random fluctuations in the input parameters (e.g. tube motion, jitter in the syringe pump) might cause the liquid phase to push the gas cone away from the nozzle and stop microbubble production. Once production has stopped, random flow rate fluctuations are not enough to restore production.

\subsubsection{Microbubble Generation Regimes}

Microbubble production from the expanding-nozzle FFMD was divided into five regimes (Table 3.3, Fig. 3.3, and Fig.2.4). The FFMD transitioned sequentially through these production regimes when operated at a fixed gas pressure and increasing liquid flow rate [41, 100, 119]. The first and last regime produced no microbubbles due to over-pressure by the gas and liquid, respectively. Microbubble production occurred over the middle three regimes. In the first (Regime II), the microbubble diameter was larger than the height of the channel resulting in cylindrical microbubbles. In the second (Regime III), the microbubble diameter was less than the channel height and microbubble production was observed to be most stable. 


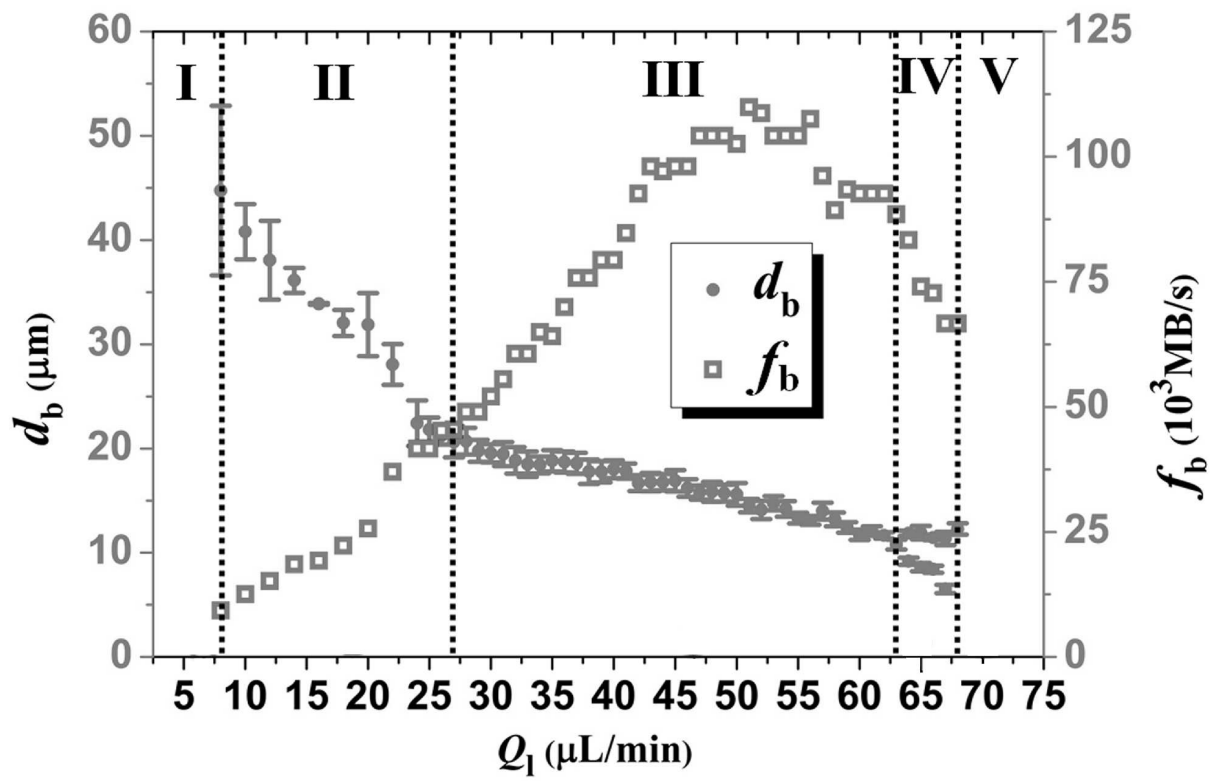

Figure 3.3: $\mathrm{MB}$ diameter and production rate curves for a fixed gas pressure $(41.4 \mathrm{kPa})$, and increasing liquid flow rate. Roman numerals denote regimes as described in Table 3.3 and Fig. 2.4. Scale bar $=20 \mu \mathrm{m}$ for all images.

In the last (Regime IV), multiple microbubbles formed during each pinch-off event. For all liquid flow rates and gas pressure combinations, peak microbubble production occurred within the stable regime (Regime III). In addition, the microbubbles with the smallest diameter were always produced at the transition between the stable (Regime III) and unstable regime (Regime IV). As microbubbles were most reliably produced within the stable regime (Regime III), the model was developed to predict production rate within this regime.

The stable regime (III) was further subdivided into geometry-controlled and flow-controlled regimes (Fig. 3.4). In the geometry-controlled regime, the narrowest part of the gas cone was physically restricted by the dimensions of the nozzle. When generating a microbubble in this regime, the tip of the gas cone appeared to block the nozzle completely in the width dimension. As a result, the microbubble formed downstream of the nozzle as the upstream neck of the gas thread was squeezed inwards by the liquid phase. After microbubble pinch-off, the tip of gas cone would retract upstream of the nozzle [111]. The gas cone tip would then move back towards the nozzle prior to penetrating and repeating the microbubble pinch-off process. The geometry-controlled regime was observed at lower gas pressures $(P \leq 41.3 \mathrm{kPa})$ 
Table 3.3: Production regimes at fixed gas pressure

\begin{tabular}{lll}
\hline Name & Characteristics & Liquid flow rate $(\mu \mathrm{L} / \mathrm{min})$ \\
\hline \hline Regime I & $\begin{array}{l}\text { No microbubbles (MB), gas streams continuously } \\
\text { through nozzle }[51]\end{array}$ & {$[0,8)$} \\
$\begin{array}{l}\text { Regime II } \\
\text { (cylindrical } \\
\text { regime) }\end{array}$ & $\begin{array}{l}\text { MB diameter larger than the height of the channel } \\
{[51,48]}\end{array}$ & {$[8,27)$} \\
$\begin{array}{l}\text { Regime III } \\
\text { (stable } \\
\text { regime) }\end{array}$ & MB diameter smaller than the height of the channel & {$[27,63)$} \\
$\begin{array}{l}\text { Regime IV } \\
\text { (unstable } \\
\text { regime) }\end{array}$ & $\begin{array}{l}\text { Each pinch-off event results in several microbubbles } \\
\text { of different size }[51,112]\end{array}$ & {$[63,68)$} \\
Regime V & No MBs, liquid streams continuously through nozzle & {$[68, \infty)$} \\
\hline
\end{tabular}

Gas pressure was held constant at $41.4 \mathrm{kPa}$

and liquid flow rates $\left(Q_{l} \leq 58 \mu \mathrm{L} / \mathrm{min}\right)$. At high gas pressures $(P>41.3 \mathrm{kPa})$ and liquid flow rates $\left(Q_{l}>58 \mu \mathrm{L} / \mathrm{min}\right)$ [52], the flow-controlled regime was observed. In this regime, the tip of the gas cone was less than the width of the nozzle, and stayed downstream of the nozzle. During both pre- and post- microbubble pinch-off, the tip remained downstream of the nozzle. Due to limits in optical resolution, microbubble events within the flow-controlled regime could not be measured. As a result, the production model was focused on the geometry-controlled regime.

\subsubsection{Microbubble generation modes}

Given the observed behavior of the gas cone, the microbubble generation cycle was separated into two parts: "bubbling time" $\left(\tau_{b}\right)$ and "waiting time" $\left(\tau_{w}\right)$. Bubbling time starts when the tip of the gas cone enters the nozzle and ends with microbubble pinch-off (Fig. 3.4, top $0.0-$ $6.3 \mu \mathrm{s})$. Waiting time starts at microbubble pinch-off and ends when the gas cone tip re-enters the nozzle (Fig. 3.4, bottom $7.7-13.3 \mu \mathrm{s}$ ). Combining these characteristic times resulted in 


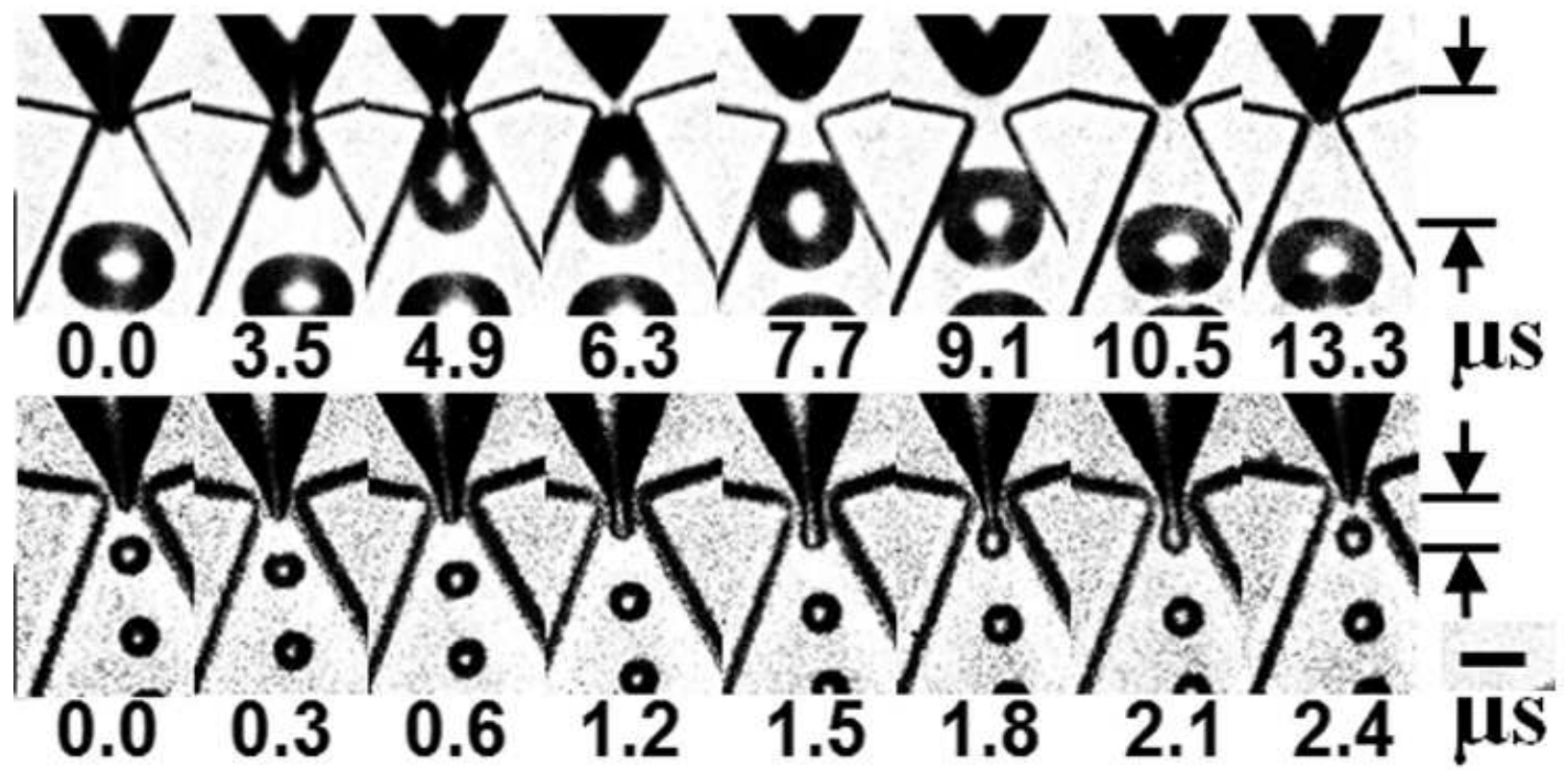

Figure 3.4: (Top) Microbubble pinch-off in the geometry-controlled regime. Within this production type subclassification, the tip of the gas cone oscillates over a larger extent (black arrows) and includes the nozzle. (Bottom) Flow-controlled regime. Within this regime, the tip of the gas cone oscillates over a shorter extent and stays downstream of the nozzle. Scale bar $=15 \mu \mathrm{m}$.

a relationship for microbubble production rate $\left(f_{b}\right)$ :

$$
f_{b}=\frac{1}{\tau_{b}+\tau_{w}}
$$

Consistent with Garstecki et al. [48, 111], $\tau_{b}$ was observed to decrease with increasing liquid flow rate, and was independent of gas pressure. Waiting time $\left(\tau_{w}\right)$ was observed to depend on both liquid and gas input parameters. At low gas pressures $(P \leq 37.9 \mathrm{kPa}), \tau_{w}$ increased with increasing liquid flow rate and decreased with increasing gas pressure. At higher gas pressures $(P \geq 41.4 \mathrm{kPa}), \tau_{w}$ decreased when either liquid flow rate or gas pressure increased.

\subsubsection{Microbubble production rate}

The bubbling time and waiting time were modeled after Garstecki et al. [48, 111] (eqn. 3.2). This relationship was extended by introducing the parameters $\beta$ and $\varepsilon$ to account for any 
dependence of these characteristic times on gas pressure:

$$
\begin{gathered}
\tau_{b}=\frac{\beta_{b}(P)}{Q_{l}}+\varepsilon_{b}(P), \\
\tau_{w}=\frac{\beta_{w}(P)}{Q_{l}}+\varepsilon_{w}(P),
\end{gathered}
$$

where the units of $\tau$ and $Q_{l}$ are $\mu$ s and $\mu \mathrm{L} / \mathrm{min}$ respectively, and $\beta$, and $\varepsilon$, were empirically determined. High speed images of geometry-controlled microbubble production were used to calculate the waiting and bubbling times, which were then used to fit (eqn. 3.4) and (eqn. 3.5).

As noted previously, $\tau_{b}$ was observed to be linearly related to the reciprocal of liquid flow rate but independent of gas pressure across all flow rates and pressures tested $-R^{2}=0.88$. $\tau_{w}$ was also related to the inverse of liquid flow rate for a fixed gas pressure, but the slope $\left(\beta_{w}\right)$ and intercept $\left(\varepsilon_{w}\right)$ of this relationship was observed to be linearly related to gas pressure $-R^{2}=0.86$ and 0.92 , respectively:

$$
\begin{aligned}
\beta_{b}(P) & =\beta_{b}=477 \\
\varepsilon_{b}(P) & =\varepsilon_{b}=-6.25 \\
\tau_{b} & =\frac{477}{Q_{l}}-6.25 \\
\beta_{w}(P) & =105 P+4.43 \times 10^{3} \\
\varepsilon_{w}(P) & =-3.43 P+150 \\
\tau_{w} & =\frac{105 P+4.43 \times 10^{3}}{Q_{l}}-(3.43 P+150),
\end{aligned}
$$


where the unit of $P$ is $\mathrm{kPa}$. Combining (eqn. 3.5) and (eqn. 3.4) with (eqn, 3.3) results in a final expression for production rate as a function of gas pressure and liquid flow rate:

$$
f_{b}=\frac{Q_{l}}{\beta_{b}+\beta_{w}(P)+\left[\varepsilon_{b}+\varepsilon_{w}(P)\right] Q_{l}}
$$

where the units of $f_{b}, Q_{l}$ and $P$ are $10^{3} \mathrm{MB} / \mathrm{s}, \mu \mathrm{L} / \mathrm{min}$ and $\mathrm{kPa}$ respectively.

Within the gas pressure range $(37.9 \mathrm{kPa}<P<43.4 \mathrm{kPa})$, the model of production rate accurately matched the measured production rate $\left(R^{2}=0.91\right)$. In addition, $89 \%$ of the production rates predicted from the input gas and liquid parameters were within $15 \%$ of the actual measured production rate (Fig. 3.5). Interestingly, at both the highest pressure, (Fig. 3.5, filled markers) and the lowest pressure (Fig. 3.5, dotted box) tested, the model over-estimated production rate. As expected the model unreliably predicted the production rate of microbubbles produced in the flow-controlled regime (Fig. 3.5, square markers).

\subsubsection{Limitations}

For the production rate relationship, (eqn. 3.8), only a single channel geometry and nozzle width was investigated. Although the effect of nozzle diameter on production rate may be implicitly incorporated into $\beta$, $\varepsilon$, additional experiments are necessary to determine the effect of channel geometry on these relationships. Furthermore, the effect of viscosity on these parameters was not determined and will need further investigation.

In addition, (eqn. 3.8) is only valid for FFMD production of microbubbles within the geometry-controlled regime. Relationships describing the transition between regimes will need to be investigated as they are important in determining the gas pressure and liquid flow rates over which the model would be valid. Furthermore, we observed that the minimum microbubble diameter produced by an FFMD operating in the geometry-controlled regime was equal to the nozzle width. To produce smaller microbubbles, the FFMD had to transition into a flow-controlled regime. 


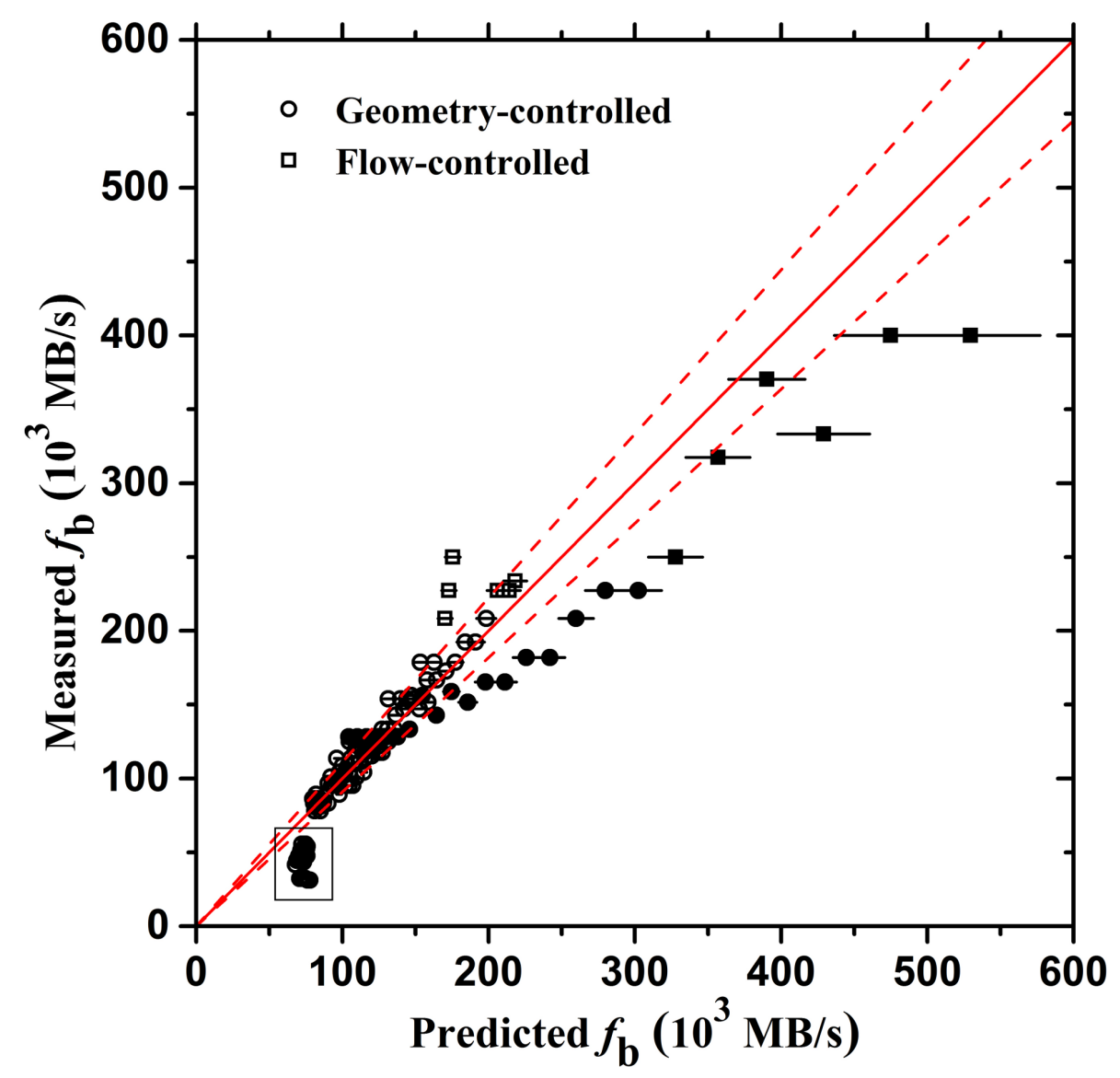

Figure 3.5: Comparison of measured microbubble production rate versus predicted production rate across all input parameters. Circle markers indicate geometry controlled and square markers indicate flow-controlled MB production, respectively. The dotted box outlines microbubble production rates measured at pressures between 29 and $34.5 \mathrm{kPa}$. At pressures between 37.9 and $43.4 \mathrm{kPa}, \mathrm{R}^{2}=0.91$ and $89 \%$ of the predicted values were within $15 \%$ of the measured values. At the highest pressure tested ( $P=45.5 \mathrm{kPa}$, filled markers), the model overestimated production rate.

We do not, however, expect channel height to significantly impact microbubble production rate. Changes in channel height would affect both the gas pressure and the liquid flow rate. Since microbubble production rate is related to the ratio of gas pressure and liquid flow, these changes should cancel. Previous work by Dietrich et al. [120] also suggests that the angle of the liquid channel intersection with the gas channel has a negligible effect. 


\subsection{Conclusion}

In this chapter a model for microbubble production from an expanding-nozzle FFMD operating in a geometry-controlled production mode was developed. In developing this model, microbubble production was observed to transition through five distinct regimes. Within the stable regime, microbubble pinch-off was observed to be either geometry-controlled or flow-controlled. Within the geometry-controlled regime, FFMD production was observed to transition between an additional two generation modes in which production rate was either independent or dependent of liquid flow rate. Finally, by analyzing two characteristic times bubbling time and waiting time - of microbubble pinch-off, a model for production rate was developed. 


\title{
Chapter 4
}

\section{Investigation of albumin stabilization}

\author{
for the production of
}

transiently-stable microbubbles from

a flow-focusing microfluidic device ${ }^{1}$

\subsection{Abstract}

In this chapter, I present a method of synthesizing albumin-shelled, large diameter $(>10 \mu \mathrm{m})$, transiently-stable microbubbles using a flow-focusing microfluidic device (FFMD). Microfluidic device production enables microbubbles to be produced immediately prior to utilization, thus relaxing the requirements for stability. Both reconstituted fractionated bovine serum albumin (BSA) and fresh bovine blood plasma were investigated as shell stabilizers. Microbubble coalescence was inhibited by the addition of either dextrose or glycerol and propylene glycol. Microbubbles were observed to have an acoustic half-life of approximately $6 \mathrm{~s}$. Microbubbles

\footnotetext{
${ }^{1}$ This chapter appears in the peer-reviewed publication:

J. L. Chen, A. H. Dhanaliwala, A. J. Dixon, A. L. Klibanov, and J. A. Hossack, "Synthesis and Characterization of Transiently Stable Albumin-Coated Microbubbles via a Flow-Focusing Microfluidic Device," Ultrasound Med Biol, vol. 40, no. 2, pp. 400-409, Feb. 2014.
} 
generated directly within a vessel phantom containing flowing blood produced a contrast to noise ratio of $15 \mathrm{~dB}$ when imaged with a special contrast pulse mode. These results demonstrate that transiently-stable microbubbles produced via flow-focusing microfluidic devices are capable of image enhancement. In addition, successful microbubble production with blood plasma suggests the potential to utilize blood directly as a stabilizing shell.

\subsection{Background}

Size distribution and stability are two important considerations for any microbubble formulation. As described in section 1.2 of the introduction, microbubble size is important as it dictates the acoustic properties but is also a source of risk as microbubbles exceeding $10 \mu \mathrm{m}$ in diameter pose an emboli risk [20, 17]. Stability is also important as it determines how well microbubbles survive the rigors of systemic circulation. Microbubbles can collapse during intravenous injection [81], they can be filtered by the lungs, liver and spleen [20, 80, 79], and they can dissolve in the complex multi-gas environment of the vasculature [103, 121]. To increase stability, newer generation microbubbles are formulated with low solubility gases and are stabilized with protein, polymer, or lipid shells [16] to reduce dissolution and increase circulation lifetime. For the in situ microbubble production paradigm, however, microbubbles with reduced stability were sought in order to enable the production of safe large diameter microbubbles.

Albumin is a plasma protein that has been extensively evaluated as a stabilizing shell material for microbubble contrast agents $[122,31,16]$. In order to form a stable shell, albumin is typically heated in order to denature and cross-link the protein [124]. Non-cross-linked albumin has a higher liquid-vapor surface tension (i.e. Laplace pressure) [125] and thus lower stability compared to cross-linked albumin-shelled microbubbles [124, 126]. Consequently, for in situ microbubble production, a non-cross linked albumin shell may be ideal as it could provide both biocompatibility and transient-stability. 
In this chapter, transiently-stable, albumin-coated, nitrogen microbubbles produced with an FFMD are characterized as a step towards accessing large microbubbles through the in situ microbubble paradigm. Both fractionated bovine serum albumin (BSA) and fresh bovine plasma were investigated. The produced microbubbles were characterized for diameter, production rate, and stability. Finally, acoustic contrast from in situ produced microbubbles in a flow phantom was measured.

\subsection{Materials and Methods}

\subsubsection{FFMD fabrication}

Flow-focusing microfluidic devices (FFMDs) were fabricated as described in section 2.3.1. Non-flooded microfluidic device was used to simplify fabrication as described in section 3.3.1. In addition, the devices were bound to PDMS, rather than glass, to improve device flexibility. The final, measured, dimensions of the gas and liquid channels were 35 and $50 \mu \mathrm{m}$ wide, respectively, the nozzle was $7 \mu \mathrm{m}$ wide, and all channels were $27 \mu \mathrm{m}$ tall.

\subsubsection{Microbubble fabrication}

The gas phase consisted of 99.998\% nitrogen (GTS Welco, Richmond, VA). The liquid phase consisted of 3 or $5 \%(\mathrm{w} / \mathrm{v})$ bovine serum albumin (BSA) dissolved in a solution of isotonic saline $(0.9 \% \mathrm{NaCl})$ or a solution of glycerol, propylene glycol, and isotonic saline (GPS). Dextrose was added to either solution as needed. All chemicals were purchased from Sigma-Aldrich (St. Louis, MO). Albumin concentration was determined by absorbance (NanoDrop 1000, Thermo Scientific, Wilmington, DE) assuming a molar extinction coefficient of $6.7 \mathrm{M}^{-1} \mathrm{~cm}^{-1}$ at $280 \mathrm{~nm}$. Viscosity was measured with an Ubbelohde viscometer (Cannon Instrument Company, State College, PA) at $23^{\circ} \mathrm{C}$. PTFE tubing (Cole Parmer Vernon Hills, IL) $30 \mathrm{~cm}$ in length was used to convey the liquid and gas phase to the microfluidic device inlets. Microbubbles were produced as described in section 2.3.2. 


\subsubsection{Microbubble characterization}

Microbubbles were characterized for production rate, diameter, and coalescence by analyzing images at the FFMD orifice and $1.7 \mathrm{~mm}$ downstream of the orifice using the imaging setup described in section 2.3.2.

Microbubble stability was estimated acoustically. A temperature controlled $\left(16,23,37^{\circ} \mathrm{C}\right)$ solution of deionized (DI) water and microbubbles was continuously mixed and imaged with a Siemens Sequoia 512 clinical scanner (15L8 transducer, Siemens Medical Solutions, Malvern, PA). The microbubbles were imaged with a contrast agent specific nonlinear imaging mode (Contrast Pulse Sequence [CPS] [99]) at a center frequency of $7 \mathrm{MHz}$ and mechanical index (MI) of 0.2 to maximize signal while minimizing microbubble destruction. Image gain was set at $0 \mathrm{~dB}$ to avoid signal saturation. An FFMD producing microbubbles was placed in the beaker until the ultrasound image intensity was constant. The FFMD was then removed and 8-bit B-mode images were acquired as described in section 2.4.3 for at least $60 \mathrm{~s}$. Intensity over time curves were calculated using a $30 \times 30$ pixel region of interest $(\mathrm{ROI})$ around the focus of the B-mode images ${ }^{2}$. Time-intensity curves were aligned by peak intensity and averaged. The microbubble acoustic half-life $\left(\mathrm{T}_{\mathrm{i}}\right)$ was calculated as the time until half the maximum intensity.

\subsubsection{Plasma microbubbles}

Bovine blood from an FDA approved abattoir was collected in $1.86 \mathrm{mg} / \mathrm{mL}$ ethylenediaminetetraacetic acid (EDTA). The plasma was separated from the blood by centrifugation at 500g for $30 \mathrm{~min}$ at $20^{\circ} \mathrm{C}$ to obtain a clear orange/pink supernatant. Large aggregates were filtered out using 8 and $2.5 \mu \mathrm{m}$ pore-size filter paper (Grade 2, 5, Whatman, Maidstone, ME) followed by $0.45 \mu \mathrm{m}$ pore syringe filters (Fisher Scientific, Waltham, MA). The plasma solution was used within 7 days of collection and was supplied to the FFMD as described above.

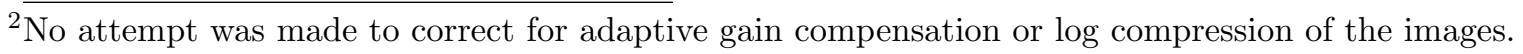


Plasma microbubbles were acoustically imaged under flow within a gelatin phantom, as described in section 2.3.3. The lumen diameter in the phantom was $4 \mathrm{~mm}$ to simulate imaging within a blood vessel. A FFMD producing $16 \mu$ m diameter plasma-stabilized microbubbles at a rate of $125,000 \mathrm{MB} / \mathrm{s}$ was placed in $100 \mathrm{~mL}$ of bovine blood under constant agitation. The blood and microbubbles were pulled through the flow phantom at a flow rate of $105 \mathrm{~mL} / \mathrm{min}$ (velocity $\approx 0.14 \mathrm{~m} / \mathrm{s}$ ) using a syringe pump. Microbubbles were imaged with the Sequoia $512(\mathrm{CPS}$ mode, center frequency $=7 \mathrm{MHz}, \mathrm{MI}=0.2)$ and signal improvement $(\mathrm{SI})$ was calculated as:

$$
S I=\mu_{w}-\mu_{w o}
$$

where $\mu_{w o}$ is the mean pixel intensity without microbubbles and $\mu_{w}$ is the mean pixel intensity with microbubbles. Signal improvement was measured directly from B-mode images that were acquired as described in section 2.4.3 and was measured within a $30 \times 30$ pixel window inside the lumen. Microbubble stability was measured in the flow phantom by stopping microfluidic production and flow, and measuring $T_{\mathrm{i}}$. Ultrasonic destruction of microbubbles was investigated by stopping flow, increasing the MI to 1.9, and measuring $\mathrm{T}_{\mathrm{i}}$.

\subsection{Results}

\subsubsection{Albumin microbubble production and coalesence}

Microbubbles with diameters between $10-20 \mu \mathrm{m}$ were successfully produced at rates between $1-6 \times 10^{5} \mathrm{MB} / \mathrm{s}$. At these high production rates, however, microbubbles stabilized with BSA alone were observed to coalesce downstream (Fig. 4.1a). At a constant concentration of $10 \%(\mathrm{w} / \mathrm{v})$ dextrose, BSA concentrations greater than $3 \%$ prevented coalescence (Fig. 4.1b). At a constant concentration of $3 \%$ BSA, dextrose concentrations greater than $5 \%$ eliminated coalescence. For GPS, however, only a concentration of $2.5 \%$ glycerol, $2.5 \%$ propylene glycol, 

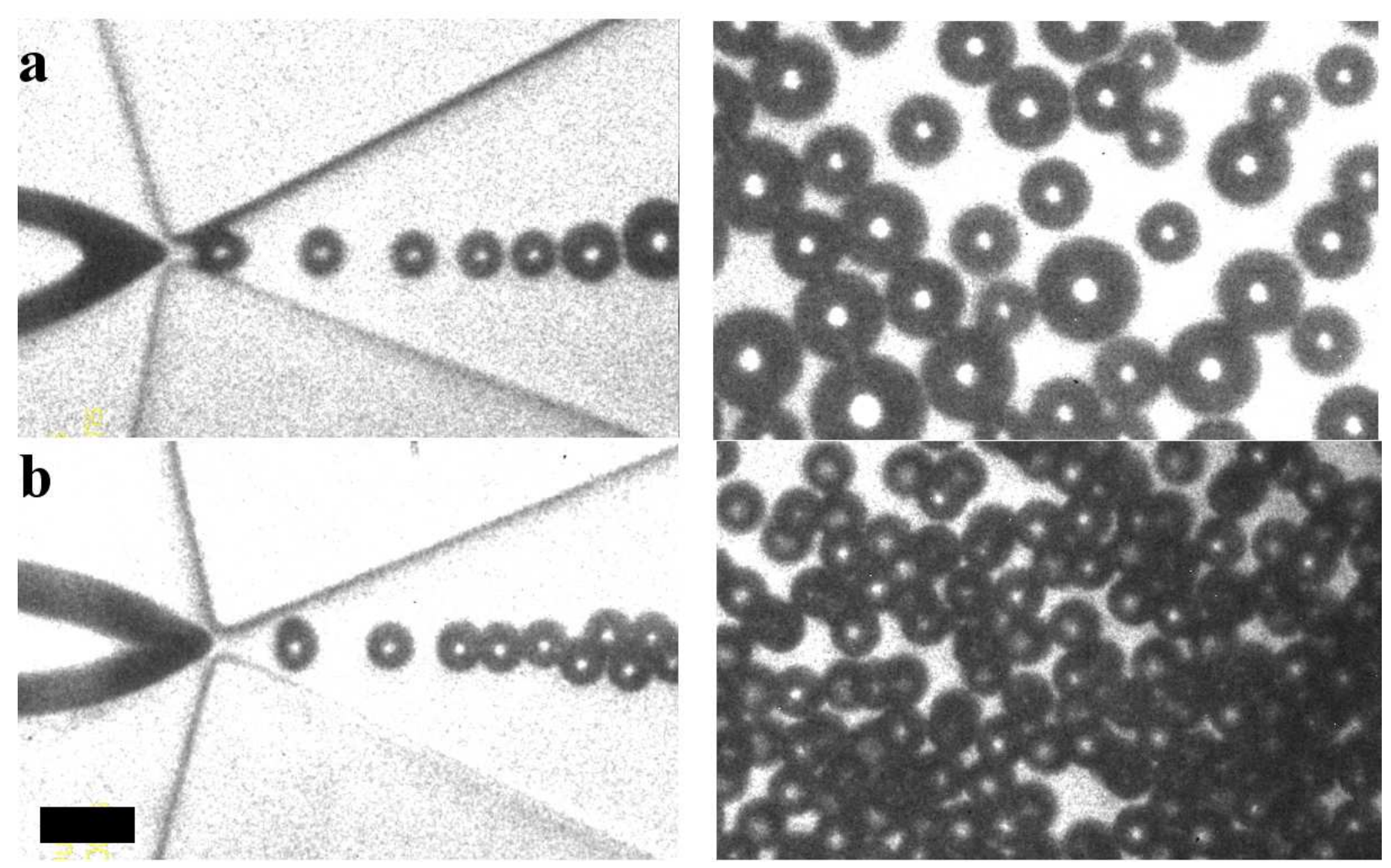

Figure 4.1: Microbubbles were imaged at both the FFMD nozzle and downstream of the nozzle. Representative high-speed images of (a) coalesced microbubbles and (b) stable microbubbles at the nozzle (left) and downstream (right) (scale bar $=20 \mu \mathrm{m}$ ). At a constant concentration of $10 \%$ dextrose increasing BSA prevented coalescence. At a constant concentration of $3 \%$ BSA increasing dextrose prevented coalescence.

and $95 \%(\mathrm{v} / \mathrm{v})$ saline $(2.5 \%$ GPS $)$ was observed to eliminate coalescence. A solution of only $10 \%$ dextrose in saline or $2.5 \%$ GPS both failed to produce stable microbubbles. A solution of $3 \%$ BSA had a viscosity of $1.10 \mathrm{mP}$ s. Adding $2.5 \%$ GPS increased the viscosity to $1.28 \mathrm{mP}$, while adding $10 \%$ dextrose increased the viscosity to $1.42 \mathrm{mP}$ s.

\subsubsection{BSA microbubble stability}

Microbubbles with $13 \mu \mathrm{m}$ diameters were used when measuring microbubble half-life. Microbubble half-life $\left(\mathrm{T}_{\mathrm{i}}\right)$ was not significantly different between microbubbles stabilized with $3 \%$ BSA and $10 \%$ dextrose (BSA-DEX) or 3\% BSA and 2.5\% GPS (BSA-GPS) at any temperature (Table 4.1 ). At $37^{\circ} \mathrm{C}$, microbubble half-life was $\approx 8$ s. Microbubble half-life 


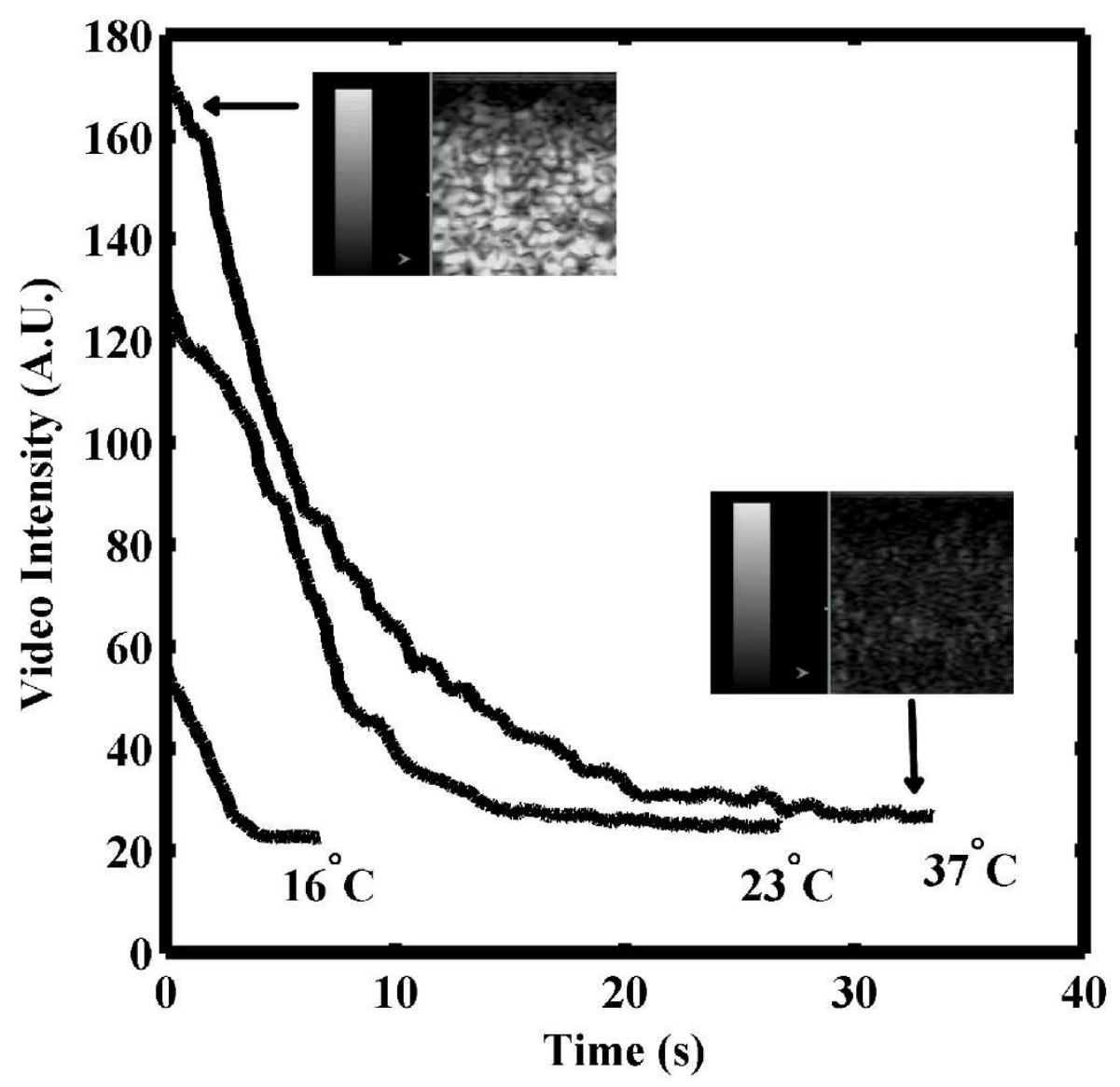

Figure 4.2: Representative image intensity curves used to calculate acoustic half-life Microbubbles stabilized with either BSA-GPS or BSA-DEX showed similar rates of signal loss over time. (Dynamic range scale $=50 \mathrm{~dB}$, and $\mathrm{CPS}$ gain $=0 \mathrm{~dB}$ ).

Table 4.1: Microbubble half-life

\begin{tabular}{ccc}
\hline & \multicolumn{3}{c}{ Half-life (s) } \\
Temperature $\left({ }^{\circ} \mathrm{C}\right)$ & $3 \%$ BSA, 10\% dextrose & $3 \%$ BSA and 2.5\% GPS \\
\hline \hline 16 & $3.7 \pm 1.5$ & $2.8 \pm 0.4$ \\
23 & $6.6 \pm 2.2$ & $6.8 \pm 1.9$ \\
37 & $8 \pm 3.6$ & $7.2 \pm 3.8$ \\
\hline
\end{tabular}

No significant difference in half-life between formulations at any given temperature. Microbubble half-life at $16^{\circ} \mathrm{C}$ was lower than microbubble half-life at $23^{\circ} \mathrm{C}$ and $37^{\circ} \mathrm{C}(\mathrm{p}<0.05, \mathrm{n}=5)$.

at $16^{\circ} \mathrm{C}$ was statistically different $(\mathrm{p}<0.05)$ from microbubble half-life at $23^{\circ} \mathrm{C}$ and $37^{\circ} \mathrm{C}$ (Fig. 4.2). 


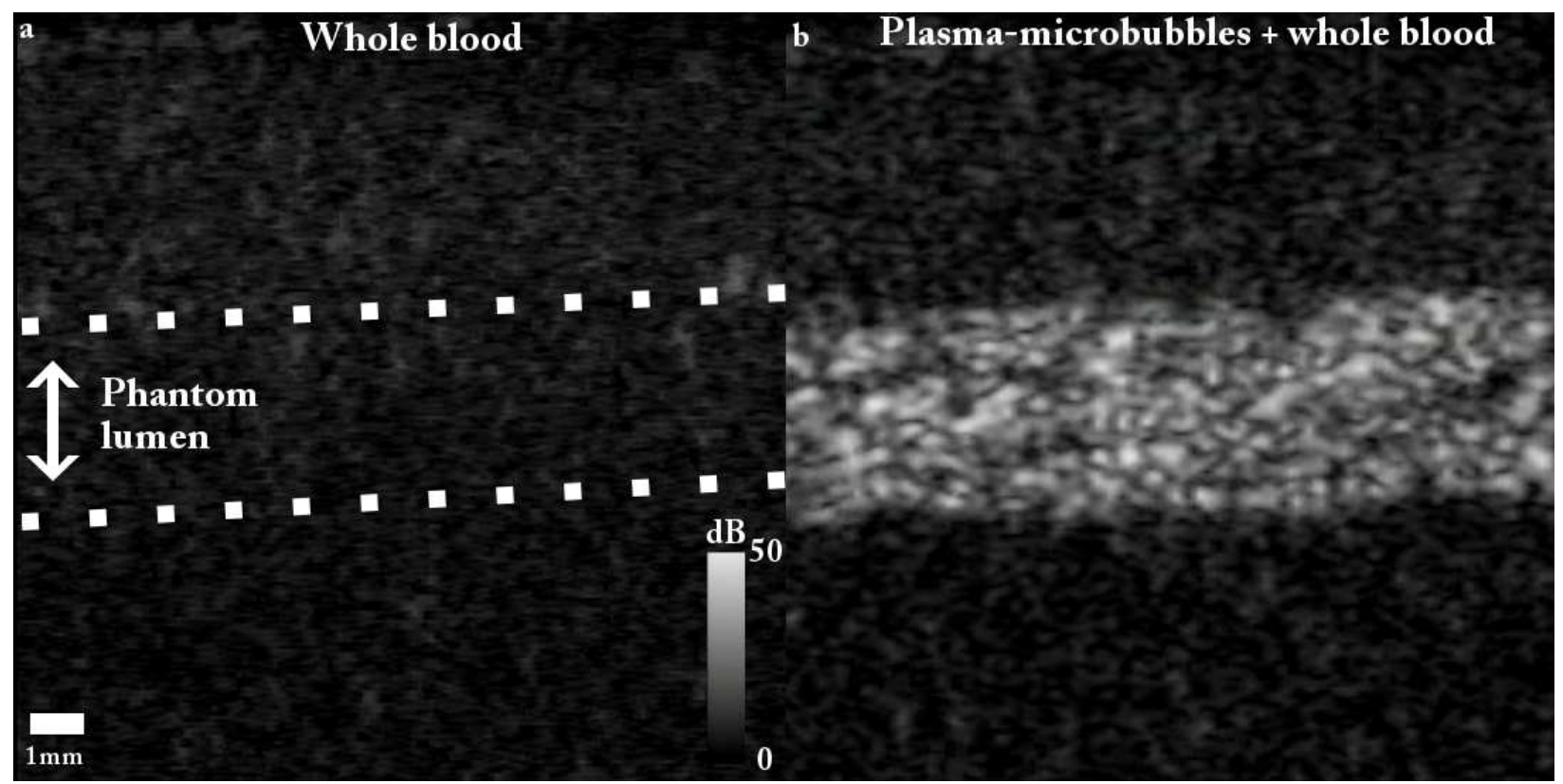

Figure 4.3: Gelatin phantom lumen (a) Pre- and (b) post- in situ microbubble production. Microbubbles imaged in whole blood using contrast imaging mode (CPS) resulted in an $\approx 102$-fold increase in image intensity within the lumen of the gelatin phantom. Acoustic half-life measured within the lumen with no flow was $3.2 \pm 0.2 \mathrm{~s}$. Increasing the MI to 1.9 caused complete microbubble destruction within $2 \mathrm{~s}$. Scale bar $=2 \mathrm{~mm}$, dynamic range scale $=50 \mathrm{~dB}$, CPS gain $=0 \mathrm{~dB}$ ).

\subsubsection{Bovine plasma microbubbles}

Similar to BSA-stabilized microbubbles, microbubbles stabilized with bovine plasma were produced with diameters between $10-16 \mu \mathrm{m}$ at rates on the order of $10^{5} \mathrm{MB} / \mathrm{s}$. Microbubbles stabilized with blood plasma alone quickly coalesced. Adding dextrose up to $40 \%$ reduced coalescence to less than $10 \%$, while adding a solution containing $2.5 \%$ glycerol and $2.5 \%$ propylene glycol eliminated coalescence.

Bovine plasma stabilized microbubbles produced in situ in whole blood and under flow resulted in an $\approx 102$-fold increase in image intensity within the gelatin phantom lumen when imaged in CPS mode (Fig. 4.3a). When flow was stopped, acoustic half-life at an MI $=0.2$ was measured to be $3.2 \pm 0.2 \mathrm{~s}$. Increasing the MI to 1.9, resulted in an acoustic half-life of $0.9 \pm 0.4 \mathrm{~s}$ (Fig. 4.3b) 


\subsection{Discussion}

\subsubsection{Microbubble coalescence and stability}

A solution of BSA with either GPS or dextrose prevented microbubble coalescence, and no significant difference in microbubble stability was found between formulations. Bulk liquid viscosity has been found to both prevent microbubble coalescence by increasing the coalescence time $[128,129]$ and facilitate coalescence by increasing the interaction between microbubbles [130]. Thus, these two opposing effects may explain the optimum GPS concentration $(2.5 \%)$ that was necessary to form stable albumin-coated microbubbles.

An acoustic metric was used to calculate microbubble half-life as it is more relevant to the signal that would be measured in vivo and it should capture both changes in microbubble size as well number. In addition, to better approximate the blood environment microbubble lifetimes were evaluated in air-saturated media. The decrease in microbubble half-life as a function of temperature is likely a consequence of the increased gas solubility that occurs with decreasing temperature.

Since neither heat nor sonication was used, it is unlikely that there was significant crosslinking between albumin molecules. Thus, weaker non-covalent forces (hydrogen bonds and hydrophobic interactions) may primarily be responsible for the formation of the shell [133]. Although rapid microbubble dissolution may not be ideal for systemic administration, it should satisfy the goal of minimizing the risk of gas emboli formation from large microbubbles.

\subsubsection{Plasma microbubbles}

The ability to use albumin to stabilize the microbubble suggests that it may be possible to using a patient's own blood to produce microbubbles. By using autologous albumin, the small risk of an immune response to the microbubble shell could be reduced, if not

eliminated. In addition, by obtaining plasma immediately prior to microbubble fabrication, there would be no need to store any materials. While ideally microbubbles would be stabilized 
by plasma alone, additional stabilizers had to be added to the plasma to prevent microbubble coalescence. Additional studies are needed to evaluate what effects other surface active components in plasma, such as proteins, clotting factors, lipids, and cholesterols, may have on microbubble stabilization or prevention thereof. Most significantly, we observed that plasma microbubbles produced at rates as low as $1 \times 10^{5} \mathrm{MB} / \mathrm{s}$ were capable of producing acoustic contrast (Fig. 4.3a). Traditionally, microbubbles are administered on the order of $10^{9}$ microbubbles per patient ([78]; [134]). By locally producing microbubbles, we were able to achieve contrast with only a few seconds of microbubble production. Additional studies are necessary to compare the acoustic signal from equivalent concentrations of current generation microbubbles and FFMD-produced microbubbles. However, these results suggest that in situ produced microbubbles may not need to achieve high microbubble production rates (i.e. > $\left.10^{6} \mathrm{MB} / \mathrm{s}\right)$ to generate sufficient acoustic contrast.

\subsubsection{Limitations}

The focus of the present study was to investigate and characterize albumin-stabilized microbubbles produced via an FFMD. While the ultimate goal is to develop the paradigm of in situ microbubble production, we chose to use an FFMD with non-vascular compatible dimensions as these devices provide larger imaging windows through which the microbubbles can be optically characterized. Similarly, an FFMD placed at the tip of a catheter would require the gas input tubing to be greater than $130 \mathrm{~cm}$ in length in order to extend the length of the catheter. We chose to use shorter tubing in order to focus on microbubble characterization. Consequently, the effect of device size and input tubing length on FFMD operation and microbubble production requires additional study.

As our goal was only to verify that plasma-stabilized microbubbles could be successfully imaged within blood, we did not compare the acoustic signal with that from traditional albumin microbubbles such as Optison (GE Healthcare). Using an optimized and consistent 
set of imaging parameters will enable future studies to compare the acoustic signal from multiple microbubble contrast agent formulations across multiple imaging studies.

Because of inter-device variation with current fabrication methods, a specific set of gas and liquid input parameters does not always result in exactly the same microbubble diameter and production rate across multiple microfluidic devices. However, once an FFMD has been characterized and calibrated, the resulting microbubble diameter and production rate for a given set of gas and liquid input parameters were within $10 \%$ of their expected values. Furthermore, while we can optically confirm microbubble diameter and production rate before and after, we currently cannot directly confirm microbubble properties during an ultrasound imaging procedure.

\subsection{Conclusions}

In this chapter, I report on the stabilization of microfluidic produced microbubbles using BSA and blood plasma in order to achieve transient-stability. Microbubbles stabilized with albumin and plasma alone were observed to coalesce. An additional stabilizer, either dextrose or glycerol plus propylene glycol, prevented coalescence. Significantly, despite the short microbubble half-life, in situ produced microbubbles were capable of producing acoustic contrast. Overall, the ability to produce microbubbles stabilized with plasma is another step toward the goal of utilizing flow-focusing microfluidic devices for in situ production of transiently-stable microbubbles. 


\section{Chapter 5}

\section{Microfluidic-produced microbubbles}

\section{for enhanced drug delivery in vitro ${ }^{1}$}

\subsection{Abstract}

Microbubbles have been shown to enhance drug delivery in the presence of ultrasound. In this chapter, I present work evaluating whether transiently-stable microbubbles in combination with ultrasound are able to achieve a similar level of drug delivery enhancement. Using the in situ microbubble production paradigm, microbubbles can be produced in real-time and in close proximity to the therapeutic site. Rat aortic smooth muscle cells were placed in a flow chamber designed to simulate physiological flow conditions. A flow-focusing microfluidic device (FFMD) was placed upstream of the cells to produce microbubbles while ultrasound was applied to enhance uptake of a surrogate drug - the membrane impermeable fluorophore calcein. The microbubbles consisted of a nitrogen core and were stabilized with a polyethylene glycol-40 stearate shell. Calcein uptake was observed at acoustic pressures as low as $100 \mathrm{kPa}$.

${ }^{1}$ This chapter appears in the peer-reviewed publication:

A. J. Dixon, A. H. Dhanaliwala, J. L. Chen, and J. A. Hossack, "Enhanced Intracellular Delivery of a Model Drug Using Microbubbles Produced by a Microfluidic Device," Ultrasound Med Biol, vol. 39, no. 7, pp. 1267-1276, Jul. 2013. 
These results demonstrate that transiently-stable microbubbles retain the capability to enhance drug delivery in combination with ultrasound.

\subsection{Background}

Numerous reports studying the effect of various ultrasound (e.g. frequency, pressure, duty cycle) and microbubble (e.g. shell composition, microbubble concentration) parameters on drug and gene delivery have been published [140, 141, 142, 143, 144, 145]. Although there is increasing interest in ultrasound-mediated drug and gene delivery the effects of blood flow on ultrasound-mediated delivery have not been extensively studied. The majority of parameter optimization has been conducted in vitro under static conditions. Yet, it is known that blood flow patterns alter local drug and microbubble concentrations near the vessel wall [148]. Consequently, adding fluid flow across cells in in vitro models of drug delivery may allow for better translation of ultrasound parameters to an in vivo setting.

Enhanced delivery requires precise control over both ultrasound and microbubble parameters. While ultrasound parameters are easily controlled and models exist for the propagation of acoustic waves through tissue [149, 150], the properties of microbubbles are less predictable. As described in section 1.3 of the introduction, current microbubble production techniques utilize agitation methods [17] to generate stable microbubbles with a wide range of diameters. This wide range of diameters may affect drug delivery efficacy since the microbubble resonance frequency may not be matched to the insonation frequency. In addition, stable microbubbles must be less than $10 \mu \mathrm{m}$ in diameter to minimize emboli risk, even though larger microbubbles may provide improved drug delivery [37, 38]. Second, as described in section 2.2, a large percentage of microbubbles are lost following intravenous administration and systemic circulation resulting in poor control of microbubble and drug concentrations at the therapeutic target site. Finally, while the acoustic properties of single microbubbles have been experimentally observed and modeled [151, 152, 153, 154, 155], the interactions between 
clouds of oscillating microbubbles and the impact of these interactions on the processes that govern drug delivery remain unclear.

In situ microbubble production may overcome many of these limitations by enabling controlled and local administration of a known number of microbubbles and amount of drug. As described in section 2.2, flow-focusing microfluidic devices (FFMDs) are ideal for this application, as they have a small footprint and can produce microbubbles in real-time, thus allowing the microbubbles to be "tuned" to the requirements of specific applications. Producing microbubbles directly within the vasculature may significantly reduce the number of microbubbles needed for contrast enhancement or drug delivery, as losses due to storage, administration, and circulation are reduced or eliminated. In addition, local production may enable more accurate estimates of microbubble concentration at the site of interest. The greatest advantage to drug delivery with the in situ production paradigm, however, may be the ability to utilize larger microbubbles, as evidence suggests that larger microbubbles may provide improved cell permeabilization for drug uptake $[156,137]$.

In this chapter, I investigate the feasibility of in situ produced microbubbles for drug delivery via an in vitro model. To better simulate in vivo flow conditions, drug delivery was performed on cells experiencing flow.

\subsection{Materials and Methods}

\subsubsection{FFMD fabrication}

Non-flooded flow-focusing microfluidic devices were fabricated as described in section 3.3.1. Final device dimensions were: channel height of $25 \mu \mathrm{m}$, nozzle width of $8 \mu \mathrm{m}$, and liquid and gas channel widths of 50 and $30 \mu \mathrm{m}$ wide, respectively. The overall dimension of the device used in this study was $10 \times 4 \times 8 \mathrm{~mm}$. Devices were stored in deionized water to maintain the hydrophilicity of the channels and were used within five days. 


\subsubsection{Microbubble characterization}

Microbubbles were fabricated and characterized as described in section 2.3.2. The gas phase was $99.998 \%$ pure nitrogen (GTS Welco, Richmond, VA). The liquid phase consisted of $15 \mathrm{mg} / \mathrm{mL}$ polyethylene glycol-40-stearate (PEG40St) dissolved in a solution of $10 \%$ glycerol, $10 \%$ propylene glycol, and $80 \%$ phosphate buffered saline with magnesium and calcium (v/v) (GPS+). All chemicals were purchased from Sigma-Aldrich (St. Louis, MO). The PEG40StGPS+ solution was sonicated to $60^{\circ} \mathrm{C}$ with a tip sonicator (XL2020,Misonix, Farmingdale, NY) using a half-inch probe (40\% power, $7 \mathrm{~min})$. Immediately prior to use, the solution was sterile filtered (0.45 $\mu \mathrm{m}$ nylon filter, Fisher Scientific Waltham, MA).

To determine microbubble stability, a FFMD producing microbubbles was placed in $100 \mathrm{~mL}$ of air-saturated saline for 5 seconds at $25^{\circ} \mathrm{C}$. The FFMD was removed and the solution was then analyzed using a Coulter Particle Counter (Z2 particle analyzer, Beckman Coulter, Brea, CA). The solution was stirred continuously and measurements were taken every 30 seconds for 2 minutes.

\subsubsection{Cell culture and calcein delivery}

Primary rat aortic smooth muscle cells were used as a model cell for drug delivery. The cells were plated on Thermanox coverslips (Nunc, Rochester, NY) at a density of $5 \times 10^{3}$ cells $/ \mathrm{cm}^{2}$ and incubated at $37^{\circ} \mathrm{C}$ in a $5 \% \mathrm{CO}_{2}$ environment. The smooth muscle cells were cultured in growth media (DMEM/F12 plus 10\% fetal bovine serum, Gibco, Grand Island, NY) and were allowed to reach $100 \%$ confluency prior to experimentation.

A custom flow chamber (Fig. 5.1A) designed to achieve laminar flow was constructed from cast acrylic. The chamber was $0.2 \mathrm{~cm}$ high by $2.4 \mathrm{~cm}$ wide by $18 \mathrm{~cm}$ long and contained an acoustic window (50 $\mu \mathrm{m}$ thick mylar, McMaster-Carr, Atlanta, GA) half-way down its length. The dimensions were selected to ensure a Reynolds number (eqn. C.1), assuming a characteristic length of a rectangular channel (eqn. C.4), less than 2000 [158] for all flow rates. The shear stress experienced by the cells was calculated from (eqn. C.5). The cells were placed 

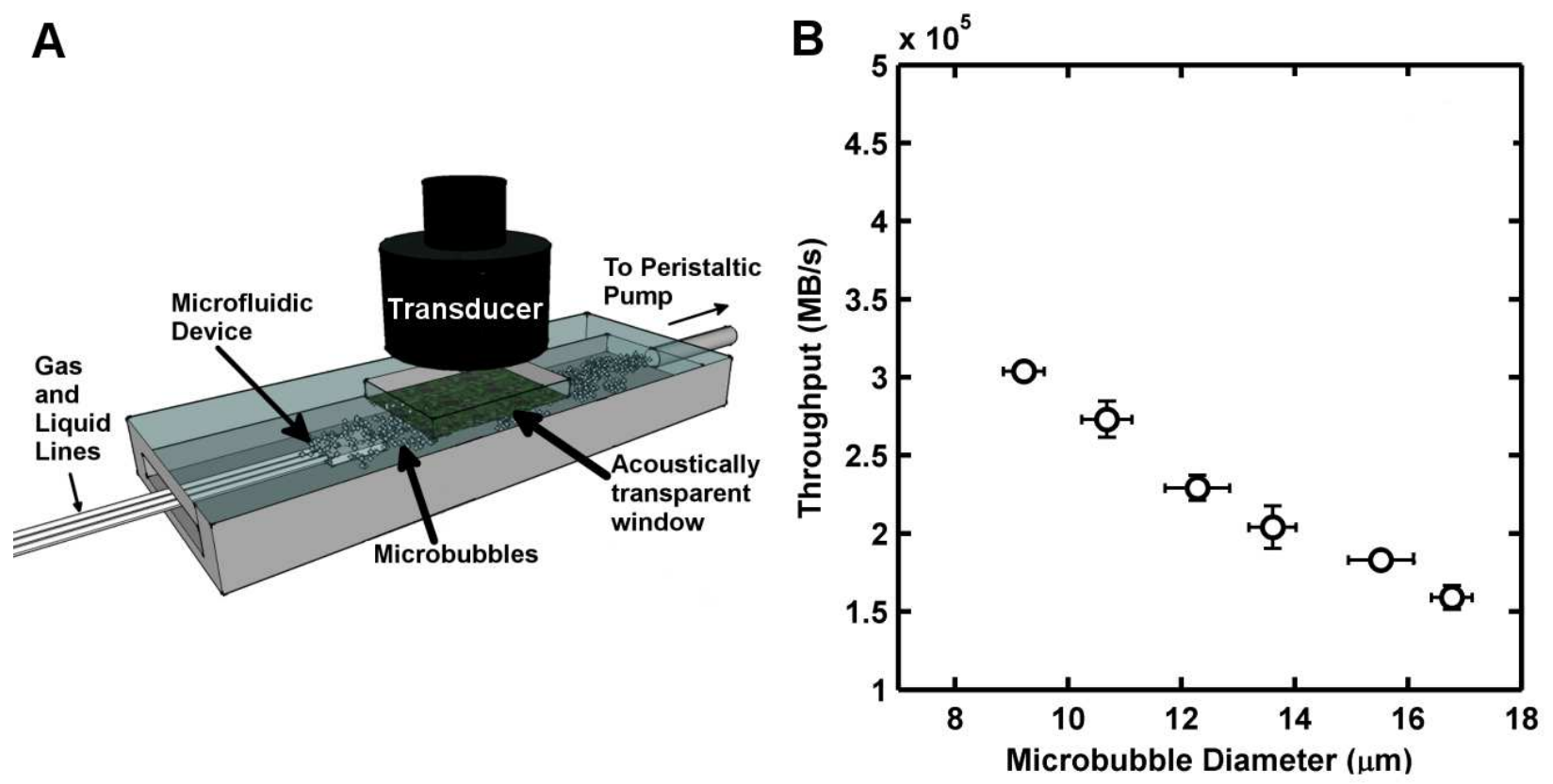

Figure 5.1: (A) Schematic of the in vitro flow chamber. A flow-focusing microfluidic device produced microbubbles in situ. Calcein was administered alongside the microbubbles, and a single element ultrasound transducer insonated the microbubbles as they flowed by confluent rat aortic smooth muscle cells. (B) Microbubble production rate and diameter are coupled as liquid flow rate changes. Liquid flow rate decreases from left to right. Gas pressure was constant.

within the acoustic window ${ }^{2}$, and the entire flow chamber was submerged in a bath of $155 \mathrm{mM}$ phosphate buffered saline containing calcium and magnesium (PBS +/+) (Gibco, Grand Island, NY) at $37^{\circ} \mathrm{C}$. Flow was established by pulling PBS $+/+$ through the flow chamber using a peristaltic pump (Wheaton Science Products, Millville, NJ). Microbubbles were introduced by placing a pre-characterized FFMD upstream of the cells within the channel.

Calcein (Sigma-Aldrich, St. Louis, MO), a cell membrane impermeable fluorophore was used as a model therapeutic since only cells that are permeabilized should internalize calcein [140]. A concentrated calcein solution was administered from a separate tube affixed to the FFMD to achieve a final concentration of $0.05 \mathrm{mg} / \mathrm{ml}$ within the flow chamber.

Ultrasound was applied with a focused, $500 \mathrm{kHz}, 1$ " diameter single-element ultrasound transducer (V301 Olympus Panametrics, Waltham, MA). To achieve a wider insonation beam

\footnotetext{
${ }^{2}$ The acoustic window was not recessed, resulting in a small step due to the height of the Thermanox membranes $(70 \mu \mathrm{m})$. While this may produce small eddies at the edge of the membrane, it is unlikely to affect the flow experienced by the cells in the center of the membrane.
} 
width, the transducer was placed at half its focal distance above the acoustic window. The transducer was driven by a 30 cycle, $500 \mathrm{kHz}$ sinusoid with a pulse repetition frequency (PRF) of $100 \mathrm{~Hz}$. The waveform was produced with an arbitrary waveform generator (AWG3022B, Tektronix, Beaverton, OR) and amplified with a $60 \mathrm{~dB}$ RF power amplifier (A-500 ENI, Rochester, NY). Acoustic pressures were confirmed using a calibrated PVDF hydrophone (GL-0200, Onda Corp., Sunnyvale, CA). A center frequency of $500 \mathrm{kHz}$ was selected to match the resonance of the microbubbles [153].

The cells were insonated in the presence of microbubbles and calcein for a total of 2 minutes. The ultrasound and FFMD were then removed and the cells were incubated for an additional 2 minutes under flow to allow the cell membranes to reseal [156]. The Thermanox membranes containing the cells were then removed, stained for cell death with a $25 \mu \mathrm{g} / \mathrm{ml}$ solution of propidium iodide (PI) (MP Biomedicals, Santa Ana, CA), and finally imaged using fluorescence microscopy within 12 hours.

The effects of insonation pressure on delivery were tested at peak negative pressures (PNP) of 0, 100, 200, $300 \mathrm{kPa}$. The following controls were performed: (1) calcein delivery at $18 \mathrm{ml} / \mathrm{s}$ flow but without microbubbles or ultrasound, (2) calcein delivery without flow, without microbubbles, but with $300 \mathrm{kPa}$ PNP ultrasound, and (3) calcein delivery without flow, without ultrasound, but with FFMD generated microbubbles. In addition, calcein delivery was measured when calcein alone or calcein with the PEG40St-GPS+ used to make the microbubbles was flowed past the cells.

\subsubsection{Cell image analysis}

For each trial, four images within the central part of each membrane were acquired, and the number of cells that internalized calcein and the number of nuclei stained with PI in each image were counted. To account for cell dislodgement [160], percentages were calculated using the total number of cells prior to insonation. The total number of cells at $100 \%$ confluency for four membranes was less than $5 \%$ and was therefore assumed to be equal for all subsequent 


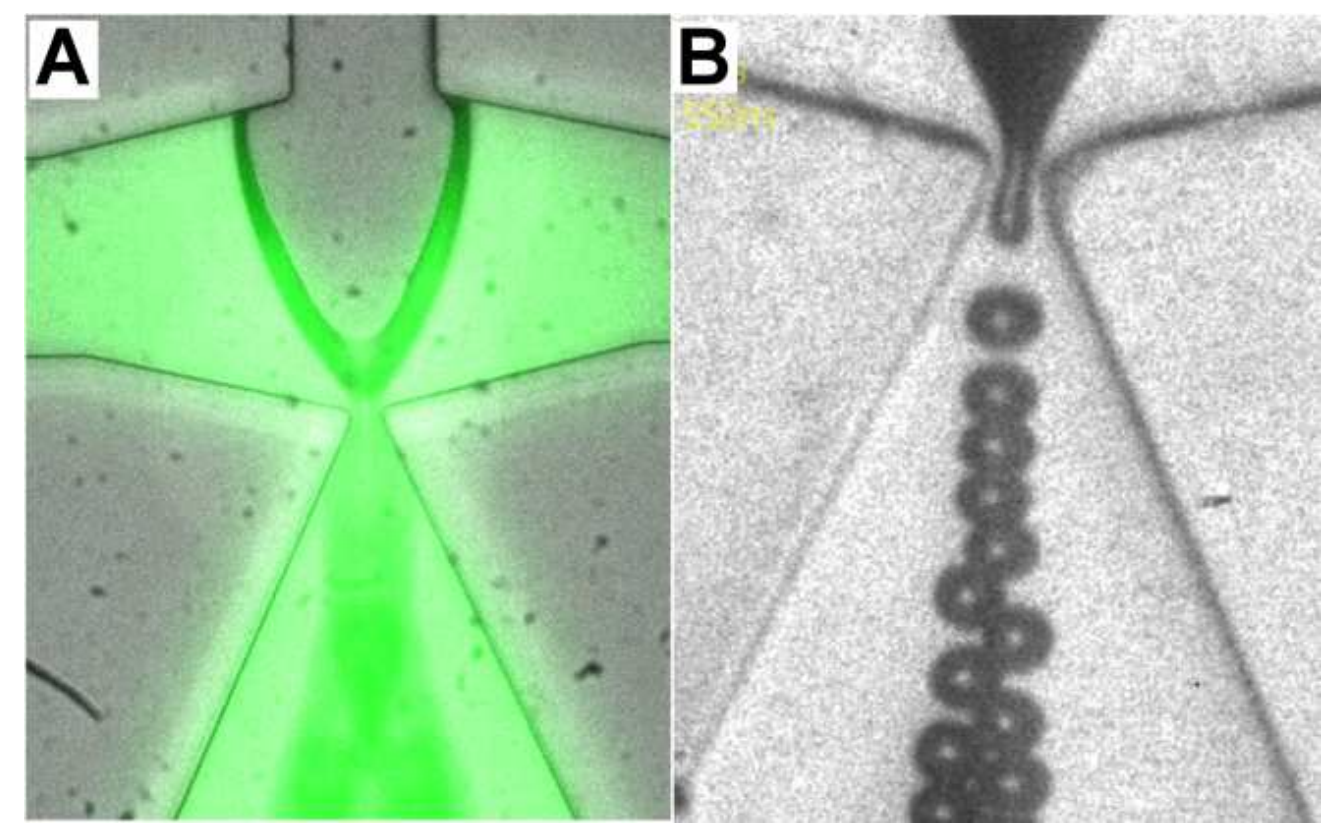

Figure 5.2: (A) Image of calcein incorporated into the liquid phase of the FFMD. (B) High speed image showing microbubble production (scale bar $=10 \mu \mathrm{m}$ ).

membranes. Percent delivery was calculated as the total number of cells that internalized calcein divided by the total number of cells on the membrane prior to insonation. Percent death was calculated as the sum of PI positive and dislodged cells divided by the total number of cells prior to insonation. Dislodged cells were calculated as the total number of cells pre-insonation minus the total number of cells post-insonation.

\subsection{Results}

\subsubsection{Microbubble characterization}

Microbubbles with diameters between $8-17 \mu \mathrm{m}$ were produced at rates between $0.1-0.5 \times 10^{6}$ microbubbles/s (Fig. 5.2B). The addition of calcein $(10 \mathrm{mg} / \mathrm{ml})$ directly to the liquid phase did not affect microbubble production (Fig. 5.2A). 


\subsubsection{Microbubble stability}

The FFMD was optically calibrated to produce $8 \mu \mathrm{m}$ diameter microbubbles. The microbubbles did not expand when introduced into the air-saturated medium used to acquire Coulter counter measurements. The number of microbubbles present versus time was fitted to an exponential decay model $\left(\mathrm{R}^{2}=0.97\right)$ and the half-life was calculated to be 18.2 seconds (Fig. 5.3). No microbubbles larger than $25 \mu \mathrm{m}$ were observed as measured by the Coulter counter and confirmed by brightfield optical microscopy. After 120 seconds, fewer than $1 \%$ of the initial population of microbubbles were detected above the $5 \mu \mathrm{m}$ lower threshold of the Coulter counter.

\subsubsection{Drug Delivery - pressure}

A flow rate of $9 \mathrm{~mL} / \mathrm{s}$ was used in this study. This flow rate resulted in a calculated wall shear stress consistent with cited values for arterial shear stress in vivo [159]. Flow alone did not cause cell dislodgement. In addition, no calcein delivery was observed when either ultrasound or microbubbles were applied alone. Negligible calcein delivery was observed for any of the control conditions.

Microbubble diameter and production rate were held constant at $10 \mu \mathrm{m}$ and 300,000 microbubbles/second, respectively, for all delivery conditions. Delivery was observed at peak negative pressures as low as $100 \mathrm{kPa}$ (Fig. 5.4A), and no statistically significant differences in delivery or death were observed between pressures of $100 \mathrm{kPa}$ and $300 \mathrm{kPa}$ (Fig. 5.5B). Maximum delivery was $82 \%$. In all cases cell death was less than $20 \%$. 


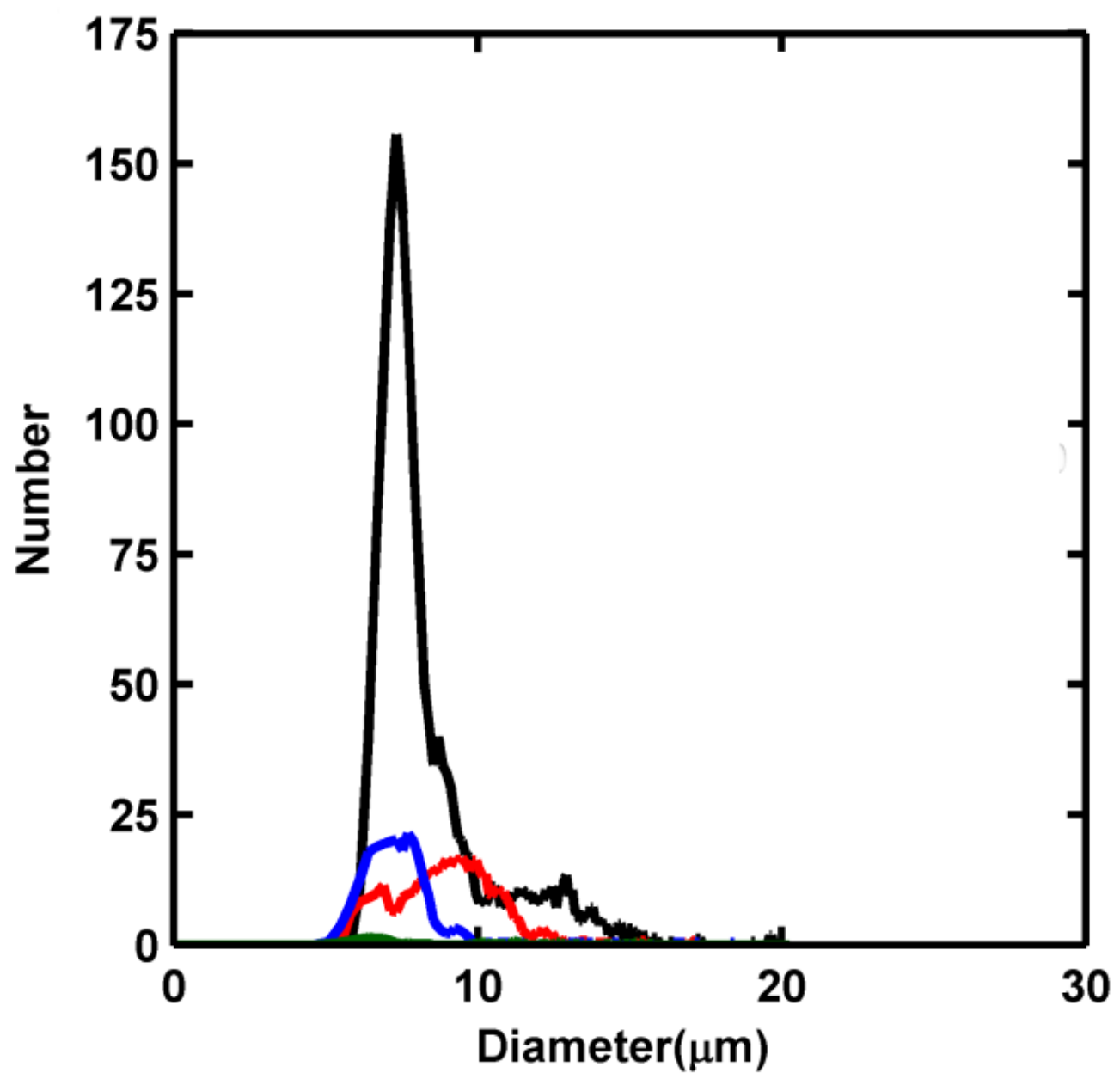

Figure 5.3: Size distribution of the FFMD-produced microbubbles as a function of time. Initial microbubble diameter were measured to be $8 \mu \mathrm{m}$ at the FFMD nozzle prior to placement of the FFMD into air-saturated saline for the diameter measurement. The half-life was calculated to be 18.2 seconds.

\subsection{Discussion}

\subsubsection{FFMD and microbubble characterization}

Microbubble stability was evaluated by a Coulter counter in a continuously stirred, airsaturated environment to more closely approximate the environment of blood in vivo [161]. The mean microbubble diameter, as measured by the Coulter counter, expanded slightly following production then quickly decreased as the gas diffused out of the microbubble shell (Fig. 5.3). The initial expansion may be due to the influx of oxygen from the air saturated 

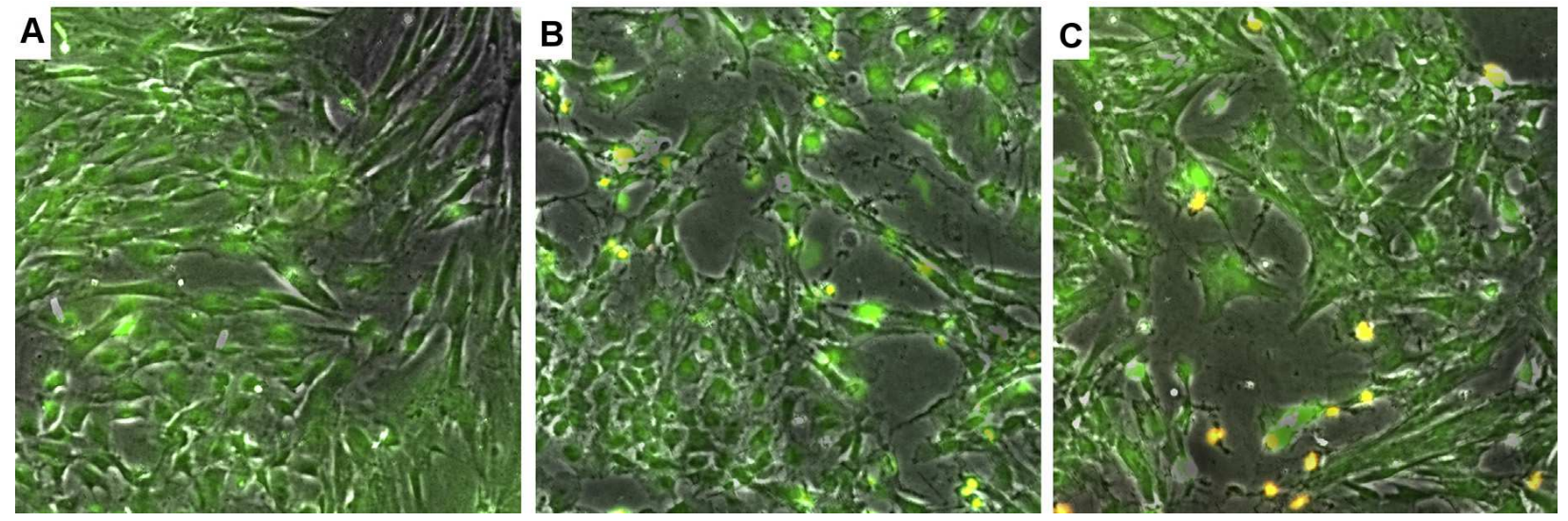

Figure 5.4: Fluorescence microscopy images of rat aortic smooth muscle cells following ultrasoundmediated calcein delivery. Green fluorescence corresponds to cells that have internalized calcein. Orange corresponds to cells that are dead (scale bar $=50 \mu \mathrm{m}$ ). (A-C) No difference in calcein delivery was observed at peak negative pressures of 100, 200, and $300 \mathrm{kPa}$, respectively. For all pressure conditions, flow rate was $9 \mathrm{ml} / \mathrm{s}$, and the microbubbles were $10 \mu \mathrm{m}$ in diameter and produced at a rate of 300,000 microbubbles/s.

media into the pure nitrogen microbubble. Microbubbles produced via sonication, composed of decafluorobutane, and stabilized with a lipid-shell showed no change in diameter or number when monitored for up to 15 minutes (data not shown). Furthermore, microbubbles with diameters that were multiples of the mean diameter were not observed, suggesting that there was minimal microbubble coalescence. This lack of microbubble diameter growth, either due to gas influx or coalescence, is consistent with in vivo reports on microbubble dissolution in the presence of large partial pressure gradients, suggesting that microbubble coalescence with ambient gases is of low risk [162].

In this work, the model drug was intentionally not placed in the FFMD liquid phase, since adjustment of the flow rate to achieve the desired microbubble diameter or production rate would have altered the drug dispensation concentration. To ensure consistent calcein concentration, calcein was co-administered with the microbubbles via a separate tube attached to the FFMD. The calcein concentration could then be held constant regardless of the liquid flow rate of the FFMD or the fluid flow rate in the flow chamber. However, the presence of calcein in the liquid phase did not disrupt microbubble formation within the FFMD (Fig. 5.2A), indicating that calcein, or another drug of choice that is either soluble in the 


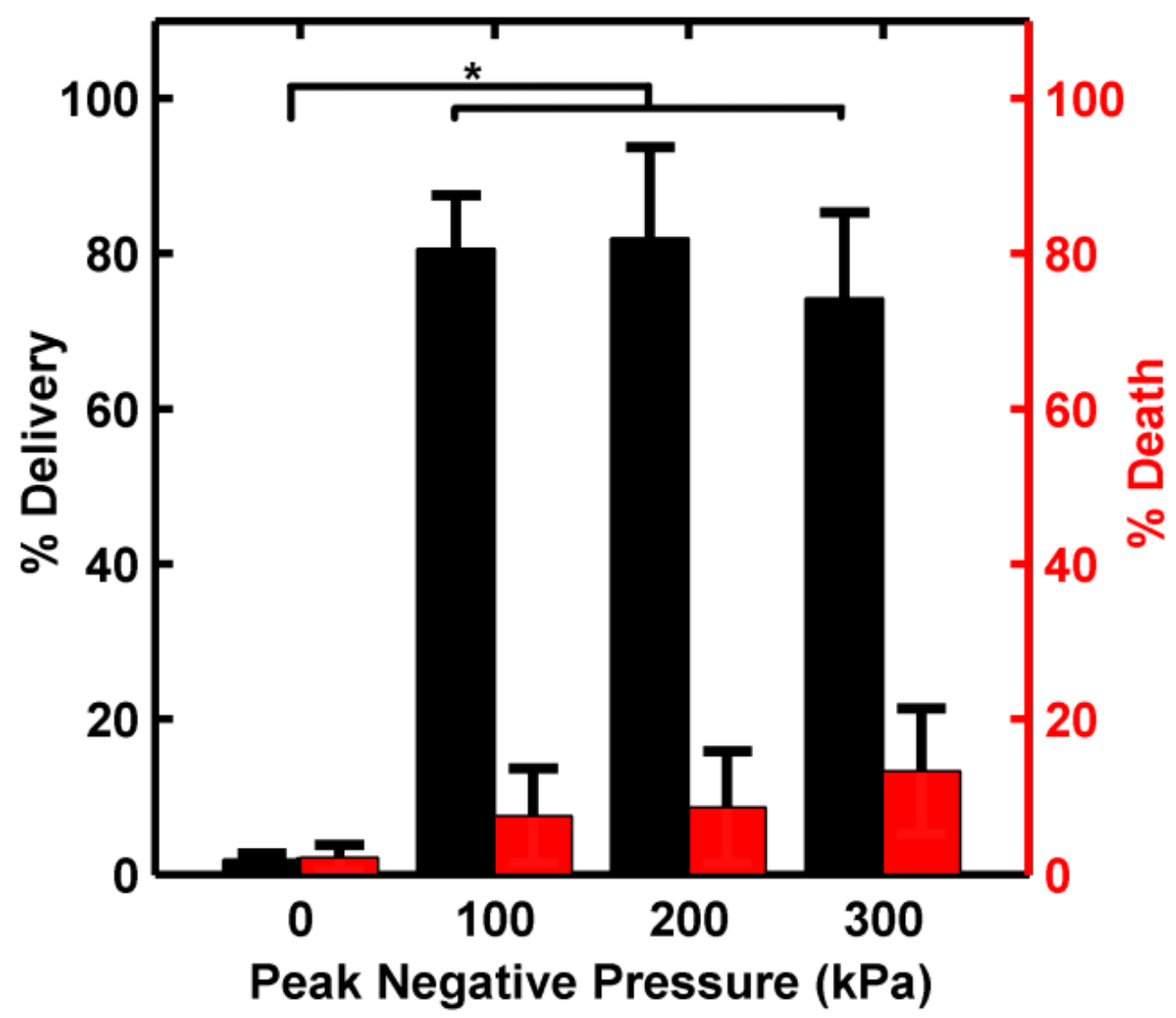

Figure 5.5: Delivery efficiency and percent cell death versus peak negative pressure. No significant difference in delivery or death was observed for pressures between 100 and $300 \mathrm{kPa}(*=\mathrm{p}<0.01$, $\mathrm{n}>3$ ). Flow rate was $9 \mathrm{ml} / \mathrm{s}$. Microbubbles were $10 \mu \mathrm{m}$ in diameter and produced at a rate of $300,000 \mathrm{MB} / \mathrm{s}$.

microbubble shell or liquid solvent phase, could be incorporated directly within the FFMD if need be.

\subsubsection{Drug delivery and pressure}

In the presence of ultrasound, the microbubbles enhanced cellular uptake of calcein (Fig. 5.5). Significantly, the combination of in situ produced microbubbles and ultrasound was able to achieve $80 \%$ delivery at pressures as low as $100 \mathrm{kPa}$. This level of enhanced delivery is significantly higher than what has been observed in other in vitro studies [160], most of 
which were performed under static conditions and using microbubbles with diameters less than $4 \mu \mathrm{m}$. The inclusion of flow to the in vitro model may have prevented the microbubbles from stagnating near the cells, thus reducing excessive pore formation and subsequent cell death on any given cell. Simultaneously, oscillation dynamics of large diameter microbubbles compared to small diameter microbubbles may enable optimal pore formation that enables drug uptake without preventing membrane resealing.

I envision this technology as a complement to existing catheter-based interventions. The microbubbles could be used to delineate the borders of a plaque or aneurysm using IVUS imaging [167], guide stent deployment, or deliver a post-procedure therapy that cannot be administered systemically [147, 145]. Furthermore, since microbubbles can be produced and administered on-demand, in situ microbubble production could enable enhanced imaging contrast and therapy over the entire duration of a procedure rather than being limited to one or two bolus injections [78]. Thus, this technology may provide significant benefits in terms of real-time guidance and improved drug delivery to vascular targets without any disadvantages beyond those already associated with current catheter-based interventions.

\subsection{Conclusions}

In this chapter, I report on enhanced cell delivery through the combination of ultrasound and in situ produced microbubbles. Microbubbles were produced in real-time and in close proximity to the therapeutic target. Importantly, in situ production removed the stability requirement, thus enabling investigation of unconventional, unstable, large diameter microbubbles. Drug delivery up to $80 \%$ and cell death less than $20 \%$ were achieved at insonation pressures as low as $100 \mathrm{kPa}$. These excellent delivery rates may be due to improved simulation of an in vivo environment and utilization of large diameter microbubbles. 


\section{Chapter 6}

\section{In vivo imaging of}

\section{microfluidic-produced microbubbles ${ }^{1}$}

\subsection{Abstract}

In culmination of the previous chapters which develop the paradigm of in situ microfluidic production through in vitro models, this chapter is the first report of microfluidic-produced microbubbles directly administered into a mouse tail vein for in vivo image contrast. Microbubbles composed of nitrogen gas and stabilized with 3\% bovine serum albumin and $10 \%$ dextrose were injected for 10 seconds into wild type C57BL/6 mice, via a tail-vein catheter. Short-axis images of the right and left ventricle were acquired at $12.5 \mathrm{MHz}$ and image intensity over time was analyzed. Microbubbles were produced on the order of $10^{5}$ microbubbles/s and were observed in both the right and left ventricles. The median rise time, duration, and decay time within the right ventricle were $2.9,21.3$, and $14.3 \mathrm{~s}$, respectively. All mice survived the procedure with no observable respiratory or heart rate distress despite microbubble diameters as large as $19 \mu \mathrm{m}$.

\footnotetext{
${ }^{1}$ This chapter will be submitted for peer-review:

A. H. Dhanaliwala, A. J. Dixon, D. Lin, J. L. Chen, A. L. Klibanov, and J. A. Hossack, "In vivo imaging of microfluidic-produced microbubbles," Biomedical Microdevices, vol. Submitted, 2014.
} 


\subsection{Background}

As a step towards in situ microbubble production, I demonstrated that microfluidics can produce a sufficient quantity of microbubbles to produce acoustic contrast (chapter 2), and effect drug delivery (chapter 5) in in vitro settings. In addition, albumin was specifically investigated as a shell material for the production of an intentionally unstable microbubble that could minimize the emboli risk of large diameter microbubbles (chapter 4). Again, these transiently-stable microbubbles were shown capable of producing a strong acoustic signal when imaged with a clinical ultrasound scanner. In this chapter I present the first report on the administration of microbubbles directly from the output of a microfluidic device, through a tail-vein catheter, and into the mouse vasculature for in vivo ultrasound image enhancement. The two major goals of this study were to investigate (1) whether transiently-stable microbubbles produced by a flow-focusing microfluidic device and directly administered into the vasculature could provide sufficient acoustic contrast in vivo; and (2) whether transient-stability would abrogate any acute health effects of large diameter microbubbles.

\subsection{Materials and Methods}

\subsubsection{Microfluidic production of microbubbles}

Microbubbles were fabricated using a flow-focusing microfluidic device (FFMD) (Fig. 6.1) as described in section 4.3. The final dimensions of the device were: $35 \mu \mathrm{m}$ gas channel width, $50 \mu \mathrm{m}$ liquid channel width, $22 \mu \mathrm{m}$ channel height, and $7 \mu \mathrm{m}$ nozzle orifice width. The liquid phase consisted of $3 \%$ bovine serum albumin (BSA) and 10\% dextrose (Sigma-Aldrich, St. Louis, MO) in isotonic saline. The gas phase consisted of $99.998 \%$ pure nitrogen (GTS Welco, Richmond, VA). Microbubbles were produced as described in section 2.3.2. 

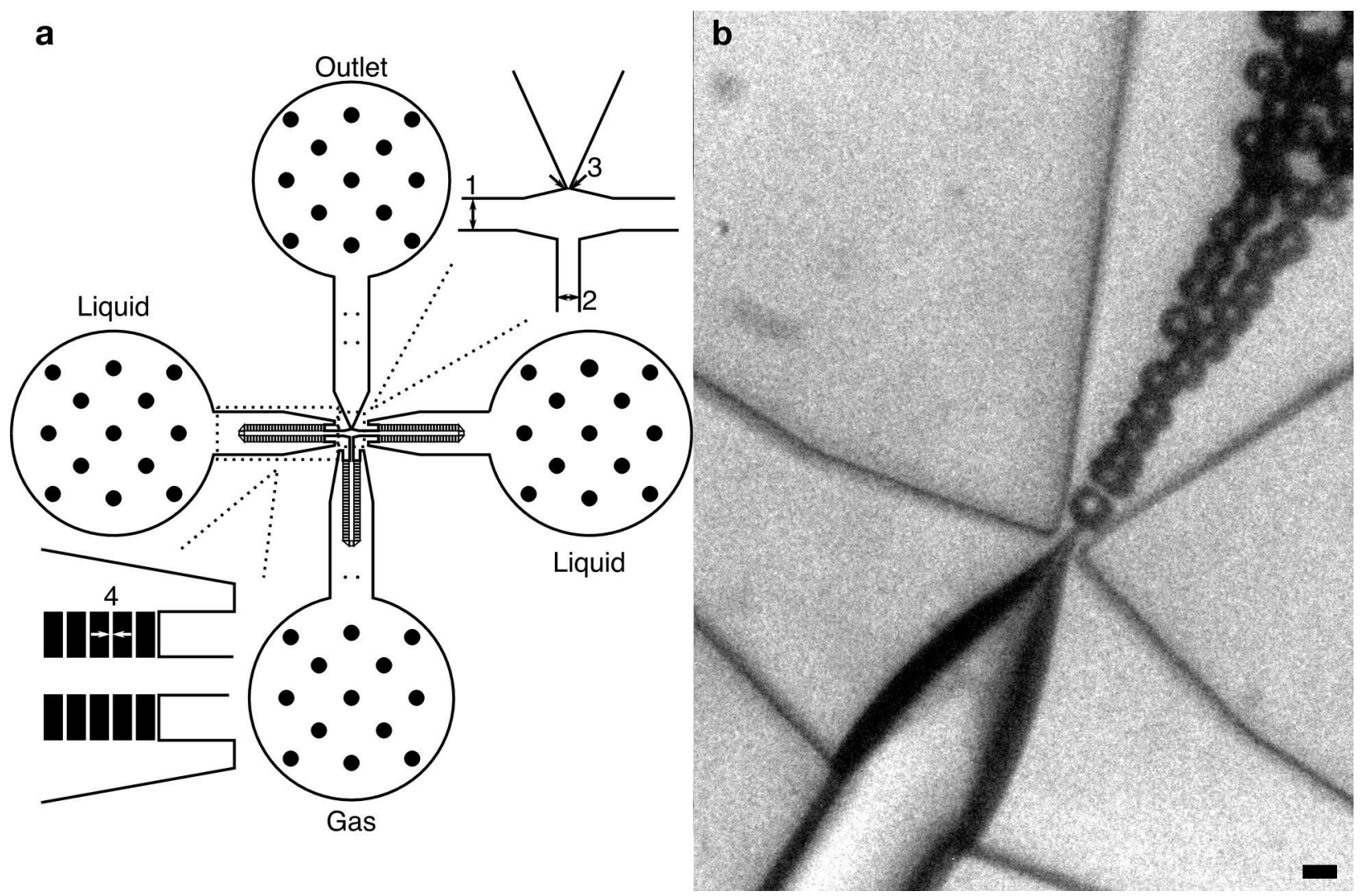

Figure 6.1: a) Schematic of the flow-focusing microfluidic device. Maximum dimensions of the device were $6.6 \mathrm{~mm} \times 7 \mathrm{~mm}$. Black circles represent support posts. Width of the liquid channel (1), gas channel (2), and nozzle orifice (3) were 50, 35, and $7 \mu \mathrm{m}$, respectively. Filter channel widths (4) were $5 \mu \mathrm{m}$. b) Single frame from a high speed video showing microbubble production. The gas cone in the center is sheared at the nozzle orifice by the liquid phase to produce microbubbles. Scale bar is $10 \mu \mathrm{m}$.

A separate population of albumin-shelled fluorocarbon microbubbles were fabricated via sonication [168] and served as a control. A 1\% solution of serum albumin in isotonic saline was purged with octafluoropropane gas $\left(\mathrm{C}_{3} \mathrm{~F}_{8}\right.$, Scott Medical Products, Plumsteadville, PA). Octafluoropropane was then layered above the solution in a flask and the solution was sonicated for $30 \mathrm{~s}$ with an ultrasonic tip sonicator (XL2020, Misonix, Farmingdale, NY) equipped with an extended 1/2" titanium probe. Prepared microbubbles were stored in a sealed vial with a octafluoropropane headspace at $4^{\circ} \mathrm{C}$. 


\subsubsection{In vivo imaging}

All animal imaging was in compliance with a University of Virginia Animal Care and Use Committee approved protocol. C57BL/6 mice were placed on an animal handling platform (VisualSonics, Toronto, ON) that enabled warming and monitoring of heart rate, respiration rate, and temperature. The mice were anesthetized with $2 \%$ isofluorane and $98 \%$ room air for the duration of the study. One end of an intravenous catheter was inserted into the tail vein of a mouse, while the other end was inserted directly into the outlet of the FFMD. A high frequency, small animal ultrasound scanner (Vevo 2100, VisualSonics, Toronto, ON) was used to image the microbubbles in the right and left ventricles via a short-axis view of the heart. The microbubbles were imaged at $12.5 \mathrm{MHz}$ and $10 \%$ transmit power, and both B-mode (1 pulse, 1 cycle, 15 dB gain) and nonlinear contrast mode (2 pulse, 4 cycle, amplitude modulation, $27 \mathrm{~dB}$ gain) images were acquired. The FFMD was affixed to a microscope to enable continuous monitoring of microbubble production rate and diameter as described in section 2.3.2.

A total of six mice were each imaged three times yielding a total of 18 imaging sessions. For each imaging session, the outlet of the FFMD was directly connected to the tail vein catheter for $10 \mathrm{~s}$, and a short-axis cine of the heart was acquired from just prior to microbubble injection until the image contrast in the ventricles returned to baseline. The first two imaging sessions were separated by an hour. The final imaging session was performed one week later.

For comparison, five imaging sessions were acquired using the agitation-produced microbubbles. Prior to use, diameter and concentration of the agitation-produced microbubbles were measured with a Coulter Counter (Multisizer III, Beckman Coulter, Brea, CA). The microbubbles were then diluted in isotonic saline such that an equivalent number of microbubbles were injected over $10 \mathrm{~s}$ as were used in the microfluidic-microbubble imaging studies.

Beamformed, envelope-detected, 8-bit, B-mode images were exported from the ultrasound scanner and analyzed in MATLAB (Mathworks, Natick, MA). Regions of interest (ROIs) 
were placed in the right and left ventricle to calculate mean image intensity over the duration of the B-mode cine. Intensity curves were background subtracted using the mean of the first $0.33 \mathrm{~s}$ of the image prior to contrast injection, and normalized to the peak intensity in the right ventricle. Rise times were calculated as the time from $25 \%$ to $75 \%$ image intensity. Decay times were calculated as the time from $75 \%$ to $25 \%$ image intensity after peak intensity. Duration was calculated as the time between $50 \%$ image intensity before and after peak intensity. Microbubble diameter and production rate were determined from high speed camera stills using ImageJ (NIH, Bethesda. MD).

\subsubsection{Microbubble oscillation model}

To investigate the effects of imaging frequency and microbubble size on the received acoustic signal, the Keller Miksis-polytropic model of microbubble oscillation was analyzed ${ }^{2}$ [169]:

$$
\begin{aligned}
\left(1-\frac{\dot{R}}{c}\right) R \ddot{R}+\frac{3}{2} \dot{R}^{2}\left(1-\frac{\dot{R}}{3 c}\right)= & \\
& \left(1+\frac{\dot{R}}{c}\right) \frac{1}{\rho_{L}}\left[p_{\text {int }}-p_{\text {drive }}\left(t+\frac{R}{c}\right)-p_{a m b}\right]+\frac{R}{\rho_{L} c} \frac{d p_{\text {int }}}{d t},
\end{aligned}
$$

where $R$ is the microbubble radius, $\dot{R}$ is the microbubble wall velocity, $\ddot{R}$ is the microbubble wall acceleration, $\rho_{L}$ is the density of surrounding liquid, $c$ is the speed of sound in surrounding liquid, $p_{\text {drive }}$ is the time delayed external driving acoustic pulse, $p_{a m b}$ is the ambient liquid pressure, and $p_{i n t}$ is the pressure inside the gas microbubble:

$$
p_{i n t}=\left(p_{a m b}+\frac{2 \sigma}{R_{0}}\right)\left(\frac{R_{0}}{R}\right)^{3 \kappa}-\frac{2 \sigma}{R}-\frac{4 \eta_{L} \dot{R}}{R}
$$

where $\sigma$ is the gas-liquid surface tension, $R_{0}$ is the initial microbubble radius, $\eta_{L}$ is the viscosity of the surrounding liquid, and $\kappa$ is the polytropic exponent. Radius-time curves

\footnotetext{
${ }^{2}$ In comparison to the original Rayleigh-Plesset model, the Keller Miksis model includes a correction term to account for the compressibility of the liquid medium at high pressures.
} 
Table 6.1: Microbubble oscillation simulation parameters

\begin{tabular}{ccc}
\hline Symbol & Parameter & Value \\
\hline \hline & Peak negative pressure & $100 \mathrm{kPa}$ \\
$\eta_{L}$ & Liquid viscosity & $0.002 \mathrm{~Pa} \mathrm{~s}^{-3}$ \\
$\rho_{L}$ & Liquid density & $1000 \mathrm{~kg} \mathrm{~m}^{-3}$ \\
$c$ & Speed of sound & $1500 \mathrm{~m} \mathrm{~s}^{-1}$ \\
$p_{a m b}$ & Ambient pressure & $101.3 \mathrm{kPa}$ \\
$\sigma$ & Surface tension & $0.071 \mathrm{~N} \mathrm{~m}^{-1}$ \\
$\kappa$ & Gas polytropic exponent & 1.4 \\
$r$ & Distance from microbubble & $1 \mathrm{~cm}$ \\
\hline Liquid properties are those of water. Gas properties are those of \\
nitrogen. Viscosity was doubled to account for thermal damping.
\end{tabular}

were solved using the $O D E 45$ numerical solver and a tolerance of $1 \times 10^{-5}$ in MATLAB. Gaussian-ramped, $100 \mathrm{kPa}, 4$ cycle insonation pulses were used to insonate the microbubbles with initial radii between 0.5 and $10 \mu \mathrm{m}$. The properties of nitrogen were used for the internal gas and the properties of water were used for the liquid. The viscosity of water was doubled to account for thermal damping [170]. Additional simulation parameters are listed in Table 6.1.

Finally, a relationship between pressure and velocity potential [171]:

$$
P_{M B}=\frac{\rho_{L}}{r}\left(R^{2} \ddot{R}+2 R \dot{R}^{2}\right)
$$

was used to convert radius-time curves to a predicted pressure wave, where $P_{M B}$ is the pressure amplitude at a distance $r$ from the microbubble.

\subsubsection{Microbubble dissolution model}

Given the difficulty in directly measuring microbubble lifetime in vivo, a model of microbubble dissolution was analyzed to obtain an estimated microbubble lifetime. The original EpsteinPlesset model of gas dissolution [172] was modified in order to incorporate the effect of blood pressure on microbubble lifetime [103]:

$$
\dot{R}=-D_{N} L_{N} \frac{p_{\text {blood }}+\frac{2 \sigma}{R}}{p_{a m b}+\frac{4 \sigma}{3 R}}\left[\frac{1}{R}\right],
$$


Table 6.2: Microbubble dissolution simulation parameters

\begin{tabular}{ccc}
\hline Symbol & Parameter & Value \\
\hline \hline$p_{\text {blood }}$ & Overpressure in blood & $4.3 \mathrm{kPa}[103]$ \\
$D_{N}$ & $\mathrm{~N}_{2}$ gas diffusion coefficient & $2.01\left(25^{\circ} \mathrm{C}\right), 2.83\left(40^{\circ} \mathrm{C}\right) \times 10^{-9} \mathrm{~m}^{2} \mathrm{~s}^{-1}[175]$ \\
$L_{N}$ & $\mathrm{~N}_{2}$ gas Ostwald coefficient & $15880\left(25^{\circ} \mathrm{C}\right), 14000\left(40^{\circ} \mathrm{C}\right) \times 10^{-6}[176]$ \\
\hline
\end{tabular}

where $D$ is the diffusion coefficient of nitrogen gas in water, $L$ is the Ostwald coefficient of nitrogen gas in water, and $p_{\text {blood }}$ is the overpressure due to systemic blood pressure. In the model, the medium is considered fully gas saturated, the gas properties of nitrogen in blood are considered similar to the gas properties in water, and oxygen influx and non-steady state effects are neglected. Since the microbubbles have a non-crosslinked protein shell, the effect of the shell on gas diffusion $[173,174]$ is also assumed negligible. MATLAB was used to numerically solve the equation using the ODE45 solver with a tolerance of $1 \times 10^{-8}$. Complete dissolution was set as the time when $R=0$. Simulation parameters are listed in Table 6.2.

\subsection{Results}

\subsubsection{In vivo imaging}

Microbubbles with diameters between 9.1 and $19.8 \mu \mathrm{m}$ and with production rates between 250,000 and 830,000 microbubble/s (Fig. 6.2) were administered to the mice.

Following injection of the microfluidic-produced microbubbles, the right ventricle, but not the left ventricle, was clearly visualized (Fig. 6.3). In only $63 \%$ of the trials was the image intensity in the left ventricle greater than 10 a.u. on the 8-bit scale, and on average the peak image intensity in the left ventricle was only $18 \%$ of the peak image intensity of the right ventricle (Fig. 6.5). For comparison, $20 \mu \mathrm{L}$ of $1.8 \mu \mathrm{m}$ diameter agitation-produced microbubbles at a concentration of $250 \times 10^{6}$ microbubbles $/ \mathrm{mL}$ were injected over $10 \mathrm{~s}$ into a mouse via a tail-vein catheter and a short-axis view of the heart was acquired. When the 

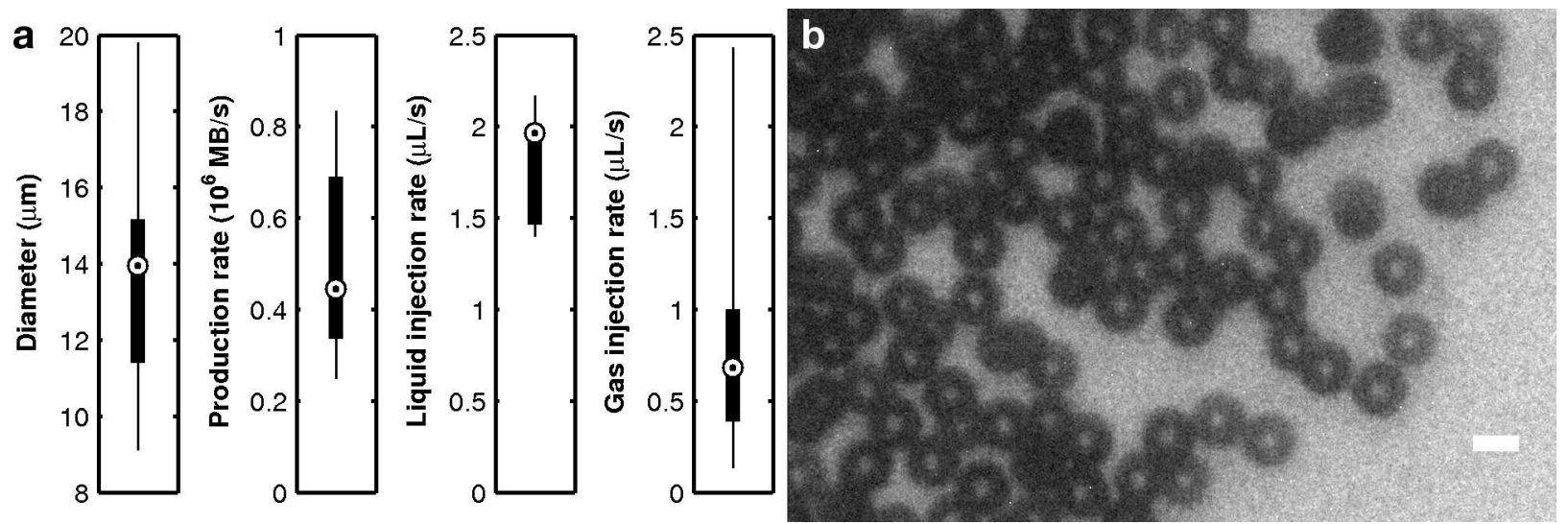

Figure 6.2: (a) Microfluidic-produced microbubble characteristics across all imaging procedures. Total injected gas is the product of microbubble volume and production rate. Filled box represents $25^{\text {th }}-75^{\text {th }}$ percentile and circle with $\operatorname{dot}(\odot)$ is the median. (b) Image of microbubbles within the FFMD outlet channel just prior to entering the tail vein catheter. Scale bar $=10 \mu \mathrm{m}$.

agitation-produced microbubbles were injected, contrast was clearly observed in both the right and left ventricle and the median duration of contrast was $70 \%$ longer (Fig. 6.4). The median contrast level in the right ventricle with the agitation-produced microbubbles was only $70 \%$ of the signal observed with the microfluidic-produced microbubbles; however, the median contrast level in the left ventricle was four-fold higher.

Across all trials, the median rise time, duration, and decay time for the microfluidicproduced microbubbles were 2.9, 21.3, and $14.3 \mathrm{~s}$, respectively. Across all trials, the median rise time, duration, and decay time for the agitation-produced microbubbles were 2.4, 16.5, $35.8 \mathrm{~s}$, respectively. There was no statistical significant difference between these values as calculated by the Wilcoxon rank sum test ${ }^{3}$. Median peak intensity for microfluidic-produced and agitation-produced microbubbles in the right ventricle were 80 and 56 a.u., respectively, and the difference was observed to be statistically significant per the Wilcoxon rank sum test $(\mathrm{p}=0.01)($ Fig. 6.5). No correlation was observed between peak intensity and microbubble diameter, production rate, or volume-production rate (Fig. 6.6). Similarly, no correlation was observed between rise time, duration, and decay time, and microbubble diameter or production rate, or volume-production rate.

\footnotetext{
${ }^{3}$ Given the small sample size and the uncertainty of the underlying distribution, the data cannot be assumed to be normal and a t-test is unlikely to be valid.
} 


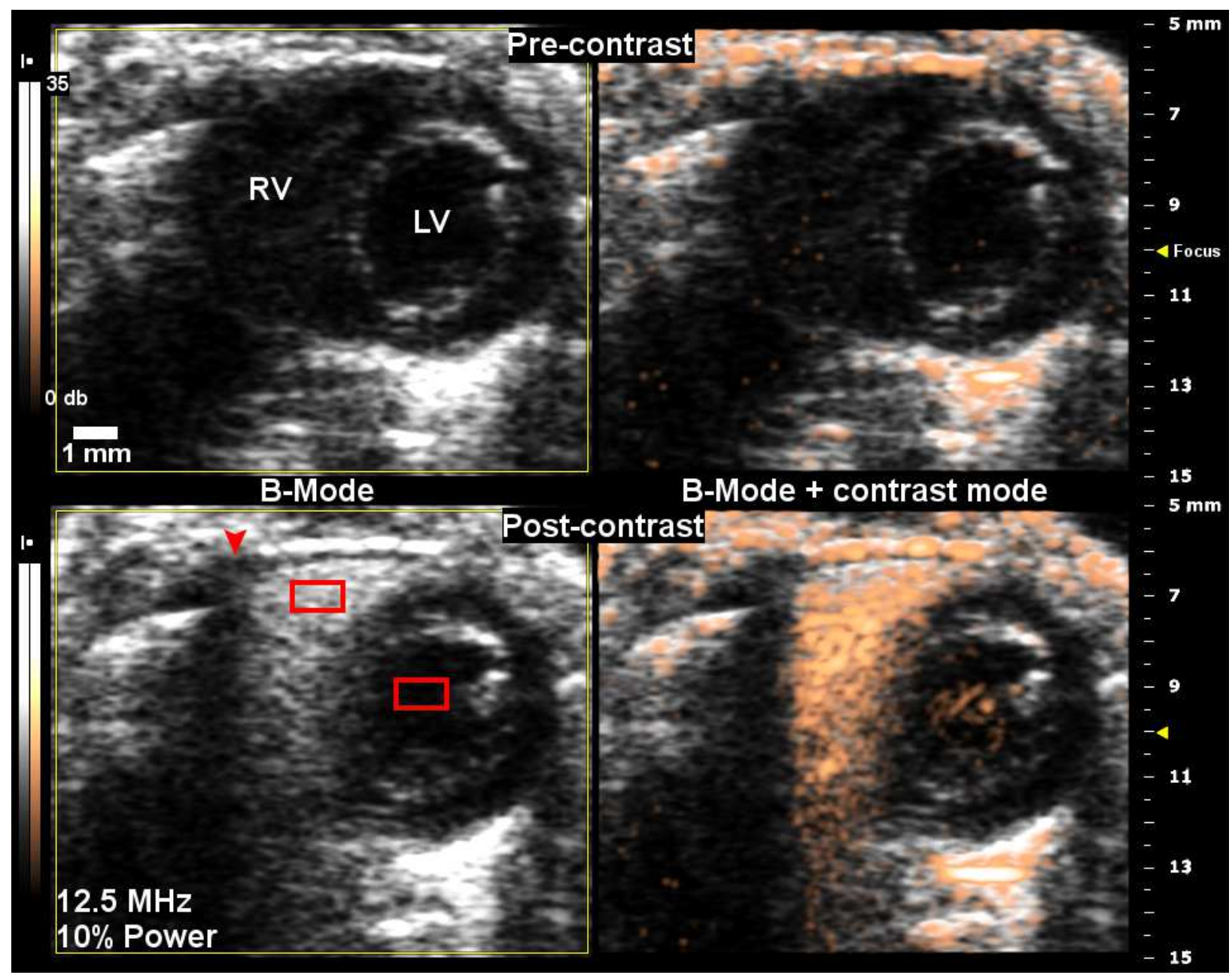

Figure 6.3: (Top) Pre-contrast (Bottom) post-contrast images of the right (RV) and left (LV) ventricles following injection of microfluidic-produced microbubbles. B-Mode (Left) and B-Mode with nonlinear contrast overlay (Right). The injected microbubbles had a diameter of $14.4 \pm 1 \mu \mathrm{m}$ and were produced at a rate of 435,000 microbubble/s. Peak B-Mode image intensity in the right ventricle and left ventricle were 108 and 10 a.u., respectively. Red rectangles indicate region of interest (ROI) within which image intensity was extracted. Arrowhead indicates image dropout due to sternal artifact. Ultrasound parameters: $12.5 \mathrm{MHz}$ center frequency, $10 \%$ transmit power, $15 \mathrm{~dB}$ B-Mode gain, $27 \mathrm{~dB}$ contrast-mode gain, $35 \mathrm{~dB}$ dynamic range.

All animals survived all imaging procedures. During imaging, median heart rate across all trials was 460 beats per minute and median respiration rate across all trials was 160 breaths per minute. We have observed that for our mice under isofluorane anesthesia, the normal bounds for the heart rate and the respiratory rate are 400-500 beats per minute and 90-180 breaths per minute, respectively, at $37^{\circ} \mathrm{C}$. One week post-imaging, all animals were observed to be in good health. 
a
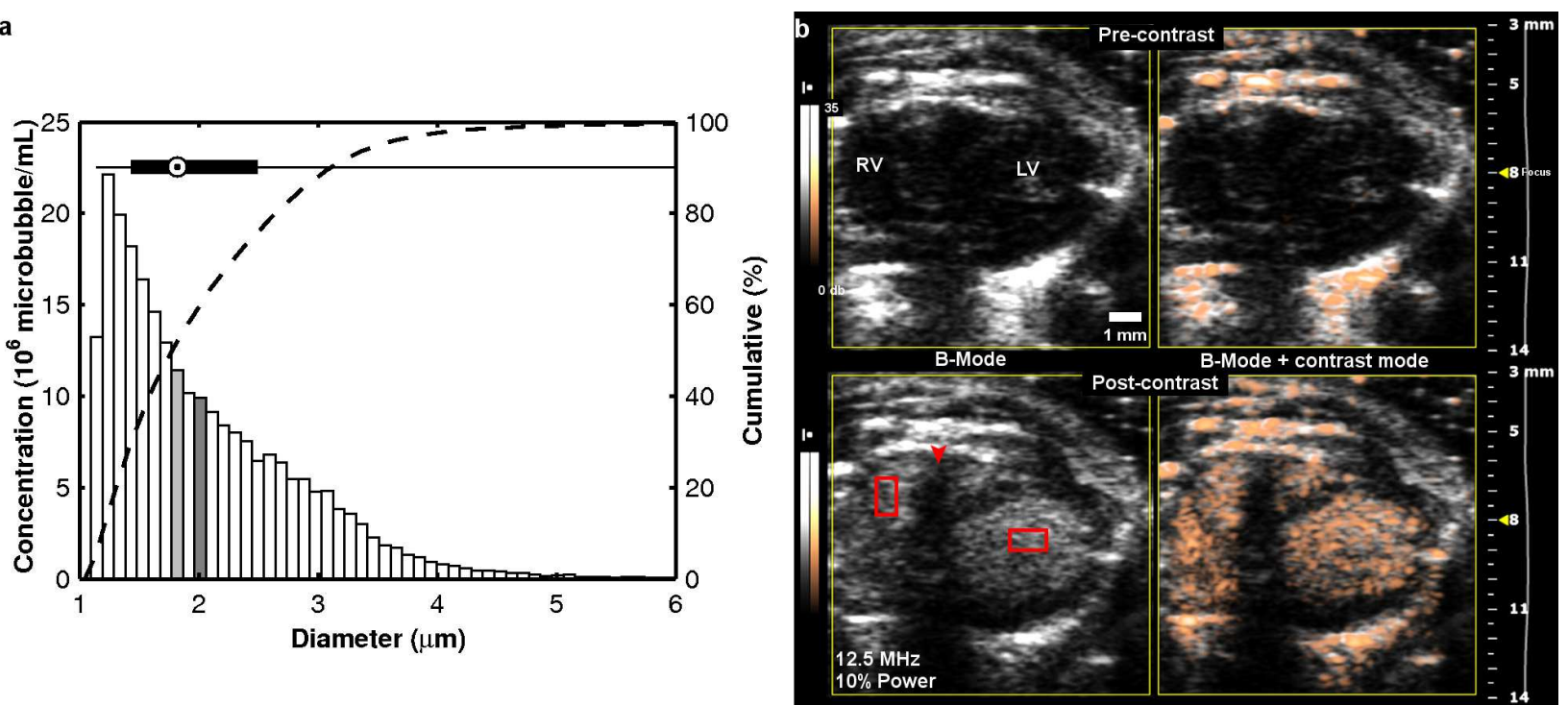

Figure 6.4: (a) Size distribution of agitation-produced microbubbles. Light and dark gray bars represent the median $(1.8 \mu \mathrm{m})$ and mean $(2.1 \mu \mathrm{m})$ diameter, respectively. Filled black box represents $25^{\text {th }}-75^{\text {th }}$ percentile. Dash line represents cumulative percentage $(90 \%<3.1 \mu \mathrm{m})$. b) Pre-contrast (top) post-contrast (bottom) images of the right (RV) and left (LV) ventricles following injection of agitation-produced microbubbles. B-Mode (Left) and B-Mode with nonlinear contrast overlay (Right). Peak B-Mode image intensity in the right ventricle and left ventricle were 65, and 63 a.u., respectively. Red rectangles indicate region of interest (ROI) within which image intensity was extracted. Arrowhead indicates image dropout due to sternal artifact. Ultrasound parameters: $12.5 \mathrm{MHz}$ center frequency, 10\% transmit power, $15 \mathrm{~dB}$ B-Mode gain, $27 \mathrm{~dB}$ contrast-mode gain, $35 \mathrm{~dB}$ dynamic range.

\subsubsection{Microbubble simulations}

\section{Received pressure}

Peak to peak pressure was calculated from the simulated echoes as the difference between the maximum and minimum pressure and normalized to the maximum value amongst all frequencies and diameters. Large $(>10 \mu \mathrm{m})$ microbubbles exhibited an increased maximal peak to peak pressure as compared to smaller microbubbles, with the trend linearly increasing after the resonance peak. In addition, the normalized pressure for diameters greater than $5 \mu \mathrm{m}$ were within $5 \%$ of each other (Fig. 6.7a). A sharp resonance peak is observed for $2.2 \mu \mathrm{m}$ diameter microbubbles at a frequency of $4 \mathrm{MHz}$ resulting in an equivalent peak to peak pressure of a $13 \mu \mathrm{m}$ diameter microbubble.

The energy of the simulated echo was calculated as the integral of the square of the 


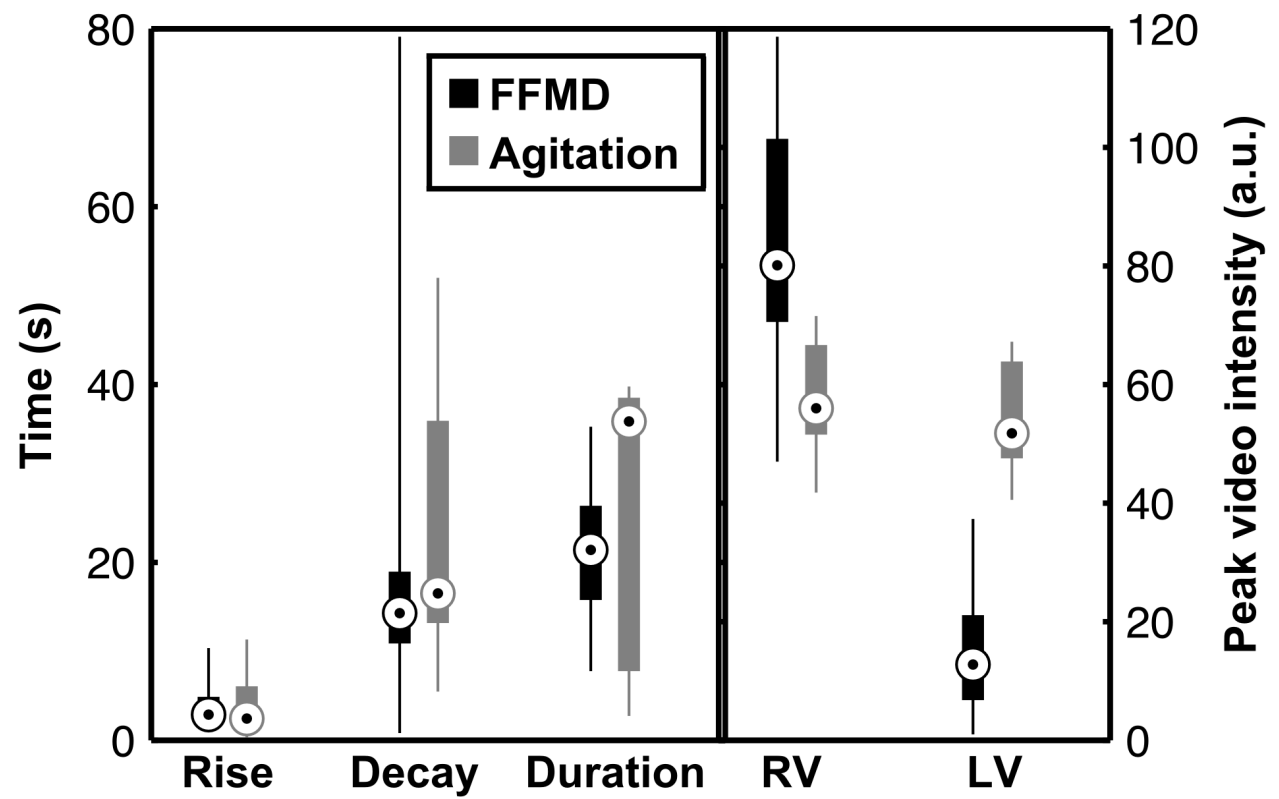

Figure 6.5: (Left) Rise time, decay time, and duration of image contrast in the right ventricle across all imaging trials. (Right) Peak video intensity in the right (RV) and left (LV) ventricle. Agitationproduced microbubbles exhibited a longer duration and a higher LV intensity. Microfluidic-produced microbubbles were observed to have the overall highest peak intensity. Filled box represents $25^{\text {th }}-$ $75^{\text {th }}$ percentile and circle with $\operatorname{dot}(\odot)$ is the median. Per the Wilcoxon rank sum test the difference in rise, decay, or duration time between microfluidic-produced and agitation-produced microbubbles was not statistically significant. The difference in image intensity within each ventricle between the microfluidic-produced and agitation-produced microbubbles was observed to be statistically significant $(\mathrm{RV}: \mathrm{p}=0.01 ; \mathrm{LV}: \mathrm{p}=0.0008)$.
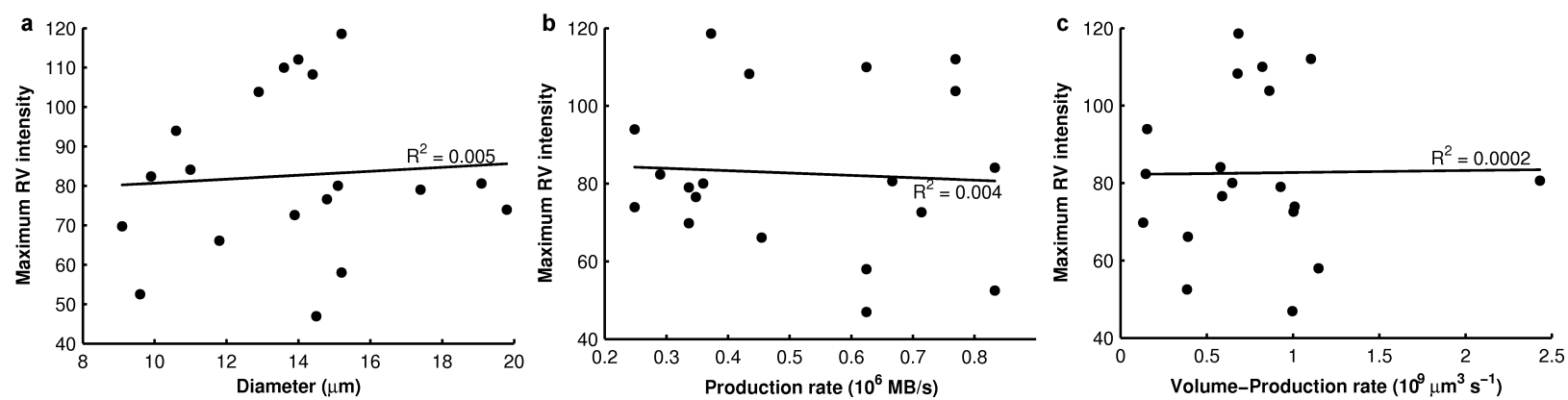

Figure 6.6: No significant correlation was observed between maximum image intensity in the right ventricle (RV) and either microbubble diameter, production rate or the product of microbubble volume and production rate (i.e. total gas). Lines represent a least squares linear regression with their respective $\mathrm{R}^{2}$ values.

absolute value of the signal. Each echo was normalized to the energy of their respective insonation pulse. All energy values were then normalized to the maximum energy amongst all 

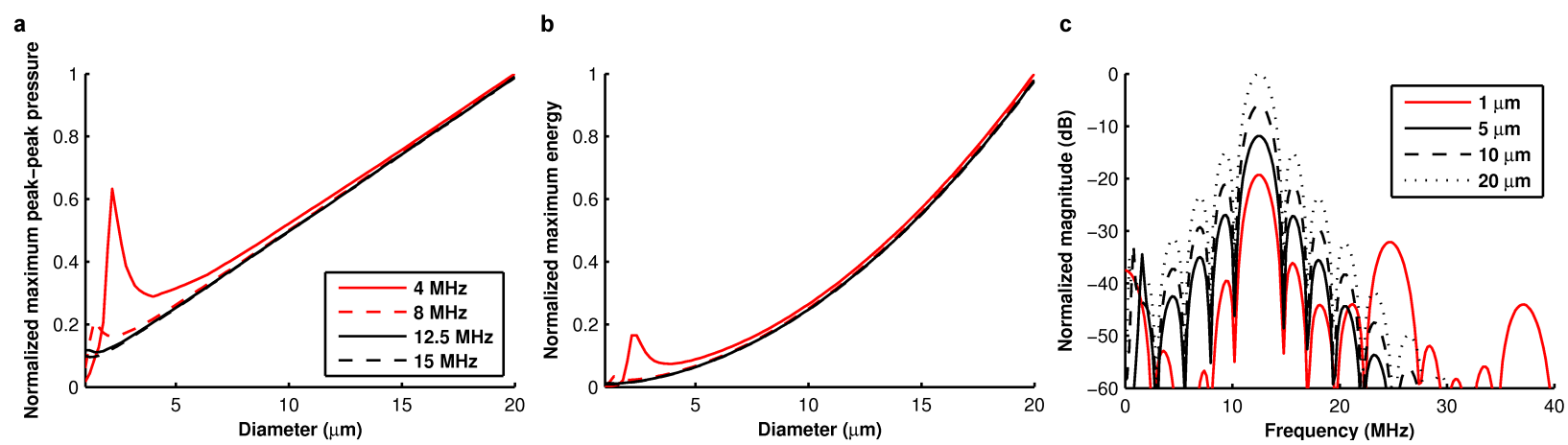

Figure 6.7: (a) Except when insonated at resonance microbubbles with increasing diameters produced echoes with increasing pressures. (b) The signal improvement due to resonance is abrogated when comparing energy versus pressure. (c) Normalized frequency spectra of microbubbles insonated with a $12.5 \mathrm{MHz}$ pulse. The magnitude of the fundamental frequency decreased with decreasing microbubble diameter. For a $1 \mu \mathrm{m}$ diameter microbubble harmonics were generated.

diameters and frequencies. When energies are compared, the $2.2 \mu \mathrm{m}$ diameter microbubble insonated at $4 \mathrm{MHz}$ now only has the equivalent energy of an $8.2 \mu \mathrm{m}$ diameter microbubble (Fig. 6.7b). Consistent with the linear increase in pressure observed in peak to peak pressure, the energy of the received echo increased quadratically following the resonance peak.

Finally, the frequency spectrum of the simulated echoes were compared for a $12.5 \mathrm{MHz}$ insonation pulse. As the microbubble diameter decreased, the magnitude of the fundamental frequency decreased, with a $20 \mathrm{~dB}$ decrease between 20 and $1 \mu \mathrm{m}$ diameter microbubbles (Fig. 6.7c). The spectrum of the $20 \mu \mathrm{m}$ diameter microbubble was observed to be identical in shape to the spectrum of the insonation pulse, while the spectrum of the $1 \mu \mathrm{m}$ diameter microbubble generated harmonics not present in the insonation pulse.

\section{Dissolution}

Dissolution of microbubbles with a range of diameters was solved over a range of surface tensions (Fig. 6.8). Isolines represent microbubble lifetime in seconds. Microbubble lifetime increased with increasing diameter and decreasing surface tension. Including the effects of a blood environment resulted in a decrease in microbubble lifetime (Fig. 6.8b) as compared to a microbubble at 1 atm $(101.3 \mathrm{kPa})$ and $25^{\circ} \mathrm{C}$ (Fig. 6.8a). Injection into the bloodstream results in an additional overpressure due to systemic blood pressure as well as changes in 

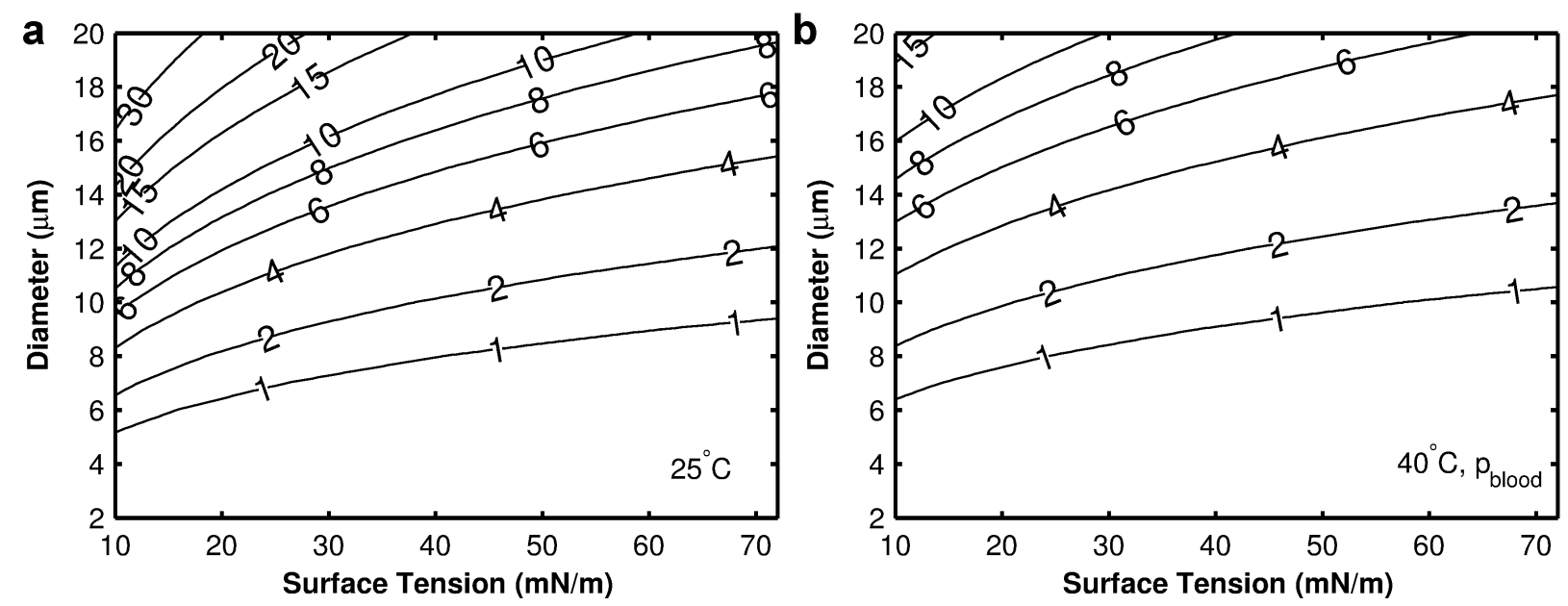

Figure 6.8: Microbubble lifetime in (a) water at $25^{\circ} \mathrm{C}$ and (b) blood at $40^{\circ} \mathrm{C}$. Isolines represent lifetime in seconds. Larger diameters and lower surface tensions increased lifetime. Lifetime decreased in blood compared to water.

nitrogen diffusion and partition due to the increase in temperature. Each factor was varied independently to determine the size of its response. At high surface tensions, the increased diffusion causes the greatest decrease in microbubble lifetime. At low surface tensions, the overpressure causes the greatest decrease in microbubble lifetime. This transition point varies with microbubble diameter, e.g. 20, 28, and $40 \mathrm{mN} / \mathrm{m}$ for 10,14 , and $20 \mu \mathrm{m}$ diameter microbubbles, respectively. The increased temperature decreases the Ostwald coefficient, which works to increase microbubble lifetime across all surface tensions. This increase, however, is not able to compensate for the decrease due to diffusion and blood overpressure.

\subsection{Discussion}

\subsubsection{In vivo imaging}

I present the first report of direct and immediate in-line intravascular administration of microbubbles produced from a microfluidic device. Several notable achievements were observed. First, no microbubble post-processing steps were necessary. The microbubbles did not have to be washed or concentrated prior to injection to achieve an acoustic signal. The 
excess shell material did not appear to affect the animal, probably a result of the shell being composed of only biocompatible materials (i.e. albumin and dextrose). Furthermore, the animals did not appear to become sensitized to the shell material as no allergic response was observed during the final imaging session. Second, microbubbles greater than $10 \mu \mathrm{m}$ in diameter were successfully injected without any gross adverse events to the animal. The safety profile of these larger microbubbles is probably related to the high solubility of the nitrogen gas used for the microbubble core. By quickly dissolving, the microbubbles have limited opportunities to coalesce, form gas emboli, and cause ischemic damage. Third, and most importantly, acoustic contrast was achieved despite production rates that are orders of magnitude lower than those achieved with agitation methods. Microfluidic production methods have long been plagued by low production rates when trying to produce stable microbubbles. These results suggest that by eliminating the need for stability, the production rate can be increased, and that it is not necessary to produce microbubbles at the same rate as agitation methods to achieve acoustic contrast.

While a strong acoustic signal was observed in the right ventricle, little to no signal was observed in the left ventricle when imaging with the microfluidic-produced microbubbles. This lack of contrast in the left ventricle is probably due to both the "sieve" effect in which the lung capillaries filter out large microbubbles [21], and increased dissolution rates as the microbubbles pass through the lungs $[162,103]$. As a result, few microbubbles survive the transit through the lung vasculature to provide contrast in the left ventricle. This behavior is not unexpected as these microbubbles were designed to be unstable by using highly soluble nitrogen gas and non-crosslinked albumin. Furthermore, passage through the lungs is not a requirement as the intended application of these microbubbles would be local production and use from a catheter tip, thus eliminating the need for systemic circulation.

Unlike the microfluidic-produced microbubbles, a strong signal was immediately observed in the left ventricle when the agitation-produced microbubbles were administered. The low solubility gas and the smaller mean diameter of the agitation-produced microbubbles likely 
enabled the microbubbles to survive the passage through the lungs and multiple passes through the circulation. While the agitation-produced microbubbles exhibited a higher intensity in the left ventricle, the image intensity in the right ventricle was only $70 \%$ of the image intensity achieved with the large diameter microfluidic-produced microbubbles.

In general, the contrast enhancement for both microfluidic-produced and agitationproduced microbubbles within the right ventricle followed a similar pattern, a sharp rise to peak intensity followed by a slower decrease back to baseline, with a median duration of double the injection time (Fig. 6.5). Two patterns for the image peak were also observed. The intensity curves either exhibited a single peak before decaying (Fig. 6.9) or a short plateau before signal decay. These results suggest that the microbubbles may have dispersed upon entering the bloodstream, causing a widening of the width of the intensity curve. The larger the dispersion of the microbubbles the wider the observed plateau. Interestingly, no correlation was observed between microbubble diameter or production rate and image intensity, rise time, decay time, or duration (Fig. 6.6). Given the high rate of gas dissolution out of the microfluidic-produced microbubbles, the distribution of microbubbles measured just prior to injection may not be representative of the distribution of microbubbles within the right ventricle, potentially obscuring any correlation between image intensity and microbubble diameter. This uncertainty of microbubble diameter within the vasculature also makes it difficult to directly compare simulation results with experimental results.

\subsubsection{Simulations}

\section{Oscillations}

A one-dimensional microbubble model was utilized to investigate the potential acoustic benefits of using larger microbubbles. Since the scattering cross section of microbubbles when insonated above their resonance frequency increases with the square of the diameter [90], increasing microbubble size should increase acoustic contrast. This regime is relevant as the predicted resonance frequency of a $10 \mu \mathrm{m}$ diameter microbubble is $0.6 \mathrm{MHz}$ [153], well 
below the imaging frequency in this study. While there are many 1-D numerical microbubble models [170], a model of an unencapsulated gas microbubble was chosen as the transient stability of the microfluidic-microbubbles suggests a weak shell, which would likely have a minimal effect on oscillations. In addition, we sought to analyze trends, rather than absolute values, thus minimizing the need for specific shell parameters. Frequencies between 4 and $15 \mathrm{MHz}$ were simulated as they cover the majority of clinical ultrasound frequencies. Microbubbles with diameters between 1 and $20 \mu \mathrm{m}$ were simulated as this represented the range of microbubbles found in clinical microbubble formulations as well as those used in this study.

Amongst all diameters, a peak displacement was observed only at an insonation frequency of $4 \mathrm{MHz}$, as the diameter of microbubbles in resonance at frequencies greater than $4 \mathrm{MHz}$ were less than $1 \mu \mathrm{m}$ (Fig. 6.7). Interestingly, despite minimal radial excursions, large microbubbles produced increasingly higher acoustic pressures in the simulated echo. Analysis of peak to peak pressure suggests that in certain specific cases, imaging of smaller microbubbles at resonance will produce greater contrast than the imaging of larger microbubbles. When using energy as a metric, however, the effect of resonance is abrogated as compared to the improvements achieved with larger microbubbles. Energy of the simulated acoustic signal is perhaps a better representation of the relationship between microbubble size, insonation frequency, and acoustic contrast since it takes into account the effect of the microbubble on the entire insonation pulse. In addition, tissue attenuation and multiple-scattering effects from imaging a population of microbubbles will likely further reduce resonance enhancement. Finally, an analysis of the frequency spectrum of the simulated echoes from microbubbles of various diameters when insonated at $12.5 \mathrm{MHz}$ shows that the magnitude of the fundamental frequency decreases with microbubble diameter. The smallest microbubble had the lowest energy in the fundamental frequency; however, as its resonance frequency is near $12.5 \mathrm{MHz}$, it had the largest superharmonic magnitude. 


\section{Dissolution}

Since the microbubble lifetime was difficult to measure, simulated dissolution times were analyzed to make inferences about the effect of surface tension and diameter on microbubble lifetime. Surface tension is the major contributor to microbubble dissolution since small microbubbles produce large Laplace pressures. The surface tension of a pure nitrogenwater interface is $71.4 \mathrm{mN} / \mathrm{m}$ [177]. The addition of albumin can reduce surface tension to $50 \mathrm{mM} / \mathrm{m}$ [178], while the addition of lipid surfactants can reduce surface tension to as low as $25 \mathrm{mN} / \mathrm{m}$ [179]. With a solid shell, such as lipid below its glass transition temperature, surface tension can be reduced to zero, resulting in microbubble lifetimes in excess of six hours [179]. If the microfluidic-produced microbubbles are considered to be fully coated with albumin, the simulations predict microbubbles with diameters less than $6 \mu \mathrm{m}$ would last less than 1 second, $14 \mu \mathrm{m}$ would last 4 seconds, and $20 \mu \mathrm{m}$ would last 12 seconds when placed in water at $25^{\circ} \mathrm{C}$ and 1 atm (Fig. 6.8a). These values are consistent with the unsuccessful attempts at measuring microbubble distribution using a Coulter counter which requires 30 seconds to acquire a measurement and previous work using an acoustic metric of microbubble half-life. For the acoustic metric, microfluidic-microbubbles are produced in a known volume and imaged with an ultrasound scanner until the image intensity is half the maximum intensity. Using this metric, microfluidic-produced microbubbles stabilized with albumin were measured to have a lifetime of $14 \mathrm{~s}$ as shown in section 4.4 . It should be noted that although microbubbles with no surfactant are predicted to have lifetimes upward of 8 seconds, microfluidic-produced microbubbles produced without a surfactant coalescence immediately upon formation within the microfluidic device.

When simulating microbubble lifetime in the blood, microbubbles less than $9 \mu \mathrm{m}$ were predicted to last less than 1 second, $14 \mu \mathrm{m}$ would last 3 seconds, and $20 \mu \mathrm{m}$ would last 7 seconds at $40^{\circ} \mathrm{C}$ and an overpressure of $4.3 \mathrm{kPa}$ (Fig. 6.8b). These results are consistent within an order of magnitude of the lifetimes observed in vivo with the microfluidic-microbubbles, which lasted an additional 10 seconds after the end of the infusion. For comparison, a 

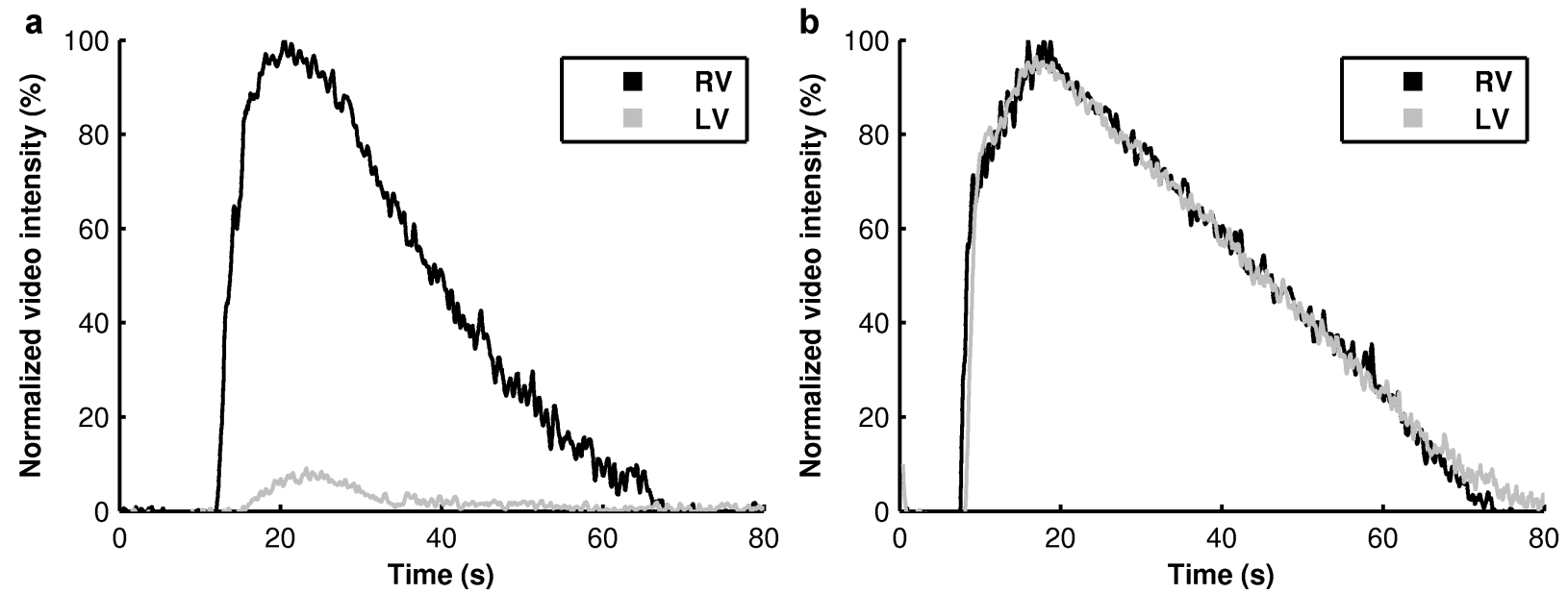

Figure 6.9: Representative B-mode image intensity in the right (RV) and left (LV) ventricle over time. Curves were normalized to the peak image intensity in the right ventricle. Rise time, duration, and decay time were calculated for the right ventricle only. (a) $14.4 \pm 1 \mu \mathrm{m}$ diameter microfluidic-produced microbubbles produced at a rate of 435,000 microbubble/s. Peak image intensity in the right ventricle was 108 a.u. (b) $5 \times 10^{6}$ agitation-produced microbubbles with a median microbubble diameter of $1.8 \mu \mathrm{m}$ diameter. Peak image intensity in the right ventricle was 65 a.u.

microbubble dissolution model for microbubbles composed of fluorocarbons (D) [103] predicts a lifetime in the bloodstream of 9.6 seconds for a $2 \mu \mathrm{m}$ diameter microbubble composed of octafluoropropane and stabilized with albumin. For a similar microbubble with a $14 \mu \mathrm{m}$ diameter, the predicted lifetime is 23 minutes. It should be noted that microbubbles composed purely of fluorocarbons are predicted to double in size before dissolving, whereas microbubbles composed purely of nitrogen are predicted to only shrink, thus providing an additional safety factor.

\subsubsection{Limitations}

As stated above, in the current implementation, the microfluidic-microbubbles are directly infused into the vasculature without any prior washing steps. While this production mode may be acceptable for general contrast or drug delivery applications, administering excess shell material into the vasculature may inhibit the ability to perform molecular imaging. To produce molecularly targeted microbubbles, the shell material must contains targeting ligands. 
In this case, the excess shell material may saturate the targeted ligands, thus preventing the necessary microbubble binding. Redesigned microfluidic devices that can filter the liquid phase post-microbubble fabrication $[180,181]$ may enable molecularly targeted microbubbles to be produced in situ.

Although no adverse events were observed, this may be due to the excellent ability of the lungs to filter gas from the bloodstream [20]. Consequently, additional studies will be required to determine whether transiently-stable microbubbles administered on the arterial side maintain the same safety profile as observed in the current study.

Finally, while the both the oscillation and dissolution simulations provide general trends, both models assume a single microbubble in an infinite medium. When the microbubbles are first produced and administered they are highly concentrated and, as a result, the single microbubble assumption is unlikely to be valid.

\subsection{Conclusion}

in this chapter, I describe the direct administration and ultrasound imaging of large diameter, transiently-stable, microfluidic-produced microbubbles. No acute harm to the animals was observed despite the large diameter of the microbubbles and co-administration of excess shell material with the microbubbles. Analysis of a one-dimensional model of microbubble oscillations suggests off-resonance insonation of large diameter microbubbles $(>10 \mu \mathrm{m})$ produces a larger acoustic response than insonation of small diameter microbubbles at resonance. In addition, analysis of a model of microbubble dissolution suggests lifetimes in vivo of less than 10 seconds, which was consistent with observed results. These observations provide additional support for a future paradigm whereby large transiently-stable microbubbles are fabricated in situ to provide enhanced image contrast and/or drug delivery. 


\section{Part II}

\section{ARFI and aaRBCs}




\section{Chapter 7}

\section{Assessing and improving acoustic}

\section{radiation force image quality using a 1.5D transducer design ${ }^{1}$}

\subsection{Abstract}

In this chapter, I present work on a numerical framework for the in silico development of a 1.5D transducer array designed to improve acoustic radiation force impulse (ARFI) imaging signal-to-noise ratio $\left(S N R_{A R F I}\right)$ and image contrast-to-noise relative to a conventional 1D linear array. Although this chapter focuses on ARFI imaging, radiation force in general has been shown to enhance microbubble imaging and drug delivery. Radiation force may be especially relevant for the transiently-stable in situ produced microbubbles described in Part 1 as it provides a means to quickly direct the microbubbles towards the vessel wall. As a result, a transducer optimized for ARFI should also be provide improved microbubble pushing. To predict performance gains from the proposed 1.5D transducer array, an analytical model for

${ }^{1}$ This chapter appears in the peer-reviewed publication:

A. H. Dhanaliwala, J. A. Hossack, and F. W. Mauldin, "Assessing and improving acoustic radiation force image quality using a 1.5-D transducer design," IEEE Trans Ultrason Ferroelectr Freq Control, vol. 59, no. 7, pp. 1602-1608, Jul. 2012. 
the upper bound of $S N R_{A R F I}$ was derived. The analytical model and 1.5D ARFI array were validated using a finite element model-based numerical simulation framework. The analytical model demonstrated good agreement with numerical results (correlation coefficient $=0.995)$, and simulated lesion images yielded a significant $(2.92 \mathrm{~dB} ; \mathrm{p}<0.001)$ improvement in contrast-to-noise ratio when rendered using the 1.5D ARFI array.

\section{$7.2 \quad$ Background}

Acoustic radiation force imaging is a classification of ultrasound imaging that uses tissue displacement, rather than acoustic reflectivity, as the source of image contrast. In general, these techniques produce images by using acoustic radiation force to push tissue, followed by a series of tracking pulses to measure displacement. This displacement can then be used to calculate relative or absolute stiffness. Radiation force imaging techniques, such as acoustic radiation force impulse (ARFI) imaging [182], shear wave imaging [183, 184, 185, 186, 187, 188], and monitored steady-state excitation and recovery imaging [189], have shown promise in detecting lesions in a variety of organs including the abdomen [190, 191], heart [192], liver [193], breast [194], and prostate [195] as well as measuring blood coagulation ex vivo [196, 197]. Since contrast is derived from stiffness, and stiffness is derived from displacement, the mean and variance of the displacement estimates have a large impact on lesion detection.

A major limiting factor in displacement estimation performance during ARFI imaging is echo decorrelation. While larger acoustic output, and thus larger acoustic radiation force, can increase ARFI image signal (i.e. displacement magnitude), displacement variance is also increased due to echo decorrelation from increased scatterer shearing [198, 199]. Additionally, increasing displacement magnitude is further limited by tissue ultrasound absorption and heating considerations (e.g. $<15 \mu \mathrm{m}$ for $<1{ }^{\circ} \mathrm{C}$ tissue heating [200]). In addition to increasing displacement variance, decorrelation derived from scatterer shearing is also associated with an underestimation of the peak displacement magnitude [199]. Therefore, displacement estimate 
improvements (i.e. high mean displacements and lower variance) are strongly dependent on the ability to reduce decorrelation between the reference and subsequent tracking echo signals. While signal processing methods, including principle component analysis [201, 202], can reduce decorrelation, it is also recognized that transducer design and imaging parameters effect the degree of differential motion across the point spread function, and thus, the observed levels of echo decorrelation [198]. In this paper, a new 1.5D ARFI transducer design is proposed for reduced echo decorrelation, and improved ARFI image signal-to-noise ratio $\left(S N R_{A R F I}\right)$, compared with a conventional 1D array. Echo decorrelation improvements are achieved using the $1.5 \mathrm{D}$ design because it enables electronic focusing in elevation and dedicated elements for each of the pushing and tracking functions. To investigate the relationship between echo decorrelation, transducer design, and image quality, I first develop an analytical model that relates the properties of a transducer to a radiation force image quality metric, $S N R_{A R F I}$. While the focus is on ARFI imaging, this analysis can be generalized to any radiation force imaging technique that share the common feature of deriving image contrast from displacement estimates. Using this new analytical model, an upper bound for $S N R_{A R F I}$ is derived as a function of tissue properties, transducer geometry, and imaging parameters. This model is validated using a numerical simulation framework and used, along with finite element model (FEM) simulations, to assess the performance of the proposed 1.5D array design compared with a convention $1 \mathrm{D}$ array in terms of $S N R_{A R F I}$ and contrast-to-noise ratio.

\subsection{Theory and Methods}

\subsubsection{Derivation of ARFI image SNR upper bound}

Image contrast in ARFI imaging is derived from differences in tissue stiffness, which are manifest in different displacement responses assuming constant acoustic radiation force excitation - i.e. assuming constant attenuation and tissue within a small distance of the 
transmit focus. With application of the same acoustic radiation force, stiffer tissue exhibits less displacement than more compliant tissue. Images are typically formed as maps of displacement estimates computed from locations within the 2D imaging region. To quantify image quality and assess the performance of the proposed 1.5D ARFI transducer design (described further below), an upper bound for $S N R_{A R F I}$ was derived. $S N R_{A R F I}$ can be defined as follows:

$$
S N R_{A R F I}=\frac{\mu_{d}}{\sigma_{d}}
$$

where $\mu_{d}$ is the mean and $\sigma_{d}$ is the standard deviation (or "jitter") of the estimated displacement, respectively. The standard deviation of ultrasound motion estimation depends on electronic signal-to-noise $\left(S N R_{E}\right)$, echo correlation $\left(\rho_{c o r}\right)$, center frequency $\left(f_{c}\right)$, motion estimator window length $(T)$, and fractional bandwidth $(B)$. An expression for the minimum jitter $\left(\hat{\sigma}_{d}\right)$ achievable from an unbiased time delay estimator was previously derived by Walker and Trahey [203]:

$$
\hat{\sigma}_{d} \geq \sqrt{\frac{3}{2 f_{c}^{3} \pi^{2} T\left(B^{3}+12 B\right)}\left(\frac{1}{\rho_{c o r}^{2}}\left(1+\frac{1}{S N R_{E}^{2}}\right)^{2}-1\right)} .
$$

While there are several parameters in (eqn. 7.2), echo correlation is an important factor dictating estimator performance since small increments result in large gains [203]. Under typical imaging conditions - $30 \mathrm{~dB} \mathrm{SNR}_{E}$ and $80 \%$ bandwidth - improving $\rho_{\text {cor }}$ by just 0.009 , from 0.99 to 0.999 , reduces jitter by $64 \%$.

The physical source of decorrelation $(1-\rho)$ between successive echoes result from either of two phenomena - out of beam motion or differential motion across the point spread function [201]. Both phenomena result in shifted and decorrelated versions of the reference echo, rather than perfectly shifted replicas, following displacement. As a result, bias and variance of the displacement estimate increase. Out of beam decorrelation typically occurs 
when scatterers move into and out of the point spread function between subsequent received echoes and is most common in imaging techniques with large displacements (e.g. elastography or Doppler) or in regions of the anatomy exhibiting large physiological motion (e.g. cardiac imaging). Differential motion decorrelation occurs when scatterers are differentially displaced within the pushing beam. Since displacements in ARFI and other radiation force imaging techniques are small $(\approx 10 \mu \mathrm{m}$ [200]), the contribution of out of beam motion to decorrelation is often viewed as insignificant and ignored in further theoretical or FEM analysis [198, 199, 204, 205]. However, as evidenced by the work of several groups to implement physiological motion filters [192, 206, 207], out of beam motion in ARFI imaging can occur due to physiological motion. Nevertheless, the present work continues as in previous work with contributions from out of beam decorrelation assumed to be negligible. This assumption is valid in nearly all physiological environments with the exceptions of cardiac imaging. Even in this environment, the source of out of beam decorrelation (i.e. cardiac motion) occurs on a time scale that is slower than the source of differential motion decorrelation (i.e. radiation force-induced displacements).

McAleavey et al [198] derived an expression for the magnitude of differential motion decorrelation in ARFI imaging that is a function of motion amplitude and the ratio of pushing beam width to tracking beam width. A major assumption of this derivation is that tissue displacement width is equal to the width of the two way response of the pushing beam and that displacement only occurs in the axial direction. Tissue, and other linearly elastic materials, however, exhibit mechanical coupling effects that result in tissue displacement widths that are wider than the pushing beam width and occur in all three directions (Fig. 7.1b) [204, 208]. To correct for this effect, echo correlation is described using the expression from McAleavey [198], but accounting for mechanical coupling through parameters, $L_{x}$ and $L_{y}$ :

$$
\rho_{\text {cor }}=1-\frac{1}{c^{2}} 4 A^{2} f_{c}^{2} \pi^{2} L_{x} L_{y}\left(\frac{1}{\sqrt{\left(1+L_{x}^{2}\right)\left(1+L_{y}^{2}\right)}}-\frac{4 L_{x} L y}{\left(1+2 L_{x}^{2}\right)\left(1+2 L_{y}^{2}\right)}\right)
$$




$$
L_{x}=\frac{D_{x}}{T_{x}}, L_{y}=\frac{D_{y}}{T_{y}}
$$

where $A$ is the maximum scatterer displacement, $c$ is the speed of sound, and $D_{x}, T_{x}, D_{y}$, $T_{y}$, are displacement and tracking beam widths in the lateral and elevational dimensions, respectively. The parameters relating displacement to tracking beam widths in (eqn. 7.3) and (eqn. 7.4), $L_{x}$ and $L_{y}$, replace the $W_{x}$ and $W_{y}$ parameters relating pushing to tracking beam widths from McAleavey [198]. Substituting (eqn. 7.3) into (eqn. 7.2) results in a new expression for the jitter lower bound in ARFI imaging:

$$
\begin{aligned}
& \hat{\sigma_{d}} \geq \\
& \sqrt{\left(\frac{\frac{3}{2 f_{c}^{3} \pi^{2} T\left(B^{3}+12 B\right)} *}{\left(1-\frac{1}{c^{2}} 4 A^{2} f_{c}^{2} \pi^{2} L_{x} L_{y}\left(\frac{1}{\sqrt{\left(1+L_{x}^{2}\right)\left(1+L_{y}^{2}\right)}}-\frac{4 L_{x} L_{y}}{\left(1+2 L_{x}^{2}\right)\left(1+2 L_{y}^{2}\right)}\right)\right)^{2}}\left(1+\frac{1}{S N R_{E}^{2}}\right)^{2}-1\right)},
\end{aligned}
$$

where $\hat{\sigma_{d}}$ is in units of seconds. McAleavey et al [198] further derived an expression for ARFI imaging signal (i.e. the expectation value of the displacement estimate). Replacing the assumption of equal displacement and pushing beam widths with the updated expression in (eqn. 7.4) results in a new expression for ARFI image signal:

$$
\mu_{d}=\frac{2 A L_{x} L_{y}}{\sqrt{\left(2 L_{x}^{2}+1\right)\left(2 L_{y}^{2}+1\right)}} .
$$


Substituting (eqn. 7.2) and (eqn. 7.6) into (eqn. 7.1) and scaling by $c / 2$ to maintain consistent units results in a final expression for the upper bound of $S N R_{A R F I}$ :

$$
\widehat{S N R}_{A R F I} \leq \frac{\mu_{d}}{\hat{\sigma}_{d} \frac{c}{2}}
$$

The upper bound in (eqn. 7.7) results in larger $S N R_{A R F I}$ values when compared with an upper bound derived using McAleavey's pushing beam width as a proxy for displacement width (Fig. 7.1).

\subsubsection{Numerical simulation framework}

A numerical simulation framework (Fig. 7.1a) was developed based on previously described finite element model (FEM) simulation methods [199, 204, 205] to validate the derived analytical model in (eqn. 7.7). Transducer design and relevant imaging parameters were selected and the intensity field was calculated using FIELD II [209]. Since intensity is proportional to the acoustic radiation force $[210,60]^{2}$, the FIELD II results were used to provide the body force input parameters for the FEM (COMSOL Multiphysics, COMSOL Inc. Burlington MA) simulations. FEM simulations were performed using a $50 \mathrm{~mm} \times 50 \mathrm{~mm} \times 50 \mathrm{~mm}$ block and a free tetrahedral mesh. Mesh element size was optimized to adequately sample displacement while minimizing the total number of elements. A maximum element size of $0.5 \mathrm{~mm}$ was used in regions where displacement estimates were extracted. To mimic human tissue using linear elastic assumptions, a Poisson's ratio $(\nu)$ of 0.49 [182] was used. Three-dimensional displacement fields were calculated from FEM simulations using a linear GMRES (generalized minimal residual) iterative solver [211] and a geometric multigrid preconditioner [212]. The

2 The acoustic radiation force can be calculated from:

$$
F \propto \frac{2 \alpha I}{c},
$$

where $F$ is the acoustic radiation force, $\alpha$ is the attenuation of the medium, and $I$ is the acoustic beam intensity [60] 
displacement results were then imported into MATLAB and scaled to achieve the desired peak displacement value. FEM simulations were conducted using static analysis where displacement fields represented immediate deformation responses to an impulsive acoustic radiation force. This time point is of greatest interest in ARFI imaging as images are typically formed using the maximum displacement parameter. Additionally, it is this time point where the proposed $1.5 \mathrm{D}$ array is anticipated to make the greatest contribution as later time points yield less differential motion decorrelation due to lower displacements and shear wave propagation [199]. Using these FEM methods, the mechanical coupling effects were incorporated into the proposed analytical model, $D_{x}$ and $D_{y}$ described above, by measuring the lateral full width half maximum (FWHM) displacement at the focus.

Received echo data from the proposed transducer array were simulated by first building a $20 \mathrm{~mm} \times 40 \mathrm{~mm} \times 25 \mathrm{~mm}$ volume comprising $2.5 \times 10^{5}$ uniformly distributed point scatterers with Gaussian distributed amplitudes. Reference, pre-motion, echoes were calculated using the FIELD II function calc_scat. A second echo, representing received echo data from the ARFI "tracking" pulse, was then simulated by displacing scatterers according to the FEM displacement field results and then repeating the calc_scat computation. Electronic noise was modeled as additive Gaussian white noise. Displacements were calculated using the Loupas autocorrelation method [213] and a sliding window length of 1.5 periods of the tracking center frequency.

$S N R_{A R F I}$ was calculated from displacement results as the ratio of the sample mean to the standard deviation of the calculated displacement. Mean and standard deviation for $S N R_{A R F I}$ values were computed by repeating the simulation over multiple $(\mathrm{n}=30)$ independent realizations of scatterer positions, amplitudes, and electronic noise.

\subsection{ARFI transducer design and comparison}

A 1.5D transducer array for ARFI imaging was designed for the purpose of increasing $S N R_{A R F I}$ using insights from the analytical model, while taking hardware complexity into 


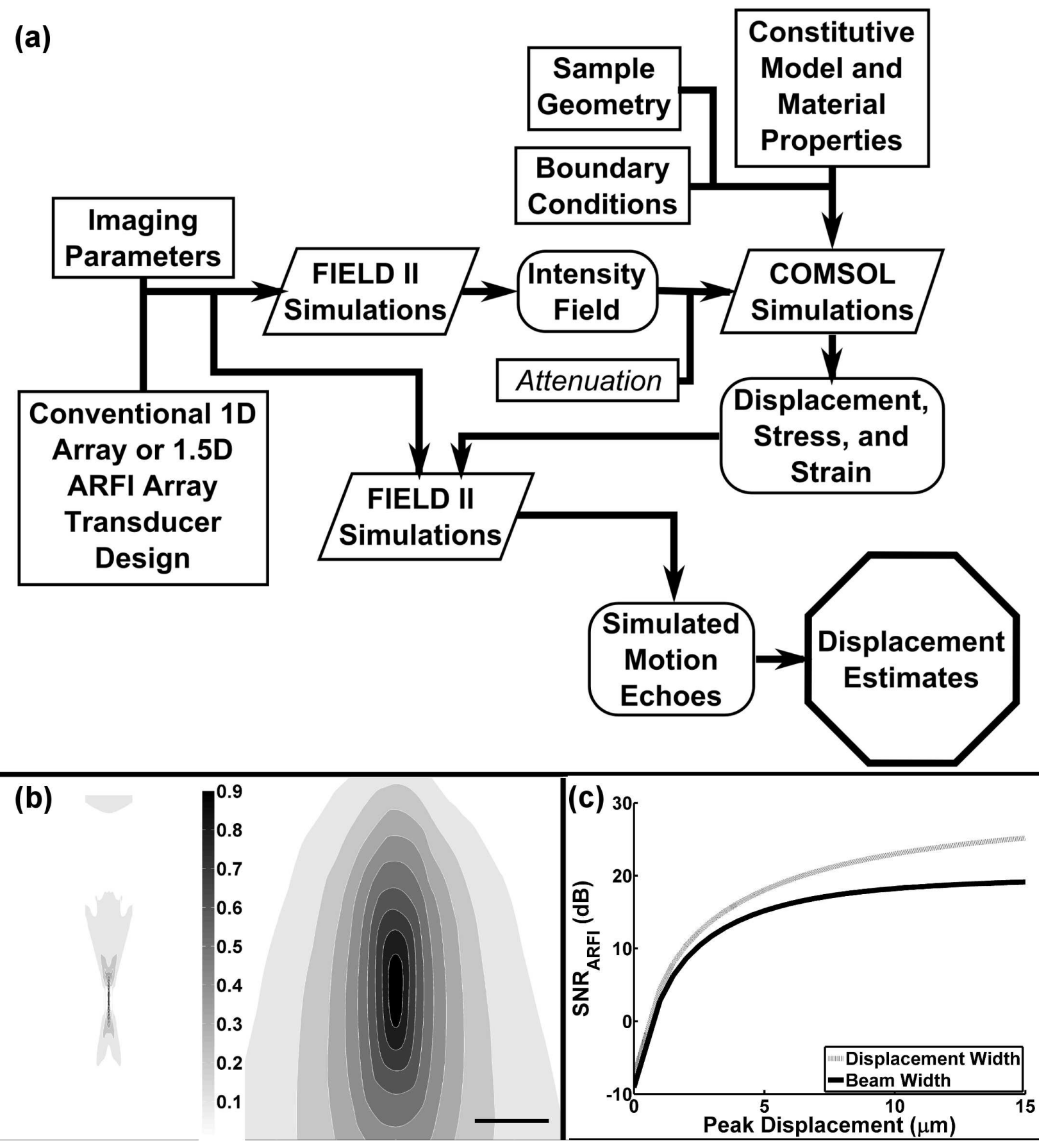

Figure 7.1: (a) Block diagram of the numerical simulation framework. (b) Intensity field output from FIELD II (c) Displacement output from tissue FEM. Scale bar and color map of relative intensity apply to both images. (d) Acoustic radiation force impulse SNR upper bound from (eqn. 7.7) when using the actual displacement width parameter or the beam width proxy as in McAleavey et al [198]. 

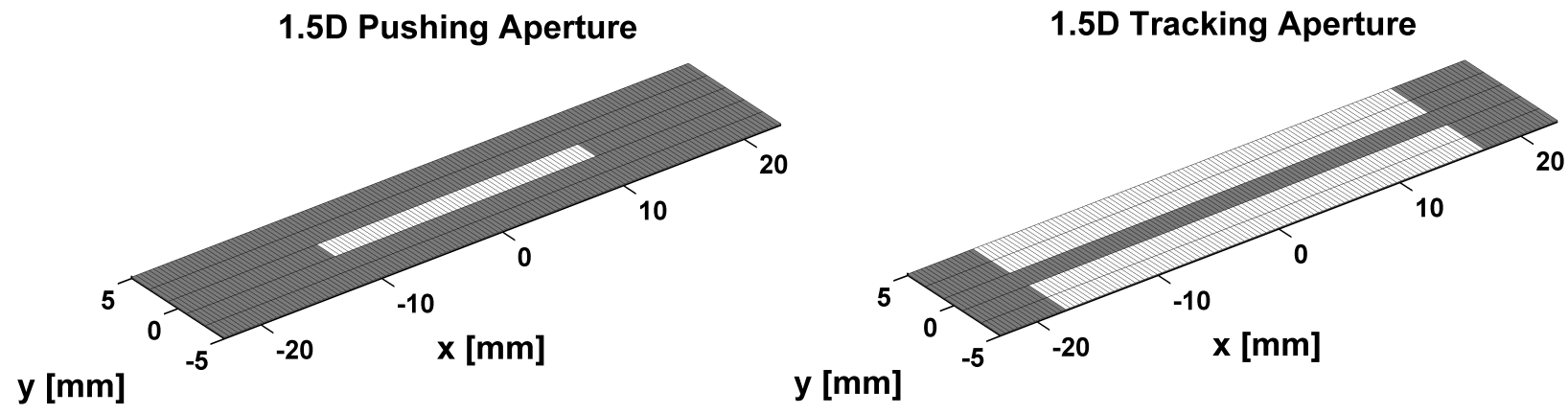

Figure 7.2: Pushing and tracking array geometry for the proposed 1.5D ARFI imaging transducer. White denotes active elements in the array.

Table 7.1: Simulation parameters for the conventional 1D array and 1.5D ARFI array

\begin{tabular}{ccccc}
\hline & \multicolumn{2}{c}{ Conventional 1D } & \multicolumn{2}{c}{ 1.5D ARFI } \\
& Pushing & Tracking & Pushing & Tracking \\
\hline \hline Elements & 60 & 96 & 60 & $4 \times 96$ \\
Center frequency $(\mathrm{MHz})$ & 4.21 & 6.15 & 2 & 7 \\
Bandwidth $(\%)$ & 50 & 80 & 50 & 80 \\
Lateral pitch $(\mu \mathrm{m})$ & & 350 & 1 \\
Elevation pitch $(\mathrm{mm})$ & & \multicolumn{3}{c}{} \\
\hline With the exception of number of elements and center frequency, all \\
other values were constant between the two designs in order to better \\
compare the improvement in $S N R_{A R F I}$ due to increased pushing to \\
tracking beam width ratios.
\end{tabular}

consideration (i.e. $1.5 \mathrm{D}$ is less complex than a $1.75 \mathrm{D}$ or $2 \mathrm{D}$ array solution). $S N R_{A R F I}$ improvements using the $1.5 \mathrm{D}$ array is derived from mitigation of echo decorrelation through two primary mechanisms. First, the additional rows of elements enable electronic focusing in elevation, thus reducing the tracking beam width in this dimension. Second, the functions of pushing and tracking pulses can be assigned to different rows (Fig. 7.2). This allows pushing rows to use lower frequency elements than the tracking rows, thus widening the pushing beam width relative to the tracking beam width. The parameters describing the $1.5 \mathrm{D}$ array and a conventional 1D array are given in Table 7.1.

$S N R_{A R F I}$ for the $1.5 \mathrm{D}$ ARFI array and a conventional $1 \mathrm{D}$ array were computed over a range of peak displacements, using the numerical framework outlined above. In addition, a $4 \mathrm{~mm}$ diameter soft lesion [214] at a depth of $20 \mathrm{~mm}$ within a stiffer homogeneous background 
tissue, representing breast tissue [215], was simulated. All parameters were the same as above except the maximum element size of the tissue block, transducer focal area, and lesion were $2 \mathrm{~mm}, 0.5 \mathrm{~mm}$, and $0.25 \mathrm{~mm}$, respectively. Images of the lesion produced with the 1.5D ARFI or the conventional 1D array were produced. Elastic modulus contrast between the simulated lesion and background tissue was 1:10 (e.g. $10 \mathrm{kPa}$ lesion in a $100 \mathrm{kPa}$ background) with peak displacement inside the lesion of $12 \mu \mathrm{m}$. Image contrast-to-noise ratio (CNR) was calculated as [216]:

$$
C N R=20 \log _{10}\left(\frac{\left|\mu_{i}-\mu_{o}\right|}{\sigma_{o}}\right)
$$

on median filtered images, where $\mu_{i}$ and $\mu_{o}$ are the mean displacements within equal area regions inside and outside the lesion, respectively, and $\sigma_{o}$ is the standard deviation of displacement outside the lesion.

\subsection{Results and discussion}

To validate the analytical model (eqn. 7.7), $S N R_{A R F I}$ was calculated for independent realizations of scatterers with increasing peak displacement for both the conventional 1D array and 1.5D ARFI array (Fig. 7.3). The solid lines denote the derived $S N R_{A R F I}$ upper bound and the symbols represent numerical simulation results. Excellent agreement was observed between numerical simulation results and the proposed SNRARFI analytical upper bounds - correlation coefficients of 0.995 and 0.998 for the $1 \mathrm{D}$ and $1.5 \mathrm{D}$ arrays respectively. In addition, the 1.5D ARFI array had an increased $S N R_{A R F I}$ for all normalized displacements as compared to the $1 \mathrm{D}$ array $(\mathrm{p}<0.05)$. Finally, when $S N R_{A R F I}$ was calculated for increasing $S N R_{E}$, it was found to plateau above $30 \mathrm{~dB}$. This trend substantiates the importance of improving $S N R_{A R F I}$ through reductions in echo decorrelation as corresponding $S N R_{A R F I}$ gains achieved via higher $S N R_{E}$ are negligible. 
Table 7.2: Tissue displacement width, tracking beam width and the ratio of displacement width to tracking width (Displ, Track, Displ:Track, respectively) for the 1.5D ARFI and the conventional 1D array.

\begin{tabular}{c|ccccc}
\hline Transducer & Direction & Displ $(\mathrm{mm})$ & Track $(\mathrm{mm})$ & Displ:Track $\left(D_{i}\right)$ & Push:Track \\
\hline \hline Conventional 1D & Lateral & 6.4 & 0.25 & 25.9 & 1.5 \\
& Elevation & 10.3 & 5.4 & 1.9 & 1.2 \\
\multirow{3}{*}{ 1.5D ARFI } & Lateral & 8.1 & 0.25 & 32.9 & 2.9 \\
& Elevation & 17.7 & 1 & 17.8 & 13.2 \\
\hline
\end{tabular}

The 1.5D ARFI array has a 9-fold increase in $D_{y}$ and a 1.2-fold increase in $D_{x}$ as compared to the conventional 1D array. For comparison, the ratio of pushing beam width to tracking beam width is also included.

Pushing and tracking beam widths for the conventional 1D and 1.5D ARFI imaging arrays were calculated using FIELD II (Table 7.2). By enabling electronic focusing in the elevational dimension, the 1.5D ARFI array possessed a significantly wider pushing beam and narrower tracking beam width as compared to the conventional 1D array. Additionally, due to an ability to use dedicated elements for pushing and tracking functions, different center frequency ceramics were used for different rows of the 1.5D array (e.g. $2 \mathrm{MHz}$ and $7 \mathrm{MHz}$ for the 1.5D array versus 4.21 MHz and 6.15 MHz for the conventional 1D array as described in Table 7.1). Thus, while more substantial gains in displacement to tracking beam widths were observed in the elevational dimension, gains were also achieved in the lateral dimension due to a larger range of center frequencies.

Lesion simulations were performed with independent realizations of scatterers for the purpose of illustrating the effects of transducer geometries and parameters on image quality (Fig. 7.4). The lesion was better delineated - a $2.92 \mathrm{~dB}$ improvement (two-sided t-test, $\mathrm{p}<0.0001)$ - when imaged using the 1.5D ARFI array over the conventional 1D array (Table 7.3).

As stated earlier, while the focus of this chapter was towards the development of an ARFI imaging optimized transducer, transducers optimized to produce radiation forces also have a role in microbubble imaging. As described in section 1.4 of the introduction, radiation force has been shown to improve microbubble imaging and microbubble enhanced drug 


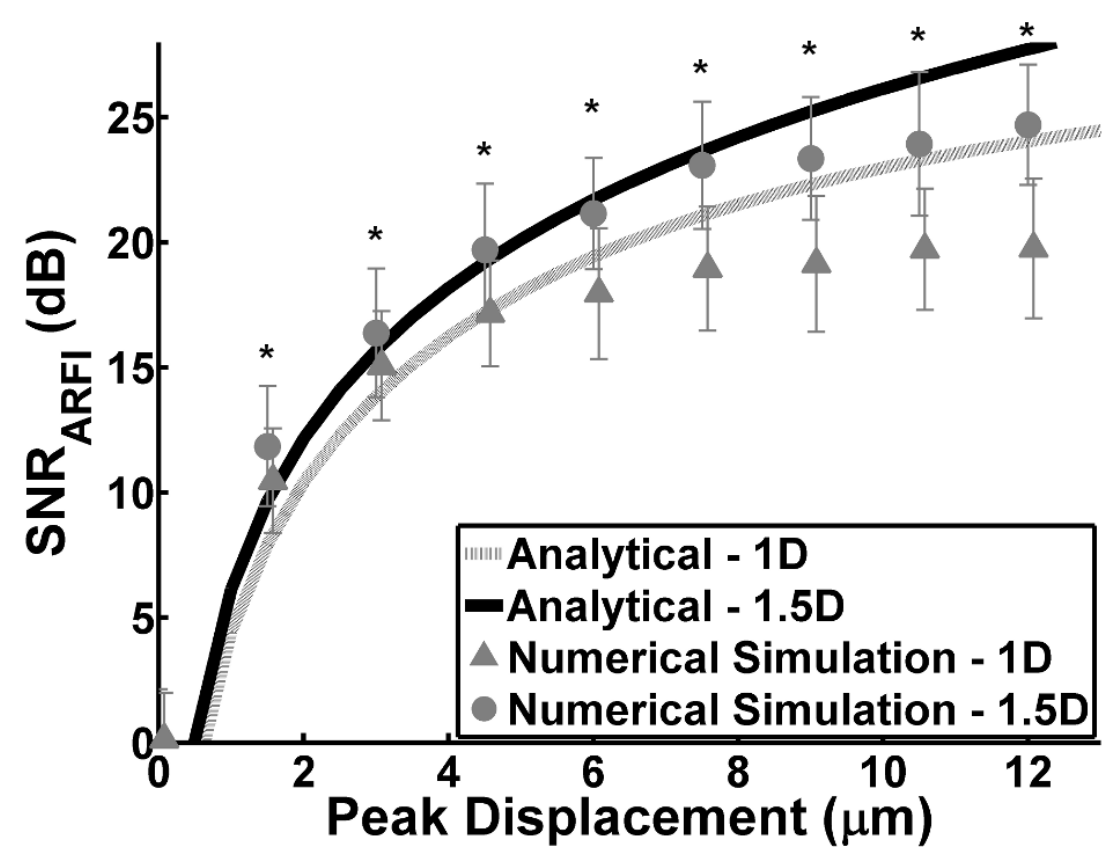

Figure 7.3: $S N R_{A R F I}$ as a function of peak displacement. Solid lines represent the derived analytical model and the symbols with error bars are numerical simulation results for the $1 \mathrm{D}$ and 1.5D arrays. $S N R_{E}=30 \mathrm{~dB}$. Mean and standard deviations for $S N R_{A R F I}$ were calculated over 30 independent runs. ${ }^{*}$ indicates significant difference between $1.5 \mathrm{D}$ and $1 \mathrm{D}$ numerical simulation (two sided t-test $\mathrm{p}<0.05)$.

Table 7.3: CNR and SNR values of the simulated lesion when imaged with the conventional 1D and the $1.5 \mathrm{D}$ ARFI array

\begin{tabular}{lccc}
\hline Transducer & CNR $(\mathrm{dB})$ & SNR $_{\text {lesion }}(\mathrm{dB})$ & $\mathrm{SNR}_{\text {tissue }}(\mathrm{dB})$ \\
\hline \hline Conventional 1D & $6.91 \pm 1.21$ & $7.29 \pm 0.62$ & $14.48 \pm 0.97$ \\
1.5D ARFI & $9.82 \pm 0.83$ & $11.02 \pm 0.68$ & $23.26 \pm 1.64$ \\
\hline A 2.92 dB average improvement in CNR was achieved with the 1.5D \\
ARFI array (two-sided t-test, $\mathrm{p}<0.0001, \mathrm{n}=30)$. The average SNR \\
values inside and outside the lesion were also significantly improved \\
with the 1.5D ARFI array $(\mathrm{p}<0.0001)$.
\end{tabular}

delivery by concentrating microbubbles out of flow and towards the vessel wall. For in situ produced microbubbles, this may be especially important as radiation force could help push microbubbles away from the microfluidic output and towards the vessel wall. Consequently, the designed ARFI imaging transducer may also enable enhanced imaging and drug delivery when paired with in situ microbubble production. Furthermore Kilroy et al [217, 102], using a different but conceptual similar simulation framework for transducer design, developed 


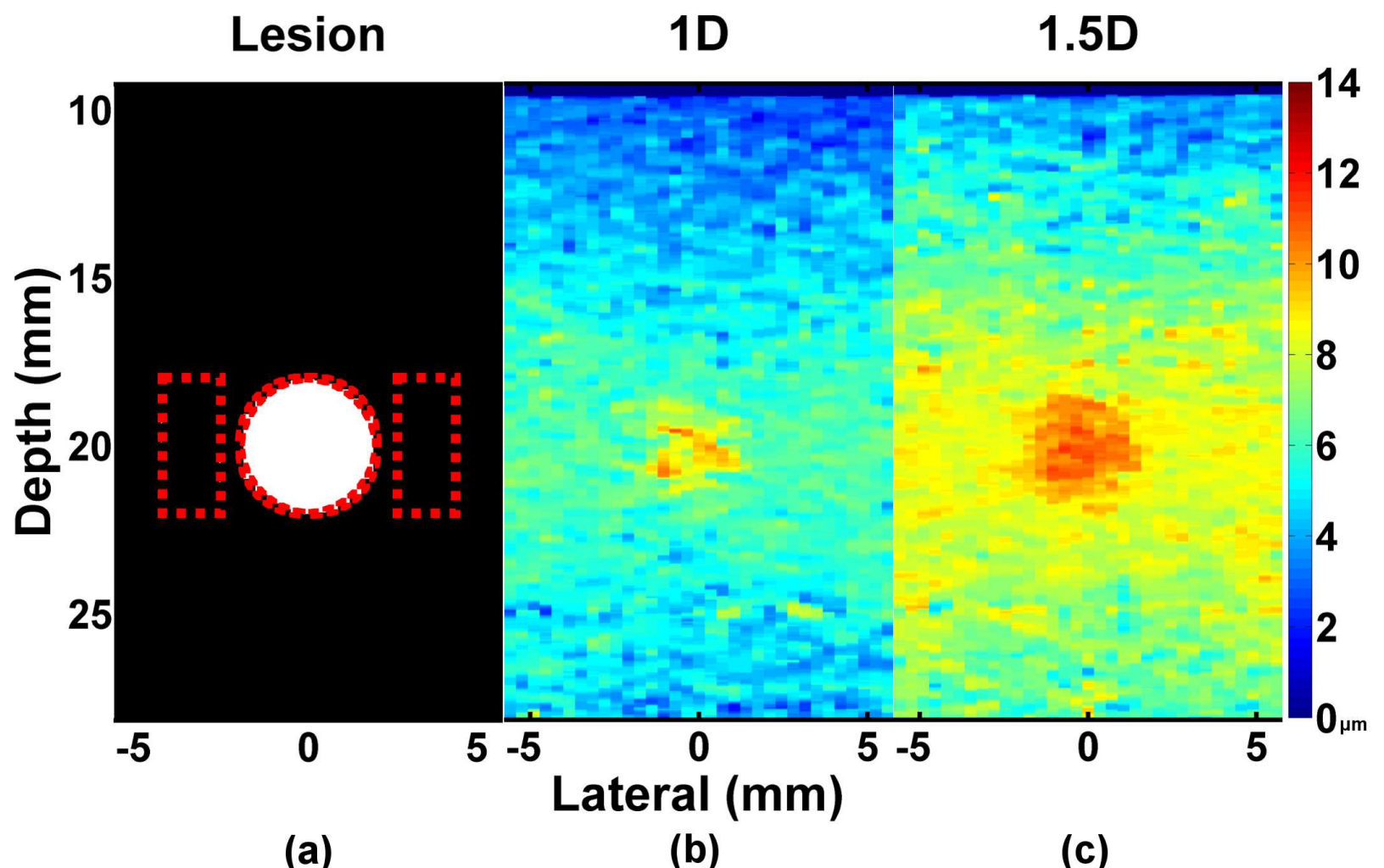

Figure 7.4: Simulated image of a compliant lesion embedded in stiffer surrounding tissue (1:10 ratio in Young's Modulus) imaged using either the (b) 1D or the (c) 1.5D array. The 1.5D array exhibited a $2.92 \mathrm{~dB}$ average improvement in CNR over the conventional $1 \mathrm{D}$ array $(\mathrm{n}=30)$. The (a) black and white image illustrates the location and size of the lesion (4 $\mathrm{mm}$ diameter, $20 \mathrm{~mm}$ depth) and the dotted lines indicate regions of interest (ROI) used for CNR and SNR calculations.

an IVUS catheter optimized for radiation force. These results further validate the concept of a numerical framework for transducer design, as well as move us closer to the desired implementation of in situ microbubble production coupled to an intravascular ultrasound (IVUS) transducer to produce a multifunction IVUS catheter.

\subsection{Conclusion}

In this chapter, an analytical model for the upper bound of ARFI image SNR, a metric for comparing ARFI image quality, was derived as a function of tissue properties, imaging parameters, and transducer geometry. The analytical model was validated using a numerical simulation framework that coupled FIELD II to a finite element tissue model. By accounting 
for tissue displacement, there was excellent agreement between the analytical model and the numerical simulations. Using insight gained from the analytical model, a novel 1.5D array for ARFI imaging was designed. The 1.5D ARFI array increased displacement to tracking beam width ratio by allowing electronic focusing in the elevation and by allowing the pushing elements to be at a lower center frequency than the tracking elements. These improvements, impossible in a 1D array, resulted in an overall increase in echo correlation and improved $S N R_{A R F I}$ as predicted by the analytical model. When compared to a conventional 1D array, the 1.5D ARFI array provided significantly improved displacement estimation, ARFI image SNR, and image CNR. Finally, the coupling of transducer design to an accurate analytical model of ARFI image SNR highlights the potential utility of this new tool. By using the analytical model to develop more sophisticated transducers that improve image quality, the clinical utility of ARFI can be improved for more accurate assessments of tissue material properties or better delineation of different tissue structures, such as lesions or arterial plaques. 


\section{Chapter 8}

\section{Acoustically active red blood cells as an acoustically triggered drug delivery vehicle}

\subsection{Abstract}

Microbubbles can improve ultrasound image contrast as well as enhance drug delivery. Current microbubble formulations, however, have a limited therapeutic carrying capacity. Red blood cells continue to be investigated as drug delivery vehicles given their large carrying capacity and inherent biocompatibility. Individual red blood cells, however, cannot be easily tracked in vivo and drug release from a drug-loaded red blood cell is difficult to trigger specifically in space or time. In this chapter, I describe work to combine the benefits of microbubbles with the benefits of red blood cells by loading microbubbles into red blood cells towards the ultimate goal of producing acoustically active red blood cells (aaRBCs). Methods to load stable lipid-shelled microbubbles and nanometer-sized microbubble precursors into red blood cells are described. Smaller microbubble precursors are investigated in order to improve red blood cell loading efficiency over micrometer sized microbubbles. Once loaded, 
the microbubble precursors can be converted to a gaseous microbubble. aaRBCs have the potential to be a long-lived, biocompatible ultrasound contrast agents that have a large therapeutic carrying capacity and an acoustically triggered release mechanism.

\subsection{Background}

Microbubbles have several limitations as drug delivery vehicles. First, microbubbles have a short lifetime in vivo. Once injected, microbubble clearance can occur as quickly as five minutes after administration [23]. This short lifetime can make ligand-receptor targeting difficult since any given microbubble only has a few passes through the vasculature to find and bind its target. Second, microbubbles have a limited therapeutic payload. For small molecule therapeutics loaded within the shell, the microbubble shell layer has a limited volume - a $1 \mu \mathrm{m}$ diameter microbubble with a $10 \mathrm{~nm}$ thick shell has a total volume of $0.1 \mathrm{fL}$. The shell could be made thicker in order to increase loading capacity [218]; however, this method is only compatible with hydrophobic therapeutics. Plasmids for gene therapy can be attached electrostatically to the shell, but this requires positively charged lipids that have biocompatibility issues. In addition, total payload is limited by small shell surface area. Finally, drug-containing liposomes attached to the microbubble shell [219, 220] have shown promise as a means to increase payload. In this chapter, however, I propose a different approach that utilizes red blood cells to increase therapeutic payload.

Red blood cells are similar in size to microbubbles, are produced at a rate of two million per second, and continue to be investigated as a platform for drug, gene, and enzyme delivery [221, 222, 223, 224]. Red blood cells are ideal drug delivery vehicles as they are inherently biocompatible, have a half-life of up to a month [225, 226], and have a large therapeutic carrying capacity — a human red blood cell has an average internal volume of $90 \mathrm{fL}$. In the most common implementation for preparing red blood cells as drug delivery vehicles, red blood cells are lysed then resealed in a hypotonic solution in the presence of 
the desired therapeutic. This procedure opens pores within the red blood cell [227, 228, 229] resulting in hemoglobin diffusion out of, and small molecule diffusion in to, the red blood cell. The loss of hemoglobin causes the red blood cells to become pale, hence the term "red blood cell ghosts". Targeting, or sensitizing moieties, can be added to the cell membrane to enhance targeting or provide a photo or pH sensitive trigger for therapeutic release [230].

Red blood cell ghosts as a drug delivery vehicle have their own limitations. Once administered, red blood cell ghosts are difficult to track in real-time in vivo. Unlike microbubbles, at clinical ultrasound imaging frequencies $(6-15 \mathrm{MHz})$, red blood cells do not provide sufficient acoustic impedance mismatch and are therefore effectively invisible to ultrasound [231]. Tracking via loading of radiolabeled-tracers has been investigated [232], but this method is more appropriate for pre-clinical studies. Drug release from red blood cell ghosts is also difficult to trigger. Light has limited depth penetration and $\mathrm{pH}$ is difficult to control in vivo. As a result, therapeutic release is dictated by diffusion out of the red blood cell ghost.

In this chapter, I propose a new drug delivery agent, termed acoustically active red blood cell ghosts (aaRBCs), that combines the advantages of microbubbles to offset the limitations of red blood cell ghosts and vice versa. In this framework, microbubbles or microbubble precursors are incorporated into red blood cells to produce aaRBCs. The microbubbles inside the red blood cell should enable ultrasound tracking of the red blood cells, as well as provide an acoustic trigger for release of the loaded therapeutic. The red blood cell should provide a biocompatible membrane to protect the microbubble and increase circulation time, as well as a larger therapeutic carrying capacity. Production methods are outlined as well as some initial characterization. 


\subsection{Materials and Methods}

\subsubsection{Microbubble fabrication}

Stable perfluorocarbon, lipid-shelled microbubbles were prepared as described in [145]. 1,2dipalmitoyl-sn-glycero-3-phosphocholine (DPPC, Avanti Polar Lipids, Alabaster, AL) and polyethylene glycol stearate (PEG40st, Sigma-Aldrich, St. Louis, MO), both at $2 \mathrm{mg} / \mathrm{mL}$ in isotonic saline, were sonicated (XL2020, Misonix Inc., Farmingdale, NY) to generate an aqueous micellar dispersion. Decafluorobutane gas (DFB, $\mathrm{C}_{4} \mathrm{~F}_{10}$, Scott Medical Products, Plumsteadville PA) was then sparged through the aqueous phase while the solution was sonicated at $600 \mathrm{~W}$ to create a microbubble dispersion. The microbubbles were floated overnight to separate the microbubbles from the excess lipid. The microbubble cake was removed and stored under a decafluorobutane atmosphere, in sealed vials, at $4^{\circ} \mathrm{C}$. The fluorophore 1,1'-dioctadecyl-3,3,3',3' tetramethylindocarbocyanine perchlorate (DiI, Ex/Em 549/565,Molecular Probes, Eugene, OR, USA) was added at a 1\% molar ratio of DiI:DPPC to the aqueous phase prior to sonication to enable fluorescence microscopy of the microbubbles. A Coulter counter (Multisizer III, Beckman Coulter, Brea, CA) was used to size and count the microbubbles.

\subsubsection{Microbubble precursor fabrication}

Liquid fluorocarbon nanodroplets were fabricated by sonication of liquefied DFB. 4 mL of either deionized water alone or, solutions of $5 \mathrm{mg} / \mathrm{mL}$ bovine serum albumin (BSA, SigmaAldrich, St. Louis, MO), 2\% Tween 20 (Fisher-Scientific, Pittsburgh, PA), or 1\% Zonyl ${ }^{1}$

\footnotetext{
${ }^{1}$ An approximation for the amount of Zonyl necessary to sufficiently coat particles of a specific size was calculated from:

$$
V_{Z}=\frac{3 V_{g} \rho_{g} M W_{Z}}{r_{d r o p} \rho_{L} S A_{Z} N_{A} S_{Z}}
$$

where $V_{Z}$ is the volume of zonyl necessary to coat the number of $r_{d r o p}$ radius microbubble precursors produced from an initial $V_{g}$ volume of gas. $\rho_{g}$ is the density of the gas, $\rho_{L}$ is the density of the liquid, $M W_{Z}$ is the molecular weight of zonyl (see E), $S A_{Z}$ is the surface area of a single zonyl molecule estimated at $0.3 \mathrm{~nm}^{2}$ [233], $N_{A}$ is Avogadro's number, and $S_{Z}$ is the stock concentration of Zonyl.
} 
(FS-300, Sigma-Aldrich, St. Louis, MO) in deionized water was added to a $10 \mathrm{~mL}$ syringe. The air was replaced with $6 \mathrm{~mL}$ of $\mathrm{DFB}$ and the syringe stoppered. The syringe was then compressed to convert the gaseous DFB into a liquid state. The pressurized syringe was placed in a bath sonicator (2510, Branson, Danbury, CT) with ice-chilled water. Every attempt was made to keep the position of the syringe and the volume of the water identical between runs. The syringe was sonicated in 10 minute increments for a total of 20 minutes. After each increment, the water was re-chilled in an ice bath prior to restarting sonication. Successful nanodroplet production was qualitatively determined by observing an increase in cloudiness within the syringe and the loss of large $(\approx \mathrm{mm}$ sized $)$ liquid DFB droplets. After sonication, the solution was a uniform white.

The nanodroplets were size sorted via differential centrifugation ${ }^{2}$. A 60 s spin at 5,000 RCF in a $2 \mathrm{~mL}$ eppendorf pelleted all particles larger than $500 \mathrm{~nm}$ in diameter. The supernatant was collected then spun at 16,000 RCF for 5 minutes to pellet all remaining particles. A Coulter counter was used to size particles larger than $1 \mu \mathrm{m}$ in diameter. Dynamic light scattering (Nano ZS90, Malvern Instruments Inc., Westborough, MA) and nanoparticle tracking analysis (Nanosight, Malvern Instruments Inc., Westborough, MA) were used to size particles less than $1 \mu \mathrm{m}$.

\subsubsection{Acoustically-active red blood cell production}

Acoustically-active red blood cell ghosts (aaRBCs) were produced using a modified dilution lysis method [227, 232, 234, 229] (Fig. 8.1). Human blood from the University of Virginia clinical laboratory (IRB waiver on file), collected in $1.8 \mathrm{mg} / \mathrm{mL}$ ethylenediaminetetraacetic

${ }^{2}$ The centrifugation speeds and times were determined empirically, but were guided by the equation for settling velocity:

$$
S V_{d r o p}=\frac{2 g X r^{2}\left(\rho_{d r o p}-\rho_{L}\right)}{9 \eta_{L}}
$$

where $S C_{d r o p}$ is the settling velocity of the particle, $g$ is gravitational acceleration, $X$ is the gravitational multiplier (i.e. RCF), $r_{d r o p}$ is the microbubble precursor radius, $\rho_{\text {drop }}$ is the density of the microbubble precursor, $\rho_{L}$ is the density of the surrounding liquid, and $\eta_{L}$ is the viscosity of the surrounding liquid. 


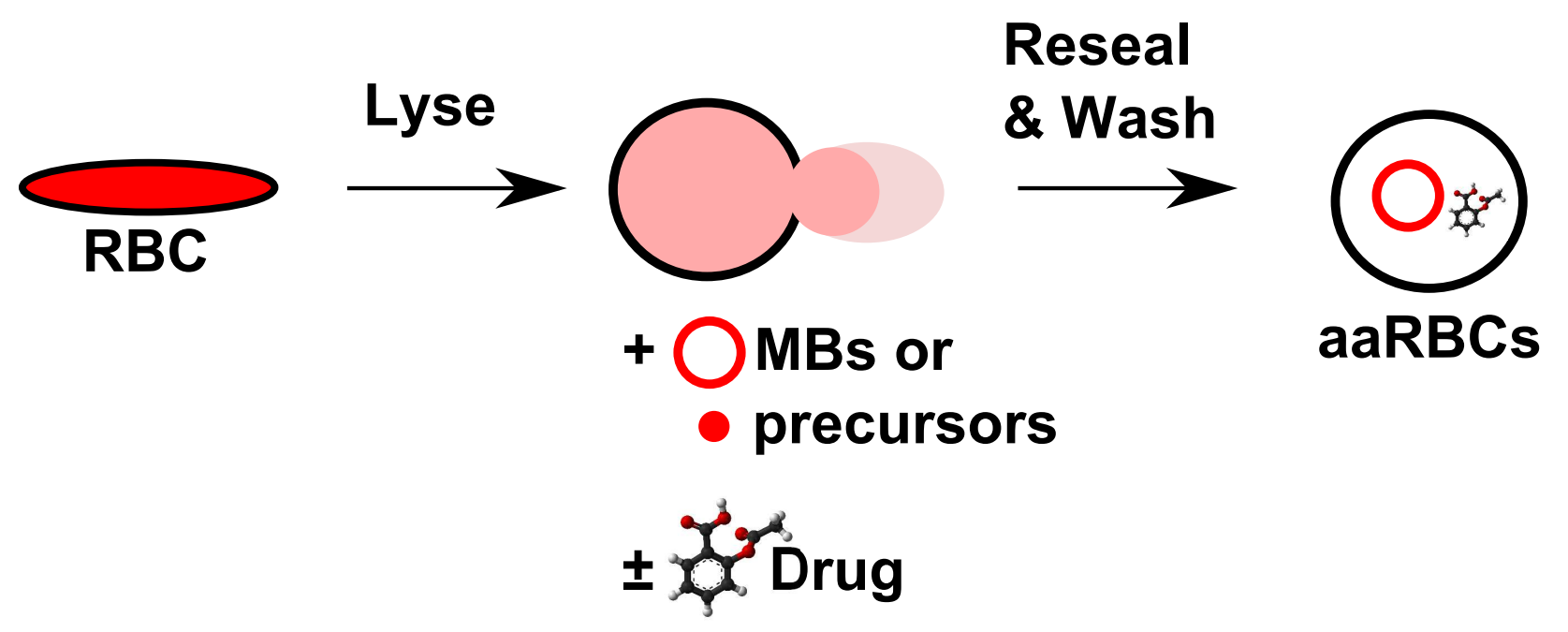

Figure 8.1: Procedure for loading microbubbles or microbubble precursors into red blood cells. Washed red blood cells less than 7 days old are placed in a hypotonic solution containing microbubbles or microbubble precursors. Concentrated saline is then added to restore isotonicity, and to facilitate membrane resealing. The cells are washed to remove unencapsulated microbubbles or microbubble precursors. Resulting particles are termed acoustically active red blood cell ghosts (aaRBCs).

acid (EDTA, Sigma Aldrich, St. Louis, MO) to prevent clotting, was acquired and used within 7 days. The whole blood was washed three times in isotonic saline, removing the plasma and buffy coat, then stored at $4{ }^{\circ} \mathrm{C}$. One vol. of packed cells were first pre-lysed in 9 vol. $0.25 \mathrm{x}$ phosphate buffered saline with calcium and magnesium $(\mathrm{PBS}+/+$, Fisher-Scientific, Pittsburgh, PA). The cells were incubated for 5 minutes on ice, spun at 10,000 RCF for 5 minutes and then lysed again. To load the particles, 1 vol. of the pre-lysed cells was added to 40 vol. of the lysing solution consisting of $0.01 \mathrm{x}$ PBS without calcium and magnesium, $100 \mu \mathrm{M}$ EDTA, and the particles to be loaded. The cells were incubated on a rocker, overnight at $4^{\circ} \mathrm{C}$. If loading microbubble precursors, the solution was kept under pressure during the overnight incubation. After the incubation, a sufficient quantity of a concentrated salt solution plus a magnesium solution (e.g. $60 \mu \mathrm{L}$ of a $5 \mathrm{M} \mathrm{NaCl}$ solution plus $10 \mu \mathrm{L} 1 \mathrm{M} \mathrm{MgSO}_{4}$ ) was added to restore isotonicity. Cells were incubated for an additional hour at $25^{\circ}$ to facilitate membrane resealing. The red blood cell ghosts were then washed in isotonic saline until the supernatant was free of unencapsulated particles. Control red blood cell ghosts were produced in a similar fashion by omitting the particles from the lysing media. 
The aaRBCs were imaged using phase contrast and fluorescence microscopy to confirm loading. Since the microbubble precursors do not fluoresce, $200 \mathrm{~nm}$ diameter fluorescent polystyrene beads were loaded as a surrogate marker. A Coulter counter (Z2 particle sizer, Beckman Coulter Brea, CA) was used to measure concentration and size of the red blood cells pre- and post-loading.

\subsubsection{Microbubble Survival}

Given the number of steps requiring centrifugation, the effect of centrifugation speed on microbubble longevity measured. Microbubbles in saline were centrifuged at 1,000x, 3,000x, and 10,000x relative centrifugation force $(\mathrm{RCF})$ and the concentration was measured with a Coulter counter (Multisizer III, Beckman Coulter Brea, CA) every 20 minutes. The concentrations were normalized to the initial pre-centrifugation microbubble concentration and a curve of best fit that maximized $\mathrm{R}^{2}$ was calculated.

\subsubsection{Acoustic conversion of microbubble precursors}

A small animal high frequency ultrasound scanner (Vevo 2100, VisualSonics, Toronto, ON, CA) was used to investigate the pressure and frequency combinations sufficient to convert the microbubble precursors into microbubbles. A high-walled petri dish filled with gas saturated water was placed on an inverted microscope. The face of the ultrasound transducer was then placed into the water from an angle on the side to prevent interference with the microscope light source. The optical focus of the microscope was aligned with the acoustic focus of the transducer by visualizing a needle in both the B-mode image and the optical image. Microbubble precursors were then placed at the bottom of the petri dish and both the optical and B-mode image were recorded as ultrasound was applied. Frequencies of 12.5, 15, 24, 30, 40, $50 \mathrm{MHz}$ were investigated. Optical images were recorded with a DSLR camera (T3i, Canon, Melville, NY) operating at 60 frames per second interlaced. 


\subsubsection{Stability of microbubble precursors}

Microbubble precursor stability was measured as a function of temperature. Microbubble precursors were fabricated, the pressure was released, but the particles were left in the capped syringe. The syringes were then placed at either 25 , or $37^{\circ} \mathrm{C}$ and the gas volume was recorded every 10 minutes. As the particles converted from the liquid phase back into the gas phase the gas volume would increase. Stability under agitation was monitored by placing particles on a rocker at $25^{\circ} \mathrm{C}$ and recording gas volume.

\subsubsection{Drug loading and triggered release}

The membrane-impermeable fluorophore calcein (Ex/Em 470/509, Sigma Aldrich St. Louis, MO) was used as a model drug. To produce calcein-loaded aaRBCs, $1 \mathrm{mM}$ calcein was added to the lysing solution. The aaRBCs were otherwise resealed and washed as above. Control calcein-loaded ghosts were produced in a similar manner except microbubbles or microbubble precursors were omitted from the lysing solution. To determine whether ultrasound could trigger drug release, samples of calcein-loaded aaRBCs and control calcein-loaded red blood cells ghosts were insonated using either the clinical ultrasound scanner or the small animal scanner. The samples were insonated for 10 minutes in color doppler mode at maximum power. The supernatant was sampled before and after insonation. The cells were then lysed with $0.1 \%$ Triton X (Sigma Aldrich St. Louis, MO) and the supernatant was sampled again to determine total loaded calcein. The absorbance of the supernatant samples was measured with a spectrophotometer (Nanodrop 1000, Thermo Scientific Wilmington, DE) at 499 nm and used to determine calcein concentration. Drug release was calculated as the difference between the absorbance before and after insonation, normalized to the total loaded calcein:

$$
\text { Release }=\frac{A b s_{\text {post }}-A b s_{\text {pre }}}{A b s_{\text {lyse }}-A b s_{\text {pre }}} \times 100 \% \text {. }
$$


Table 8.1: Boiling and melting point of selected common fluorocarbons

\begin{tabular}{ccc}
\hline Fluorocarbon & Boiling point $\left({ }^{\circ} \mathrm{C}\right)$ & Melting point $\left({ }^{\circ} \mathrm{C}\right)$ \\
\hline \hline Octafluoropropane $\left(\mathrm{C}_{3} \mathrm{~F}_{8}\right)$ & -37 & -183 \\
Decafluorobutane $\left(\mathrm{C}_{4} \mathrm{~F}_{10}\right)$ & -2 & -128 \\
Dodecafluoropropane $\left(\mathrm{C}_{5} \mathrm{~F}_{12}\right)$ & 28 & -115 \\
Perfluoro-n-hexane $\left(\mathrm{C}_{6} \mathrm{~F}_{14}\right)$ & 60 & -90 \\
Octafluorocyclobutane $\left(\mathrm{C}_{4} \mathrm{~F}_{8}\right)$ & -6 & -40 \\
\hline
\end{tabular}

\subsection{Results and Discussion}

\subsubsection{Microbubble precursor characterization}

DFB was used over higher boiling point fluorocarbons (Table 8.1) in order to minimize the amount of energy necessary to convert the liquid microbubble precursors back into a gas phase. Temperatures of at least $70^{\circ} \mathrm{C}$ have been reported when trying to convert liquid dodecafluoropentane $\left(\mathrm{C}_{5} \mathrm{~F}_{12}\right)$ nanodroplets back into gas despite a listed boiling point of $29^{\circ} \mathrm{C}[235]$. It has been suggested that the high Laplace pressures produced by the nanodroplets is responsible for the increased boiling points of liquid fluorocarbon nanodroplets [236]. Attempts to utilize octafluoropropane $\left(\mathrm{C}_{3} \mathrm{~F}_{8}\right)$ were unsuccessful as all the particles immediately converted back into the gas phase upon release of pressure.

Both sonication length and surfactant concentration were observed to affect microbubble precursor fabrication. If the particles were sonicated for less than 10 minutes, much of the liquid DFB would convert back into gas immediately upon release of pressure, and the droplets would skew towards larger $(\geq 1 \mu \mathrm{m})$ diameters. The presence or absence of albumin or tween, at concentrations up to $100 \mathrm{mg} / \mathrm{ml}$ and 10\%, respectively, did not appear to affect droplet diameter. Interestingly, liquid DFB sonicated in deionized water alone resulted in particles that survived after release of pressure and multiple centrifugations, suggesting the native fluorocarbon-water surface tension $(\approx 50 \mathrm{mN} / \mathrm{m})$ and low water solubility were sufficient to prevent liquid to gas conversion and particle fusion/ripening [39]. 
When using the fluorosurfactant Zonyl, surfactant concentration did affect droplet fabrication. Increasing Zonyl concentration was expected to decrease the diameter; however, increasing concentration instead decreased particle stability. Increasing Zonyl concentration resulted in increasing amounts of liquid immediately converting back into gas upon pressure release.

Initial sizing studies were performed with dynamic light scattering (DLS). When using DLS, no relationship was found between surfactant concentration or sonication times greater than 10 minutes and mean droplet diameter never went below 300 nm (Fig. 8.2a). A known limitation of DLS is its requirement for monodispersity. DLS does not measure individual particles, as in a Coulter counter, but instead attempts to fit a diameter that best explains the rate of signal decorrelation for a given viscosity. As a result, polydisperse populations are not accurately represented and a few large diameter particles can saturate the signal and hide smaller diameter particles. Using a different technology which individually tracks particles and calculates a diameter given the brownian motion and viscosity, a peak droplet diameter at $200 \mathrm{~nm}$ and a second smaller peak at $400 \mathrm{~nm}$ were measured (Fig. 8.2). The larger $400 \mathrm{~nm}$ diameter droplets and the wide distribution of droplet diameters likely masked the smaller diameters when measuring size with DLS. Furthermore, with nanoparticle tracking technology, a total particle concentration of $67 \times 10^{9}$ particles $/ \mathrm{mL}$ was estimated ${ }^{3}$. This estimated particle concentration represents a lower bound, however, as the technology only counts particles which have been tracked. For comparison, if all $6 \mathrm{~mL}$ of gaseous DFB is converted to liquid there would be enough volume to produce $9.7 \times 10^{12} 200 \mathrm{~nm}$ diameter particles. Despite the peaks at $200 \mathrm{~nm}$, all batches of microbubble precursors produced large diameter $(>1 \mu \mathrm{m})$ particles as measured on a Coulter counter and with optical microscopy.

The microbubble precursors were observed to be both stable and unstable. Upon first release of pressure after fabrication, some of the particles immediately converted back into gas.

\footnotetext{
${ }^{3}$ This represents a gas volume of $0.25 \mathrm{~mL}$. Assuming the measured distribution is representative of the population distribution, and all the gas in converted into droplets, the actual particle concentration may be 24 times larger
} 


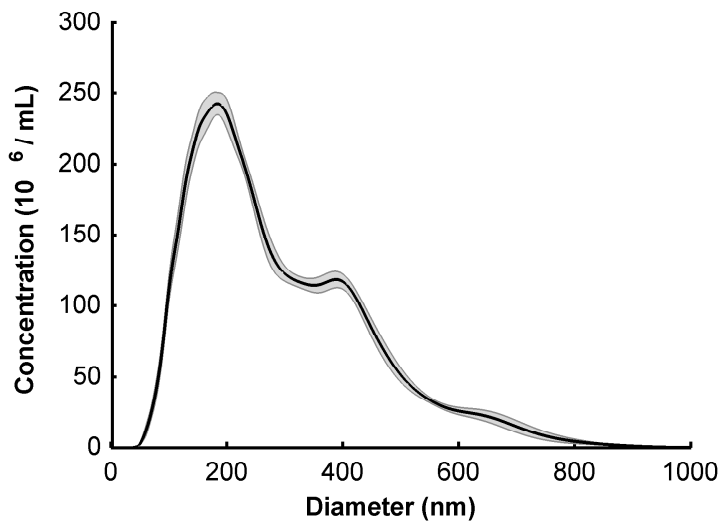

(a)

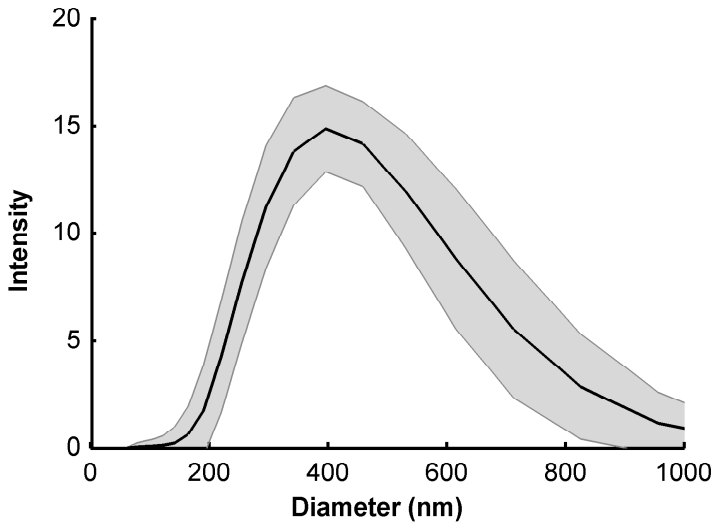

(b)

Figure 8.2: Diameter distribution of microbubble precursors via (a) nanoparticle tracking $(\mathrm{n}=5)$ and $(\mathrm{b})$ dynamic light scattering $(\mathrm{n}=10)$. Shaded bars represent standard deviation. By tracking individual particles, a peak at $200 \mathrm{~nm}$ is observed along with a secondary peak at $400 \mathrm{~nm}$. A lower bound of $67 \times 10^{9}$ particles $/ \mathrm{mL}$ was estimated.

Of the remaining particles, centrifugation at 16,000 RCF did not appear to affect diameter distribution (Fig. 8.3a). Incubation of the particles at dilute (1:100) concentrations overnight at $25^{\circ} \mathrm{C}$ resulted in no particle counts as measured by DLS. Microbubble precursors stored as a pellet were stable at $25^{\circ} \mathrm{C}$ for at least three months; however, sudden mechanical agitation of the pellet caused rapid particle conversion. Monitoring of evolved gas within a closed syringe demonstrated faster microbubble precursor conversion at $37^{\circ} \mathrm{C}$ and $25^{\circ} \mathrm{C}$ when under agitation as compared to when the particles were static at $25^{\circ} \mathrm{C}$ (Fig. 8.3b). Microbubble sizing of the remaining particles still resulted in a peak between 200-400 nm (Fig. 8.3b,c), suggesting there is a subset of small diameter particles that are ultra-stable. Attempts to visually observe particle conversion of large diameter precursors via microscopy on a $37^{\circ} \mathrm{C}$ heated stage were unsuccessful. Qualitatively, applying a steady negative pressure to the microbubble precursors $(50 \mathrm{kPa})$ for up to 10 minutes did not result in conversion of all the microbubble precursors as a pellet was still observed when the solution was centrifuged.

Insonation of the microbubble precursors with the high frequency ultrasound scanner was capable of causing rapid transition of the particles from liquid to gas (Fig. 8.4). When imaging in B-mode with the small animal scanner, transmit powers above $20 \%$ were capable 


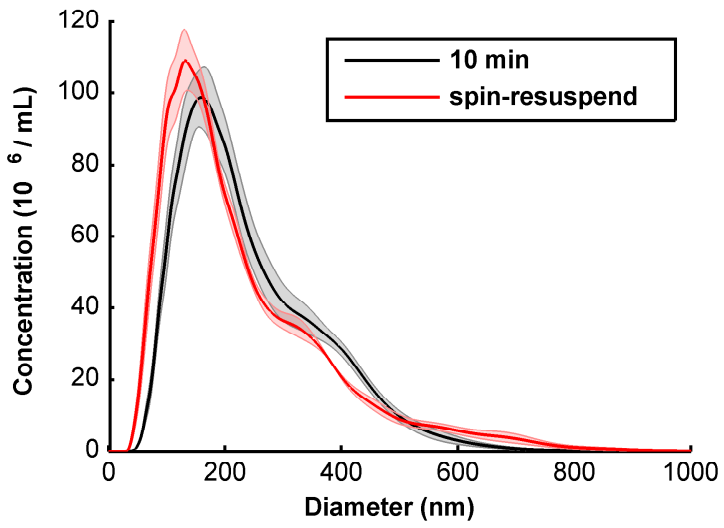

(a)

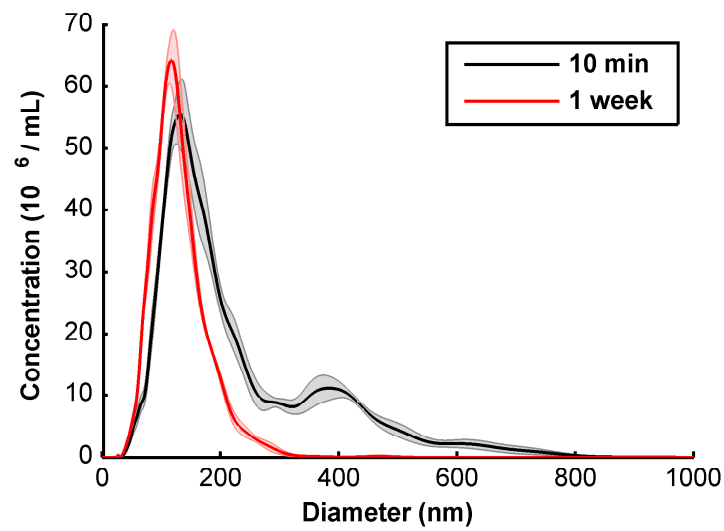

(c)

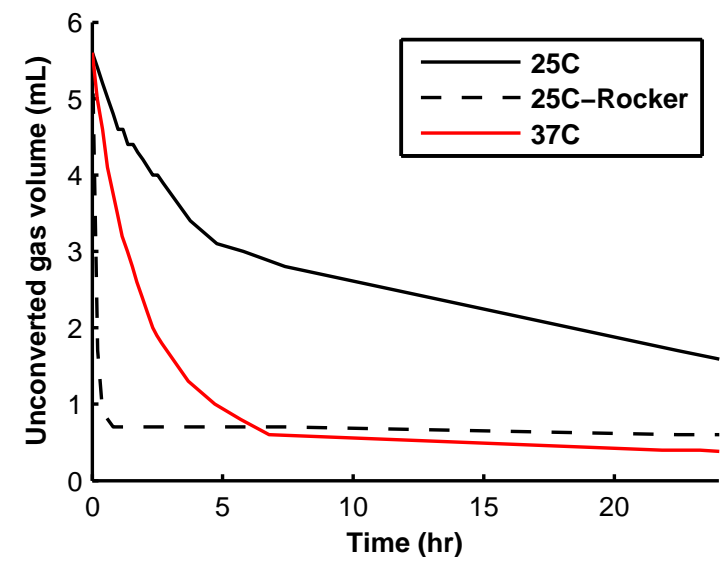

(b)

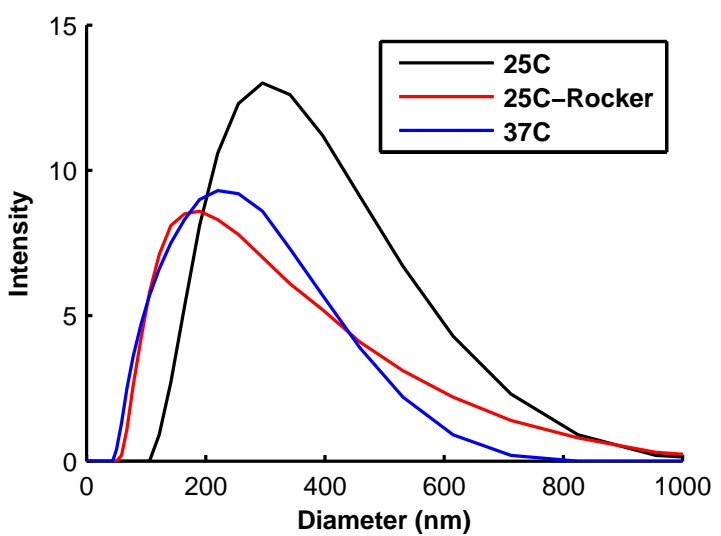

(d)

Figure 8.3: (a) Microbubble precursors were stable under centrifugation. (b) Incubation at $37^{\circ} \mathrm{C}$ and mechanical agitation caused the microbubble precursors to convert from liquid to gas at a faster rate when compared to particles remaining static at $25^{\circ} \mathrm{C}$. Following the stability study, (c) nanoparticle tracking and (d) dynamic light scattering of the samples measured a population of 200 - $400 \mathrm{~nm}$ diameter particles that remained even after the majority of the particles had converted back into gas.

of converting microbubble precursors at all frequencies (Table 8.2). A center frequency of 30 $\mathrm{MHz}$ required the lowest transmitted power to convert the microbubble precursors. When using doppler imaging, microbubble precursors were successfully converted at a transmit power of only $2 \%$, suggesting that the cycle length is important in acoustically converting the microbubble precursors. 


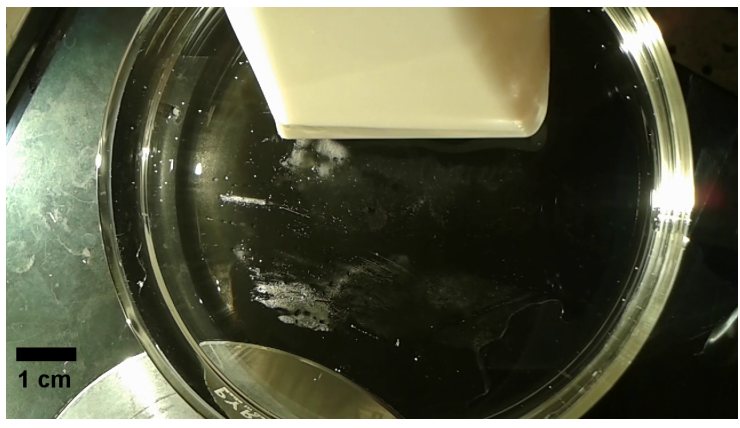

(a)

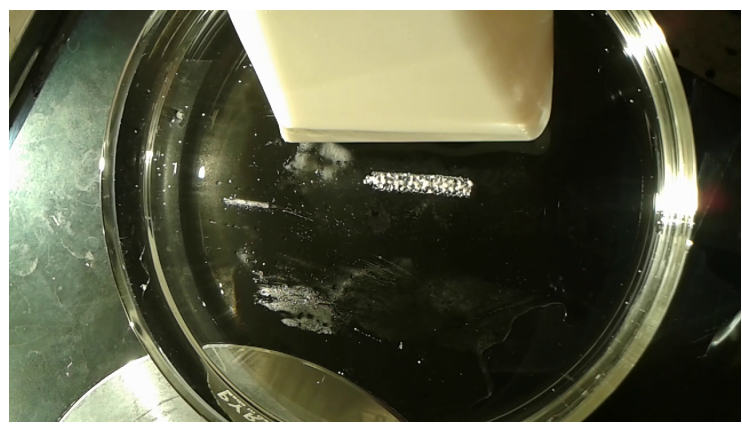

(b)

Figure 8.4: (a) Pre- (b) post- insonation of microbubble precursors with $12.5 \mathrm{MHz}, 100 \%$ transmit power, B-mode ultrasound.

Table 8.2: B-mode transmit power required to convert microbubble precursors into gas microbubbles

\begin{tabular}{ccc}
\hline Frequency $(\mathrm{MHz})$ & Transmitted power $(\%)$ & Transducer \\
\hline \hline 12.5 & 20 & MS200 \\
15 & 20 & \\
24 & 8 & MS400 \\
30 & 5 & \\
40 & 20 & MS700 \\
50 & 20 & \\
\hline
\end{tabular}

Vevo 2100 high frequency ultrasound scanner.

\subsubsection{Microbubble stability}

Although stable in storage, lipid-shelled decafluorobutane microbubble concentration linearly decreased with increasing centrifugation time at 1000, and $3000 \mathrm{RCF}$. At 10,000 RCF, microbubble loss was observed to be exponential with centrifugation time (Fig. 8.5).

\subsubsection{Red blood cell ghosts - Microbubble loading}

Fluorescence images overlaid on bright field images confirmed the loading of microbubbles inside the red blood cell ghosts. Four types of red blood cell ghosts were observed I) microbubble attached to the outer membrane of the red blood cell ghost; II) microbubble wholly contained within the red blood cell ghost cytoplasm III) microbubble attached to the inner leaflet of the microbubble ghost and 4) empty red blood cell ghost containing no microbubble (Fig. 8.6 a-d). Red blood cell ghosts with microbubbles wholly contained were 


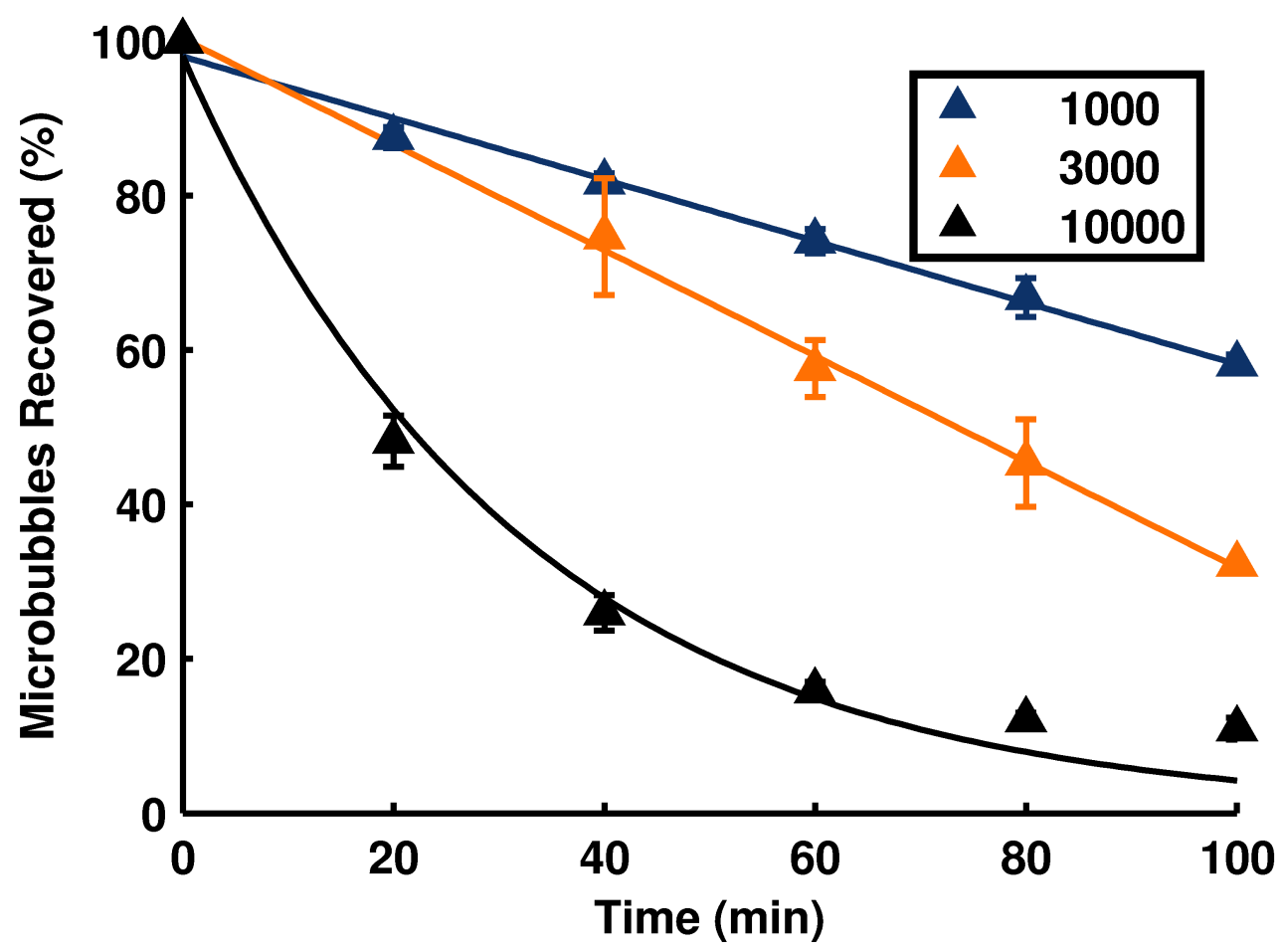

Figure 8.5: Microbubble loss at centrifugation speed of 1000, 3000, and 10,000 RCF $(\mathrm{n}=3)$. Microbubble loss at 1000 and 3000 RCF were linear, while microbubble loss at 10,000 RCF was best described by an exponential function. $\left(\mathrm{R}^{2}>0.98\right.$ for all fits).

confirmed by acquiring time lapse images and verifying microbubble motion within, but not outside, the red blood cell ghost.

Comparison of red blood cell ghosts to packed red blood cells demonstrates a loss of smaller diameters (Fig. 8.7. Optical observations suggest that that majority of the cells obtain a spherical shape after resealing rather than regaining their original biconcave discoid shape. Consequently, the size post-lysis is more likely a function of the salt concentration during resealing rather than the original size distribution of the packed red blood cells.

Using fluorescence-brightfield overlays to quantify loading, and counting types I, II, and III, (Fig. 8.6a-c), loading efficiency of aaRBCs was at most 20\%. The low efficiency is attributed to several factors. The diameter of the pores in the red blood cell membrane during lysis are on the order of $1 \mu \mathrm{m}$ [232, 228], while the mean microbubble diameter was $2 \mu \mathrm{m}$. Second, given their size and buoyancy, microbubbles are unlikely to be governed by 

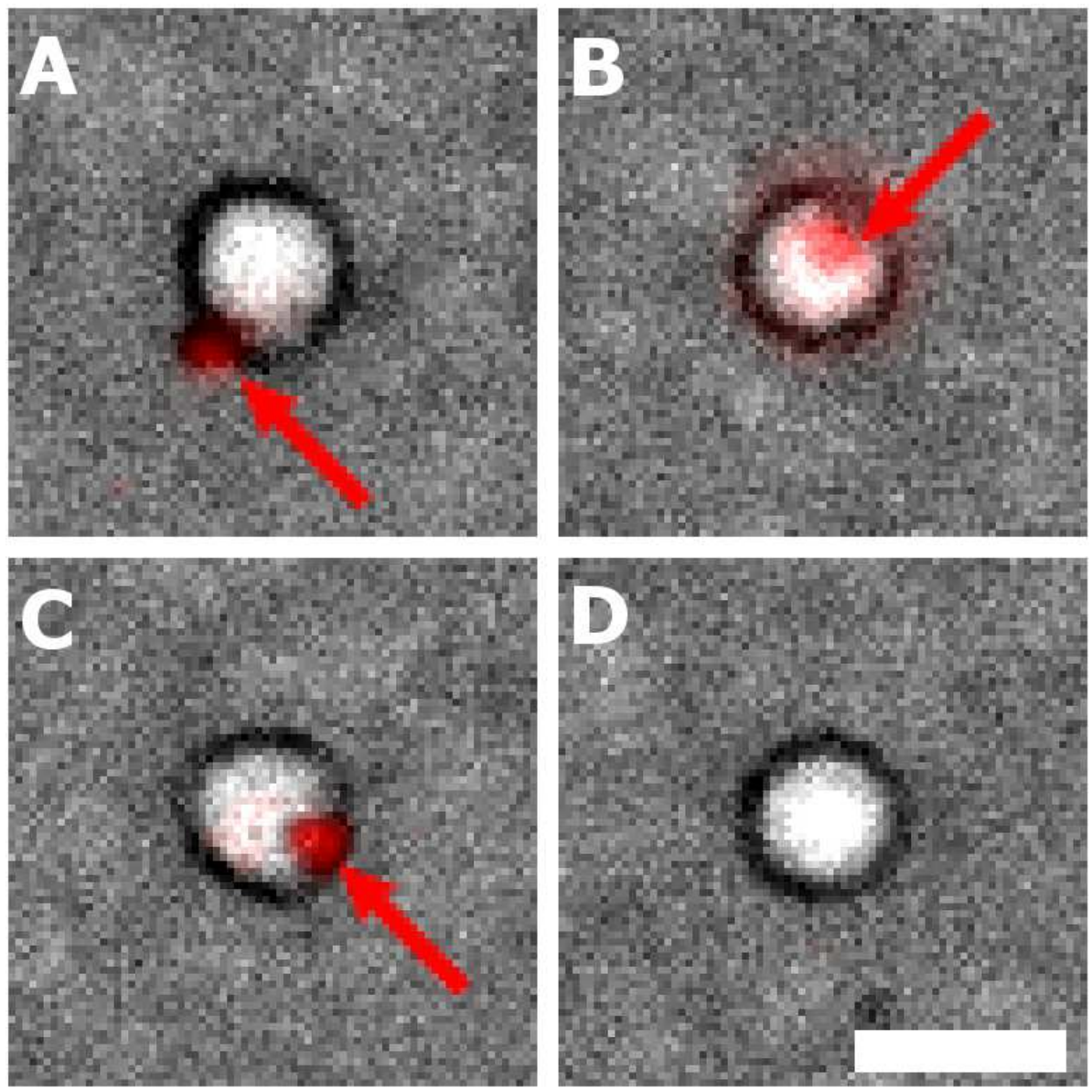

Figure 8.6: The four types of aaRBCs: (A) microbubble attached to the outer membrane of the red blood cell ghost; (B) microbubble wholly contained within the red blood cell ghost cytoplasm (C) microbubble attached to the inner leaflet of the microbubble ghost and (D) empty red blood cell ghost containing no microbubble. Arrows point to DiI-labeled microbubble. Scale bar $=10 \mu \mathrm{m}$.

the same diffusion kinectics as the small molecules that are more commonly loaded into red blood cells. Finally, microbubbles are typically not exposed to more than 100 RCF, as higher speeds cause increased microbubble dissolution and collapse [72]. To adequately wash the red blood cells ghosts and separate unencapsulated microbubbles, centrifugation speeds of at least 1000 RCF were required. Unfortunately, this may have caused microbubbles encapsulated within red blood cells to have collapsed (Fig. 8.5). 


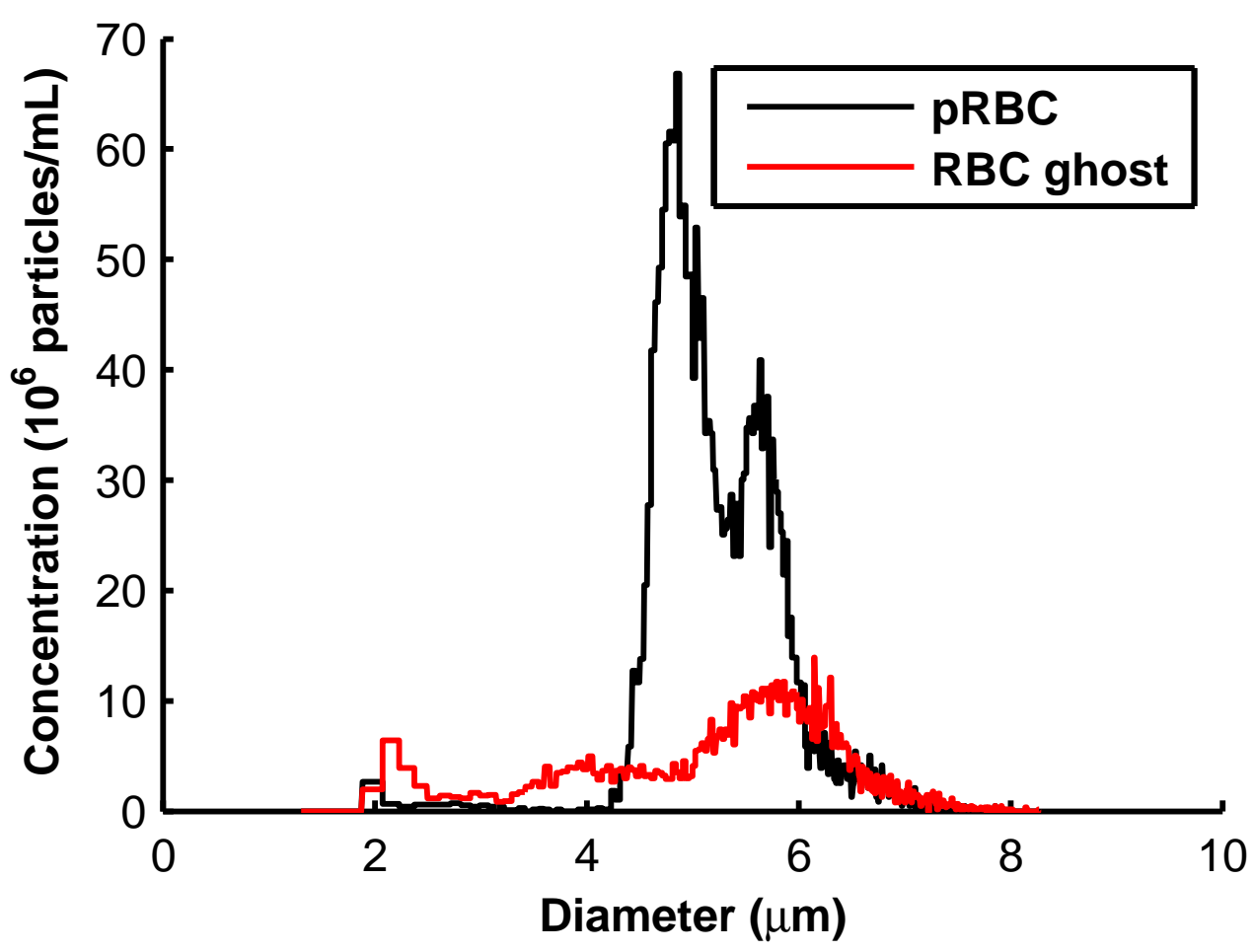

Figure 8.7: The diameter distribution of red blood cell shifts right following lysis and resealing.

\subsubsection{Red blood cell ghosts - Microbubble precursor loading}

Given the small size of the pores produced in the red blood cell membrane during hypotonic lysis, and the large loss of microbubbles during centrifugation, microbubble precursors were investigated rather than microbubbles. The microbubble precursors provide an approximately 5-fold decrease in diameter (i.e. a $200 \mathrm{~nm}$ diameter microbubble precursor should theoretically convert into a $1 \mu \mathrm{m}$ diameter microbubble [236]) and enhanced stability during centrifugation. As proof of concept, $200 \mathrm{~nm}$ diameter fluorescent polystyrene beads were loaded into red blood cells and loading was verified with fluorescent microscopy (Fig. 8.8).

Loading of red blood cells with microbubble precursors was attempted, but to date has been unsuccessful. One limitation of the microbubble precursors is the lack of a fluorescent label for optical verification of particle loading. A second limitation was the initial inability to reliably convert the microbubble precursors into microbubbles. Attempts to confirm loading by microscopy of the red blood cell ghosts on a heated stage in order to detect microbubble 


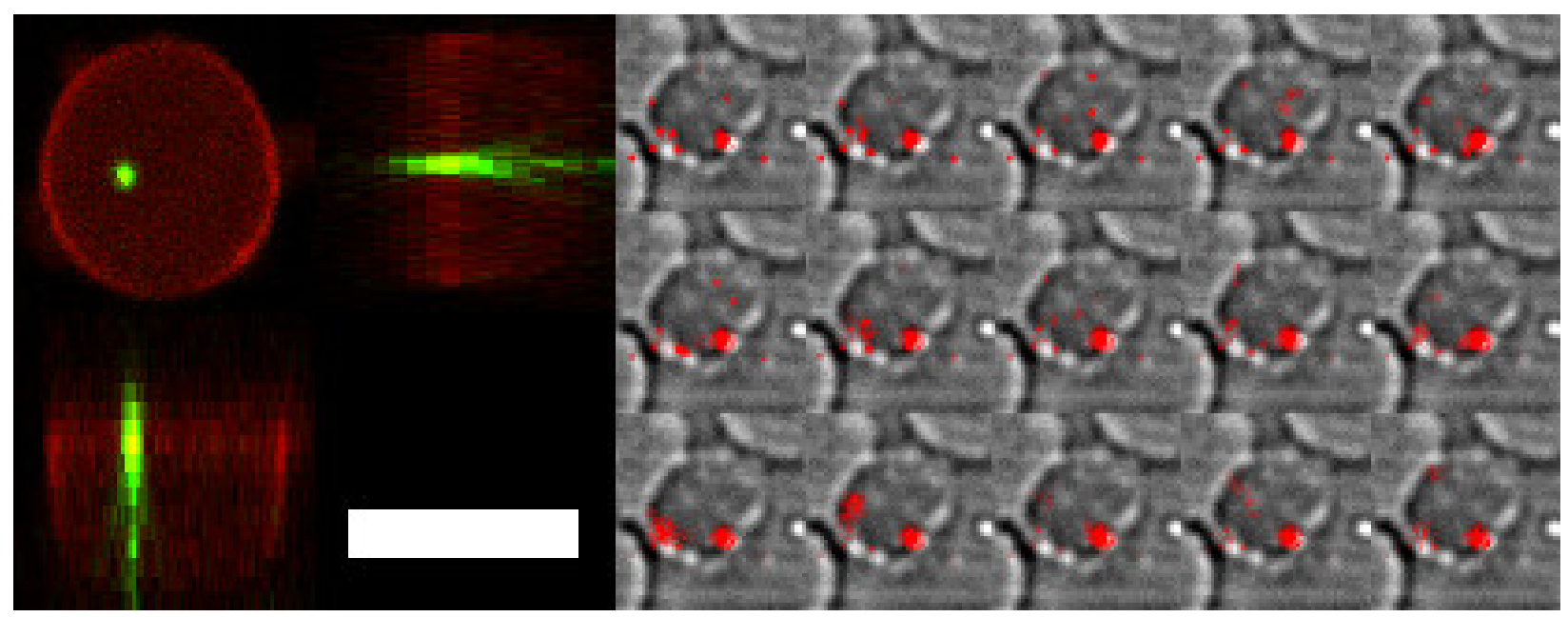

Figure 8.8: (Left) Confocal Z-stack of a polystyrene bead (green) in a red blood cell (red). (Right) Video montage of fluorescent overlay on a brightfield optical image showing successful loading of $200 \mathrm{~nm}$ diameter beads (red) into red blood cells (gray). Beads can be seen moving within, yet unable to escape, the cell. Scale bar $=5 \mu \mathrm{m}$ for image on left and $10 \mu \mathrm{m}$ for image on right.

precursor conversion into microbubbles was unsuccessful as the microbubble precursors did not reliably convert when exposed to heat.

\subsubsection{Triggered Release}

Validation of loading via triggered release was also equivocal. Insonation of calcein and microbubble loaded red blood cell ghosts resulted in a 14-fold increase in calcein release as compared to calcein-loaded red blood ghost controls (Fig. 8.9). A maximum of $20 \%$ of the total entrapped calcein was released from the calcein and microbubble loaded red blood cell ghosts. However, the calcein and microbubble loaded red blood cell ghosts contained type I, II, and III as well as residual unentrapped microbubbles. As a result, it was difficult to determine what percentage of calcein release came from red blood cell ghosts which had truly entrapped a microbubble, and what percentage of release was due to microbubbles abutting the red blood cell membrane.

Insonation of red blood cell ghosts loaded with microbubble precursors and calcein showed no significant difference compared to control. This may be a consequence of low microbubble precursor loading efficiency, failure of microbubble precursors to convert or failure of the 

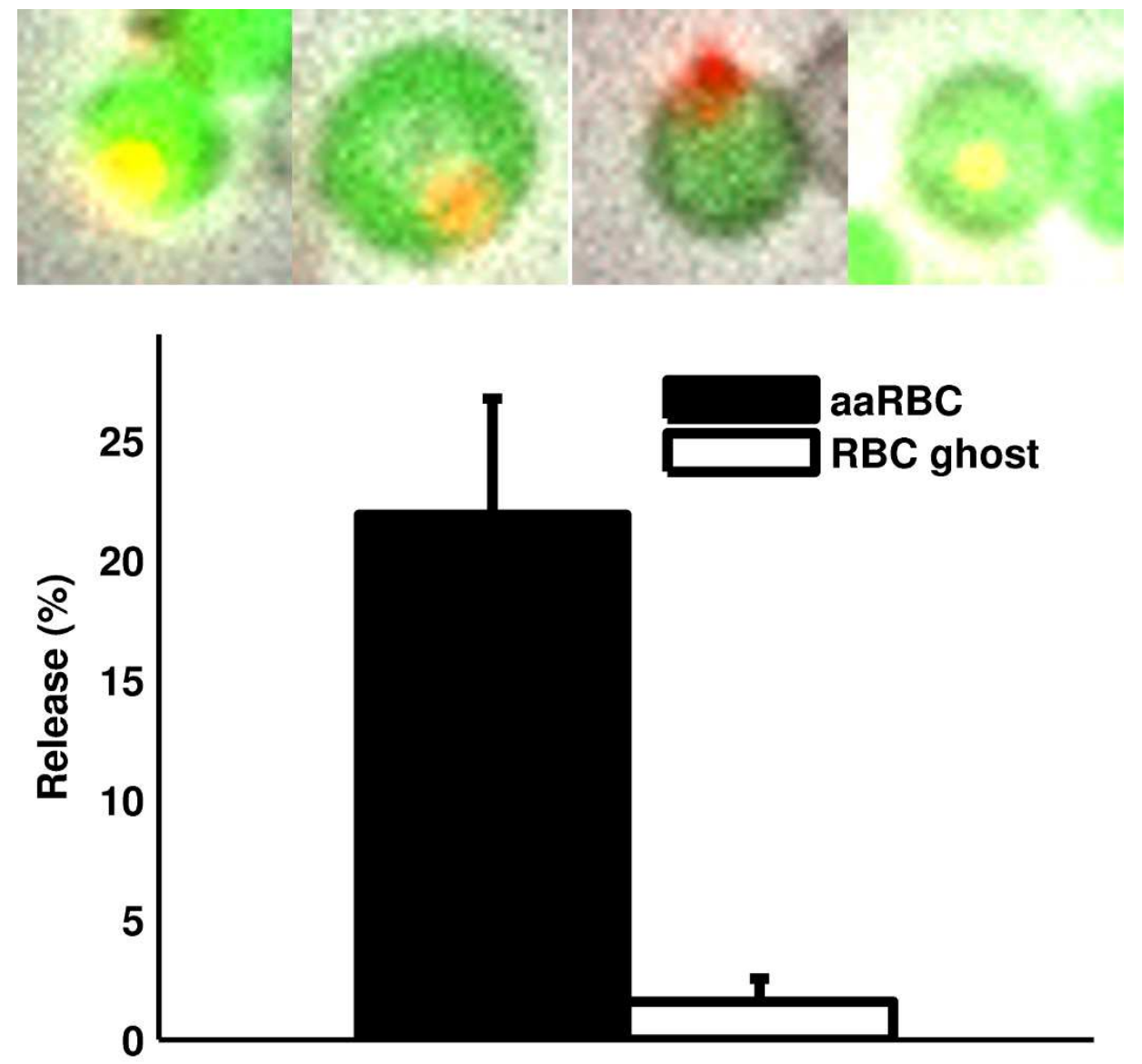

Figure 8.9: Top: Fluorescent images of calcein (green) and microbubbles (red) overlaid on bright field images of red blood cells showing successful production of calcein-loaded aaRBCs (image width $=10 \mu \mathrm{m})$. Bottom: Calcein-loaded aaRBCs showed a 14-fold increase in calcein release as compared to calcein-loaded red blood cell ghosts $(\mathrm{p}<0.001, \mathrm{n}=3)$.

converted microbubbles to significantly porate the red blood cells to allow calcein release. In the current insonation setup, a cloud of bubbles quickly developed within the eppendorf tube containing the sample. The source of these bubbles are likely unencapsulated microbubble precursors that were not washed away from the sample. The acoustic shadowing from this cloud may have prevented further conversion of microbubble precursors loaded within the red blood cell ghosts and thus limited calcein release. 


\subsection{Conclusion}

I hypothesized that loading a gas microbubble into a red blood cell ghost would provide an acoustic label to track therapy-loaded red blood cell ghost as well as provide an acoustic trigger for release of the entrapped therapeutic. Initial attempts to fabricate these particle using microbubbles were unsuccessful, likely a consequence of the similar size between the microbubble diameters and the pore in the red blood cell membrane during lysis. Proof or principle experiments utilizing $200 \mathrm{~nm}$ beads suggested that smaller nanodroplets could be loaded into red blood cell ghosts. However, initial attempts to load $200 \mathrm{~nm}$ diameter microbubble precursors composed of liquid decafluorobutane have also been unsuccessful. Increased concentrations of microbubble precursors, improved separation of unencapsulated microbubble precursors via differential centrifugation, and optimized insonation parameters to reliably convert the particles may enable successful production of acoustically active red blood cells. 


\section{Chapter 9}

\section{Conclusions and future directions}

\subsection{Final conclusions}

In this dissertation I have presented my work to enhance and extend the capabilities of gas microbubbles as ultrasound contrast agents. Part 1 of my dissertation involved development of microfluidics as a technology for microbubble production. Microfluidics is a unique technology that provides the ability to produce microbubbles in real-time via devices that have the potential to fit within the vasculature. Access to this technology is important because it provides a means to utilize larger diameter $(>10 \mu \mathrm{m})$ microbubbles while minimizing safety risks. As demonstrated by numerical models (Appendix B and section 6.3.3), larger diameter microbubbles should be better ultrasound contrast agents, as compared to smaller diameter microbubbles, especially when insonated at diagnostic ultrasound frequencies. Unfortunately, this enhancement has not been exploited as large diameters microbubbles may pose a safety risk due to the possibility of gas emboli formation. This is particularly true with current generation microbubbles that have been designed for longevity and are thus very stable. Dissolution models predict lipid-shelled fluorocarbon microbubbles to have lifetimes in the hours; as a result, a gas emboli formed by a stable microbubble has the potential to cause significant ischemia. Real-time production within the vasculature may be able to overcome 
this issue since microbubbles can be designed to be intentionally unstable. With access to transiently-stable microbubbles, large diameters can be investigated as dissolution can be relied upon to limit gas emboli formation. Hence, microfluidics were investigated to achieve real-time production via a method than can be miniaturized.

In Chapter 2 I presented work on a novel interconnect design to reduce the footprint of microfluidic devices by placing the microfluidic device within a pressurized liquid chamber. This design eliminates the need for bulky interconnects, thus enabling a reduction in the overall device dimensions. In this chapter, I also provide initial in vitro evidence that a microfluidic device can produce a sufficient number of microbubbles in real-time to achieve enhanced acoustic contrast at both diagnostic frequencies $(7 \mathrm{MHz})$ and intravascular ultrasound (IVUS) frequencies $(45 \mathrm{MHz})$. The ability to image at IVUS frequencies is significant because ultimately we envision pairing an IVUS transducer and a microfluidic device within a single catheter (Fig. 9.1) for real-time imaging and drug delivery within the vasculature.

In Chapter 3, I presented work on the development of an analytical model to predict the microbubble production rate as a function of liquid flow rate and gas pressure. In addition to providing access to transiently-stable microbubbles, microfluidics enables selection of the microbubble diameter and production rate through adjustment of the gas and liquid input parameters. Models such as this will be important if microfluidic devices are to be used in situ and microbubble concentration are to be adjusted in real-time. Real-time adjustment would enable an operator to achieve concentrations optimized for the clinical situation. For example, low concentrations to reduce acoustic shadowing in imaging applications and high concentrations to enhance sonoporation in therapeutic applications. Current methods for measuring microbubble diameter and concentration require either a high speed camera or a Coulter counter, neither of which are compatible with vascular-sized microfluidic devices and thus would require the microfluidic device to be removed from the body for microbubble characterization. With a model, microbubble concentration could be predicted without having to remove the microfluidic device from the vasculature. 


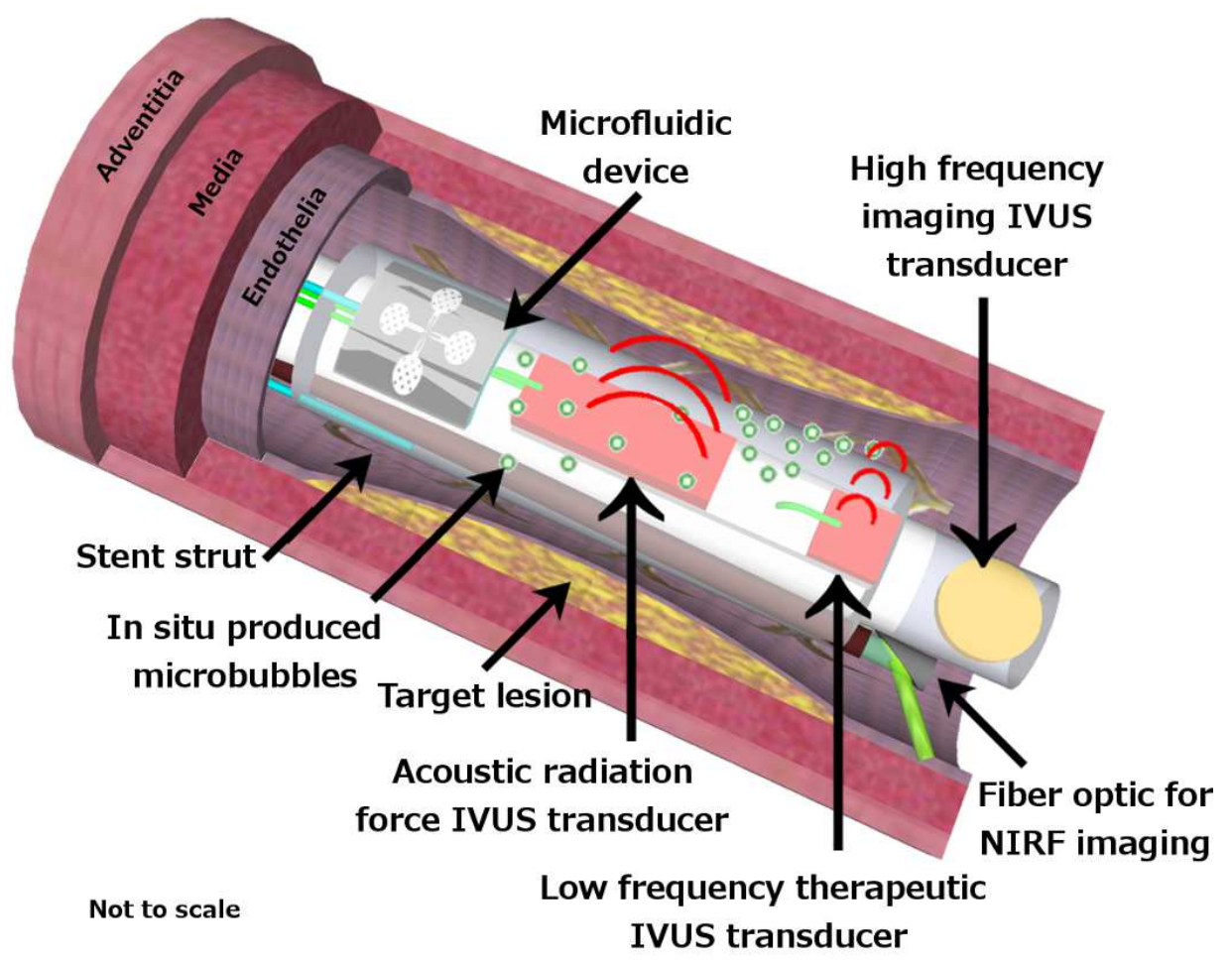

Figure 9.1: Schematic of the proposed multi-function IVUS catheter containing a microfluidic device for in situ microbubble production and IVUS transducers for microbubble pushing, therapy, and imaging.

In Chapter 4, I presented work on the development of albumin as a biocompatible shell that is optimized for the production of transiently-stable microbubbles via a microfluidic device. Next generation microbubbles typically seek long-term stability for the microbubble, and thus use a variety of lipids, protein, and polymer shells and low solubility gases. For in situ microbubble production, the goal is to produce transiently-stable microbubbles: that is stable enough to produce acoustic contrast, yet not too stable to increase the risk of gas emboli formation. Furthermore, since the microbubbles are to be produced in situ, all components of the shell material must be biocompatible since excess material will be released directly into the bloodstream alongside the microbubbles. To that end, due to their biocompatibility, albumin and dextrose were investigated as stabilizing agents for nitrogen gas microbubbles. These albumin-stabilized microbubbles were measured to have a half-life under $10 \mathrm{~s}$ and, again, were shown to be sufficient in providing acoustic contrast. Significantly, we also demonstrated the production of microbubbles directly from plasma. The ability to 
stabilize microbubbles with a patient's own plasma could reduce the requirement for storage of shell components, which could be significant in developing countries that have limited access to refrigeration, as well as minimize any immunological reaction that may occur with foreign shell proteins.

In Chapter 5, I presented work investigating the potential of in situ produced microbubbles to effect drug delivery in conjunction with ultrasound. To more accurately simulate the vasculature, cells were placed in an in vitro flow chamber. Microbubbles were then produced in situ via a microfluidic device placed directly upstream of the cells. Similar to the environment in vivo, microbubbles continuously flowed past the cells rather than being in static contact over the duration of the insonation. Using in situ produced microbubbles, we achieved delivery up to $80 \%$ of the cells at pressures as low as $100 \mathrm{kPa}$. Similar to contrast applications, in situ produced microbubbles for therapy provides a new paradigm for achieving ultrasound potentiated therapies such as sonoporation of sonothrombolysis. With access to large diameter microbubbles, pore formation in cells could be enhanced or the rate of clot dissolution could be increased.

Finally, in Chapter 6, I presented the first report of direct administration of microfluidicproduced microbubbles into the vasculature for acoustic contrast. Large diameter $(10-20 \mu)$ albumin-stabilized microbubbles were administered from the microfluidic device output directly into venous circulation via a tail-vein catheter. Imaging at $12.5 \mathrm{MHz}$ demonstrated contrast in the right ventricle and nearly complete clearance after one pass through the lungs. Most significantly, no respiratory or cardiac distress was observed in any of the animals and all animals survived the procedure. Overall, Part 1 demonstrated the rationally-guided development of a microfluidic device that can produce transiently-stable microbubbles and has the potential to be miniaturized, ultimately culminating in in vivo verification that transiently-stability can abrogate the risks of large diameter microbubbles.

Part 2 of this dissertation explores additional ways to enhance the capabilities of microbubbles. In Chapter 7, I developed a numerical framework for designing transducers optimized 
for producing radiation force. Although this specific study investigated radiation force for acoustic radiation force impulse (ARFI) imaging, the same transducer could be used for the application of radiation force to in situ produced microbubbles. Given the short lifetimes of transiently-stable microbubbles, radiation force may be important for concentrating the microbubbles at the vessel wall in order to enhance imaging and drug delivery. Finally, in Chapter 8 I reported on initial attempts to combine the benefits of red blood cell ghosts and microbubbles to produce a new drug delivery vehicle with a large payload and an acoustic trigger. Although initial results were negative, microbubble precursors may provide a means to efficiently produce this novel drug delivery vehicle.

\subsection{Limitations}

As with all research, there are limitations to the work presented here. For the flooded microfluidic design, the initial goal was to place the microfluidic device inside the pressurized chamber. Unfortunately, current polymer based microfluidic devices appear unable to withstand the pressures necessary to achieve microbubble production. As a result, the devices would collapse, stopping microbubble production. Development of glass-based, or other stiff material, microfluidic devices may remedy this issue. For the microfluidic model, validation across multiple devices, and different shell and gas parameters, is necessary to produce a truly representative model. Furthermore, improved, industrial-grade, microfabrication techniques will likely be necessary to reduce device-to-device variations that may affect microbubble production and thus the model. Regarding monodispersity, although the albumin stabilized microbubbles remained monodisperse within the microfluidic device, it is unlikely, as a population, that the microbubbles stay monodisperse. This could make it difficult to optimize the acoustic frequency to the resonant frequency of the microbubble. Finally, for drug delivery, only a single acoustic frequency was tested. Additional frequencies are necessary to generalize the results. 


\subsection{Future directions}

Most importantly, though, this work lends itself to numerous next steps. The next logical step, which is currently underway, is the fabrication of a microfluidic device capable of fitting within the vasculature. While this will likely require a redesign of the original flow-focusing design used in the previous studies, the advantages of the flooded design should become more evident. Aside from development of the microfluidic device, a rigorous comparison, both in vitro and in vivo, of the acoustic and therapeutic properties between large diameter transiently-stable microbubble and current generation small diameter stable microbubbles needs to be conducted cross appropriate clinical frequencies and pressures to verify the benefits predicted by the acoustic models. Finally, microfluidic devices should be put through a battery of failure tests to better understand when and how the devices fail in order to design appropriate safety mechanisms.

Similarly, while finite element models have previously been validated for simulating ARFI imaging phenomena, there is no substitute for experimental results. To that end, the optimized ARFI imaging transducer should be fabricated and its imaging characteristics compared to a conventional linear array. Once fabricated, additional experiments measuring the transducers abilities for directing microbubbles via radiation force could be conducted.

Regarding the development of acoustically active red blood cells, several paths could be taken. With both microbubble and microbubble precursor loading, improved sorting methods will be needed to improve loading efficiency. While the absolute number of red blood cell ghosts may be large, given the current loading method, it is unlikely that loaded red blood cell ghosts will be a large percentage of the total ghosts. Magnetic or other non-centrifugation methods may enable separation of microbubble-loaded red blood cell ghosts. Tight control over climate and mechanical vibration may improve the success of differential centrifugation for separating microbubble-precursor loaded red blood cell ghosts. Initial characterization of the microbubble precursors has shown that doppler ultrasound is the most consistent method for converting the precursors into gas microbubbles. Consequently, once sorted it 
may by possible to observe precursor conversion within a red blood cell ghost and confirm particle loading by combining optical imaging with doppler ultrasound. Once loading has been confirmed, acoustic imaging in blood needs to be performed to verify that the loaded red blood cell ghosts can be tracked. In addition, the acoustic parameters that enable imaging versus drug release need to be determined. Finally, the acoustically active red blood cells will need to be administered in vivo to confirm circulation time and the potential for site specific targeting. 


\section{Appendices}




\section{Appendix A}

\section{Publications}

1. L. C. Phillips, A. H. Dhanaliwala, A. L. Klibanov, J. A. Hossack, and B. R. Wamhoff, "Focused ultrasound-mediated drug delivery from microbubbles reduces drug dose necessary for therapeutic effect on neointima formation-brief report," Arterioscler. Thromb. Vasc. Biol., vol. 31, no. 12, pp. 2853-2855, Dec. 2011.

2. A. H. Dhanaliwala, J. A. Hossack, and F. W. Mauldin, "Assessing and improving acoustic radiation force image quality using a 1.5-D transducer design," IEEE Trans Ultrason Ferroelectr Freq Control, vol. 59, no. 7, pp. 1602-1608, Jul. 2012.

3. F. W. Mauldin Jr, A. H. Dhanaliwala, A. V. Patil, and J. A. Hossack, "Real-time targeted molecular imaging using singular value spectra properties to isolate the adherent microbubble signal," Phys Med Biol, vol. 57, no. 16, pp. 5275-5293, Aug. 2012.

4. A. H. Dhanaliwala, J. L. Chen, S. Wang, and J. A. Hossack, "Liquid flooded flow-focusing microfluidic device for in situ generation of monodisperse microbubbles," Microfluid Nanofluidics, vol. 14, no. 3-4, pp. 457-467, Mar. 2013.

5. A. J. Dixon, A. H. Dhanaliwala, J. L. Chen, and J. A. Hossack, "Enhanced Intracellular Delivery of a Model Drug Using Microbubbles Produced by a Microfluidic Device," Ultrasound Med Biol, vol. 39, no. 7, pp. 1267-1276, Jul. 2013. 
6. S. Wang, A. H. Dhanaliwala, J. L. Chen, and J. A. Hossack, "Production rate and diameter analysis of spherical monodisperse microbubbles from two-dimensional, expanding-nozzle flow-focusing microfluidic devices," Biomicrofluidics, vol. 7, no. 1, pp. 014103-014103-12, Jan. 2013.

7. J. L. Chen, A. H. Dhanaliwala, A. J. Dixon, A. L. Klibanov, and J. A. Hossack, "Synthesis and Characterization of Transiently Stable Albumin-Coated Microbubbles via a Flow-Focusing Microfluidic Device," Ultrasound Med Biol, vol. 40, no. 2, pp. 400-409, Feb. 2014.

8. A. H. Dhanaliwala, J. Chen, J. P. Kilroy, L. C. Phillips, A. J. Dixon, B. R. Wamhoff, A. L. Klibanov, and J. A. Hossack, "Intravascular Ultrasound-Based Imaging and Drug Delivery," IEEE Trans Ultrason Ferroelectr Freq Control, vol. submitted, 2014.

9. A. H. Dhanaliwala, A. J. Dixon, D. Lin, J. L. Chen, A. L. Klibanov, and J. A. Hossack, "In vivo imaging of microfluidic-produced microbubbles," Biomedical microdevices, vol. submitted, 2014.

10. J. P. Kilroy, A. H. Dhanaliwala, A. L. Klibanov, D. K. Bowles, B. R. Wamhoff, and J. A. Hossack, "Reducing Neointima Formation in a Swine Model with IVUS and Rapamycin Microbubbles," Nature Medicine, no. submitted, 2014. 


\section{Appendix B}

\section{Numerical evaluation of the scattering cross section of a microbubble}

\section{B.1 Theory}

For a free gas microbubble in water, the scattering cross section can be calculated from [135]:

$$
\sigma_{X C}=\frac{4 \pi R^{2}}{\left[\left(\frac{f_{R}}{f_{c}}\right)^{2}-1\right]^{2}+\delta_{\text {damp }}^{2}}
$$

where $\sigma_{X C}$ is the scattering cross section, $R$ is the microbubble radius, $f_{R}$ is the resonance frequency of the microbubble, $f_{c}$ is frequency of the insonation wave, and $\delta_{\text {damp }}$ is the damping constant. The resonance frequency of a gas bubble in water can be calculated from [135]:

$$
f_{R}=\frac{1}{2 \pi R} \sqrt{\frac{3 \gamma p_{a m b}}{\rho_{L}}}
$$

where $\gamma$ is the ratio of specific heats for the gas, $p_{a m b}$ is the ambient pressure, and $\rho_{L}$ is the density of water. The damping constant can be broken into its constitutive components, 
which for a free, unshelled, gas bubble are [135]:

$$
\delta_{\text {damp }}=\delta_{\text {radiation }}+\delta_{\text {viscosity }}+\delta_{\text {thermal }}
$$

In eqn. B.3, $\delta_{\text {radiation }}$ is the energy lost due to re-radiation of the acoustic wave (i.e. the scattered wave):

$$
\delta_{\text {radiation }}=\frac{2 \pi f_{c} R}{c},
$$

where $c$ is the speed of sound in water, and $\delta_{\text {viscosity }}$ is the energy lost due to the viscous forces at the gas-liquid interface:

$$
\delta_{\text {viscosity }}=\frac{4 \eta_{L}}{\rho_{L} 2 \pi f_{c} R^{2}}
$$

where $\eta_{L}$ is the viscosity of water. $\delta_{\text {thermal }}$ is the energy lost due to heat transfer between the gas, as it heats up during compression, and the surrounding liquid. The $\delta_{\text {thermal }}$ term can be eliminated by using an effective viscosity, often estimated as $2 \eta_{L}$ [170].

\section{B.2 Results and Discussion}

When the product of microbubble radius and frequency are small, $f_{c} R<<2.4$, scattering cross section increases to the $6^{\text {th }}$ power of radius and $4^{\text {th }}$ of frequency until resonance is reached. At resonance scattering cross section is maximal after which cross section decreases. At $f_{c} R>5$, scattering cross section becomes independent of frequency and increases with the square of the radius (Fig. B.1c).

Insonation frequency and microbubble radius combinations that maximize the scattering cross section should maximize the reflected acoustic wave, resulting in improved contrast enhancement. Consequently, the utilization of large microbubbles ( $>10 \mu \mathrm{m}$ diameter $)$, aside 


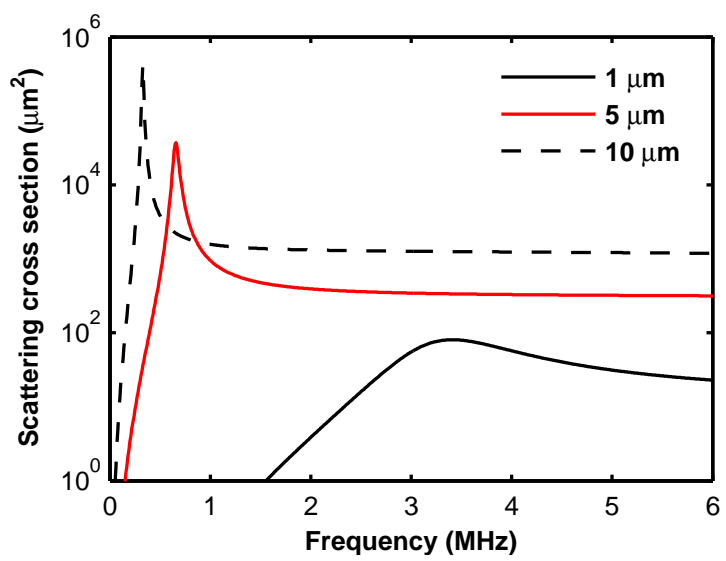

(a)

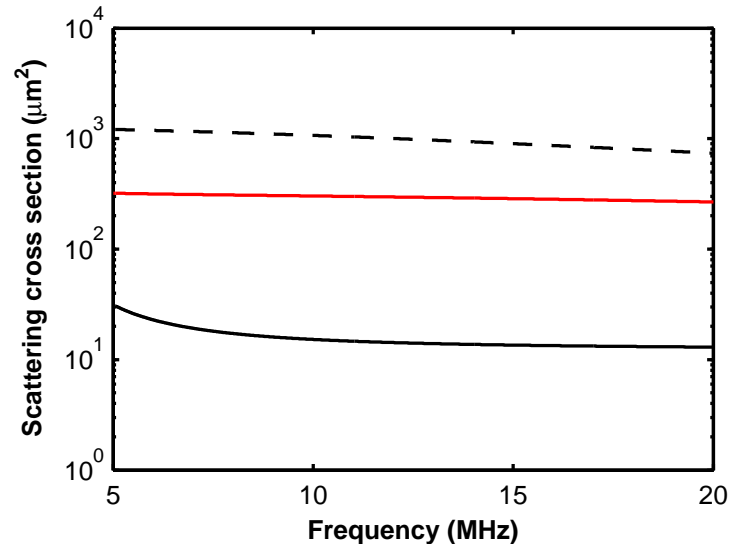

(b)

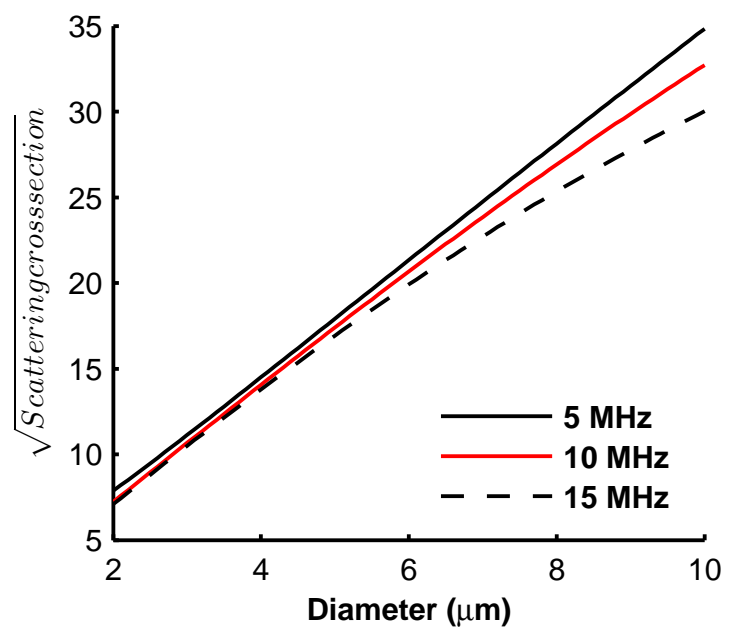

(c)

Figure B.1: (a) The scattering cross section of microbubbles increases with frequency until a maximum is achieved at the resonance frequency of the microbubble. (b) Following resonance the cross section becomes independent of frequency. Even with a resonance peak, over clinically relevant imaging frequencies the scattering cross section of larger microbubbles is larger than the scattering cross section of smaller microbubbles.

from any emboli risk, should produce an improvement in image contrast as compared to smaller microbubbles (Fig. B.1a,b). It should be noted, however, that the above equations for scattering cross section assume a single microbubble. As microbubble concentration increases, attenuation and multi-path effects will influence the actual amount of reflected acoustic energy, and thus image contrast [237, 29]. 


\section{Appendix C}

\section{Fluid dynamic relationships}

Reynolds number:

$$
R e=\frac{\rho_{L} u l}{\mu},
$$

where $\rho_{L}$ is the fluid density, $\mu$ is the dynamic viscosity, $u$ is mean flow velocity, and $l$ is the characteristic length.

Weber number:

$$
W e=\frac{\rho_{L} u^{2} l}{\sigma},
$$

where $\sigma$ is the surface tension.

Capillary number:

$$
C a=\frac{\mu u}{\sigma},
$$

Hydraulic diameter of a rectangular cross section tube $\left(D_{h}\right)$ :

$$
D_{h}=\frac{2 h w}{h+w},
$$


where $h$ and $w$ are the height and width, respectively, of the channel at the nozzle [115].

Shear stess in laminar flow:

$$
\tau_{s}=\frac{6 \eta_{L} Q_{l}}{w h^{2}},
$$

where $\eta_{L}$ is the fluid viscosity, $Q_{l}$ is the volumetric flow rate, $w$ is the width of chamber, and $h$ is the height of chamber [159]. 


\section{Appendix D}

\section{Dissolution model of}

\section{fluorocarbon-nitrogen microbubbles}

\section{D.1 Theory}

While the original Epstein-Plesset model described in section 6.3.4 is suitable for simulating dissolution of bubbles filled air, the model only accounts for a single gas. As a result, the Epstein-Plesset model is unsuitable for simulating dissolution of microbubbles composed of low solubility gases such as fluorocarbons since the bubble quickly becomes a mixture of both fluorocarbon and air. To account for the influx of dissolved air from the surrounding media into the microbubble in conjunction with the efflux of fluorocarbon out of the microbubble, Kabalnov et al. re-derived the relationship between microbubble radius and gas flux [103]:

$$
\begin{aligned}
& \frac{d F}{d \tau}=-\frac{3 L_{F} F}{\rho^{2}} \\
& \frac{d N}{d \tau}=-\frac{3 \delta L_{N}}{\rho^{2}}\left(A-\rho^{3}\right) \\
& \frac{d \rho}{d \tau}=\frac{\frac{d F}{d \tau}+\frac{d N}{d \tau}}{2 \mu \rho+3(1+\nu) \rho^{2}}
\end{aligned}
$$


Table D.1: Fluorocarbon microbubble dissolution parameters

\begin{tabular}{ccc}
\hline Symbol & Parameter & Value \\
\hline \hline$R_{0}$ & Initial radius & \\
$D_{F}$ & Octafluoropropane diffusion constant & $0.715\left(25^{\circ} \mathrm{C}\right) \times 10^{-9} \mathrm{~m}^{2} \mathrm{~s}^{-1}[238,103]$ \\
$D_{N}$ & Nitrogen diffusion constant & $2.01\left(25^{\circ} \mathrm{C}\right), 2.83\left(40^{\circ} \mathrm{C}\right) \times 10^{-9} \mathrm{~m}^{2} \mathrm{~s}^{-1}[175]$ \\
$L_{F}$ & Octafluoropropane Ostwald coefficient & $*$ \\
$L_{N}$ & Nitrogen Ostwald coefficient & $15880\left(25^{\circ} \mathrm{C}\right), 14000\left(40^{\circ} \mathrm{C}\right) \times 10^{-6}[176]$ \\
$p_{\text {blood }}$ & Circulation overpressure & $4300 \mathrm{~Pa}$ \\
$p_{\text {amb }}$ & Ambient pressure & $101.3 \mathrm{kPa}$ \\
$\sigma$ & Surface tension & $0.05 \mathrm{~N} \mathrm{~m}^{-1}[178]$ \\
\hline
\end{tabular}

*The values for the Ostwald coefficient of octafluoropropane are significantly different if extrapolated from the values in [103] or calculated from solubility and vapor pressure, as explained below.

The equations have been nondimensionlized such that:

$$
\mu=\frac{2 \sigma}{p_{a m b} R_{0}} ; \nu=\frac{p_{\text {blood }}}{p_{a m b}} ; \rho=\frac{R}{R_{0}} ; \delta=\frac{D_{N}}{D_{F}} ; \tau=\frac{D_{f} t}{R_{0}^{2}} ; p_{N}=\frac{N p_{a m b}}{\rho^{3}} ; p_{F}=\frac{F p_{a m b}}{\rho^{3}} ;
$$

where, $R_{0}$ is the initial radius, $D_{F}$ is the diffusion coefficient of the gaseous fluorocarbon in water, $D_{N}$ is the diffusion coefficient of gaseous nitrogen in water, $L_{F}$ is the fluorocarbon Ostwald coefficient ${ }^{1}, L_{N}$ is the nitrogen Ostwald coefficient, $p_{\text {blood }}$ is the extra pressure due to systemic blood pressure, $p_{a m b}$ is the ambient pressure, and $\sigma$ is surface tension. Parameter definitions and values can be found in Table D.1.

These equations can be numerically solved by treating them as a coupled set of equations with the following initial conditions:

$$
\begin{aligned}
& \rho(0)=1 \\
& F(0)=(\mu+\nu+1) X_{F} \\
& A(0)=(\mu+\nu+1)\left(1-X_{F}\right)
\end{aligned}
$$

\footnotetext{
${ }^{1}$ The Ostwald coefficient is the ratio of the water solubility of the gas to the saturated vapor pressure of the gas. If using ideal gases, the Ostwald coefficient can be computed from the Henry's constant for the gas saturation in water.
} 
Table D.2: Octafluoropropane coefficients

\begin{tabular}{ccc}
\hline Symbol & Parameter & Value \\
\hline \hline$V_{m}$ & Molar volume & $142.14 \mathrm{~cm}^{3} \mathrm{~mol}^{-1}\left(25^{\circ} \mathrm{C}\right)[238]$ \\
$D_{F}$ & Octafluoropropane diffusion constant & $0.715\left(25^{\circ} \mathrm{C}\right) \times 10^{-9} \mathrm{~m}^{2} \mathrm{~s}^{-1}[239,103]$ \\
& Octafluoropropane vapor pressure & $0.868\left(25^{\circ} \mathrm{C}\right), 1.275\left(40^{\circ} \mathrm{C}\right) \mathrm{MPa}[12]$ \\
& Octafluoropropane water solubility & \\
& Fitted & $0.19 \mathrm{~mol} \mathrm{~m}^{-3}[240,103]$ \\
& Measured & $0.03 \mathrm{~mol} \mathrm{~m}{ }^{-3}($ personal communication $)$ \\
$L_{F} \quad$ Octafluoropropane Ostwald coefficient & $548\left(25^{\circ} \mathrm{C}\right), 392\left(40^{\circ} \mathrm{C}\right) \times 10^{-6}$ \\
& Fitted & $85.7\left(25^{\circ} \mathrm{C}\right), 61.3\left(40^{\circ} \mathrm{C}\right) \times 10^{-6}$ \\
\hline
\end{tabular}

Saturated vapor pressure was converted to saturated vapor concentration by dividing by the universal gas constant $(R)$ and temperature.

where $X_{F}$ is the initial amount of fluorocarbon in the microbubble $(1=100 \%, 0=0 \%)$.

A major discrepancy, however, occurs when selecting the diffusion and Ostwald coefficient for octafluoropropane. First, the diffusion coefficient of octafluoropropane is unknown and is calculated from its molar volume $-142.14 \mathrm{~cm}^{3} \mathrm{~mol}^{-1}$ [238] - via the Hayduk-Laudie relationship $[239,103]^{2}$ :

$$
D=13.26 \times 10^{-5} V_{m}^{-0.589}
$$

where $D$ is the diffusion coefficient and $V_{m}$ is the molar volume. This relationship does not correct for temperature, however, and so is likely an underestimate of the actual fluorocarbon dissolution at $37^{\circ} \mathrm{C}$. Second, the Ostwald coefficient is calculated by assuming an 8-fold increase in water solubility with the loss of each $\mathrm{CF}_{2}$. If this holds true, octafluoropropane would have a predicted water solubility of $0.19 \mathrm{~mol} \mathrm{~m}^{-3}$. For comparison, nitrogen has a water solubility of $0.652 \mathrm{~mol} \mathrm{~m}^{-3}$ at $25^{\circ} \mathrm{C}$. This results in a predicted Ostwald coefficient of $540 \times 10^{-6}$. The values of the parameters are listed in Table D.2

In comparison a manufacturer of octafluoropropane lists a water solubility of $0.03 \mathrm{~mol} \mathrm{~m}^{-3}$ (personal communication,ExciMed, Kuala, Lumpur Malaysia). This value, combined with

${ }^{2}$ There appears to be a typo in Table 1 of [103]. The diffusion coefficients were multiplied by $10^{10}$, rather than the listed $10^{9}$ 
Table D.3: Dissolution times

\begin{tabular}{cccc}
\hline Gas & Radius $(\mu \mathrm{m})$ & Ostwald coefficient & Dissolution time $(\mathrm{s})$ \\
\hline \hline Octafluoropropane & 1 & Fitted & 1.7 \\
& & Measured & 15 \\
& 7 & Fitted & 240 \\
Nitrogen & 1 & Measured & 2136 \\
Nitrogen & 7 & & 0.02 \\
& & & 2.8 \\
\hline
\end{tabular}

the saturated vapor pressure [12], results in a calculated Ostwald coefficient of $85.7 \times 10^{-6}$. Again, while saturated vapor pressure at 25 and $37^{\circ} \mathrm{C}$ are known, the solubility at increased water temperature is not known. Therefore the Ostwald coefficient at high temperatures is likely an overestimate of the true value.

The coupled equations were solved with the MATLAB ODE45 numerical solver, a relative tolerance of $1 \times 10^{-4}$, and an absolute tolerance of $1 \times 10^{-7}$. A stopping criteria of $\mid \rho$ $1 \times 10^{-7} \mid<0$ was used. As with the Epstein-Plesset model, non steady-state effects were neglected.

\section{D.2 Results and Discussion}

Dissolution of a pure octafluoropropane microbubble $\left(X_{F}=1\right)$ took on the general form of initial expansion followed by a slower contraction, the rate of which increased as the radius got smaller (Fig. D.1a,b). For a pure nitrogen microbubble $\left(X_{F}=0\right)$, the radius time curve and absolute dissolution time reduced to the simpler Epstein-Plesset model.

A significant difference in dissolution time is obtained depending on the value of the Ostwald coefficient selected (Table D.3). However, the measured OFP Ostwald coefficient appears to be in better agreement with the experimental results (6.4). 


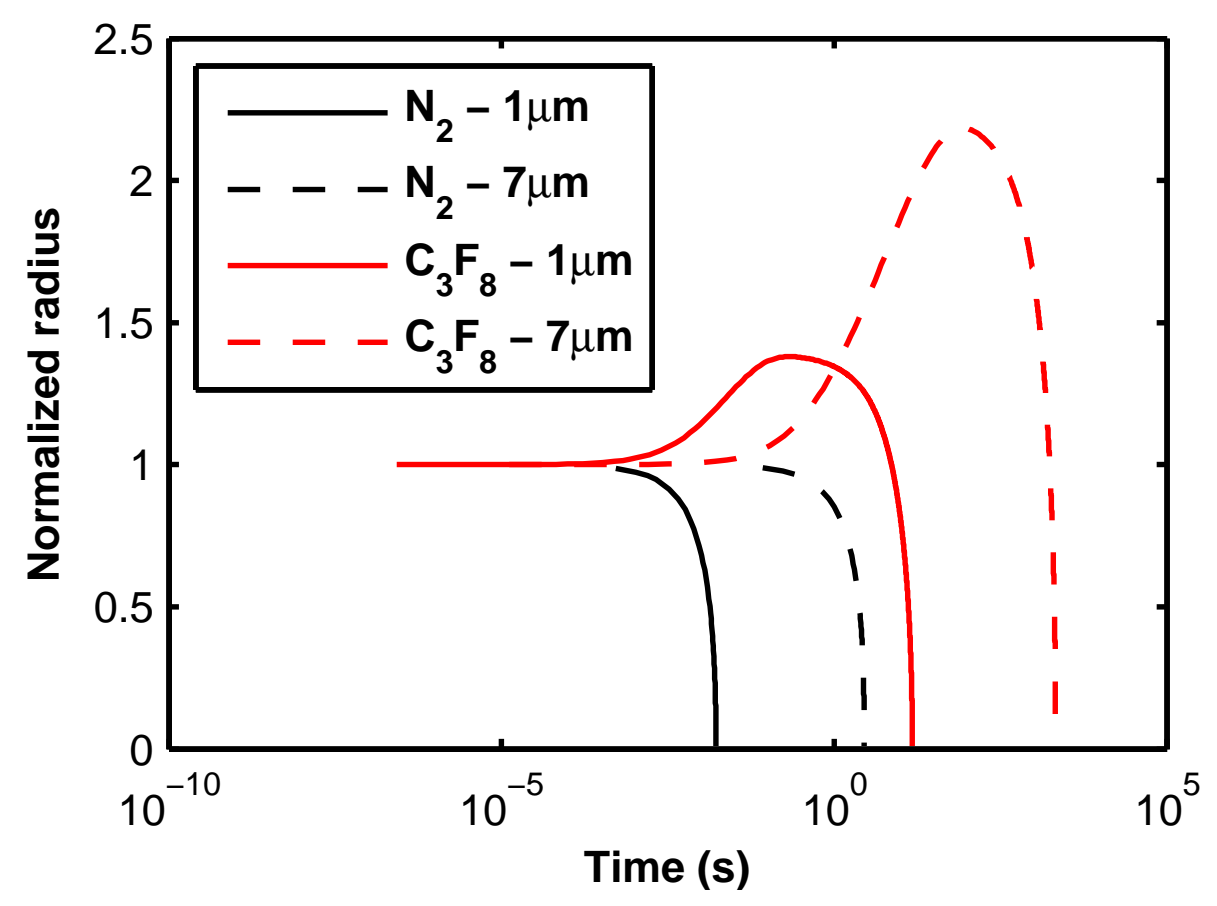

Figure D.1: Normalized radius time curves for microbubbles with a radius of 1 and $7 \mu \mathrm{m}$ and composed of either pure octafluoropropane microbubble $\left(X_{F}=1\right)$ or pure nitrogen microbubble $\left(X_{F}\right.$ $=0)$. Diffusion and Ostwald coefficient properties at $40^{\circ} \mathrm{C}$ were selected. Microbubbles composed of pure fluorocarbon grow then shrink while microbubbles composed solely of nitrogen only shrink. 


\section{Appendix E}

\section{Mass spectrometry of Zonyl (FS-300)}

Zonyl is composed of a mixture of polymers with the general structure:

$$
\mathrm{F}\left(\mathrm{CF}_{2} \mathrm{CF}_{2}\right)_{\mathrm{X}} \mathrm{CH}_{2} \mathrm{CH}_{2} \mathrm{O}\left(\mathrm{CH}_{2} \mathrm{CH}_{2} \mathrm{O}\right)_{\mathrm{Y}} \mathrm{H} \text {, }
$$

where $\mathrm{X}$ is less than 25 and $\mathrm{Y}$ is less than 10 [241]. MALDI mass spectrometry was performed to estimate the molecular weight of the fluorosurfactant (Fig E.1, Table E.1). Only Y values of 5 and 6 were observed. For Y values of 5 , X values of $2-14$ were observed. For Y values of $6, \mathrm{X}$ values of $4-11$ were observed. 
Appendix E | Mass spectrometry of Zonyl (FS-300)

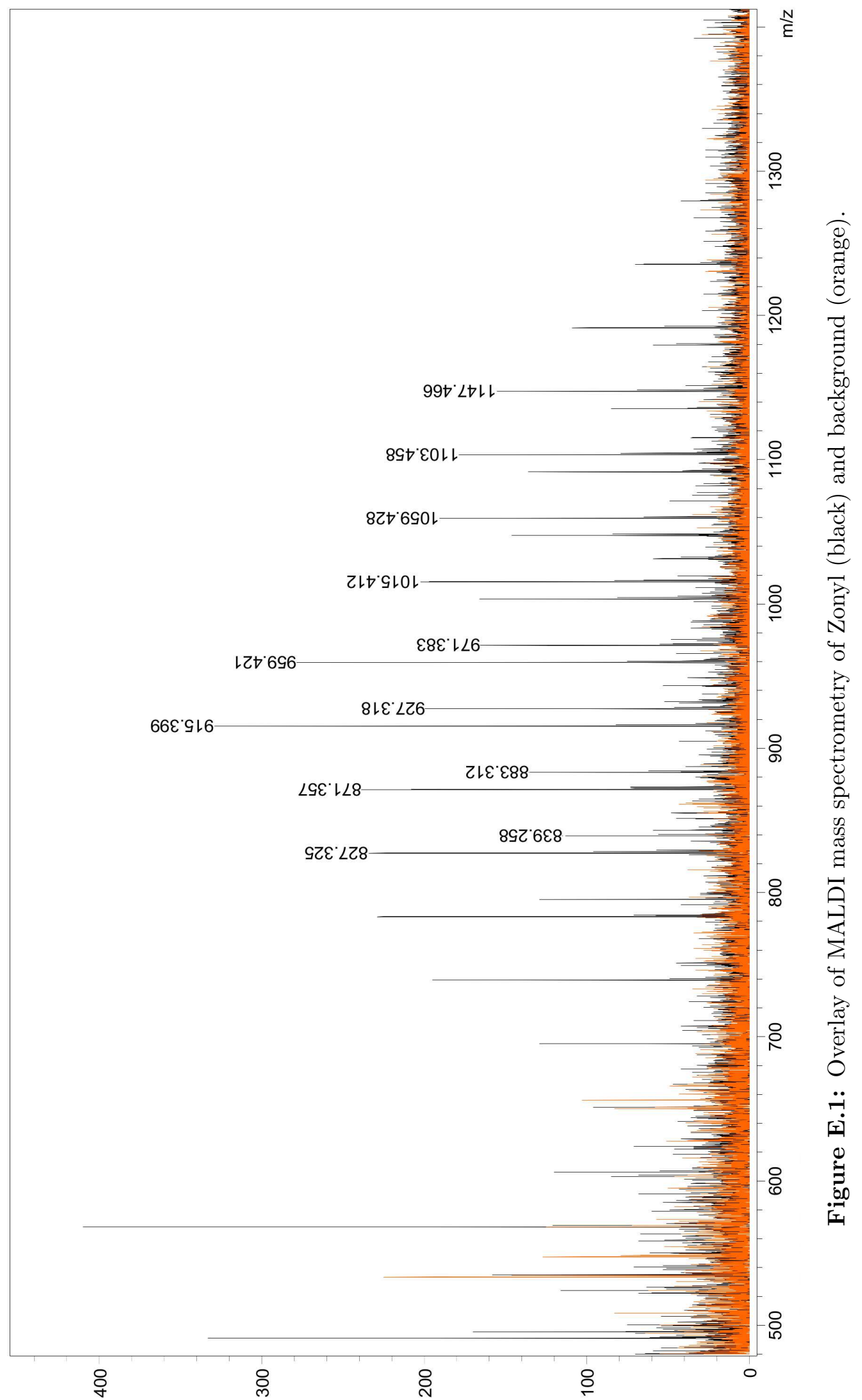

['n'e] 'suәtu। 


\section{Appendix F}

\section{Red blood cell ghost particle loading}

\section{protocol}

There are many variations of the red blood cell ghost protocol, a selection of which include [227, 242, 232, 234, 228, 229, 225, 230]. In its most basic form, red blood cell ghosts are produced by placing red blood cells in a hypotonic solution, to lyse the membrane and allow the hemoglobin to leave, followed by an isotonic solution, to reseal the membrane. The are numerous parameters that can be varied: osmolarity, $\mathrm{pH}$, temperature, buffer, and other salts of the lysing solution; time of incubation; direct incubation versus dialysis in the lysis solution; and osmolarity, $\mathrm{pH}$, temperature, buffer, and other salts of the restoring solution to name a few of the main components. Some variations, such as $\mathrm{KCl}$ or $\mathrm{NaCl}$ as the salt, have little effect, whereas others, such as temperature or osmolarity, can have a large effect. Below I enumerate the current protocol I use to make the microbubble precursors and load the particles into the red blood cells. I have attempted to include notes where appropriate explaining why certain parameters were selected. However, the following protocol should not be considered globally optimized. 


\section{F.1 Microbubble precursor fabrication}

1. $4 \mathrm{~mL}$ of deionized water is placed in a $10 \mathrm{~mL}$ plastic $\mathrm{BD}$ syringe. Whether the water is degassed or not does not appear to affect droplet formation or stability.

2. $50 \mu \mathrm{L}$ of Zonyl (FS300 Sigma-Aldrich) is then added to the water in the syringe. Tween and bovine serum albumin [40] had previously been used to stabilize the particles. However, no change in diameter was observed by DLS and in the absence of either of these surfactants particles still formed. Consequently, it is unclear what effect, if any, these surfactants have on droplet formation. The fluorosurfactant Zonyl was selected under the assumption that it would provide better stabilization as the tail could insert into the liquid phase. As of this writing Sigma-Aldrich has discontinued the sale of FS-300. A straight fluorocarbon-hydrocarbon diblock polymer should also be considered [39].

3. The air is removed from the syringe and replaced with $6 \mathrm{~mL}$ of decafluorobutane. Decafluorobutane was selected as it was hypothesized to provide the right balance of stability, to enable loading, and instability, to enable easy conversion back into a gas. Octafluoropropane was too unstable and could not be converted into stable droplets on its own. Mixtures of decafluorobutane and octafluoropropane were attempted; however, it is unclear whether the liquids truly mixed or phase separated into octafluoropropane droplet and decafluorobutane droplets. Higher boiling point fluorocarbons such as dodecafluoropentane should also be considered.

4. The syringe is then capped with a luer cap (96843 Qosina, Edgewood, NY) and a custom designed clamp is used to compress and hold the syringe (Fig. F.1). At this point large transparent droplets of liquefied decafluorobutane should begin to collect at the bottom of the syringe. 
5. The compressed syringe is then placed in a bath sonicator. Aluminum foil was placed in the bath to determine the acoustic hot spots as evidenced by the area of foil most damaged after $30 \mathrm{~s}$. Care was taken to try and place the syringe in the same place and position each time. The water was pre-cooled in an ice bath and the same volume of water was added each time, ensuring no ice was transferred to the sonicator. Both liquid volume and the presence of ice in the sonicator was observed to effect sonication.

6. The syringe is then sonicated in 10 minute increments. After each increment the water is re-cooled. The color of the solution should become milky white as droplet formation occurs. Progress can also be monitored by checking for large liquid fluorocarbon droplets that have not been broken up.

7. After sonication, pressure is released. If sonication was done correctly, less than $1 \mathrm{~mL}$ of gas conversion should occur. The droplets can then be centrifuged for $60 \mathrm{~s}$ at $1000 \mathrm{RCF}$ to remove the largest of the droplets. The supernatant is then centrifuged for 5 min at $16,000 \mathrm{RCF}$ to pellet the remaining droplets. The particles are resuspended in the lysing solution. Care should be taken not to shake the eppendorf too vigorously when trying to resuspend the particles.

8. Alternatively, the droplets can be fabricated in the lysing solution instead of water, and the red blood cells can be added directly to the syringe.

\section{F.2 Red blood cell ghost loading}

1. EDTA anti-coagulated whole blood was acquired from the UVa clinical laboratory. Since the blood is de-identified, IRB approval is not necessary. The blood acquired from the clinical laboratory is five days old and should be used within a week as older blood loses its ability to reseal. 


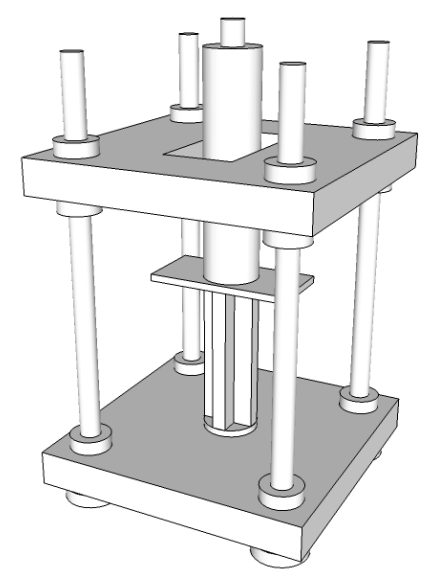

Figure F.1: Syringe holder for maintaining pressure

2. The $0.5 \mathrm{~mL}$ whole blood is washed three times in isotonic saline (0.9\% saline, 07062F7124, Owens \& Minor) for 5 min at 1000 RCF. Care is taken to remove the buffy coat containing white cells at the expense of losing some red cells.

3. $0.2 \mathrm{~mL}$ of the resulting packed red cells are then pre-lysed by adding $1.8 \mathrm{~mL}$ of $0.25 \mathrm{x}$ PBS $+\mathrm{Mg}+\mathrm{Ca}$ (Fisher-Scientific, Pittsburgh, PA), incubating for $5 \mathrm{~min}$ on ice then pelleting via centrifugation for $5 \mathrm{~min}$ at $10,000 \mathrm{RCF}$. This is repeated once more. This step removes the majority of hemoglobin from the cells. The $\mathrm{Mg}$ and $\mathrm{Ca}$ are important to prevent the membrane from vesiculating [234, 229].

4. The lysing solution is composed of $0.01 \mathrm{x}$ PBS without calcium or magnesium but with $0.1 \mathrm{mM}$ EDTA. The pH should be adjusted to 8. Low osmolarity, basic solutions in the absence of divalent cations have been shown to produce large $(1 \mu \mathrm{m})$ diameter pores in the red cell membrane when incubated at $4^{\circ} \mathrm{C}$ for greater than 1 hour. Solutions of $5 \mathrm{mM} \mathrm{Na}_{3} \mathrm{PO}_{4}$ and $5 \mathrm{mM}$ HEPES-Na plus EDTA also appear to work.

5. The pre-lysed cells are combined with the droplets and the lysing solution in a syringe. The syringe is pressured with the same apparatus used to produce the droplets and placed on a rocker overnight at $4^{\circ}$. 
6. After the overnight incubation, a resealing solution consisting of $60 \mu \mathrm{L}$ of $5 \mathrm{M} \mathrm{NaCl}$ and $10 \mu \mathrm{L}$ of $1 \mathrm{M} \mathrm{MgSO}_{4}$ is added to the cells. The cells are then incubated under pressure for $30 \mathrm{~min}$ at $25^{\circ}$. The concentrated $\mathrm{NaCl}$ is to restore isotonicity. The $\mathrm{MgSO}_{4}$ helps prevent additional membrane vesiculation. Increasing the temperature improves membrane resealing and shortens the incubation time. $25^{\circ} \mathrm{C}$ was chosen as a compromise to minimize premature conversion of the fluorocarbon droplets.

7. The red cells are washed three times by centrifugation for 5 min at 7,000 RCF. During centrifugation unencapsulated fluorocarbon droplets form a white pellet underneath the translucent pellet of the red blood cell ghosts. With careful aspiration, it should be possible to separate the ghosts from the droplets. Optimization of centrifugation speeds needs to be performed to ensure that loaded fluorocarbon droplets are not being pulled out of the red blood cell membrane at these high centrifugation speeds.

8. For proof of concept, fluorescent nanometer polystyrene beads can be loaded in place of fluorocarbon droplets. This enables optical confirmation of particle loading. If particles are successfully trapped within the membrane, as opposed to simply being attached to the outer membrane, the particles should move within the red cell yet never be able to escape. Fluorescence is important as many of the ghosts will have entrapped vesicles that may look like a loaded particle (Fig. F.2).

9. When producing droplet loaded ghosts for in vivo use it may be necessary to add glucose, adenosine and pyruvate to the resealing solution [225]. 


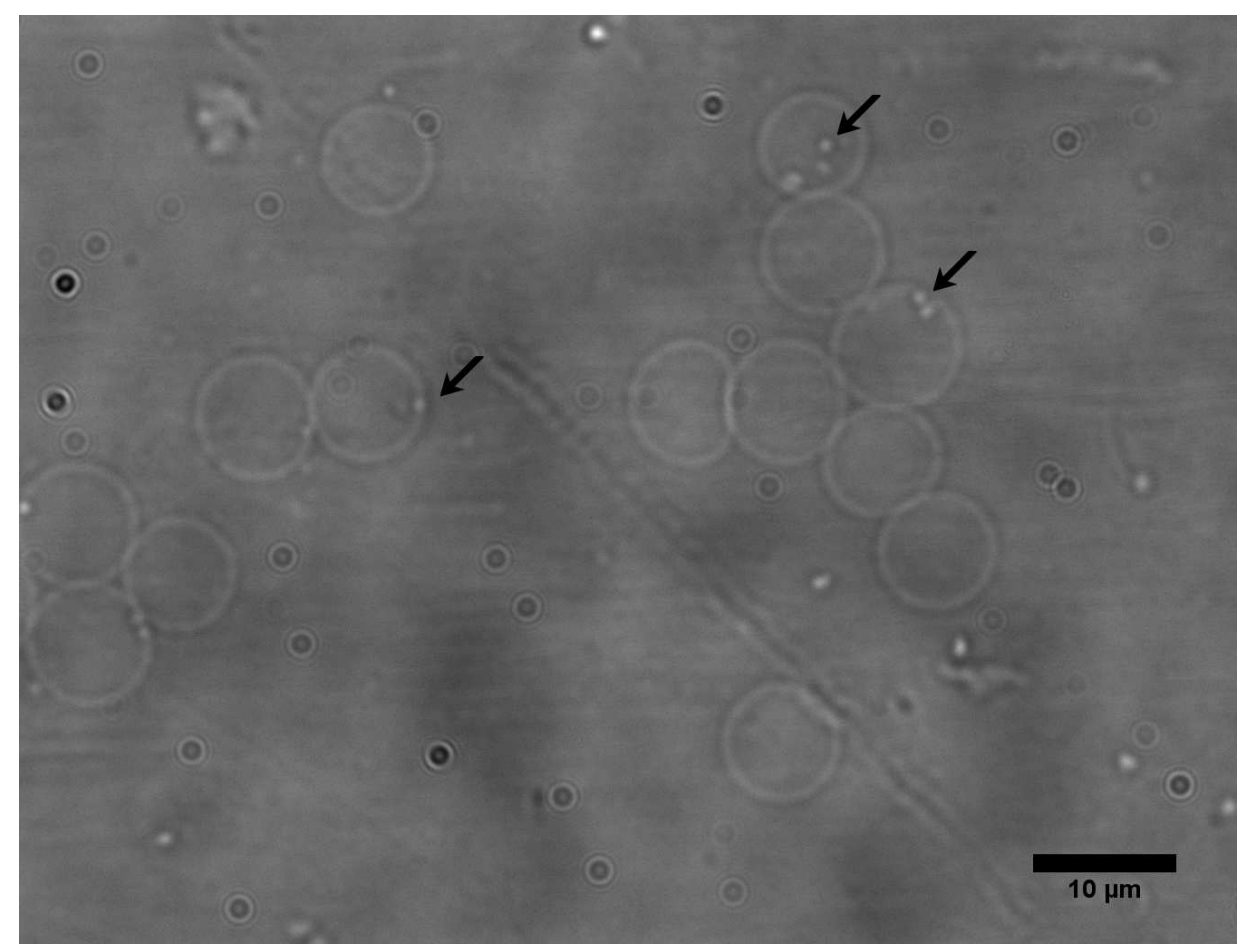

Figure F.2: Brightfield image of red blood cells lysed in resealed in the absence of particles. Arrows point to vesicles outside and inside the cells that may be confused for loaded particles. 


\section{Bibliography}

[1] T. Szabo, "Introduction," in Diagnostic ultrasound imaging : inside out. Burlington MA: Elsevier Academic Press, 2004, pp. 1-27.

[2] H. Routh, "Doppler ultrasound," IEEE Engineering in Medicine and Biology Magazine, vol. 15, no. 6, pp. 31-40, Nov. 1996.

[3] J. Doherty, G. Trahey, K. Nightingale, and M. Palmeri, "Acoustic radiation force elasticity imaging in diagnostic ultrasound," IEEE Transactions on Ultrasonics, Ferroelectrics and Frequency Control, vol. 60, no. 4, pp. -, 2013.

[4] M. Tanter and M. Fink, "Ultrafast imaging in biomedical ultrasound," IEEE Transactions on Ultrasonics, Ferroelectrics and Frequency Control, vol. 61, no. 1, pp. 102119, 2014.

[5] A. E. Sargsyan, D. R. Hamilton, J. A. Jones, S. Melton, P. A. Whitson, A. W. Kirkpatrick, D. Martin, and S. A. Dulchavsky, "FAST at MACH 20: clinical ultrasound aboard the international space station," The Journal of trauma, vol. 58, no. 1, pp. 35-39, Jan. 2005.

[6] M. C. FYFE and M. I. BULLOCK, "Therapeutic ultrasound: Some historical background and development in knowledge of its effect on healing," Australian Journal of Physiotherapy, vol. 31, no. 6, pp. 220-224, 1985.

[7] D. L. Miller, N. B. Smith, M. R. Bailey, G. J. Czarnota, K. Hynynen, and I. R. S. Makin, "Overview of therapeutic ultrasound applications and safety considerations," Journal of Ultrasound in Medicine, vol. 31, no. 4, pp. 623-634, Apr. 2012.

[8] J. C. Gigliotti, L. Huang, H. Ye, A. Bajwa, K. Chattrabhuti, S. Lee, A. L. Klibanov, K. Kalantari, D. L. Rosin, and M. D. Okusa, "Ultrasound prevents renal ischemiareperfusion injury by stimulating the splenic cholinergic anti-inflammatory pathway," Journal of the American Society of Nephrology, Aug. 2013.

[9] A. Maxwell, O. Sapozhnikov, M. Bailey, L. Crum, Z. Xu, B. Fowlkes, C. Cain, and V. Khokhlova, "Disintegration of tissue using high intensity focused ultrasound: Two approaches that utilize shock waves," Acoustics Today, vol. 8, no. 4, pp. 24-37, Oct. 2012. 
[10] D. Schlesinger, S. Benedict, C. Diederich, W. Gedroyc, A. Klibanov, and J. Larner, "MR-guided focused ultrasound surgery, present and future," Medical Physics, vol. 40, no. 8, Aug. 2013.

[11] T. Szabo, "Appendix b," in Diagnostic ultrasound imaging : inside out. Burlington MA: Elsevier Academic Press, 2004, p. 535.

[12] E. Lemmon, M. McLinden, and D. Friend, "Thermophysical properties of fluid systems," in NIST Chemistry WebBook, NIST Standard Reference Database Number 69, 69th ed., P. Linstrom and W. Mallard, Eds. Gaithersburg MD, 20899: National Institute of Standards and Technology, 2014, http://webbook.nist.gov/cgi/cbook.cgi?ID=C7727379\&Mask=10.

[13] R. Gramiak and P. M. Shah, "Echocardiography of the aortic root," Investigative Radiology, vol. 3, no. 5, pp. 356-366, Oct. 1968.

[14] F. W. Kremkau, R. Gramiak, E. L. Carstensen, P. M. Shah, and D. H. Kramer, "Ultrasonic detection of cavitation at catheter tips," The American Journal of Roentgenology, Radium Therapy, and Nuclear Medicine, vol. 110, no. 1, pp. 177-183, Sep. 1970.

[15] M. D. Smith, O. L. Kwan, H. J. Reiser, and A. N. DeMaria, "Superior intensity and reproducibility of SHU-454, a new right heart contrast agent," Journal of the American College of Cardiology, vol. 3, no. 4, pp. 992-998, Apr. 1984.

[16] S. Sirsi and M. Borden, "Microbubble compositions, properties and biomedical applications," Bubble science engineering and technology, vol. 1, no. 1-2, pp. 3-17, Nov. 2009.

[17] A. Klibanov, "Ultrasound contrast agents: Development of the field and current status," in Contrast Agents II, ser. Topics in Current Chemistry, W. Krause, Ed. Springer Berlin / Heidelberg, 2002, vol. 222, pp. 73-106, 10.1007/3-540-46009-8_3.

[18] N. G. Fisher, J. P. Christiansen, A. Klibanov, R. P. Taylor, S. Kaul, and J. R. Lindner, "Influence of microbubble surface charge on capillary transit and myocardial contrast enhancement," Journal of the American College of Cardiology, vol. 40, no. 4, pp. 811-819, Aug. 2002.

[19] K. Yanagisawa, F. Moriyasu, T. Miyahara, M. Yuki, and H. Iijima, "Phagocytosis of ultrasound contrast agent microbubbles by kupffer cells," Ultrasound in Medicine $\&$ Biology, vol. 33, no. 2, pp. 318-325, Feb. 2007.

[20] B. D. Butler and B. A. Hills, "The lung as a filter for microbubbles," Journal of applied physiology: respiratory, environmental and exercise physiology, vol. 47, no. 3, pp. 537-543, Sep. 1979.

[21] R. S. Meltzer, E. Glen Tickner, and R. L. Popp, "Why do the lungs clear ultrasonic contrast?" Ultrasound in Medicine \& Biology, vol. 6, no. 3, pp. 263-269, 1980. 
[22] N. S. Chahal and R. Senior, "Clinical applications of left ventricular opacification," Journal of the American College of Cardiology: Cardiovascular Imaging, vol. 3, no. 2, pp. 188-196, Feb. 2010.

[23] S. R. Wilson and P. N. Burns, "Microbubble-enhanced US in body imaging: What role?" Radiology, vol. 257, no. 1, pp. 24-39, Oct. 2010.

[24] K. Wei, A. R. Jayaweera, S. Firoozan, A. Linka, D. M. Skyba, and S. Kaul, "Quantification of myocardial blood flow with ultrasound-induced destruction of microbubbles administered as a constant venous infusion," Circulation, vol. 97, no. 5, pp. 473-483, Feb. 1998.

[25] P. A. Dayton and J. J. Rychak, "Molecular ultrasound imaging using microbubble contrast agents," Frontiers in Bioscience: A Journal and Virtual Library, vol. 12, pp. 5124-5142, 2007.

[26] R. Gessner and P. A. Dayton, "Advances in molecular imaging with ultrasound," Molecular Imaging, vol. 9, no. 3, pp. 117-127, Jun. 2010.

[27] S. Unnikrishnan and A. L. Klibanov, "Microbubbles as ultrasound contrast agents for molecular imaging: preparation and application," AJR. American journal of roentgenology, vol. 199, no. 2, pp. 292-299, Aug. 2012.

[28] R. Williams, C. Wright, E. Cherin, N. Reznik, M. Lee, I. Gorelikov, F. S. Foster, N. Matsuura, and P. N. Burns, "Characterization of submicron phase-change perfluorocarbon droplets for extravascular ultrasound imaging of cancer," Ultrasound in Medicine ES Biology, vol. 39, no. 3, pp. 475-489, Mar. 2013.

[29] J. M. Gorce, M. Arditi, and M. Schneider, "Influence of bubble size distribution on the echogenicity of ultrasound contrast agents: a study of SonoVue," Investigative radiology, vol. 35, no. 11, pp. 661-671, Nov. 2000.

[30] I. Lentacker, I. De Cock, R. Deckers, S. C. De Smedt, and C. T. W. Moonen, "Understanding ultrasound induced sonoporation: Definitions and underlying mechanisms," Advanced Drug Delivery Reviews, 2013.

[31] K. Ferrara, R. Pollard, and M. Borden, "Ultrasound microbubble contrast agents: fundamentals and application to gene and drug delivery," Annual review of biomedical engineering, vol. 9, pp. 415-447, 2007.

[32] S. Hernot and A. L. Klibanov, "Microbubbles in ultrasound-triggered drug and gene delivery," Advanced Drug Delivery Reviews, vol. 60, no. 10, pp. 1153-1166, Jun. 2008.

[33] M. R. Böhmer, A. L. Klibanov, K. Tiemann, C. S. Hall, H. Gruell, and O. C. Steinbach, "Ultrasound triggered image-guided drug delivery," European Journal of Radiology, vol. 70, no. 2, pp. 242-253, May 2009. 
[34] E. E. Konofagou, Y.-S. Tung, J. Choi, T. Deffieux, B. Baseri, and F. Vlachos, "Ultrasound-induced blood-brain barrier opening," Current pharmaceutical biotechnology, vol. 13, no. 7, pp. 1332-1345, Jun. 2012.

[35] T. R. Porter and F. Xie, "Ultrasound, microbubbles, and thrombolysis," Progress in Cardiovascular Diseases, vol. 44, no. 2, pp. 101-110, Sep. 2001.

[36] S. Umemura, K. Kawabata, and K. Sasaki, "In vivo acceleration of ultrasonic tissue heating by microbubble agent," IEEE Transactions on Ultrasonics, Ferroelectrics and Frequency Control, vol. 52, no. 10, pp. 1690-1698, Oct. 2005.

[37] L. Phillips, A. Klibanov, B. Wamhoff, and J. Hossack, "Ultrasound-microbubblemediated drug delivery efficacy and cell viability depend on microbubble radius and ultrasound frequency," in 2010 IEEE Ultrasonics Symposium (IUS), 2010, pp. 17751778 .

[38] J. J. Choi, J. A. Feshitan, B. Baseri, S. Wang, Y.-S. Tung, M. A. Borden, and E. E. Konofagou, "Microbubble-size dependence of focused ultrasound-induced bloodbrain barrier opening in mice in vivo," IEEE transactions on bio-medical engineering, vol. 57, no. 1, pp. 145-154, Jan. 2010.

[39] J. G. Riess and M. P. Krafft, "Fluorocarbon emulsions as in vivo oxygen delivery systems: Background and chemistry," in Blood Substitutes, R. M. Winslow, Ed. Academic Press, Oct. 2005, pp. 259-275.

[40] O. D. Kripfgans, J. Fowlkes, D. L. Miller, O. Eldevik, and P. L. Carson, "Acoustic droplet vaporization for therapeutic and diagnostic applications," Ultrasound in Medicine $\mathscr{E}$ Biology, vol. 26, no. 7, pp. 1177-1189, Sep. 2000.

[41] D. Bardin, T. D. Martz, P. S. Sheeran, R. Shih, P. A. Dayton, and A. P. Lee, "Highspeed, clinical-scale microfluidic generation of stable phase-change droplets for gas embolotherapy," Lab on a Chip, vol. 11, no. 23, p. 3990, 2011.

[42] K. Wilson, K. Homan, and S. Emelianov, "Biomedical photoacoustics beyond thermal expansion using triggered nanodroplet vaporization for contrast-enhanced imaging," Nature Communications, vol. 3, p. 618, Jan. 2012.

[43] P. S. Sheeran, J. E. Streeter, L. B. Mullin, T. O. Matsunaga, and P. A. Dayton, "Toward ultrasound molecular imaging with phase-change contrast agents: An in vitro proof of principle," Ultrasound in Medicine E Biology, vol. 39, no. 5, pp. 893-902, May 2013.

[44] O. Shpak, M. Verweij, H. J. Vos, N. d. Jong, D. Lohse, and M. Versluis, "Acoustic droplet vaporization is initiated by superharmonic focusing," Proceedings of the National Academy of Sciences, p. 201312171, Jan. 2014.

[45] S. Jafari, O. Diou, J. Mamou, G. Renault, E. Fattal, N. Tsapis, and S. Bridal, "Highfrequency (20 to $40 \mathrm{MHz}$ ) acoustic response of liquid-filled nanocapsules," IEEE 
Transactions on Ultrasonics, Ferroelectrics and Frequency Control, vol. 61, no. 1, pp. 5-15, Jan. 2014.

[46] E. Stride and M. Edirisinghe, "Novel preparation techniques for controlling microbubble uniformity: a comparison," Medical \& Biological Engineering \& Computing, vol. 47, no. 8, pp. 883-892, Aug. 2009.

[47] A. M. Gañán-Calvo and J. M. Gordillo, "Perfectly monodisperse microbubbling by capillary flow focusing," Physical Review Letters, vol. 87, no. 27 Pt 1, p. 274501, Dec. 2001.

[48] P. Garstecki, I. Gitlin, W. DiLuzio, G. M. Whitesides, E. Kumacheva, and H. A. Stone, "Formation of monodisperse bubbles in a microfluidic flow-focusing device," Applied Physics Letters, vol. 85, no. 13, pp. 2649-2651, Sep. 2004.

[49] J. M. Gordillo, Z. Cheng, A. M. Ganan-Calvo, M. Márquez, and D. A. Weitz, "A new device for the generation of microbubbles," Physics of Fluids, vol. 16, no. 8, p. $2828,2004$.

[50] E. Talu, M. M. Lozano, R. L. Powell, P. A. Dayton, and M. L. Longo, "Long-term stability by lipid coating monodisperse microbubbles formed by a flow-focusing device," Langmuir, vol. 22, no. 23, pp. 9487-9490, Nov. 2006.

[51] K. Hettiarachchi, E. Talu, M. L. Longo, P. A. Dayton, and A. P. Lee, "On-chip generation of microbubbles as a practical technology for manufacturing contrast agents for ultrasonic imaging," Lab on a Chip, vol. 7, no. 4, pp. 463-468, Apr. 2007.

[52] E. Castro-Hernández, W. van Hoeve, D. Lohse, and J. M. Gordillo, "Microbubble generation in a co-flow device operated in a new regime," Lab on a Chip, vol. 11, no. 12, pp. 2023-2029, Jun. 2011.

[53] P. Garstecki, M. J. Fuerstman, H. A. Stone, and G. M. Whitesides, "Formation of droplets and bubbles in a microfluidic t-junction-scaling and mechanism of breakup," Lab Chip, vol. 6, no. 3, pp. 437-446, Feb. 2006.

[54] O. Gunduz, Z. Ahrnad, E. Stride, and M. Edirisinghe, "A device for the fabrication of multifunctional particles from microbubble suspensions," Materials Science \&6 Engineering C-Materials for Biological Applications, vol. 32, no. 4, pp. 1005-1010, May 2012.

[55] U. Farook, H. B. Zhang, M. J. Edirisinghe, E. Stride, and N. Saffari, "Preparation of microbubble suspensions by co-axial electrohydrodynamic atomization," Medical engineering 85 physics, vol. 29, no. 7, pp. 749-754, Sep. 2007.

[56] T. Cubaud, M. Tatineni, X. Zhong, and C.-M. Ho, "Bubble dispenser in microfluidic devices," Physical Review. E, Statistical, Nonlinear, and Soft Matter Physics, vol. 72, no. 3 Pt 2, p. 037302, Sep. 2005. 
[57] D. C. Duffy, J. C. McDonald, O. J. Schueller, and G. M. Whitesides, "Rapid prototyping of microfluidic systems in poly(dimethylsiloxane)," Analytical chemistry, vol. 70, no. 23, pp. 4974-4984, Dec. 1998.

[58] J. C. McDonald, D. C. Duffy, J. R. Anderson, D. T. Chiu, H. Wu, O. J. Schueller, and G. M. Whitesides, "Fabrication of microfluidic systems in poly(dimethylsiloxane)," Electrophoresis, vol. 21, no. 1, pp. 27-40, Jan. 2000.

[59] Y.-C. Tan, V. Cristini, and A. P. Lee, "Monodispersed microfluidic droplet generation by shear focusing microfluidic device," Sensors and Actuators B: Chemical, vol. 114, no. 1, pp. 350-356, Mar. 2006.

[60] H. C. Starritt, F. A. Duck, and V. F. Humphrey, "Forces acting in the direction of propagation in pulsed ultrasound fields," Physics in Medicine and Biology, vol. 36, no. 11, p. 1465, Nov. 1991.

[61] A. A. Doinikov, "Bjerknes forces and translational bubble dynamics," in Bubble and Particle Dynamics in Acoustic Fields: Modern Trends and Applications, A. Doinikov, Ed. Research Signpost, 2005, pp. 95-143.

[62] J. J. Rychak, A. L. Klibanov, and J. A. Hossack, "Acoustic radiation force enhances targeted delivery of ultrasound contrast microbubbles: in vitro verification," IEEE Transactions on Ultrasonics, Ferroelectrics, and Frequency Control, vol. 52, no. 3, pp. 421-433, Mar. 2005.

[63] M. Shortencarier, P. Dayton, S. H. Bloch, P. Schumann, T. Matsunaga, and K. Ferrara, "A method for radiation-force localized drug delivery using gas-filled lipospheres," IEEE Transactions on Ultrasonics, Ferroelectrics and Frequency Control, vol. 51, no. 7, pp. 822-831, 2004.

[64] J. Kilroy, A. Klibanov, D. K. Bowles, B. R. Wamhoff, and J. A. Hossack, "Localized in vivo model drug delivery with intravascular ultrasound and microbubbles," Ultrasound in Medicine \& Biology, vol. In press, 2014.

[65] S. L. Mulvagh, A. N. DeMaria, S. B. Feinstein, P. N. Burns, S. Kaul, J. G. Miller, M. Monaghan, T. R. Porter, L. J. Shaw, and F. S. Villanueva, "Contrast echocardiography: Current and future applications," Journal of the American Society of Echocardiography, vol. 13, no. 4, pp. 331-342, Apr. 2000.

[66] M. F. Meloni, T. Livraghi, C. Filice, S. Lazzaroni, F. Calliada, and L. Perretti, "Radiofrequency ablation of liver tumors: the role of microbubble ultrasound contrast agents," Ultrasound Quarterly, vol. 22, no. 1, pp. 41-47, Mar. 2006.

[67] T. A. Fritz, E. C. Unger, G. Sutherland, and D. Sahn, "Phase i clinical trials of MRX-115. a new ultrasound contrast agent," Investigative Radiology, vol. 32, no. 12, pp. 735-740, Dec. 1997. 
[68] M. W. Keller, S. B. Feinstein, and D. D. Watson, "Successful left ventricular opacification following peripheral venous injection of sonicated contrast agent: an experimental evaluation," American heart journal, vol. 114, no. 3, pp. 570-575, Sep. 1987.

[69] U. Farook, E. Stride, and M. J. Edirisinghe, "Preparation of suspensions of phospholipid-coated microbubbles by coaxial electrohydrodynamic atomization," Journal of the Royal Society, Interface / the Royal Society, vol. 6, no. 32, pp. 271277, Mar. 2009.

[70] M. Kaya, S. Feingold, K. Hettiarachchi, A. P. Lee, and P. A. Dayton, "Acoustic responses of monodisperse lipid-encapsulated microbubble contrast agents produced by flow focusing," Bubble Science Engineering and Technology, vol. 2, no. 2, pp. 3340, Dec. 2010.

[71] E. Talu, K. Hettiarachchi, S. Zhao, R. L. Powell, A. P. Lee, M. L. Longo, and P. A. Dayton, "Tailoring the size distribution of ultrasound contrast agents: possible method for improving sensitivity in molecular imaging," Molecular Imaging, vol. 6, no. 6, pp. 384-392, Dec. 2007.

[72] J. A. Feshitan, C. C. Chen, J. J. Kwan, and M. A. Borden, "Microbubble size isolation by differential centrifugation," Journal of Colloid and Interface Science, vol. 329, no. 2, pp. 316-324, Jan. 2009.

[73] D. Huh, J. H. Bahng, Y. Ling, H.-H. Wei, O. D. Kripfgans, J. B. Fowlkes, J. B. Grotberg, and S. Takayama, "Gravity-driven microfluidic particle sorting device with hydrodynamic separation amplification," Analytical Chemistry, vol. 79, no. 4, pp. 1369-1376, Feb. 2007.

[74] S. Kapishnikov, V. Kantsler, and V. Steinberg, "Continuous particle size separation and size sorting using ultrasound in a microchannel," Journal of Statistical Mechanics: Theory and Experiment, vol. 2006, no. 01, pp. P01 012-P01 012, Jan. 2006.

[75] S. Kvåle, H. A. Jakobsen, O. A. Asbjornsen, and T. Omtveit, "Size fractionation of gas-filled microspheres by flotation," Separations Technology, vol. 6, no. 4, pp. 219-226, Oct. 1996.

[76] O. Gunduz, Z. Ahmad, E. Stride, C. Tamerler, and M. Edirisinghe, "Bioinspired bubble design for particle generation," Journal of the Royal Society Interface, vol. 9, no. 67, pp. 389-395, Feb. 2012.

[77] W. J. Duncanson, A. Abbaspourrad, H. C. Shum, S.-H. Kim, L. L. A. Adams, and D. A. Weitz, "Monodisperse gas-filled microparticles from reactions in double emulsions," Langmuir, vol. 28, no. 17, pp. 6742-6745, May 2012.

[78] Lantheus Medical, "Definity (package insert)," 2008.

[79] H. Iijima, F. Moriyasu, T. Miyahara, and K. Yanagisawa, "Ultrasound contrast agent, levovist microbubbles are phagocytosed by kupffer cells-in vitro and in vivo 
studies," Hepatology research: the official journal of the Japan Society of Hepatology, vol. 35, no. 4, pp. 235-237, Aug. 2006.

[80] A. K. P. Lim, N. Patel, R. J. Eckersley, S. D. Taylor-Robinson, D. O. Cosgrove, and M. J. K. Blomley, "Evidence for spleen-specific uptake of a microbubble contrast agent: A quantitative study in healthy volunteers," Radiology, vol. 231, no. 3, pp. 785-788, Jun. 2004.

[81] E. Talu, R. L. Powell, M. L. Longo, and P. A. Dayton, "Needle size and injection rate impact microbubble contrast agent population," Ultrasound in Medicine $\mathscr{G}$ Biology, vol. 34, no. 7, pp. 1182-1185, Jul. 2008.

[82] B. B. Goldberg, J.-B. Liu, and F. Forsberg, "Ultrasound contrast agents: A review," Ultrasound in Medicine \& Biology, vol. 20, no. 4, pp. 319-333, 1994.

[83] K. Hettiarachchi, S. Zhang, S. Feingold, A. P. Lee, and P. A. Dayton, "Controllable microfluidic synthesis of multiphase drug-carrying lipospheres for site-targeted therapy," Biotechnology Progress, vol. 25, no. 4, pp. 938-945, Aug. 2009.

[84] V. Barbier, H. Willaime, P. Tabeling, and F. Jousse, "Producing droplets in parallel microfluidic systems," Physical Review E, vol. 74, no. 4, p. 046306, Oct. 2006.

[85] M. Hashimoto, S. S. Shevkoplyas, B. Zasońska, T. Szymborski, P. Garstecki, and G. M. Whitesides, "Formation of bubbles and droplets in parallel, coupled flowfocusing geometries," Small (Weinheim an Der Bergstrasse, Germany), vol. 4, no. 10, pp. 1795-1805, Oct. 2008.

[86] K. Hettiarachchi, E. Talu, M. L. Longo, P. A. Dayton, and A. P. Lee, "Multi-array flow-focusing devices to accelerate production of microbubbles for contrast-enhanced ultrasound imaging," in Eleventh International Conference on Miniaturized Systems for Chemistry and Life Sciences, Paris, France, Oct. 2007, pp. 664-666.

[87] W. Li, E. W. K. Young, M. Seo, Z. Nie, P. Garstecki, C. A. Simmons, and E. Kumacheva, "Simultaneous generation of droplets with different dimensions in parallel integrated microfluidic droplet generators," Soft Matter, vol. 4, no. 2, pp. 258-262, Jan. 2008.

[88] M. K. Mulligan and J. P. Rothstein, "Scale-up and control of droplet production in coupled microfluidic flow-focusing geometries," Microfluidics and Nanofluidics, vol. 13 , no. 1 , pp. $65-73$, Jul. 2012.

[89] J. C. Love, J. R. Anderson, and G. M. Whitesides, "Fabrication of three-dimensional microfluidic systems by soft lithography," MRS Bulletin, vol. 26, no. 07, pp. 523-528, 2001.

[90] L. D. Palma and M. Bertolotto, "Introduction to ultrasound contrast agents: physics overview," European Radiology, vol. 9, no. 3, pp. S338-S342, Nov. 1999. 
[91] P. M. Morse and K. U. Ingard, Theoretical Acoustics. Princeton, NJ: Princeton University Press, 1968.

[92] R. Y. Nishi, "Ultrasonic detection of bubbles with doppler flow transducers," Ultrasonics, vol. 10, no. 4, pp. 173-179, Jul. 1972.

[93] C. Jiang, X. Li, F. Yan, Z. Wang, Q. Jin, F. Cai, M. Qian, X. Liu, L. Zhang, and H. Zheng, "Microfluidic-assisted formation of multifunctional monodisperse microbubbles for diagnostics and therapeutics," Micro $\&$ Nano Letters, vol. 6, no. 6, pp. 417-421, Jun. 2011.

[94] R. Lo and E. Meng, "Integrated and reusable in-plane microfluidic interconnects," Sensors and Actuators B-Chemical, vol. 132, no. 2, pp. 531-539, Jun. 2008.

[95] C. K. Fredrickson and Z. H. Fan, "Macro-to-micro interfaces for microfluidic devices," Lab on a Chip, vol. 4, no. 6, pp. 526-533, Nov. 2004.

[96] J.-K. Lee, M. C. Kung, H. H. Kung, and L. F. Mockros, "Microchannel technologies for artificial lungs: (3) open rectangular channels," ASAIO Journal (American Society for Artificial Internal Organs: 1992), vol. 54, no. 4, pp. 390-395, Aug. 2008.

[97] J. Ryley and O. M. Pereira-Smith, "Microfluidics device for single cell gene expression analysis in saccharomyces cerevisiae," Yeast (Chichester, England), vol. 23, no. 14-15, pp. 1065-1073, Nov. 2006.

[98] M.-E. Vlachopoulou, A. Tserepi, P. Pavli, P. Argitis, M. Sanopoulou, and K. Misiakos, "A low temperature surface modification assisted method for bonding plastic substrates," Journal of Micromechanics and Microengineering, vol. 19, no. 1, p. 015007, Jan. 2009.

[99] P. Phillips, "Contrast pulse sequences (CPS): imaging nonlinear microbubbles," in IEEE Ultrasonics Symposium, vol. 2, Atlanta, GA, 2001, pp. 1739 -1745 vol.2.

[100] P. Guillot and A. Colin, "Stability of parallel flows in a microchannel after a t junction," Physical Review E, vol. 72, no. 6, p. 066301, Dec. 2005.

[101] J. H. Xu, S. W. Li, Y. J. Wang, and G. S. Luo, "Controllable gas-liquid phase flow patterns and monodisperse microbubbles in a microfluidic t-junction device," Applied Physics Letters, vol. 88, no. 13, p. 133506, Mar. 2006.

[102] J. Kilroy, A. Patil, J. Rychak, and J. Hossack, "An IVUS transducer for microbubble therapies," IEEE Transactions on Ultrasonics, Ferroelectrics and Frequency Control, vol. 61, no. 3, pp. 441-449, Mar. 2014.

[103] A. Kabalnov, D. Klein, T. Pelura, E. Schutt, and J. Weers, "Dissolution of multicomponent microbubbles in the bloodstream: 1. theory," Ultrasound in Medicine $\&$ Biology, vol. 24, no. 5, pp. 739-749, Jun. 1998. 
[104] D. K. Wood, S.-H. Oh, S.-H. Lee, H. T. Soh, and A. N. Cleland, "High-bandwidth radio frequency coulter counter," Applied Physics Letters, vol. 87, no. 18, pp. 184 106-184 106-3, Oct. 2005.

[105] H. Zhang, C. Chon, X. Pan, and D. Li, "Methods for counting particles in microfluidic applications," Microfluidics and Nanofluidics, vol. 7, no. 6, pp. 739-749, 2009.

[106] A. M. Gañán-Calvo, "Perfectly monodisperse microbubbling by capillary flow focusing: an alternate physical description and universal scaling," Physical Review. E, Statistical, Nonlinear, and Soft Matter Physics, vol. 69, no. 2 Pt 2, p. 027301, Feb. 2004.

[107] E. Stride and M. Edirisinghe, "Novel microbubble preparation technologies," Soft Matter, vol. 4, p. 2350, 2008.

[108] J. M. Gordillo, A. Sevilla, and C. Martínez-Bazán, "Bubbling in a co-flow at high reynolds numbers," Physics of Fluids, vol. 19, no. 7, pp. 077 102-077102-18, Jul. 2007.

[109] J. M. Gordillo, A. M. Gañán-Calvo, and M. Pérez-Saborid, "Monodisperse microbubbling: Absolute instabilities in coflowing gas-liquid jets," Physics of Fluids, vol. 13, no. 12, pp. 3839-3842, Dec. 2001.

[110] H. N. Oguz and A. Prosperetti, "Dynamics of bubble growth and detachment from a needle," Journal of Fluid Mechanics, vol. 257, pp. 111-145, 1993.

[111] P. Garstecki, H. A. Stone, and G. M. Whitesides, "Mechanism for flow-rate controlled breakup in confined geometries: a route to monodisperse emulsions," Physical Review Letters, vol. 94, no. 16, p. 164501, Apr. 2005.

[112] P. Garstecki and G. M. Whitesides, "Formation of bubbles and droplets in microfluidic systems," Bulletin Of The Polish Academy Of Sciences Technical Sciences, vol. 53, no. 4, p. 361-372, 2005.

[113] P. Garstecki, M. J. Fuerstman, and G. M. Whitesides, "Nonlinear dynamics of a flow-focusing bubble generator: An inverted dripping faucet," Physical Review Letters, vol. 94, no. 23, p. 234502, Jun. 2005.

[114] R. Xiong, M. Bai, and J. N. Chung, "Formation of bubbles in a simple co-flowing micro-channel," Journal of Micromechanics and Microengineering, vol. 17, no. 5, pp. 1002-1011, May 2007.

[115] D. J. Beebe, G. A. Mensing, and G. M. Walker, "Physics and applications of microfluidics in biology," Annual Review of Biomedical Engineering, vol. 4, no. 1, pp. 261-286, Aug. 2002.

[116] S. A. Peyman, R. H. Abou-Saleh, J. R. McLaughlan, N. Ingram, B. R. G. Johnson, K. Critchley, S. Freear, J. A. Evans, A. F. Markham, P. L. Coletta, and S. D. Evans, "Expanding 3D geometry for enhanced on-chip microbubble production and single 
step formation of liposome modified microbubbles," Lab on a chip, vol. 12, no. 21, pp. 4544-4552, Nov. 2012.

[117] M. R. Kendall, D. Bardin, R. Shih, P. A. Dayton, and A. P. Lee, "Scaled-up production of monodisperse, dual layer microbubbles using multi-array microfluidic module for medical imaging and drug delivery," Bubble science engineering and technology, vol. 4, no. 1, pp. 12-20, May 2012.

[118] E. Talu, K. Hettiarachchi, R. L. Powell, A. P. Lee, P. A. Dayton, and M. L. Longo, "Maintaining monodispersity in a microbubble population formed by flow-focusing," Langmuir: the ACS journal of surfaces and colloids, vol. 24, no. 5, pp. 1745-1749, Mar. 2008.

[119] T. Fu, Y. Ma, D. Funfschilling, and H. Z. Li, "Bubble formation and breakup mechanism in a microfluidic flow-focusing device," Chemical Engineering Science, vol. 64, no. 10, pp. 2392-2400, May 2009.

[120] N. Dietrich, S. Poncin, N. Midoux, and H. Z. Li, "Bubble formation dynamics in various flow-focusing microdevices," Langmuir, vol. 24, no. 24, pp. 13904-13911, 2008.

[121] J. J. Kwan and M. A. Borden, "Microbubble dissolution in a multigas environment," Langmuir, vol. 26, no. 9, pp. 6542-6548, May 2010.

[122] M. W. Keller, W. Glasheen, and S. Kaul, "Albunex: a safe and effective commercially produced agent for myocardial contrast echocardiography," Journal of the American Society of Echocardiography: official publication of the American Society of Echocardiography, vol. 2, no. 1, pp. 48-52, Feb. 1989.

[123] J.-L. Vincent, M. M. Wilkes, and R. J. Navickis, "Safety of human albumin-serious adverse events reported worldwide in 1998-2000," British journal of anaesthesia, vol. 91, no. 5, pp. 625-630, Nov. 2003.

[124] M. W. Grinstaff and K. S. Suslick, "Air-filled proteinaceous microbubbles: synthesis of an echo-contrast agent," Proceedings of the National Academy of Sciences of the United States of America, vol. 88, no. 17, pp. 7708-7710, Sep. 1991.

[125] A. Krishnan, J. Sturgeon, C. A. Siedlecki, and E. A. Vogler, "Scaled interfacial activity of proteins at the liquid-vapor interface," Journal of biomedical materials research. Part A, vol. 68, no. 3, pp. 544-557, Mar. 2004.

[126] S. Avivi and A. Gedanken, "S-s bonds are not required for the sonochemical formation of proteinaceous microspheres: the case of streptavidin," The Biochemical journal, vol. 366, no. Pt 3, pp. 705-707, Sep. 2002.

[127] D. Ballard, "Generalizing the hough transform to detect arbitrary shapes," Pattern Recognition, vol. 13, no. 2, pp. 111-122, 1981, WOS:A1981LH42300002. 
[128] T. Sanada, M. Watanabe, and T. Fukano, "Effects of viscosity on coalescence of a bubble upon impact with a free surface," Chemical Engineering Science, vol. 60, no. 19, pp. 5372-5384, Sep. 2005.

[129] E. Talu, K. Hettiarachchi, H. Nguyen, A. Lee, R. Powell, M. Longo, and P. Dayton, "P2A-8 lipid-stabilized monodisperse microbubbles produced by flow focusing for use as ultrasound contrast agents," in IEEE Ultrasonics Symposium, 2006, Vancouver, BC, Canada, 2006, pp. 1568-1571.

[130] L. Chen, Y. Li, and R. Manasseh, "The coalescence of bubbles-a numerical study. third international conference on multiphase," in Third International Conference on Multiphase Flow, 1998, pp. 8-12.

[131] T. R. Porter, F. Xie, J. R. Anderson, A. Kricsfeld, and A. D'Sa, "Multifold sonicated dilutions of albumin with fifty percent dextrose improve left ventricular contrast videointensity after intravenous injection in human beings," Journal of the American Society of Echocardiography: official publication of the American Society of Echocardiography, vol. 7, no. 5, pp. 465-471, Oct. 1994.

[132] R. J. Browning, H. Mulvana, M.-X. Tang, J. V. Hajnal, D. J. Wells, and R. J. Eckersley, "Effect of albumin and dextrose concentration on ultrasound and microbubble mediated gene transfection in vivo," Ultrasound in medicine $\mathscr{G}$ biology, vol. 38, no. 6, pp. 1067-1077, Jun. 2012.

[133] H. Hellebust, C. Christiansen, and T. Skotland, "Biochemical characterization of air-filled albumin microspheres," Biotechnology and applied biochemistry, vol. 18 ( Pt 3), pp. 227-237, Dec. 1993.

[134] GE Healthcare, "Optison (package insert)," 2012.

[135] H. Medwin, "Counting bubbles acoustically: a review," Ultrasonics, vol. 15, no. 1, pp. 7-13, Jan. 1977.

[136] J. E. Streeter, R. Gessner, I. Miles, and P. A. Dayton, "Improving sensitivity in ultrasound molecular imaging by tailoring contrast agent size distribution: in vivo studies," Molecular imaging, vol. 9, no. 2, pp. 87-95, Apr. 2010.

[137] Z. Fan, H. Liu, M. Mayer, and C. X. Deng, "Spatiotemporally controlled single cell sonoporation," Proceedings of the National Academy of Sciences, vol. 109, no. 41, pp. 16 486-16 491, Oct. 2012.

[138] M. Fechheimer, J. F. Boylan, S. Parker, J. E. Sisken, G. L. Patel, and S. G. Zimmer, "Transfection of mammalian cells with plasmid DNA by scrape loading and sonication loading." Proceedings of the National Academy of Sciences of the United States of America, vol. 84, no. 23, pp. 8463-8467, Dec. 1987.

[139] S. Bao, B. D. Thrall, and D. L. Miller, "Transfection of a reporter plasmid into cultured cells by sonoporation in vitro," Ultrasound in Medicine $\&$ Biology, vol. 23, no. 6, pp. 953-959, 1997. 
[140] H. R. Guzmán, D. X. Nguyen, S. Khan, and M. R. Prausnitz, "Ultrasound-mediated disruption of cell membranes. i. quantification of molecular uptake and cell viability," The Journal of the Acoustical Society of America, vol. 110, no. 1, pp. 588-596, Jul. 2001.

[141] I. V. Larina, B. M. Evers, and R. O. Esenaliev, "Optimal drug and gene delivery in cancer cells by ultrasound-induced cavitation," Anticancer research, vol. 25, no. 1A, pp. 149-156, Feb. 2005.

[142] S. Mehier-Humbert, T. Bettinger, F. Yan, and R. H. Guy, "Plasma membrane poration induced by ultrasound exposure: implication for drug delivery," Journal of controlled release: official journal of the Controlled Release Society, vol. 104, no. 1, pp. 213-222, May 2005.

[143] D. M. Hallow, A. D. Mahajan, T. E. McCutchen, and M. R. Prausnitz, "Measurement and correlation of acoustic cavitation with cellular bioeffects," Ultrasound in medicine $\mathscr{E}$ biology, vol. 32, no. 7, pp. 1111-1122, Jul. 2006.

[144] M. A. Hassan, M. A. Buldakov, R. Ogawa, Q.-L. Zhao, Y. Furusawa, N. Kudo, T. Kondo, and P. Riesz, "Modulation control over ultrasound-mediated gene delivery: evaluating the importance of standing waves," Journal of controlled release: official journal of the Controlled Release Society, vol. 141, no. 1, pp. 70-76, Jan. 2010.

[145] L. C. Phillips, A. L. Klibanov, B. R. Wamhoff, and J. A. Hossack, "Localized ultrasound enhances delivery of rapamycin from microbubbles to prevent smooth muscle proliferation," Journal of Controlled Release, vol. 154, no. 1, pp. 42-49, Aug. 2011.

[146] L. C. Phillips, A. L. Klibanov, D. K. Bowles, M. Ragosta, J. A. Hossack, and B. R. Wamhoff, "Focused in vivo delivery of plasmid DNA to the porcine vascular wall via intravascular ultrasound destruction of microbubbles," Journal of Vascular Research, vol. 47 , no. 3, pp. 270-274, 2010.

[147] L. C. Phillips, A. H. Dhanaliwala, A. L. Klibanov, J. A. Hossack, and B. R. Wamhoff, "Focused ultrasound-mediated drug delivery from microbubbles reduces drug dose necessary for therapeutic effect on neointima formation-brief report," Arteriosclerosis, Thrombosis, and Vascular Biology, vol. 31, no. 12, pp. 2853-2855, Dec. 2011.

[148] A. V. Patil, J. J. Rychak, J. S. Allen, A. L. Klibanov, and J. A. Hossack, "Dual frequency method for simultaneous translation and real-time imaging of ultrasound contrast agents within large blood vessels," Ultrasound in medicine 63 biology, vol. 35, no. 12, pp. 2021-2030, Dec. 2009.

[149] J. A. Jensen, "A model for the propagation and scattering of ultrasound in tissue," The Journal of the Acoustical Society of America, vol. 89, no. 1, pp. 182-190, Jan. 1991. 
[150] R. J. Zemp, J. Tavakkoli, and R. S. C. Cobbold, "Modeling of nonlinear ultrasound propagation in tissue from array transducers," The Journal of the Acoustical Society of America, vol. 113, no. 1, pp. 139-152, Jan. 2003.

[151] C. C. Church, "The effects of an elastic solid surface layer on the radial pulsations of gas bubbles," The Journal of the Acoustical Society of America, vol. 97, no. 3, pp. 1510-1521, 1995.

[152] K. E. Morgan, J. S. Allen, P. A. Dayton, J. E. Chomas, A. L. Klibaov, and K. W. Ferrara, "Experimental and theoretical evaluation of microbubble behavior: effect of transmitted phase and bubble size," IEEE transactions on ultrasonics, ferroelectrics, and frequency control, vol. 47, no. 6, pp. 1494-1509, 2000.

[153] N. De Jong, A. Bouakaz, and P. Frinking, "Basic acoustic properties of microbubbles," Echocardiography, vol. 19, no. 3, p. 229-240, 2002.

[154] P. Marmottant, S. v. d. Meer, M. Emmer, M. Versluis, N. d. Jong, S. Hilgenfeldt, and D. Lohse, "A model for large amplitude oscillations of coated bubbles accounting for buckling and rupture," The Journal of the Acoustical Society of America, vol. 118, no. 6, pp. 3499-3505, 2005.

[155] A. V. Patil, P. Reynolds, and J. A. Hossack, "A non-linear three-dimensional model for quantifying microbubble dynamics," The Journal of the Acoustical Society of America, vol. 127, no. 2, pp. EL80-86, Feb. 2010.

[156] C. X. Deng, F. Sieling, H. Pan, and J. Cui, "Ultrasound-induced cell membrane porosity," Ultrasound in medicine $\mathscr{G}$ biology, vol. 30, no. 4, pp. 519-526, Apr. 2004.

[157] J. I. Park, Z. Nie, A. Kumachev, and E. Kumacheva, "A microfluidic route to small CO2 microbubbles with narrow size distribution," Soft Matter, vol. 6, no. 3, pp. 630634, Jan. 2010.

[158] J. Holman, Heat Transfer. McGraw-Hill, 2002.

[159] R. G. Bacabac, T. H. Smit, S. C. Cowin, J. J. W. A. Van Loon, F. T. M. Nieuwstadt, R. Heethaar, and J. Klein-Nulend, "Dynamic shear stress in parallel-plate flow chambers," Journal of Biomechanics, vol. 38, no. 1, pp. 159-167, Jan. 2005.

[160] Y. Liu, J. Yan, and M. R. Prausnitz, "Can ultrasound enable efficient intracellular uptake of molecules? a retrospective literature review and analysis," Ultrasound in medicine 85 biology, vol. 38, no. 5, pp. 876-888, May 2012.

[161] J. E. Hall, Guyton and Hall Textbook of Medical Physiology: Enhanced E-book. Elsevier Health Sciences, Jul. 2010.

[162] J. H. Wible, J. K. Wojdyla, G. L. Bales, W. N. McMullen, E. A. Geiser, and D. D. Buss, "Inhaled gases affect the ultrasound contrast produced by albunex $\AA$ in anesthetized dogs," Journal of the American Society of Echocardiography, vol. 9, no. 4, pp. 442-451, Jul. 1996. 
[163] M. S. Tartis, D. E. Kruse, H. Zheng, H. Zhang, A. Kheirolomoom, J. Marik, and K. W. Ferrara, "Dynamic microPET imaging of ultrasound contrast agents and lipid delivery," Journal of controlled release : official journal of the Controlled Release Society, vol. 131, no. 3, pp. 160-166, Nov. 2008.

[164] M. A. Eddings, M. A. Johnson, and B. K. Gale, "Determining the optimal PDMS-PDMS bonding technique for microfluidic devices," Journal of Micromechanics and Microengineering, vol. 18, no. 6, p. 067001, Jun. 2008.

[165] F. Bragheri, P. Minzioni, R. M. Vazquez, N. Bellini, P. Paiè, C. Mondello, R. Ramponi, I. Cristiani, and R. Osellame, "Optofluidic integrated cell sorter fabricated by femtosecond lasers," Lab on a Chip, vol. 12, no. 19, pp. 3779-3784, Aug. 2012.

[166] H. Li, Y. Fan, R. Kodzius, and I. G. Foulds, "Fabrication of polystyrene microfluidic devices using a pulsed CO2 laser system," Microsystem Technologies, vol. 18, no. 3, pp. 373-379, Mar. 2012.

[167] M. E. Frijlink, D. E. Goertz, H. J. Vos, E. Tesselaar, G. Blacquière, A. Gisolf, R. Krams, and A. F. W. van der Steen, "Harmonic intravascular ultrasound imaging with a dual-frequency catheter," Ultrasound in Medicine $\mathcal{E}$ Biology, vol. 32, no. 11, pp. 1649-1654, Nov. 2006.

[168] J. C. Chappell, J. Song, C. W. Burke, A. L. Klibanov, and R. J. Price, "Targeted delivery of nanoparticles bearing fibroblast growth factor-2 by ultrasonic microbubble destruction for therapeutic arteriogenesis," Small, vol. 4, no. 10, pp. 1769-1777, Oct. 2008.

[169] D. F. Gaitan, L. A. Crum, C. C. Church, and R. A. Roy, "Sonoluminescence and bubble dynamics for a single, stable, cavitation bubble," The Journal of the Acoustical Society of America, vol. 91, no. 6, pp. 3166-3183, Jun. 1992.

[170] A. Doinikov and A. Bouakaz, "Review of shell models for contrast agent microbubbles," IEEE Transactions on Ultrasonics, Ferroelectrics and Frequency Control, vol. 58, no. 5, pp. 981-993, 2011.

[171] T. G. Leighton, "Cavitation inception and fluid dynamics," in The Acoustic Bubble. Elsevier, 1994, pp. 100-106.

[172] P. S. Epstein and M. S. Plesset, "On the stability of gas bubbles in liquid-gas solutions," The Journal of Chemical Physics, vol. 18, no. 11, p. 1505, 1950.

[173] K. Sarkar, A. Katiyar, and P. Jain, "Growth and dissolution of an encapsulated contrast microbubble: Effects of encapsulation permeability," Ultrasound in Medicine ES Biology, vol. 35, no. 8, pp. 1385-1396, Aug. 2009.

[174] F. Conversano, R. Franchini, A. Lay-Ekuakille, and S. Casciaro, "In vitro evaluation and theoretical modeling of the dissolution behavior of a microbubble contrast agent for ultrasound imaging," IEEE Sensors Journal, vol. 12, no. 3, pp. 496-503, Mar. 2012. 
[175] R. T. Ferrell and D. M. Himmelblau, "Diffusion coefficients of nitrogen and oxygen in water," Journal of Chemical \&f Engineering Data, vol. 12, no. 1, pp. 111-115, Jan. 1967.

[176] E. Wilhelm, R. Battino, and R. J. Wilcock, "Low-pressure solubility of gases in liquid water," Chemical Reviews, vol. 77, no. 2, pp. 219-262, Apr. 1977.

[177] W. Yan, G.-Y. Zhao, G.-J. Chen, and T.-M. Guo, "Interfacial tension of (methane + nitrogen) + water and (carbon dioxide + nitrogen) + water systems," Journal of Chemical \& Engineering Data, vol. 46, no. 6, pp. 1544-1548, Nov. 2001.

[178] D. R. Absolom, C. J. Van Oss, W. Zingg, and A. W. Neumann, "Determination of surface tensions of proteins II. surface tension of serum albumin, altered at the protein-air interface," Biochimica et Biophysica Acta (BBA) - Protein Structure, vol. 670, no. 1, pp. 74-78, Aug. 1981.

[179] P. B. Duncan and D. Needham, "Test of the Epstein-Plesset model for gas microparticle dissolution in aqueous media: effect of surface tension and gas undersaturation in solution," Langmuir, vol. 20, no. 7, pp. 2567-2578, Mar. 2004.

[180] M. Yamada and M. Seki, "Hydrodynamic filtration for on-chip particle concentration and classification utilizing microfluidics," Lab on a Chip, vol. 5, no. 11, pp. 12331239, Oct. 2005.

[181] A. Kobayashi, M. Yamada, and M. Seki, "Bubble-based continuous separation system in microfluidic device," in 14th International Conference on Miniaturized Systems for Chemistry and Life Sciences, Groningen, The Netherlands, Oct. 2010, pp. 1364-1366.

[182] K. R. Nightingale, M. L. Palmeri, R. W. Nightingale, and G. E. Trahey, "On the feasibility of remote palpation using acoustic radiation force," The Journal of the Acoustical Society of America, vol. 110, no. 1, pp. 625-634, Jul. 2001.

[183] A. P. Sarvazyan, O. V. Rudenko, S. D. Swanson, J. B. Fowlkes, and S. Y. Emelianov, "Shear wave elasticity imaging: a new ultrasonic technology of medical diagnostics," Ultrasound in Medicine \& Biology, vol. 24, no. 9, pp. 1419-1435, Nov. 1998.

[184] S. Chen, M. Fatemi, and J. F. Greenleaf, "Quantifying elasticity and viscosity from measurement of shear wave speed dispersion," The Journal of the Acoustical Society of America, vol. 115, no. 6, pp. 2781-2785, Jun. 2004.

[185] J. Bercoff, M. Tanter, and M. Fink, "Supersonic shear imaging: a new technique for soft tissue elasticity mapping," IEEE Transactions on Ultrasonics, Ferroelectrics, and Frequency Control, vol. 51, no. 4, pp. 396-409, Apr. 2004.

[186] S. A. McAleavey, M. Menon, and J. Orszulak, "Shear-modulus estimation by application of spatially-modulated impulsive acoustic radiation force," Ultrasonic Imaging, vol. 29, no. 2, pp. 87-104, Apr. 2007. 
[187] M. Fink and M. Tanter, "Multiwave imaging and super resolution," Physics Today, vol. 63 , no. 2, p. 28, 2010.

[188] E. Bavu, J.-L. Gennisson, V. Mallet, B.-F. Osmanski, M. Couade, J. Bercoff, M. Fink, P. Sogni, A. Vallet-Pichard, and B. Nalpas, "406 supersonic shear imaging is a new potent morphological non-invasive technique to assess liver fibrosis. part i: Technical feasibility," Journal of Hepatology, vol. 52, pp. S166-S166, Apr. 2010.

[189] F. W. Mauldin, M. A. Haider, E. G. Loboa, R. H. Behler, L. E. Euliss, T. W. Pfeiler, and C. M. Gallippi, "Monitored steady-state excitation and recovery (MSSER) radiation force imaging using viscoelastic models," IEEE Transactions on Ultrasonics, Ferroelectrics, and Frequency Control, vol. 55, no. 7, pp. 1597-1610, Jul. 2008.

[190] B. J. Fahey, R. C. Nelson, D. P. Bradway, S. J. Hsu, D. M. Dumont, and G. E. Trahey, "In vivo visualization of abdominal malignancies with acoustic radiation force elastography," Physics in Medicine and Biology, vol. 53, no. 1, pp. 279-293, Jan. 2008 .

[191] B. Fahey, K. Nightingale, R. Nelson, M. Palmeri, and G. Trahey, "Acoustic radiation force impulse imaging of the abdomen: demonstration of feasibility and utility," Ultrasound in Medicine E Biology, vol. 31, no. 9, pp. 1185-1198, Sep. 2005.

[192] S. J. Hsu, R. R. Bouchard, D. M. Dumont, P. D. Wolf, and G. E. Trahey, "In vivo assessment of myocardial stiffness with acoustic radiation force impulse imaging," Ultrasound in Medicine \& Biology, vol. 33, no. 11, pp. 1706-1719, Nov. 2007.

[193] M. L. Palmeri, M. H. Wang, J. J. Dahl, K. D. Frinkley, and K. R. Nightingale, "Quantifying hepatic shear modulus in vivo using acoustic radiation force," Ultrasound in Medicine \& Biology, vol. 34, no. 4, pp. 546-558, Apr. 2008.

[194] A. Sharma, M. Soo, G. Trahey, and K. Nightingale, "Acoustic radiation force impulse imaging of in vivo breast masses," in 2004 IEEE Ultrasonics Symposium, vol. 1, 2004, pp. 728-731 Vol.1.

[195] L. Zhai, J. Madden, W.-C. Foo, M. L. Palmeri, V. Mouraviev, T. J. Polascik, and K. R. Nightingale, "Acoustic radiation force impulse imaging of human prostates ex vivo," Ultrasound in Medicine $\&$ Biology, vol. 36, no. 4, pp. 576-588, Apr. 2010.

[196] C. Hartley, "Characteristics of acoustic streaming created and measured by pulsed doppler ultrasound," IEEE Transactions on Ultrasonics, Ferroelectrics and Frequency Control, vol. 44, no. 6, pp. 1278-1285, Nov. 1997.

[197] F. W. Mauldin, F. Viola, T. C. Hamer, E. M. Ahmed, S. B. Crawford, D. M. Haverstick, M. B. Lawrence, and W. F. Walker, "Adaptive force sonorheometry for assessment of whole blood coagulation," Clinica Chimica Acta; International Journal of Clinical Chemistry, vol. 411, no. 9-10, pp. 638-644, May 2010. 
[198] S. A. McAleavey, K. R. Nightingale, and G. E. Trahey, "Estimates of echo correlation and measurement bias in acoustic radiation force impulse imaging," IEEE Transactions on Ultrasonics, Ferroelectrics, and Frequency Control, vol. 50, no. 6, pp. 631-641, Jun. 2003.

[199] M. L. Palmeri, S. A. McAleavey, G. E. Trahey, and K. R. Nightingale, "Ultrasonic tracking of acoustic radiation force-induced displacements in homogeneous media," IEEE Transactions on Ultrasonics, Ferroelectrics, and Frequency Control, vol. 53, no. 7, pp. 1300-1313, Jul. 2006.

[200] K. Nightingale, M. Soo, R. Nightingale, R. Bentley, D. Stutz, M. Palmeri, J. Dahl, and G. Trahey, "Acoustic radiation force impulse imaging: remote palpation of the mechanical properties of tissue," in 2002 IEEE Ultrasonics Symposium, 2002. Proceedings, vol. 2, Oct. 2002, pp. 1821-1830 vol.2.

[201] F. W. Mauldin, F. Viola, and W. F. Walker, "Reduction of echo decorrelation via complex principal component filtering," Ultrasound in Medicine 6 Biology, vol. 35, no. 8, pp. 1325-1343, Aug. 2009.

[202] ——, "Complex principal components for robust motion estimation," IEEE Transactions on Ultrasonics, Ferroelectrics, and Frequency Control, vol. 57, no. 11, pp. 2437-2449, Nov. 2010.

[203] W. Walker and G. Trahey, "A fundamental limit on delay estimation using partially correlated speckle signals," IEEE Transactions on Ultrasonics, Ferroelectrics and Frequency Control, vol. 42, no. 2, pp. 301-308, Mar. 1995.

[204] M. L. Palmeri, A. C. Sharma, R. R. Bouchard, R. W. Nightingale, and K. R. Nightingale, "A finite-element method model of soft tissue response to impulsive acoustic radiation force," IEEE Transactions on Ultrasonics, Ferroelectrics, and Frequency Control, vol. 52, no. 10, pp. 1699-1712, Oct. 2005.

[205] M. L. Palmeri, S. A. McAleavey, K. L. Fong, G. E. Trahey, and K. R. Nightingale, "Dynamic mechanical response of elastic spherical inclusions to impulsive acoustic radiation force excitation," IEEE Transactions on Ultrasonics, Ferroelectrics, and Frequency Control, vol. 53, no. 11, pp. 2065-2079, Nov. 2006.

[206] C. M. Gallippi, K. R. Nightingale, and G. E. Trahey, "BSS-based filtering of physiological and ARFI-induced tissue and blood motion," Ultrasound in Medicine \& Biology, vol. 29, no. 11, pp. 1583-1592, Nov. 2003.

[207] R. H. Behler, T. C. Nichols, H. Zhu, E. P. Merricks, and C. M. Gallippi, "ARFI imaging for noninvasive material characterization of atherosclerosis. part II: toward in vivo characterization," Ultrasound in Medicine 6 Biology, vol. 35, no. 2, pp. 278295, Feb. 2009.

[208] M. W. Urban, S. Chen, and J. Greenleaf, "Harmonic motion detection in a vibrating scattering medium," IEEE Transactions on Ultrasonics, Ferroelectrics, and Frequency Control, vol. 55, no. 9, pp. 1956-1974, Sep. 2008. 
[209] J. A. Jensen and N. B. Svendsen, "Calculation of pressure fields from arbitrarily shaped, apodized, and excited ultrasound transducers," IEEE Transactions on Ultrasonics, Ferroelectrics, and Frequency Control, vol. 39, no. 2, pp. 262-267, 1992.

[210] G. R. Torr, "The acoustic radiation force," American Journal of Physics, vol. 52, no. 5, pp. 402-408, May 1984.

[211] Y. Saad and M. H. Schultz, "GMRES: a generalized minimal residual algorithm for solving nonsymmetric linear systems," SIAM Journal on Scientific and Statistical Computing, vol. 7, no. 3, p. 856, 1986.

[212] W. Hackbusch, Multi-grid methods and applications. Springer, 1985.

[213] T. Loupas, J. Powers, and R. Gill, "An axial velocity estimator for ultrasound blood flow imaging, based on a full evaluation of the doppler equation by means of a twodimensional autocorrelation approach," IEEE Transactions on Ultrasonics, Ferroelectrics and Frequency Control, vol. 42, no. 4, pp. 672-688, Jul. 1995.

[214] K. Nightingale, M. Palmeri, and G. Trahey, "Analysis of contrast in images generated with transient acoustic radiation force," Ultrasound in Medicine 65 Biology, vol. 32, no. 1, pp. 61-72, Jan. 2006.

[215] F. A. Duck, Physical properties of tissue: a comprehensive reference book. Academic Press, 1990.

[216] M. Lediju, M. Pihl, S. Hsu, J. Dahl, C. Gallippi, and G. Trahey, "A motion-based approach to abdominal clutter reduction," IEEE Transactions on Ultrasonics, Ferroelectrics and Frequency Control, vol. 56, no. 11, pp. 2437-2449, Nov. 2009.

[217] J. Kilroy, A. Klibanov, B. Wamhoff, and J. Hossack, "Intravascular ultrasound catheter to enhance microbubble-based drug delivery via acoustic radiation force," IEEE Transactions on Ultrasonics, Ferroelectrics and Frequency Control, vol. 59, no. 10, pp. 2156-2166, 2012.

[218] K. Kooiman, M. R. Böhmer, M. Emmer, H. J. Vos, C. Chlon, W. T. Shi, C. S. Hall, S. H. de Winter, K. Schroën, M. Versluis, N. de Jong, and A. van Wamel, "Oil-filled polymer microcapsules for ultrasound-mediated delivery of lipophilic drugs," Journal of Controlled Release, vol. 133, no. 2, pp. 109-118, Jan. 2009.

[219] A. L. Klibanov, T. I. Shevchenko, B. I. Raju, R. Seip, and C. T. Chin, "Ultrasoundtriggered release of materials entrapped in microbubble-liposome constructs: a tool for targeted drug delivery," Journal of controlled release: official journal of the Controlled Release Society, vol. 148, no. 1, pp. 13-17, Nov. 2010.

[220] J. Escoffre, C. Mannaris, B. Geers, A. Novell, I. Lentacker, M. Averkiou, and A. Bouakaz, "Doxorubicin liposome-loaded microbubbles for contrast imaging and ultrasound-triggered drug delivery," IEEE Transactions on Ultrasonics, Ferroelectrics and Frequency Control, vol. 60, no. 1, pp. -, 2013. 
[221] L. Rossi, S. Serafini, F. Pierigé, A. Antonelli, A. Cerasi, A. Fraternale, L. Chiarantini, and M. Magnani, "Erythrocyte-based drug delivery," Expert Opinion on Drug Delivery, vol. 2, no. 2, pp. 311-322, Mar. 2005.

[222] F. Pierige, S. Serafini, L. Rossi, and M. Magnani, "Cell-based drug delivery," Advanced Drug Delivery Reviews, vol. 60, no. 2, pp. 286-295, Jan. 2008.

[223] P. D. Patel, N. Dand, R. S. Hirlekar, and V. J. Kadam, "Drug loaded erythrocytes: as novel drug delivery system," Current Pharmaceutical Design, vol. 14, no. 1, pp. 63-70, 2008.

[224] V. R. Muzykantov, "Drug delivery by red blood cells: vascular carriers designed by mother nature," Expert Opinion on Drug Delivery, vol. 7, no. 4, pp. 403-427, Apr. 2010.

[225] B. E. Bax, M. D. Bain, P. J. Talbot, E. J. Parker-Williams, and R. A. Chalmers, "Survival of human carrier erythrocytes in vivo," Clinical Science (London, England: 1979), vol. 96, no. 2, pp. 171-178, Feb. 1999.

[226] B. E. Bax, M. D. Bain, L. D. Fairbanks, A. D. B. Webster, P. W. Ind, M. S. Hershfield, and R. A. Chalmers, "A 9-yr evaluation of carrier erythrocyte encapsulated adenosine deaminase (ADA) therapy in a patient with adult-type ADA deficiency," European Journal of Haematology, vol. 79, no. 4, pp. 338-348, Oct. 2007.

[227] P. Seeman, "Transient holes in the erythrocyte membrane during hypotonic hemolysis and stable holes in the membrane after lysis by saponin and lysolecithin," The Journal of Cell Biology, vol. 32, no. 1, pp. 55-70, Jan. 1967.

[228] M. R. Lieber and T. L. Steck, "Dynamics of the holes in human erythrocyte membrane ghosts," The Journal of biological chemistry, vol. 257, no. 19, pp. 11660 11666 , Oct. 1982.

[229] V. Lew, A. Hockaday, C. Freeman, and R. Bookchin, "Mechanism of spontaneous inside-out vesiculation of red-cell membranes," Journal of Cell Biology, vol. 106, no. 6, pp. 1893-1901, Jun. 1988, WOS:A1988P139500010.

[230] M. Magnani, Erythrocyte engineering for drug delivery and targeting. Georgetown Tex.;New York: Landes Bioscience/Eurekah.com;;Kluwer Academic/Plenum Publishers, 2003.

[231] K. K. Shung, R. Sigelmann, and J. Reid, "Scattering of ultrasound by blood," IEEE Transactions on Biomedical Engineering, vol. BME-23, no. 6, pp. 460-467, Nov. 1976.

[232] G. Schwoch and H. Passow, "Preparation and properties of human erythrocyte ghosts," Molecular and Cellular Biochemistry, vol. 2, pp. 197-218, Dec. 1973. 
[233] M. P. Krafft and J. G. Riess, "Chemistry, physical chemistry, and uses of molecular Fluorocarbon-Hydrocarbon diblocks, triblocks, and related compounds - unique "Apolar" components for self-assembled colloid and interface engineering," Chemical Reviews, vol. 109, no. 5, pp. 1714-1792, May 2009.

[234] T. L. Steck and J. A. Kant, "[16] preparation of impermeable ghosts and insideout vesicles from human erythrocyte membranes," in Methods in Enzymology, L. P. Sidney Fleischer, Ed. Academic Press, 1974, vol. Volume 31, pp. 172-180.

[235] T. Giesecke and K. Hynynen, "Ultrasound-mediated cavitation thresholds of liquid perfluorocarbon droplets in vitro," Ultrasound in Medicine \& Biology, vol. 29, no. 9, pp. 1359-1365, Sep. 2003.

[236] P. S. Sheeran, V. P. Wong, S. Luois, R. J. McFarland, W. D. Ross, S. Feingold, T. O. Matsunaga, and P. A. Dayton, "Decafluorobutane as a phase-change contrast agent for low-energy extravascular ultrasonic imaging," Ultrasound in Medicine \& Biology, vol. 37, no. 9, pp. 1518-1530, Sep. 2011.

[237] K. Soetanto and M. Chan, "Fundamental studies on contrast images from differentsized microbubbles: analytical and experimental studies," Ultrasound in Medicine $\&$ Biology, vol. 26, no. 1, pp. 81-91, Jan. 2000.

[238] G. Liessmann, W. Schmidt, and S. Reiffarth, "Recommended thermophysical data," in Data compilation of the Saechsische Olefinwerke, Boehlen, Germany, 1995, p. 96.

[239] W. Hayduk and H. Laudie, "Prediction of diffusion coefficients for nonelectrolytes in dilute aqueous solutions," AIChE Journal, vol. 20, no. 3, pp. 611-615, May 1974.

[240] A. Kabalnov, K. Makarov, O. Shcherbakova, and A. Nesmeyanov, "Solubility of fluorocarbons in water as a key parameter determining fluorocarbon emulsion stability," Journal of Fluorine Chemistry, vol. 50, no. 3, pp. 271-284, Dec. 1990.

[241] P. J. Ghyzel, "Stabilized coating dispersions for porous inkjet recording media," U.S. Patent US8 367756 B2, Feb., 2013, U.S. Classification 524/377, 524/493, 524/366, 524/52, 524/462, 524/847, 524/47; International Classification C08K5/053, C08K5/02, C08K3/36; Cooperative Classification C08L5/00, B41M5/52, C09D129/04, B41M5/5218, C08K3/36, C08K5/06, B41M5/5227.

[242] G. M. Ihler, R. H. Glew, and F. W. Schnure, "Enzyme loading of erythrocytes," Proceedings of the National Academy of Sciences, vol. 70, no. 9, pp. 2663-2666, Sep. 1973. 